\title{
Evolution of caudal translational repression in higher insects
}

\author{
Dissertation submitted in partial fulfilment of the requirements for the degree of \\ "Doctor rerum naturalium" \\ of the Georg-August-University Göttingen
}

by

Claudia Jasmin Rödel

Bremerhaven,

Germany

Göttingen, 2010 
First Referee: $\quad$ Prof. Dr. Ernst Wimmer (Department of Developmental Biology, Georg-August-University Göttingen, Germany)

Second Referee: Prof. Dr. Gregor Bucher (Department of Developmental Biology, Georg-August-University Göttingen, Germany)

Date of submission: 07. December 2010 
The experimental part of this thesis was performed at the

Department of Developmental Biology

Institute of Molecular Biology and Biotechnology (IMBB)

Foundation for Research and Technology - Hellas (FoRTH)

Heraklion, Crete, Greece

under the supervision of Dr. Michalis Averof. 
Herewith I declare that I prepared the Dissertation

"Evolution of caudal translational repression in higher insects"

on my own and with no other source and aids than quoted.

Göttingen, 07. December 2010

Claudia Rödel 
Index

Index I

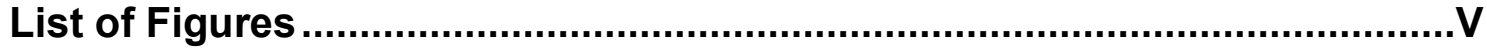

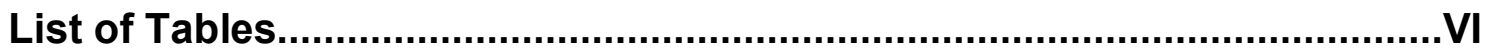

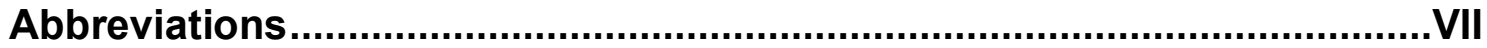

1 Abstract

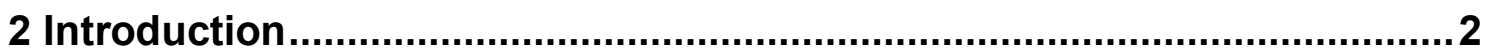

2.1 AP axis determination and polarity during insect development..........2

2.1.1 AP axis determination in Drosophila melanogaster ........................ 3

2.1.2 AP axis determination in other insects ...................................... 6

2.2 The anterior patterning factor Bicoid ................................................ 10

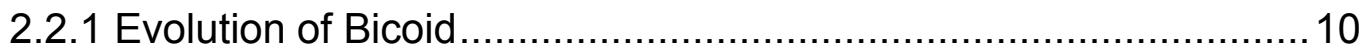

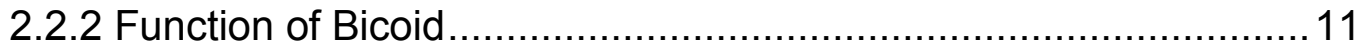

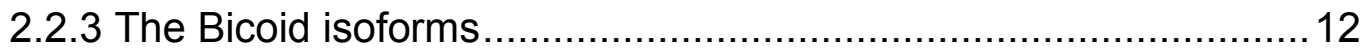

2.3 Mechanism of $\mathrm{cad}$ translational regulation in Drosophila...................14

2.4 miRNA function during early Drosophila development ......................16

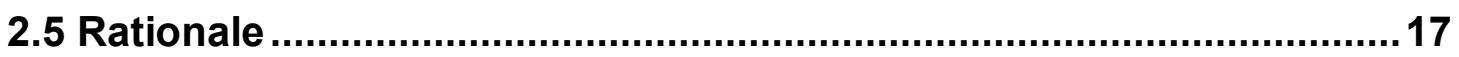

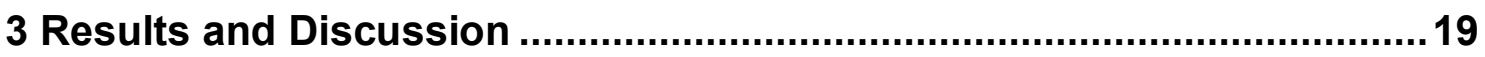

3.1 Mapping of BCD-binding RNA-elements in vitro .................................19

3.1.1 Establishment of RNA-binding assay using recombinant BCD

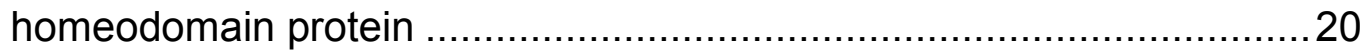

3.1.2 Analysis of recombinant BCD homeodomain binding to RNA .......22

3.1.3 Characterization of the BCD binding element BRE_257-319 in the Drosophila cad 3'UTR

3.1.4 Identification of a Haematopota cad 3'UTR element that is bound

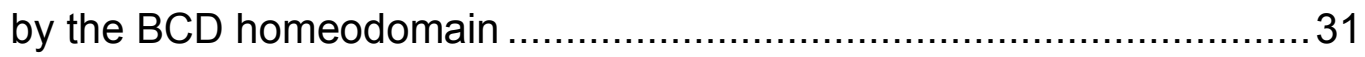

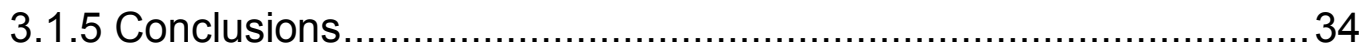

3.2 Mapping Bicoid RNA-binding elements in vivo..................................36 
3.2.1 The making of a fluorescent protein reporter assay in early

Drosophila embryogenesis 36

3.2.1.1 Strategy 1: Translational reporter assay using a single $\varphi \mathrm{C} 31$ integrase recombination site 39

3.2.1.2 Strategy 2: Translational reporter assay using the $\varphi \mathrm{C} 31$-integrase mediated cassette exchange (RMCE)

3.2.1.3 Strategy 2b: A translational reporter assay using RMCE in conjunction with BCD protein expressed using the UAS/GAL4 induction system.

3.2.1.4 Strategy 3: Translational reporter assay using a d2EGFP-CAD fusion 46

3.2.1.5 Conclusions

3.2.2 Identification of cad 3'UTR homologues that are translationally regulated by $B C D$

3.2.2.1 The BRE_257-319 fragment of the cad 3'UTR is mediating translational repression in the presence of BCD

3.2.2.2 The Haematopota cad 3'UTR mediates translational repression in the presence of $B C D$

3.2.2.3 The Tribolium cad 3'UTR fails to mediate BCD-dependent translational repression of the dnGFP sensor.

3.2.2.4 Conclusions.

3.3 Testing the role of different Bicoid isoforms during Drosophila early embryogenesis

3.3.1 $h b$ transcriptional activation and CAD translational repression by different BCD isoforms

3.3.2 Translational repression of sensor 38F-Dm3' by different BCD isoforms.

3.3.3 Conclusions

3.4 Hints for alternative mechanisms of BCD-mediated translational repression

3.4.1 Mutations in the miR-308 target site abolish BCD-mediated translational repression

3.4.2 Translational repression of 38F-BRE_257-319 and 38F-H2 by BCD isoform $\mathrm{F}$ 


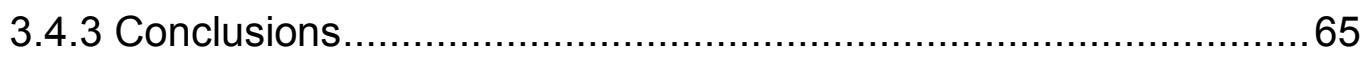

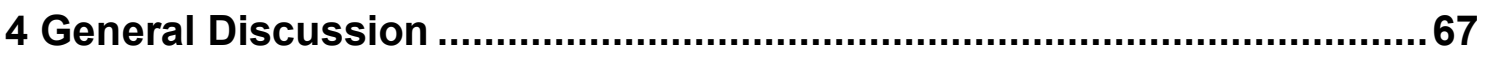

4.1 Insights into translational control of cad in Drosophila .....................67

4.2 Alternative mechanisms of translational control of cad mRNA.........71

4.2.1 Evidence for d4EHP-independent cad translational repression ....71

4.2.2 The putative role of miR-308 in BCD-dependent cad translational

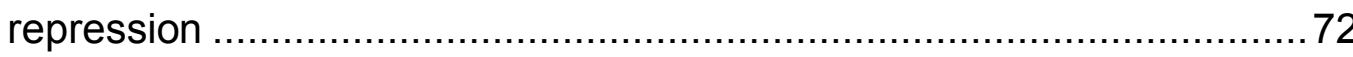

4.3 Implications for the evolution of $c a d$ translational regulation ............74

4.4 Summary and future directions .........................................................78

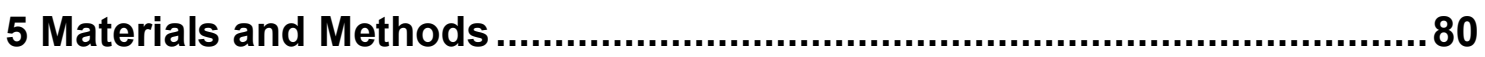

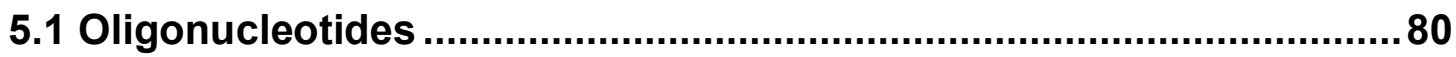

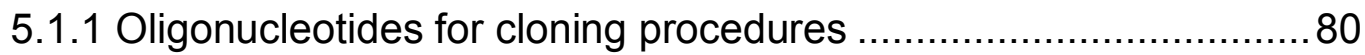

5.1.2 Oligonucleotides for template generation of in vitro synthesized

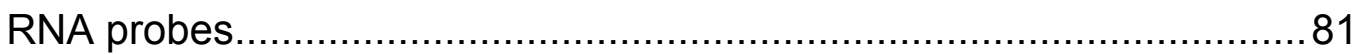

5.1.3 Oligonucleotides for generation of ds DNA probe ....................... 81

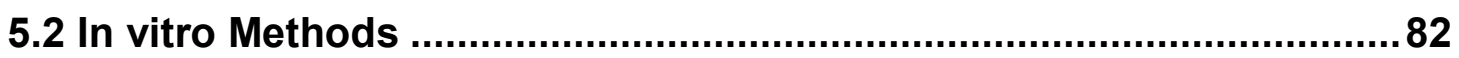

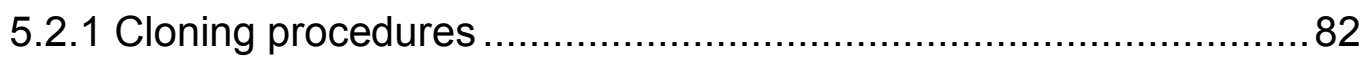

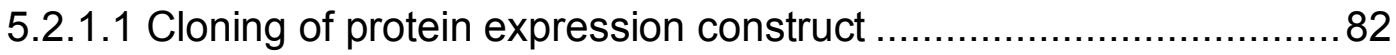

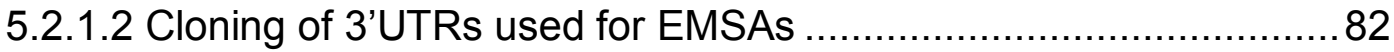

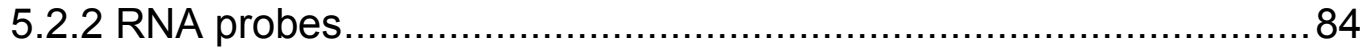

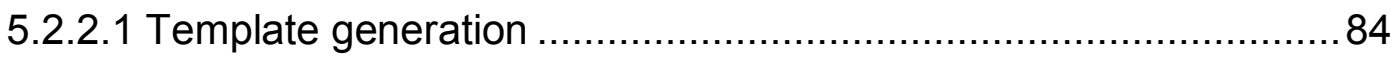

5.2.2.2 In vitro transcription and radioisotope-labeling of RNA probes...... 85

5.2.3 Radioisotope-labeling of dsDNA oligonucelotides........................ 86

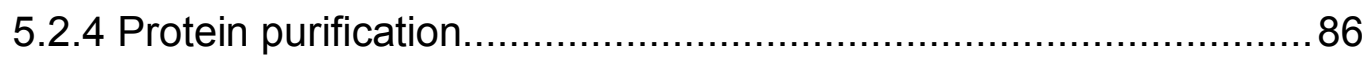

5.2.4.1 Purification of HisMBP-HD fusion protein ..................................... 86

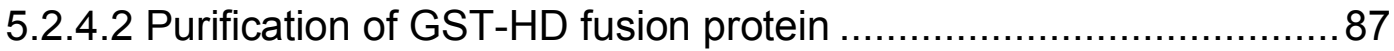

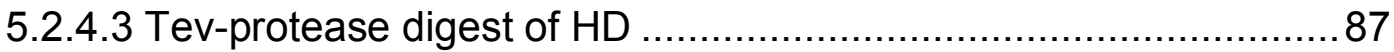

5.2.5 Electrophoretic Mobility Shift Assays (EMSAs) .......................... 88

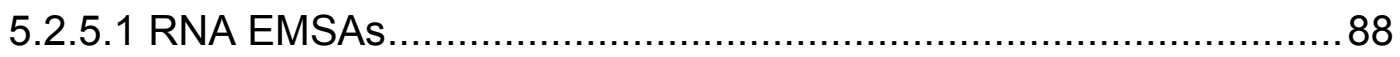

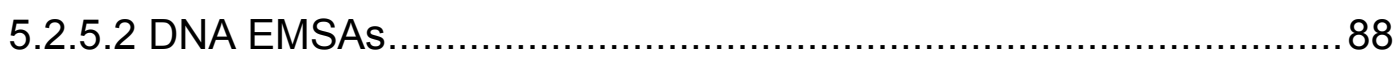

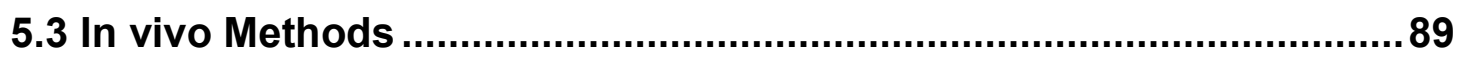

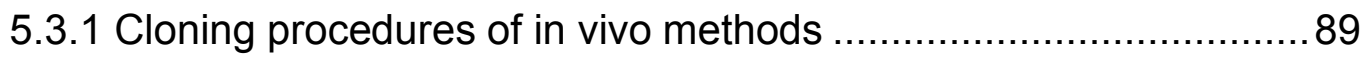

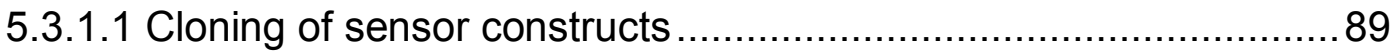




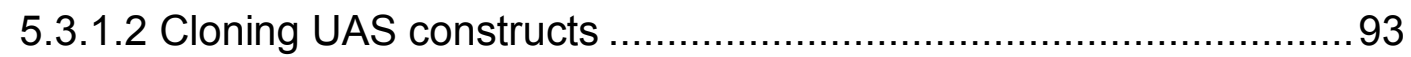

5.3.2 Drosophila melanogaster handling and husbandry .................... 94

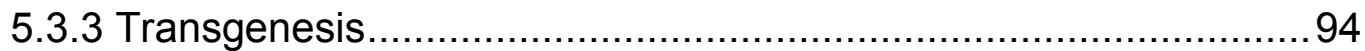

5.3.3.1 $\varphi$ C31 integrase mediated recombination system using a single attP landing site (strategy 1)

5.3.3.2 Recombinase-mediated exchange cassette (RMCE) using $\varphi \mathrm{C} 31$

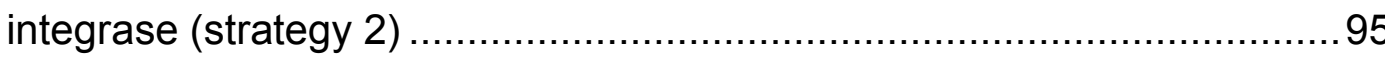

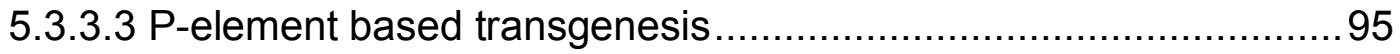

5.3.4 Drosophila husbandry for expression of UAS-BCD isoforms during early embryogenesis .

5.3.5 Drosophila husbandry for expression of UAS-BCD and sensor constructs during early embryogenesis ...................................... 97

5.3.6 Immunohistochemistry ................................................ 100

5.3.6.1 Antibody stainings .................................................... 100

5.3.6.2 In situ hybridization .................................................... 101

5.3.7 Microscopy and Imaging ................................................. 102

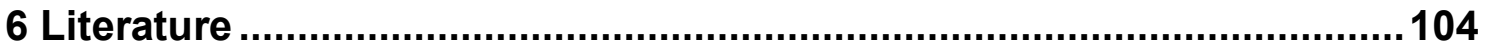

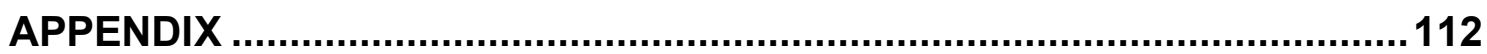

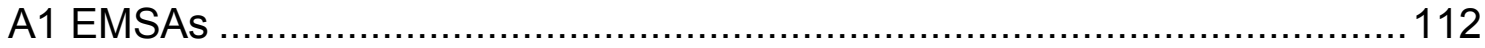

A2 Alignment of cad 3'UTR homologues ............................................... 116

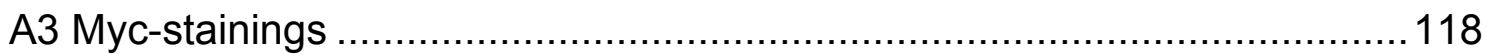

A4 Secondary structure predictions and RNAforester alignments ............... 119

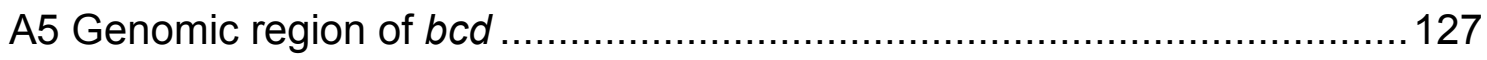

A6 Measurements of in vivo translational reporter assay ......................... 130

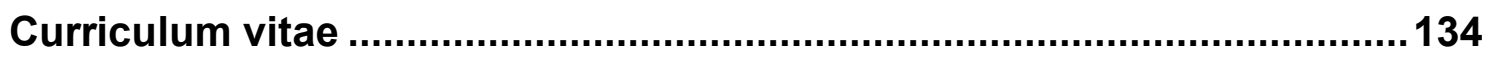

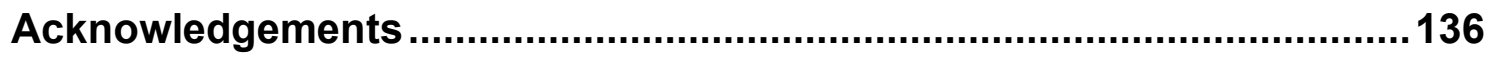




\section{List of Figures}

Fig. 2.1: AP axis pattering and segmentation gene cascade in Drosophila melanogaster.

Fig. 2.2: Long-germband development vs. short-germ development and the evolution of Hox3-like genes in the lineage of Tribolium and Drosophila.

Fig. 2.3: Schematic overview of putative evolution of bcd and zen in taxa of diptera...11

Fig. 2.4: The protein domains of $B C D$ isoform $G$.

Fig. 2.5: Schematic overview of the bcd transcripts produced by differential splicing of four major exons. 14

Fig. 2.6: Model of cad translational repression, proposed by Cho et al. (2005). 15

Fig. 3.1: Drosophila cad 3' UTR and RNA fragments previously described as Bicoid

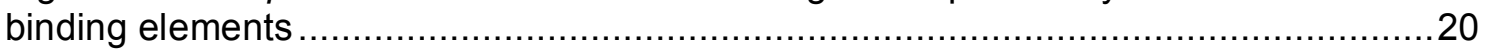

Fig. 3.2: DNA- and RNA-binding assay of BCD homeodomain............................24

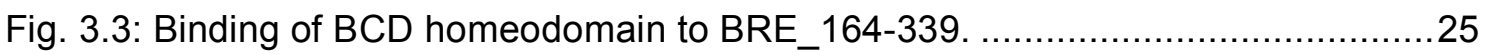

Fig. 3.4: Sequence alignment of cad 3'UTRs from different Drosophila species..........28

Fig. 3.5: Binding of BCD homeodomain to BRE_257-319 …...................................29

Fig. 3.6: Specificity of BCD homeodomain binding to BRE_257-319 ...................... 30

Fig. 3.7: Comparision of secondary structures of Hp3' and BRE_257-319. ................32

Fig. 3.8: Binding of the BCD homeodomain to Haematopota 3'UTR fragments H1, H2

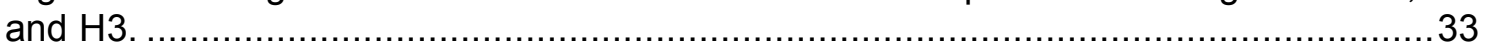

Fig. 3.9: Schematic overview of translational reporter assays. ..................................38

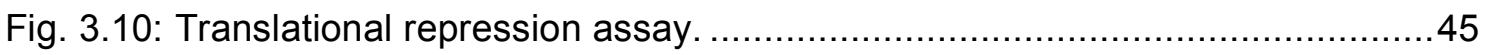

Fig. 3.11: Translational repression of a reporter carrying a d2GFP-CAD fusion and the

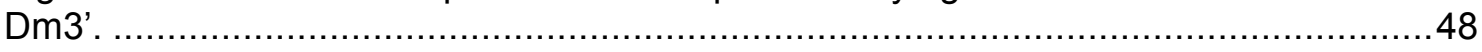

Fig. 3.12: Translational repression assay on BRE_257-319, Haematopota and

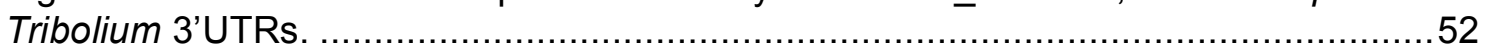

Fig. 3.13: $h b$ expression in the presence of differen BCD isoforms. ..........................56

Fig. 3.14: cad translational repression in the presence of different BCD isoforms. ......57

Fig. 3.15: Translational repression effect of different BCD isoforms on the 38F-Dm3'

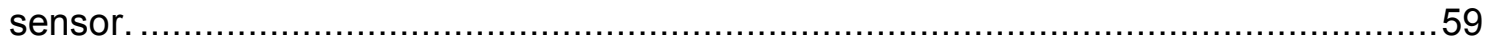

Fig. 3.16: Predicted secondary structures of BRE_257-319 and $\mathrm{H} 2$. .......................62

Fig. 3.17: Translational reporter assay for repression of the mutated cad 3'UTR in sensor 38F-Dm3'mut by different BCD isoforms.

Fig. 3.18: Translational reporter assay for repression of BRE_257-319 and H2 by BCD isoforms $G$ and $F$.

Fig. 4.1: Phylogeny of insect species (after Savard et al., 2006) discussed with features of cad translational repression mapped on. 


\section{List of Tables}

Tab. 3.1: Drosophila sensor lines generated by $\varphi$ C31-integrase mediated insertion into the pJBattPM44 locus on the second chromosome. 40

Tab. 3.2: Drosophila sensor lines generated by $\varphi$ C31-integrase-mediated RMCE. .....42

Tab. 4.1: Presence of d4EHP-binding domain in different BCD isoforms and their ability to mediate translational repression of different sensors. 


\section{Abbreviations}

우

aa

AP

as

ATP

BCIP

bp

cap

cDNA

DIG

Dm

ds

DTT

EDTA

FA

$\mathrm{h}$

HD

HEPES

$\mathrm{Hp}$

$\mathrm{kb}$

LB

LEW

$\min$

miRNA

mRNA

NBT

nt

OD

ORF

PCR

PEM

rpm

RT female

male

amino acid

Alkaline Phosphatase

antisense

adenosine triphosphate

5-Bromo-4-chloro-3-indolyl phosphate

base pair

7-methylguanosine cap structure

complementary DNA

Digoxigenin-11-2'-deoxyuridin-5'-triphosphate

Drosophila melanogaster

double stranded

1,4-Dithiothreit

Ethylediamintetraacetate

formaldehyde

hours

homeodomain

4-(2-Hydroxyethyl)-1-piperazin

Haematopota pluvialis

Kilobase

Luria-Bertani (medium)

Lysis-Elution-Wash-buffer

minutes

micro RNA

messenger RNA

Nitro blue tetrazolium chloride

nucleotide

Optical density

open reading frame

Polymerase chain reaction

Pipes-EGTA-MgCl -buffer

rounds per minute

Room temperature 


$\begin{array}{ll}\text { sec } & \text { seconds } \\ \text { SSC } & \text { Standar Sline Citrate Buffer } \\ \text { TBE } & \text { Tris-Borate-EDAT-Electrophoressis buffer } \\ \text { TC } & \text { Tribolium castaneum } \\ \text { tRNA } & \text { transfer RNA } \\ \text { UTR } & \text { untranslated region }\end{array}$

\section{Abbreviations of amino acids}

$\begin{array}{ll}\text { A } & \text { Alanin } \\ \text { C } & \text { Cystein } \\ \text { E } & \text { Aspartate } \\ \text { F } & \text { Glutamate } \\ \text { G } & \text { Phenylalanin } \\ \text { H } & \text { Glycine } \\ \text { I } & \text { Histidine } \\ \text { K } & \text { Isoleucine } \\ \text { L } & \text { Lysine } \\ \text { M } & \text { Leucine } \\ \text { N } & \text { Methionine } \\ \text { P } & \text { Asparagine } \\ \text { Q } & \text { Proline } \\ \text { R } & \text { Glutamine } \\ \text { S } & \text { Arginine } \\ \text { T } & \text { Serine } \\ \text { V } & \text { Threonine } \\ \text { W } & \text { Valine } \\ \text { Y } & \text { Tryptophane } \\ & \text { Tyrosine }\end{array}$

\section{Abbrevations of nucleotides}
A
Adenine
C
Cytosine
G
Guanine
T
Thymine
U
Uridine 


\section{Abstract}

caudall $C d x$ genes are homeobox genes involved in posterior patterning of the embryo in a wide range of bilaterian species, including arthropods, nematodes and vertebrates. In the fruit fly Drosophila melanogaster the homeobox gene bicoid (bcd), an anterior patterning factor that orchestrates anterior patterning, prevents translation of the uniformly distributed cad mRNA. The mechanism through which BCD mediates this repression has been proposed as a new paradigm for translational control: BCD binds directly through its homeodomain to the cad 3' UTR and simultaneously interacts with the cap-binding protein d4EHP. Thus the cad mRNA remains translationally repressed.

The anterior patterning factor bcd is an evolutionary novelty present only in higher dipterans, therefore other insect species must follow different strategies to restrict cad expression to the posterior. In the beetle Tribolium castaneum, the homeodomain protein Tc'ZEN-2 and the KH-domain protein Tc'MEX-3 restrict Tc'caudal (Tc'cad) to the posterior part of the embryo. Nevertheless, when expressed in Drosophila embryos, BCD translationally represses $T c^{\prime}$ cad mRNA. The region to which BCD binds has been speculated to be in the Tc'cad 3'UTR and raised the question whether BCD may recognize regulatory element(s) that are conserved between Drosophila and Tribolium.

By establishing an in vivo sensor for BCD-mediated translational repression I was able to identify small regions in the cad 3'UTR of Drosophila and the horsefly Haematopota pluvialis that mediate BCD-dependent translational repression. These elements show similarities in their predicted secondary structures, which could be the basis for a conserved BCD-binding element. Using electrophoretic mobility shift assays I could show direct binding of the BCD homeodomain to these 3'UTR regions. Surprisingly, the 3'UTR of the Tc'cad homologue does not seem to contain BCDbinding elements.

The BCD-binding region of the Dm'cad 3'UTR co-localizes with a target site of the microRNA miR-308 and mutations in this region abolish miRNA-binding and BCDmediated translational repression. Furthermore, BCD isoforms that lack the d4EHPbinding domain are able to mediate translational repression of sensors carrying BCDbinding regions. Taken together, these findings suggest that alternative mechanism(s) for the translational repression of cad mRNA are likely to exist in Drosophila and may also be present in other insect species. 


\section{Introduction}

\subsection{AP axis determination and polarity during insect development}

Evo-Devo field combines areas of developmental biology and evolution. The question of the evolutionary origin of the bilaterian body axes is addressed by investigating the earliest patterning events during development in different bilaterian species. Starting from a single cell, the zygote, the bilaterian body develops with an anterior-posterior (AP) axis, two similar sides (left and right) and an upper and lower surface. The developmental patterning programs that underlie the specification of the body axes have been modified within different bilaterian lineages (De Robertis, 2008).

In most arthropods, the body polarity information derives from cues provided by the mother in form of maternal determinants. Detailed studies in the fruit fly Drosophila melanogaster have given us insight into the developmental mechanisms that pattern the body axes. However, these mechanisms are suspected to have derived from the ancestral mode of axis pattering in arthropods. This derived state is most likely the result of adaptations during development, associated with the rapid embryogenesis. Through comparative analyses of the developmental mechanisms of AP patterning in other species, we are beginning to understand the processes that directed the evolution of body axis patterning in arthropods.

The expression of the homeobox gene caudal (cad/Cdx) is conserved among a wide range of species including nematodes and vertebrates. cad/Cdx genes are consistently expressed in association with posterior polarity and patterning of posterior structures. In Drosophila, cad (Dm'cad) functions as a posterior activator of segmentation genes and is essential for the development of posterior abdominal segments, the anal plates and the hindgut (Macdonald and Struhl, 1986; Mlodzik et al., 1985; Moreno and Morata, 1999; Schulz and Tautz, 1995). In the red flour beetle Tribolium castaneum, Tc'cad is involved in the patterning of posterior head, thoracic and abdominal segments. In Tc'cad RNAi embryos, only the pre-gnathal segments are present, which is in agreement with the $T c^{\prime} c^{\prime}$ ad expression pattern during early development and subsequent expression in the posterior region, the so-called growth zone (Copf et al., 2004). In crustaceans like Artemia franciscana, Af'cad is expressed in the posterior growth zone that gives rise to posterior segments of the developing larvae and is 
essential for the development of posterior thoracic, genital and post-genital segments (Copf et al., 2003; Copf et al., 2004). In the nematode Caenorhabditis elegans the cad/Cdx homologue is called pal-1 and is expressed in the posterior blastomere P1. Loss of pal-1 function results in severe posterior patterning defects (Edgar et al., 2001; Hunter and Kenyon, 1996). In mice, the caudal homologues $C d x 1$ and $C d x 2$ are important in processes of embryonic axial elongation and anterior-posterior patterning (Chawengsaksophak et al., 2004; van den Akker et al., 2002). These observations in different species suggest that $c a d / C d x$ genes had an ancestral role in patterning of posterior segments and body parts.

\subsubsection{AP axis determination in Drosophila melanogaster}

In insects, maternal mRNAs and proteins are deposited into the oocyte to provide positional information during early development. These maternal determinants, depending on their distribution within the embryo, provide differential positional information that will result in the determination of the anterior and posterior ends. Most of our knowledge on AP patterning comes from extensive studies in Drosophila melanogaster, where a gradient system of maternal determinants organizes the patterning of the AP axis in the syncitial blastoderm.

The axial specification occurs in oocyte through polarized microtubules of the cytoskeleton, which leads to the specific localisation of maternal transcripts of bicoid (bcd), oskar (osk), nanos (nos) and gurken (grk) (Steinhauer and Kalderon, 2006). grk is an EGF signalling ligand, essential for the establishment of the anteroposterior and dorsoventral axes (MacDougall et al., 2003), whereas anteriorly localized bcd and posteriorly localized nos and osk specify the AP axis (Becalska and Gavis, 2009).

The graded maternal gene expression stands on top of a gene activation hierarchy, inducing differential activation of segmentation genes along the AP axis of the Drosophila embryo. The protein products of these genes become asymmetrically distributed, creating the network of graded maternal determinants (St Johnston and Nüsslein-Volhard, 1992). The first level of segmentation genes are the gap genes, which become specifically activated by maternal genes (Fig. 2.1, A). Gap genes are the first zygotically expressed genes and specify the body of the embryo into broad domains. Combined activity of maternal and gap genes activate the pair-rule genes, which are expressed in distinct stripes. The expression of the pair-rule genes results in 
expression on the segment polarity genes, establishing the final output of segments (Fig. 2.1, A). Finally, the homeotic genes provide segment identity and determine the functional and morphological fate of each segment (F. Gilbert and R. Singer, 2010; Peel et al., 2005).

Three maternal genetic systems specify the AP axis of the early Drosophila embryo. The terminal system ensures specification of the unsegmented, anterior and posterior most part of the embryo. This specification depends on Torso (Tor), a receptortyrosine-kinase that becomes activated at the poles of the embryo. At the posterior, Tor target genes tailless (tIl) and huckebein $(h k b)$ are activated by relief of repression. At the anterior, Tor and the anterior maternal determinant BCD function antagonistically and cooperatively to activate segmentation gene expression (Furriols and Casanova, 2003; Li, 2005). The anterior system ensures the localization of maternal bcd mRNA at the anterior pole of the oocyte; genes of the posterior system promote localization of nos mRNA at the posterior pole of the oocyte. In the embryo, translation from these localized mRNA sources form two opposing concentration gradients that provide the symmetry breaking information and subsequent activation of segmentation genes (St Johnston and Nüsslein-Volhard, 1992). In contrast, maternal mRNAs of hunchback $(h b)$ and caudal (cad) are provided uniformly in the early embryo, while their protein products are specifically produced in the anterior (HB) and posterior (CAD) through translational repression (Cho et al., 2006). The translational repression of cad mRNA is dependent of $B C D$ activity and results in the posterior-to-anterior gradient of the CAD protein (Dubnau and Struhl, 1996; Rivera-Pomar et al., 1996) (Fig. 2.1, B), whereas $h b$ mRNA is translationally repressed through NOS and Pumilio proteins (Murata et al., 1995; Gamberi et al., 2002; Cho et al., 2006). CAD contains a homeodomain and is essential for the expression of posterior gap genes and morphogenesis of posterior structures. The maternal and zygotic contributions of cad are partially redundant and only embryos mutant for both maternal and zygotic cad expression show severe segmentation effects (Macdonald and Struhl, 1986; Mlodzik et al., 1985; Moreno and Morata, 1999; Schulz and Tautz, 1995). 
A
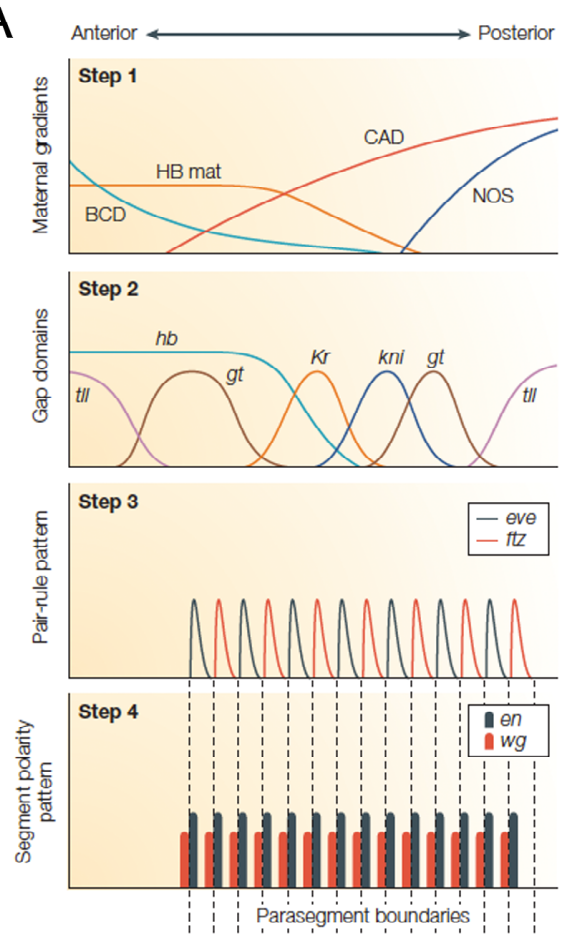

B

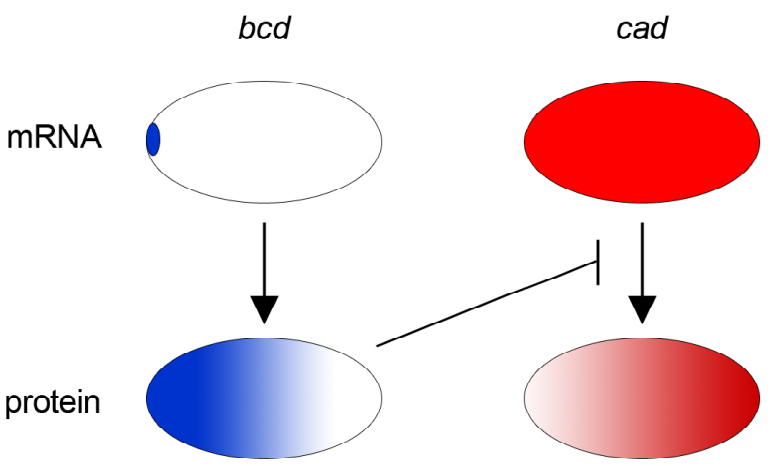

Fig. 2.1: AP axis pattering and segmentation gene cascade in Drosophila melanogaster. (A) Schematic overview of the gene cascade that leads to the formation of body segments along the AP axis. Step 1 includes the differential distribution of maternal genes along the AP axis. bcd and nos are localized as mRNAs at the anterior and posterior pole, respectively, whereas cad and $h b$ mRNAs are distributed homogeneously. Upon egg laying the translation of protein products of bcd and nos results in the formation of two opposing concentration gradients. Besides being a transcription factor, $B C D$ also represses the translation of $C A D$ in the anterior, whereas NOS prevents translation of $h b$ transcripts posteriorly. The coordinates created by the resulting concentration gradients along the AP axis results in the division of the embryo into smaller domains, which are marked by the expression of the gap genes. The gap genes tailless (tll). hb, giant ( $g t)$, Krüppel $(K r)$ and knirps (kni) specify broad domains along AP body axis, which are cross-regulated between them (step 2). Together with the maternal genes, the gap gene coordinates are transformed into the periodic expression of the pair-rule genes hairy, runt and even-skipped (eve) (step 3). The expression of these primary pair-rule genes, together with secondary pair-rule genes like fushi-tarazu (ftz) and paired (prd) are cross regulating to define the boundaries of the parasegments. The parasegments are distinguished into even and odd-numbered units, which are marked by the expression of different pair-rule genes. This expression pattern results in expression of the same segment polarity genes in every parasegment (step 4). The expression of engrailed (en) marks the anterior border of each parasegment and the neighbouring wingless $(w g)$ expressing cells are posterior to every parasegment boundary. Segment boundaries are later marked by en at the posterior border of each segment. (B) Schematic overview of bcd as a translational repressor. bcd mRNA is localized at the anterior pole of the embryo and forms a protein gradient as embryogenesis begins, with highest concentrations at the anterior and lowest at the posterior. cad mRNA is distributed uniformly in the embryo, however due to translational repression by $B C D$, the CAD proteins form a gradient with highest concentration at the posterior. (A taken from Peel et al., 2005) 


\subsubsection{AP axis determination in other insects}

A major evolutionary acquisition of Drosophila and some other holometabolous insect species is the patterning of all body segments during the syncitial blastoderm stage (Peel, 2008). This mode of development is often referred to as "long-germ development" and is thought to have evolved along with changes in extraembryonic tissue formation and underlying molecular networks of axial patterning systems. However many insects, like Tribolium castaneum, pattern only the anterior most segments during the early blastoderm stages and posterior segments are added sequentially after cellularization from a posterior patterning zone. In this mode of "shortgerm development", the embryo proper initially occupies only a small region at the posterior end of the egg and elongates as new segments appear sequentially (Fig. 2.2, A) (Schröder et al., 2008). In fact most arthropod species develop using this mode of sequential segmentation and it is therefore thought to represent the ancestral form of early development (Peel et al., 2005; Rosenberg et al., 2009).

The acquisition of $b c d$ as a maternal anterior determinant is thought to have occurred in a small group within the diptera, specifically in the Cyclorrhapha (Stauber et al., 2002). Therefore, the anterior patterning network with bcd as the main anterior patterning factor during Drosophila development is a derived state and poses the question of how anterior-posterior patterning is realized in other insect species. Here I describe some examples of other insect species that pattern the AP axis independently of $b c d$.

In the wasp Nasonia vitripennis, the embryos pattern all segments at the same time during the blastoderm stage through maternal gradient systems. A bcd orthologue is not present in Nasonia. Anterior patterning is dependent on maternal expression of otd1, which is localized as mRNA at both poles of the embryo. Later on during development, gradients of OTD1 protein are established at both poles of the embryo (Lynch et al., 2006). In contrast to the anterior translational repression of cad as it is known in Drosophila, Nasonia has evolved a different strategy to restrict Nv'cad function to the posterior. Maternal Nv'cad mRNA itself is localized at the posterior pole of the egg resulting in a posterior-to-anterior gradient of Nasonia CAD protein. Whereas the role of Drosophila cad seems to be more restricted, in Nasonia lack of cad activity results in loss of most thoracic and abdominal segments, placing it on top of a cascade of early posterior patterning (Olesnicky et al., 2006). 
It seems that divergent long-germ insects like Nasonia and Drosophila use similar longrange gradients to establish AP pattern, however the specific factors and regulatory mechanisms that establish these gradients are not widely conserved. In the fly Episyrphus balteatus (Syrphidae), which belongs to the Cyclorrhapha, the maternal regulation of Episyrphus caudal (Eba'cad) seems to depend on a combinatory regulation by Eba'BCD and Eba'TOR, though it is not clear, whether Eba'TOR has a direct effect on Eba'BCD activity or whether Eba'TOR and Eba'BCD together could regulate an unknown repressor of Eba'cad translation (Lemke et al., 2010). Thus it is not entirely clear how Eba'cad translational regulation is mediated in Episyrphus.

During early Tribolium embryogenesis, the embryo develops from a syncitial blastoderm, where nuclei aggregate at the posteroventral side to form the embryo proper and anterior nuclei shape the extraembryonic tissues, namely the amnion and the serosa (Schröder et al., 2008). Tribolium does not possess a bcd homologue and anterior patterning seems to be dependent on the function of at least two maternal determinants, Tribolium orthodenticle-1 (Tc'otd1) and Tribolium hunchback (Tc'hb). In the absence of Tc'otd1 and Tc'hb, Tribolium embryos fail to develop head, thoracic and anterior abdominal segments, which is reminiscent of severe bcd mutants in Drosophila (Schröder, 2003).

Two other maternal genes carry out the repression of uniform maternal Tc'cad mRNA in Tribolium embryos. Tribolium zen-2 (Tc'zen-2) and Tribolium Mex-3 (Tc'Mex-3) are expressed in the serosa and anterior head region, respectively, and have been shown to restrict TC'CAD protein from these anterior regions by RNAi analysis (Schoppmeier et al., 2009). In C.elegans, the $\mathrm{KH}$-domain protein MEX-3 is required to restrict the cadlike homeodomain protein PAL-1 to the posterior blastomeres (Draper et al., 1996; Hunter and Kenyon, 1996). Anterior repression of Tc'cad mRNA by Tc'MEX-3 was therefore suggested to be an ancient patterning mechanism that was taken over by bcd in the lineage of higher dipterans (Schoppmeier et al., 2009).

Very little is known about the origins of polarity and the mechanisms that set up the localization and expression of maternal mRNAs in Tribolium embryos (Lynch et al., 2010; Peel and Averof, 2010). Because of the connection between the evolution of caudal regulation and the evolution of $b c d$ and its function as a translational regulator, it is important to assess the role of $b c d$-related genes during insect embryogenesis. Both genes Tc'zen-2 and Drosophila bcd stem from a Hox3/zen-like progenitor (Fig. 2.2, B). First evidence for a common underlying mechanism responsible for the regulation of caudal in Tribolium and Drosophila came from experiments performed by Wolff et al. 
(1998), where Tc'cad transcripts expressed in Drosophila transgenic embryos were translationally repressed in a BCD-dependent manner. The zen genes in Tribolium, however, are not maternally expressed (Falciani et al., 1996; Dearden et al. 2000). Anterior repression of $T c^{\prime}$ 'cad mRNA seems to depend therefore on zygotic activity of Tc'zen-2.

Tc'zen-2 has a sister gene, Tc'zen-1. Both genes arose through a gene duplication event from a Hox3/zen-like progenitor in the lineage of Tribolium (Fig. 2.2, B) and share overlapping expression patterns (Brown et al., 2002; Falciani et al., 1996). Tc'zen-1 acts early in development and specifies the extraembryonic serosa. Lack of Tc'zen-1 activity results in loss of serosa and an expanded posterior germband. The loss of the anteriormost cells can be compensated by the embryo, which then develops normally. Tc'zen-2 has more severe effects, as it is also necessary for the fusion of the extraembryonic tissues amnion and serosa, which is required for dorsal closure. Embryos that fail to express Tc'zen-2 close ventrally and take on an "inside-out" topology (van der Zee et al., 2005). The early role in anterior patterning of Tc'zen-1 was interpreted as a favourable condition for the evolution of bcd (van der Zee et al., 2005). Furthermore is was proposed that the acquisition of an mRNA localization signal in the 3'UTR and the presence of a pre-existing anterior localization mechanism lead to the evolution of bcd (Bucher et al., 2005). 


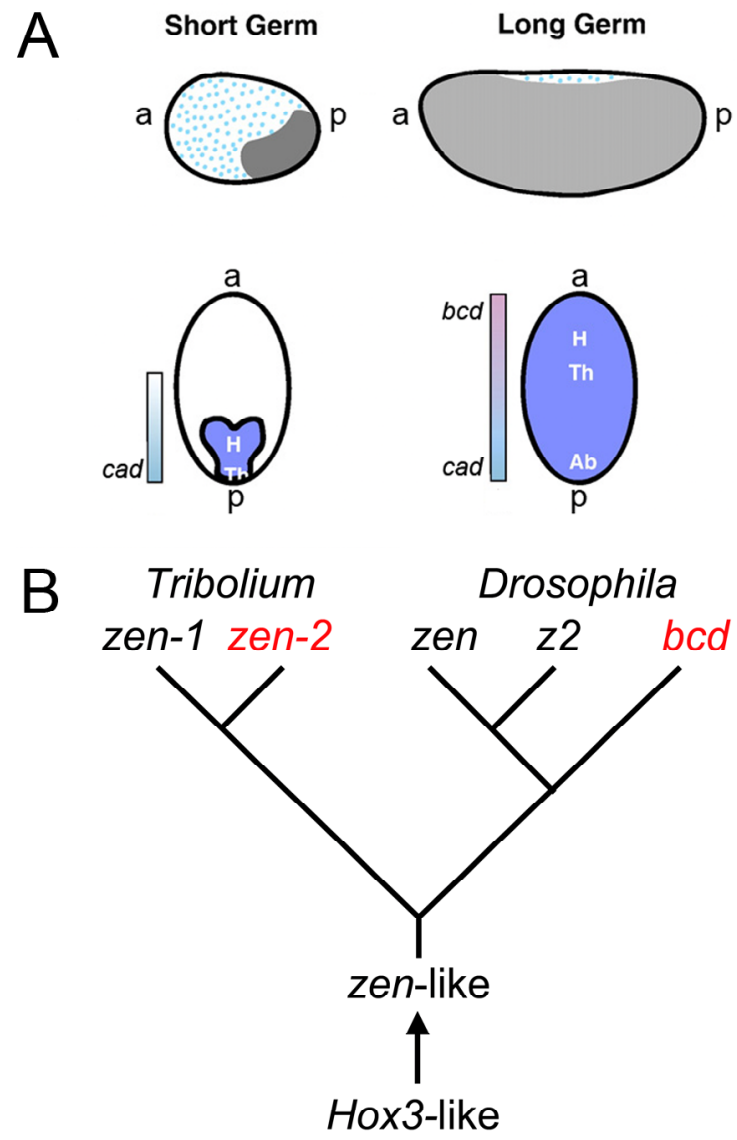

Fig. 2.2: Long-germband development vs. short-germ development and the evolution of Hox3-like genes in the lineage of Tribolium and Drosophila. (A) In the short-germband embryo, the embryo proper occupies a small region at the posterior part of the egg (grey), whereas the rest gives rise to extraembryonic tissue (blue dots). The body segments that are patterned in this blastoderm are the head segments $(\mathrm{H})$ and thoracic segments (Th) (ventral view in blue); posterior segments will be added progressively from a posterior growth zone. In the long-germ embryo, most of the blastoderm will give rise to the embryo proper (grey) and the segments of the head $(\mathrm{H})$, thorax $(\mathrm{Th})$ and abdomen $(\mathrm{Ab})$ are specified simultaneously (ventral view in blue). Tribolium forms a short germ, whereas Drosophila forms a long germ. (B) The common ancestor of Tribolium and Drosophila most likely owned one Hox3-like gene of the zen-type. In the lineage that lead to Tribolium, this zen-like progenitor duplicated giving rise to two zen homologues, zen-1 and zen-2. In the lineage of Drosophila, a gene duplication resulted in appearance of the zen-type gene progenitor and the bcd-type gene progenitor. The bcd progenitor evolved into the $b c d$ gene, the zen-type progenitor duplicated again and gave rise to the two zen-orthologues, zen and z2. (A modified after Rosenberg et al., 2009). 


\subsection{The anterior patterning factor Bicoid}

\subsubsection{Evolution of Bicoid}

$b c d$ and zen are sister genes belonging to the Hox-gene cluster and have evolved after a gene duplication from a Hox3-like ancestor (Falciani et al., 1996; Stauber et al., 1999). The gene duplication that gave rise to the progenitors of bcd and zen occurred in the diptera, as bcd orthologues have only been found in flies that belong to the Cyclorrhapha (Fig. 2.3) (Lemke et al., 2008; Stauber et al., 2000). Both genes have evolved much more rapidly then their neighbouring Hox genes (Falciani et al., 1996; Sommer and Tautz, 1991; Stauber et al., 1999) and have acquired different functions during early development. In the lineage leading to Drosophila melanogaster a second gene duplication gave rise to the two orthologues zen and $z 2$. Both genes share identical expression patterns, however as deletion experiments showed, only zen can provide full zen gene function (Pultz et al., 1988; Rushlow et al., 1987a).

In Drosophila, bcd is required for the patterning of the head and thorax, while zen is involved in the specification of the extraembryonic tissue anlagen (amnioserosa). bcd is a maternal gene, expressed only in the nurse cells and deposited as mRNA into the oocyte, where it becomes localized anteriorly. zen is zygotically expressed in a broad region at the dorsal side of the embryo and retracts later into a narrow dorsal domain to specify the amnioserosa (Bate and Martínez Arias, 1993; Rushlow et al., 1987a; Rushlow et al., 1987b).

The maternal expression of bcd homologues is conserved among different cyclorrhaphan flies, whereas maternal expression of the zen progenitor was lost in the lineage of Cyclorrhapha. Stauber et al. (2001) have analysed the expression patterns of Hox3'zen-like orthologues from several non-cyclorrhaphan dipterans and found that the identified gene sequences are more similar to the zen-type gene. However they share expression patterns similar to both bcd and zen homologues (Stauber et al., 2002). 


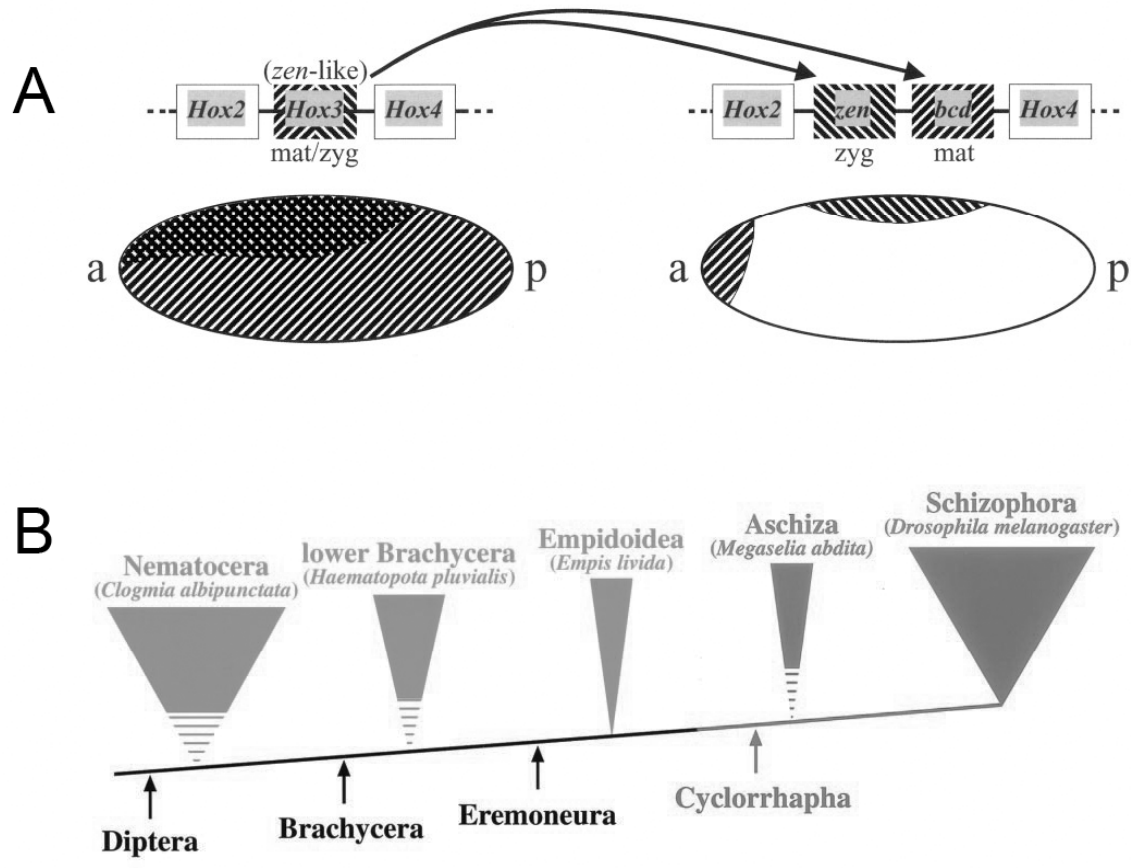

Fig. 2.3: Schematic overview of putative evolution of bcd and zen in taxa of diptera. (A) A gene duplication of a Hox3/zen-like gene with putative combined maternal and zygotic expression patterns gave rise to the sister genes zen and bcd. (B) This duplication occurred in the lineage of the cyclorrhaphan flies (indicated by arrow). The Nematocerca (like Clogmia albipuctata), Brachycerca (like Haematopota pluvialis) and Aschiza (like Megaselia abdita) are most likely paraphyletic groups, whereas the monophyly of the taxa Empidoidea (like Empis livida) and Schizophora (like Drosophila melanogaster) is well supported (Yeates and Wiegmann, 1999). (Taken from Sander and Schmidt-Ott, 2004; Stauber et al., 2002).

\subsubsection{Function of Bicoid}

The full-length Bicoid protein consists of 494 amino acids, including several functional domains that are indicated in Fig. 2.4. BCD is a special transcription factor because it has a dual function in DNA-binding where it acts as a transcription factor and in RNAbinding where it acts as a translational repressor (Dubnau and Struhl, 1996; RiveraPomar et al., 1996; Struhl et al., 1989). With its K50-homeodomain it binds to the DNA consensus sequence TAATCC (Driever and Nüsslein-Volhard, 1989). The residue with key role in target specificity is a lysine at position 50 in the third helix of the homeodomain. Position 54 is occupied by an arginine and this modification is unique to the homeodomain of BCD (Mcgregor, 2005). The arginine-rich motif in the homeodomain and specifically R54 is crucial for RNA-binding during translational repression of cad mRNA (Niessing et al., 2000). Two other domains of the BCD protein have been reported to be involved in translational repression. The d4EHP-binding 
domain, through which BCD directly binds to the 5'-cap binding factor d4EHP (Cho et al., 2005). and the PEST domain (amino acids 170-203) (Niessing et al., 1999).

Fig. 2.4 shows additional regions of the BCD protein that have been identified and found to influence its activity as a transcriptional activator (Janody et al., 2001; Schaeffer et al., 1999; Zhao et al., 2002).

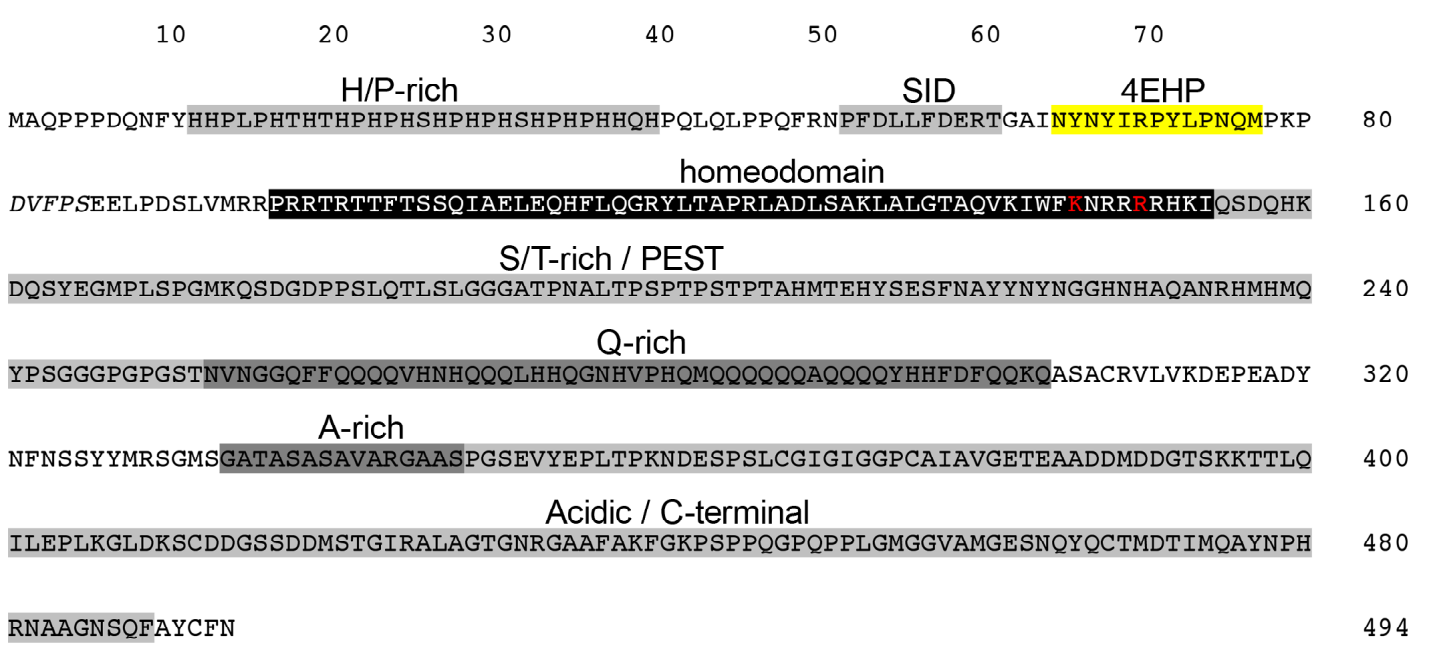

Fig. 2.4: The protein domains of BCD isoform G. The BCD protein is a polypeptide of 494 amino acid residues and the homeodomain is indicated in black and the lysine and arginine at position 50 and 54 highlighted in red. The self-inhibitory domain (SID) spanning at least aa 5261 repressed BCD activity in S2 cells (Zhao et al., 2002). The 4EHP-domain (4EHP, indicated in yellow) and the PEST domain have been shown to be invovled in translational regulation of cad mRNA (Cho et al., 2005; Niessing et al., 1999). In cell culture, the serine/threonine rich domain (S/T-rich, aa 152-252), the glutamine-rich domain (Q-rich, aa 253-300), and acidic C-terminal domain (Acidic/C-terminal aa 349-489) act as activation domains, whereas the alanine-rich domain (A-rich, aa 334-348) seems to be a repression domain. The function of the histidine/proline-rich domain (H/P-rich, aa 11-42) remains elusive. Furthermore, the S/T-rich domain and the $\mathrm{C}$ domain mediate the downregulation by Torso (Janody et al., 2001; Schaeffer et al., 1999).

\subsubsection{The Bicoid isoforms}

The bcd gene generates five mRNAs through alternative splicing of four major exons (termed exon 1-4, Fig. 2.5). Exon 1, present in all isoforms, encodes the 5'UTR and a histidine/proline-rich repeat (H/P-rich) at the $\mathrm{N}$-terminus. Exon 2 encodes the selfinhibitory domain (SID) and the 4EHP-binding domain, exon 3 encodes the homeodomain, the serine/threonine-rich domain (S/T-rich), a glutamine-rich domain (Q- 
rich) and an alanine-rich domain (A-rich). The acidic C-terminal domain (Acidic) is encoded in part by the exons 3 and 4 . Exon 4 also contains the 3'UTR and is present in all isoforms. The presence of alternative splice acceptor sites on exons 2 and 3 results in isoforms containing slightly longer or shorter versions of exon 2 ( $2 a$ or $2 b$ ) and exon 3 (3a or $3 b$ ), respectively (Fig. 2.5).

mRNA isoforms $G$ and $D$ code for the longest protein products. Whether the mRNA isoforms $\mathrm{E}$ and $\mathrm{F}$ result in their respective protein products is unclear because of an in frame stop codon in exon 2a (Fig. 2.5, indicated by arrows). A putative ORF could start form a start codon that lies further downstream in exon 2a (Fig. 2.5, indicated in red; Fig. A5.1). Isoform A, which has the shortest ORF of all isoforms consists only of exon 1 and 4.

Most studies refer to isoform $G$ as the wt BCD protein, however, to date, the existence of bcd splicing variants has been largely ignored and it is unclear to want extent each isoform contributes to BCD function during embryogenesis. Interestingly, the putative protein isoforms $E$ and $F$ contain all domains crucial for transcriptional activation of target genes, but are lacking the 4EHP-binding domain implicated in the 5'-cap structure mediated translational repression of the cad mRNA (Cho et al., 2005) (Fig. 2.5). The bcd gene has five annotated transcripts, of which all isoform cDNAs have been recovered from ESTs of Drosophila embryonic libraries; only BCD isoform $D$ and $G$ are annotated as fully sequenced cDNA clones. There are indications from RNA sequencing expression profiling that at least the transcripts of isoform $G, D, E$ and $F$ are all present in the early embryo (Tweedie et al., 2009)(Fig. A5.2). 
bcd isoforms

\section{Exon 1}

Exon 2

Exon 3

Exon 4

G

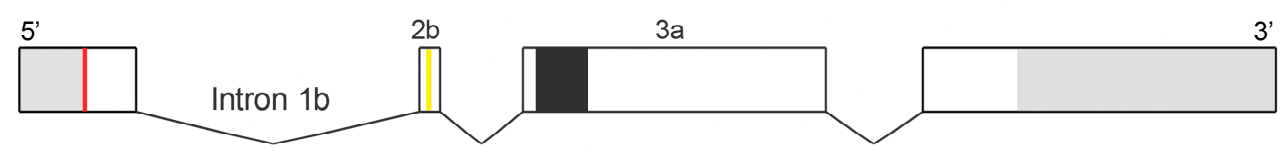

D

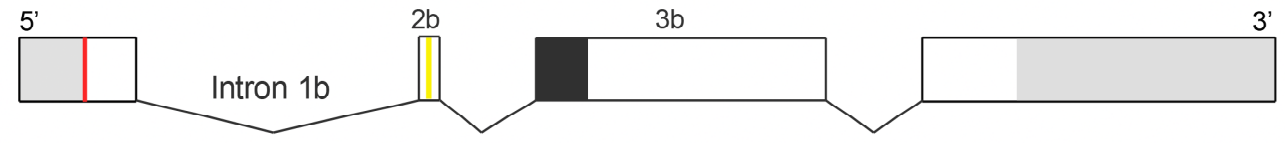

E

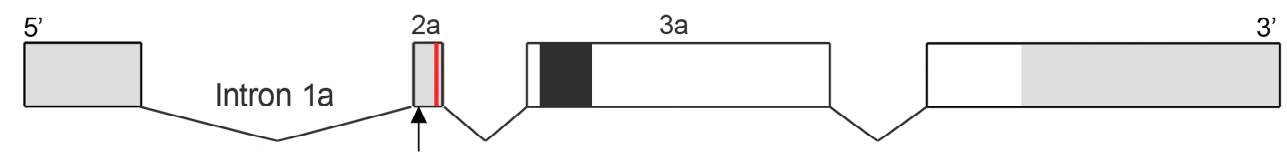

F

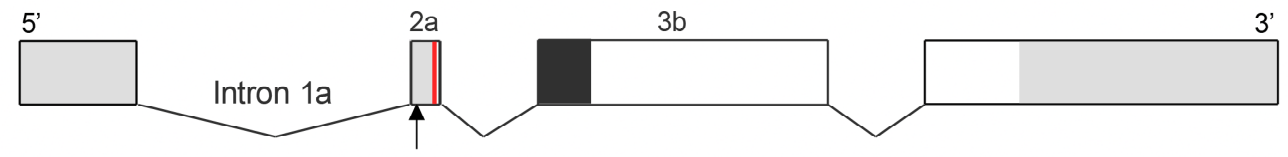

A

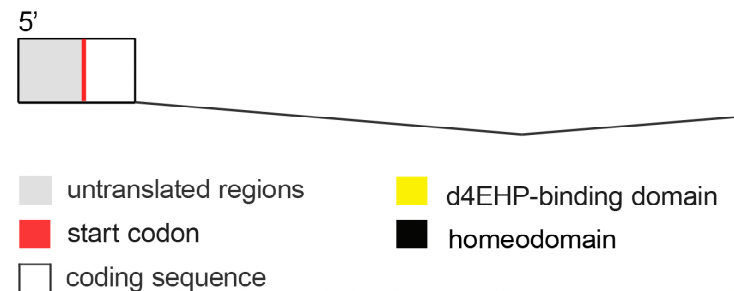

Fig. 2.5: Schematic overview of the bcd transcripts produced by differential splicing of four major exons. Exon 1 and 4 are present in all isoforms. Alternative splicing with two splice acceptors at the 5' end of exon 2 results in the presence of either exon $2 a$, which contains an in frame stop codon (arrows) and is thought to initiate translation on a downstream AUG. Exon $2 b$ maintains the ORF from the upstream AUG in exon 1. Alternative splicing with two splice acceptors at the $5^{\prime}$ end of exon 3 results in the presence of exon $3 a$ or $3 b$, which differ in their capacity to encode a short peptide sequence (DVFPS). Untranslated regions are marked in grey, the coding sequences are marked in white. The 4EHP-binding domain is indicated in yellow, the homeodomain is indicated in black.

\subsection{Mechanism of cad translational regulation in Drosophila}

Maternal cad mRNA is distributed uniformly in the oocyte and early Drosophila embryo. The formation of the BCD AP gradient generates an opposing gradient of the CAD protein (Fig. 2.1, B). In this process, BCD directly binds to specific region(s) in the cad 3'UTR to mediate the translational repression of the cad transcripts. The BCD RNAbinding property stems from its K50/R54-homeodomain, which is capable of binding directly to BCD-binding element(s) (Chan and Struhl, 1997; Dubnau and Struhl, 1996; 
Rivera-Pomar et al., 1996). The dual DNA and RNA-binding function of BCD depends on two amino acid residues ( $\mathrm{K} 50$ and $\mathrm{R} 54$ ) in the third helix of the homeodomain that, in this combination, are unique to BCD (Niessing et al., 2000). The 3'UTR element to which BCD binds has been characterized biochemically and genetically and includes a $120 \mathrm{nt}$ and a $323 \mathrm{nt}$ fragment of the cad 3'UTR (Dubnau and Struhl, 1996; RiveraPomar et al., 1996) (see Fig. 3.1). When the $323 \mathrm{nt}$ fragment is removed from the cad 3'UTR, CAD becomes ectopically expressed in the anterior part of the embryo (Dubnau and Struhl, 1996; Rivera-Pomar et al., 1996). A specific homeodomain/RNA recognition motif as not yet been identified.

For the translational repression of cad transcripts, BCD requires direct interaction with Drosophila 4E homolog protein (d4EHP) through its d4EHP-binding domain. d4EHP is a elF4E-related protein, which binds directly to the 5'-cap structure of the mRNA, but has lost it's ability to interact with elF4G (Hernández et al., 2005). The simultaneous interaction of BCD with the 3'UTR and the cap-associated d4EHP renders the cad mRNA translationally inactive. The mechanism of d4EHP-dependent BCD-mediated translational repression of cad has been proposed as a new paradigm for translational control (Cho et al., 2005) (Fig. 2.6).

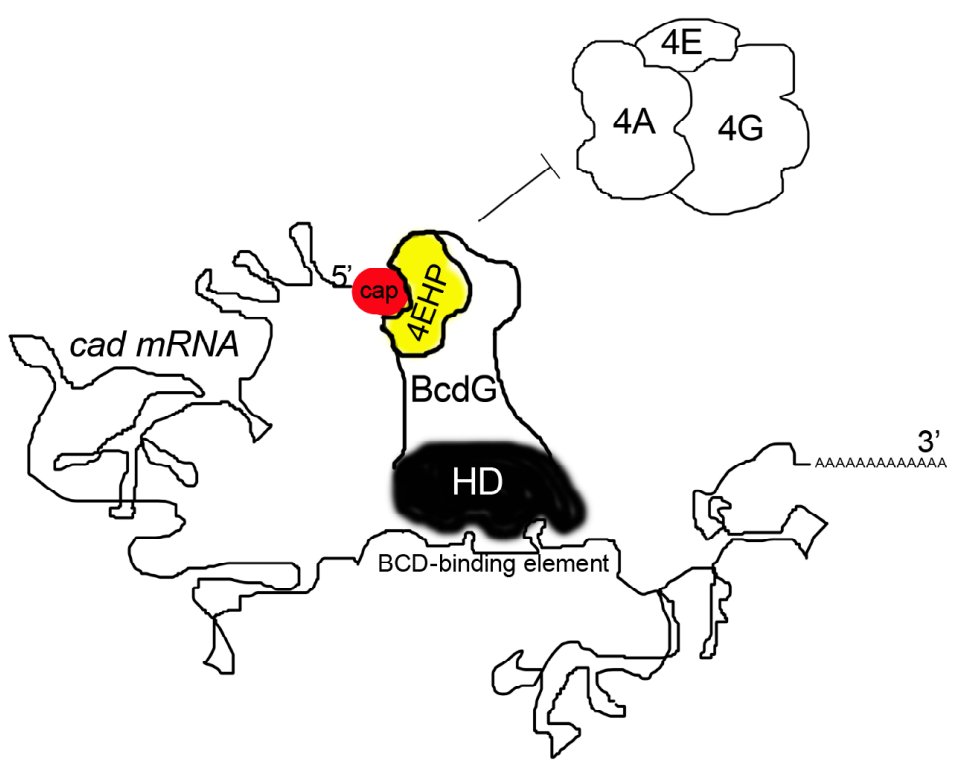

Fig. 2.6: Model of cad translational repression, proposed by Cho et al. (2005). BCD binds via its homeodomain (black) to the BCD-binding element(s) in the 3'UTR of the cad mRNA and at the same time interacts with 4EHP (yellow), which binds to the 5' cap structure (red). Hence, the translational initiation factors are prevented from binding to the cad mRNA and remains untranslated. (Modified after Cho et al., 2005). 


\section{4 miRNA function during early Drosophila development}

During this $\mathrm{PhD}$, the analysis of cad 3'UTR elements that mediate BCD-dependent translational repression lead me to investigate the putative role of microRNAs (miRNAs) in cad translational regulation. miRNAs are short ( 22 nt) non-coding RNAs. They have gained increasing recognition in the post-transcriptional regulation of gene expression in animals and plants. In animals, miRNAs interfere with mRNA translational and/or stability through imperfect base pairing to the 3'UTR of their target mRNAs. Target-site recognition requires complementarity of the miRNA 5'-region, which is called the seed sequence, with sequences in the target mRNA. Incorporated in the RNAi induced silencing complex (RISC), miRNAs guide the RISCs to their targets in a sequence specific manner and mediate translational control (Ameres et al., 2007; Jackson and Standart, 2007; van den Berg et al., 2008). The target mRNAs become translationally repressed or subjected to degradation by RISC, depending on the degree of sequences complementarity of the miRNA to the mRNA (Bartel, 2004; Carrington and Ambros, 2003). The core proteins of RISC belong to the protein familiy of Argonaute (Ago) proteins. In Drosophila, the Ago proteins Ago1 and Ago2 associate with different types of small RNAs (miRNAs and siRNAs) which dictates their functions. Ago1-RISC mediates translational repression of the target mRNA by depolyadenylation of the poly(A)-tail, whereas Ago2-RISC blocks the functions of the capstructure (Iwasaki et al., 2009). Translational repression by Ago1 and Ago2 are therefore mechanistically different. However, it seems that translational repression by RISC is put into effect through various mechanism acting on different steps of translational initiation (Chendrimada et al., 2007; Humphreys et al., 2005; Kiriakidou et al., 2007; Mathonnet et al., 2007; Pillai et al., 2005; Thermann and Hentze, 2007).

For a number of miRNAs in Drosophila, the expression profiles have been identified and their role in development has been analysed during early development (Aravin et al., 2003; Leaman et al., 2005). Injections of 2'O-methyl oligoribonucleotides with complementary to miRNAs into early Drosophila embryos demonstrated the involvement of miRNAs in processes like cellularization, segmentation, dorsal closure and apoptosis. (Leaman et al., 2005).

The cad 3'UTR harbours two putative miRNA target sites that have been predicted computationally; the target sequence of miR-308 is located in region nt 299-316 and the miR-305 target sequence in region nt 534-545 in the cad 3'UTR. (Betel et al., 2008; Brennecke et al., 2005; Lai et al., 2003; Stark et al., 2005). Both miR-308 and miR-305 
have been cloned from embryonic tissues, however neither of these two miRNAs has been detected by Northern blotting analysis. For miR-308, 4 independent clones have been isolated from a cDNA library of $0-2 h$ of embryogenesis and 1 clone from a cDNA library of 2-4h of embryogenesis. For miR-305 there has been only 1 clone isolated from a cDNA library of $0-2 \mathrm{~h}$ embryogenesis (Aravin et al., 2003). A function during development has been reported for miR-308 in control of apoptosis during embryogenesis. Embryos that are depleted of miR-308 activity show a mild increase in apoptosis during mid-embryogenesis (Aravin et al., 2003; Leaman et al., 2005).

\subsection{Rationale}

In the investigation of the anterior patterning factor(s) in Tribolium, Wolff et al. (1998) conducted a crucial experiment, in which they expressed the Tribolium cad homologue in Drosophila embryos. Surprisingly, the Tribolium transcripts were translationally regulated in a BCD-depended manner. Tribolium itself though does not possess a bcd homologue. It was inferred that the Tc'cad 3'UTR sequence most likely contained a conserved binding element that could be recognized by BCD.

Eventually, more than ten years later, the translational regulators of the Tc'cad mRNA have been identified as Tc'zen-2 and Tc'Mex-3 (Schoppmeier et al., 2009). The putative binding elements in the Tc'cad 3'UTR to which Tc'ZEN-2 and Tc'MEX-3 bind have not been identified yet. Tc'MEX-3 is a $\mathrm{KH}$-domain protein and might require different sequences for RNA-binding than BCD. TCZEN-2, however, is phylogenetically related to $B C D$.

Experiments with a cad homologue from the dipteran Megaselia abdita have shown that Drosophila BCD does not translationally regulate the Mab'cad 3'UTR, although a Megaselia bcd homologue is present in this species. Interestingly, Mab'cad mRNA is not maternally expressed as its homologues in Drosophila or Tribolium (Stauber et al., 2008).

These findings have posed some questions. Can BCD bind to a wide range of cad 3'UTR homologues and what are the requirements for BCD-recognition? What does the binding element of the Tc'cad 3'UTR, to which BCD can bind consist of and can we find any similarities to the BCD-binding element(s) in Drosophila? 
The characterization of the cad 3'UTR homologues that are recognized by BCD and the detailed analysis of the BCD-binding element in Drosophila can help to gain further insight into when and how cad translational repression arose during evolution.

The aim of this study was to establish an in vitro assay using bacterially expressed BCD homeodomain and electrophorectic mobility shift assays for the detailed mapping of BCD-binding elements in the 3'UTR of different cad homologues. In a parallel approach, I aimed to establish an in vivo reporter assay, to test these BCD-binding elements in their capability to mediate BCD-dependent translational repression in living embryos. 


\section{Results and Discussion}

\subsection{Mapping of BCD-binding RNA-elements in vitro}

Several studies have shown in vitro evidence for direct binding of the BCD homeodomain to distinct elements within the 3' UTR of cad. These elements range from nt 67 - 186 (BBR, Rivera-Pomar et al., 1996), nt 210 - 318 and nt 319 - 433 (termed BRE fragments B and C, Dubnau and Struhl, 1996) of the cad 3'UTR (Fig. 3.1). The initial experiments from Dubnau and Struhl could not be reproduced (Dubnau and Struhl, 1997), however it was possible to confirm sequence-specific binding of BCD to a 3'UTR fragment containing nt 210 - 253 using different conditions (Chan and Struhl, 1997).

BCD also seems to recognize mRNA sequences from the Tribolium cad homologue in transgenic Drosophila (Wolff et al., 1998), although in Tribolium the early regulation of maternal transcripts is taken over by other factors, such as Tc'mex-3 and Tc'zen-2 (Schoppmeier et al., 2009). The element that provides the BCD-dependent signal in the Tribolium cad mRNA has not been identified yet, but it is likely to be situated in the 3'UTR.

The horsefly Haematopota pluvialis, a dipteran and like Drosopihla belonging to the group of the brachycerca, does not posses a BCD homologue (Stauber et al., 2002). Nonetheless, maternal transcripts of the Haematopota cad (Hp'cad) homologue have been found in the nurse cells and oocyte (Stauber et al., 2008), suggesting the need for a mechanism that prevents maternal Hp'cad transcripts from being expressed in the anterior.

Taken together, these observations suggest that $B C D$ may recognize the mRNAs of a range of cad homologues via element(s) that have been conserved in the course of evolution and mediate translational repression of the transcript. In order to identify these conserved elements, I conducted electromobility shift-assays (EMSA) using bacterially expressed $B C D$ homeodomain (HD) and tested the binding of the homeodomain to different in vitro transcribed RNA fragments. 
Dm cad 3' UTR [nt 1 - 862]

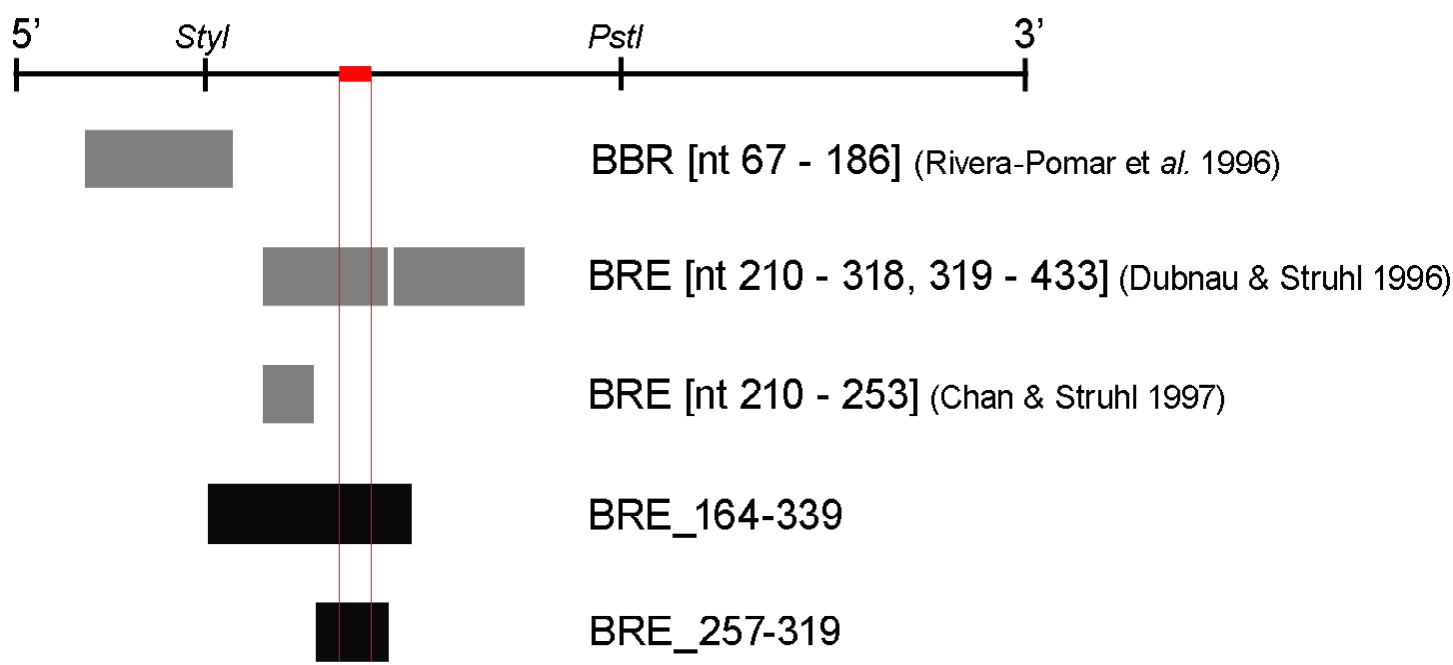

Fig. 3.1: Drosophila cad 3' UTR and RNA fragments previously described as Bicoid binding elements are indicated as grey shaded boxes. RNA fragments used in this study are indicated as black shaded boxes. The predicted miR-308 binding site in the cad 3'UTR is indicated with a red box. Numbering starts with the first nt after the stop codon.

\subsubsection{Establishment of RNA-binding assay using recombinant BCD homeodomain protein}

For the establishment of an RNA-binding assay with the BCD homeodomain I drew information for in vitro binding conditions from previously published work. The direct and specific interaction of the BCD homeodomain with the fragments BRE_210-318, BRE_319-433 of the cad 3'UTR were initially shown by in vitro binding of the BCD homeodomain from bacterial cell lysates to radiolabeled RNA probes and PAGEanalysis (Dubnau and Struhl, 1996). However the competition experiments done in this study were not reproducible. By using different binding conditions, competition experiments with bacterially expressed, purified BCD homeodomain and an RNA probe containing nt 210-253 of the cad 3'UTR were successful and confirmed the RNA sequence-specificity of the BCD homeodomain (Chan and Struhl, 1997). The binding conditions used by Chan and Struhl (1997) where used here as a first reference point.

Rivera-Pomar et al. (1996), using crosslinked RNA from full-length cad mRNA and derivatives to nuclear proteins from Drosophila embryos, demonstrated BCDdependent binding of a 120 nt cad 3'UTR fragment, which was assigned as position 1350-1470 with the first nucleotide of the start codon as position 1 of the cad mRNA. 
This region most likely corresponds to nt 66-185 of the cad 3'UTR (NCBI accession number AY069565), here termed BBR_66-185. However, there have been no attempts to show sequence-specificity of $B C D$ homeodomain binding on this fragment by competition experiments. In subsequent binding experiments with bacterially expressed and purified BCD homeodomain, Rivera et al. (1996) used a 110 nt fragment, which was referred to as the BBR. With this fragment, the RNA-binding vs. DNA-binding capacity of mutagenised BCD homeodomain derivatives were tested (Niessing et al., 2000). An analysis of the sequence-specificity of the BCD homeodomain during RNAbinding was not performed.

Despite several attempts, I was not able to reproduce RNA-binding of bacterially expressed and purified BCD homeodomain using the same conditions as described by Chan and Struhl (1997) or Niessing et al. (2000). I found that the presence of 2,5 $\mu \mathrm{g} / \mu \mathrm{l}$ heparin during the RNA binding reaction was inhibiting complex formation with the bacterially expressed $B C D$ homeodomain (Fig. A1.1, C). I therefore decided to omit heparin from the RNA binding reactions. Furthermore, Chan and Struhl (1997) used 0.1 $\mu \mathrm{g} / \mu \mathrm{l}$ yeast tRNA, whereas Niessing et al. (2000) used $5 \mu \mathrm{g} / \mu \mathrm{l}$ E.coli tRNAs. I found that the presence of $5 \mu \mathrm{g} / \mu \mathrm{l}$ of yeast tRNA inhibits complex formation with the fusion protein HisMBP-HD and a RNA probe spanning nt 164-512 of the cad 3'UTR determined that a concentration of $0.25 \mu \mathrm{g} / \mu$ l yeast tRNAs blocks unspecific binding properties of HisMBP-HD while allowing complex formation with the RNA probe (Fig. $A 1.1, A, B)$. After liberation of the homeodomain from HisMBP, the affinity to the RNAtarget increased, so that $0.5 \mu \mathrm{g} / \mu \mathrm{l}$ of yeast tRNAs were used during the binding reaction.

Because translational repression and transcriptional activation mediated by $B C D$ both depend on the homeodomain, I further tested the functionality of the recombinant protein by binding to a dsDNA target site containing the consensus sequence 5'CTAATCC-3' (Fig. 3.2, lanes 1-4). The binding of the BCD homeodomain to dsDNA was performed under the conditions determined to be best for RNA binding. DNAbinding of the BCD homeodomain is specific, which was also revealed by competition experiments (Fig. A1.2 and Fig. A1.3). This indicates that the bacterially expressed $B C D$ homeodomain is functional. 


\subsubsection{Analysis of recombinant BCD homeodomain binding to RNA}

For RNA binding I chose a 175 nt fragment spanning nt 164-339 of the cad 3'UTR (BRE_164-339, see Fig. 3.1), which contains the BRE_210-318 fragment (Dubnau and Struhl, 1996) and subsequently verified fragment BRE_210-253 (Chan and Struhl, 1997). The homeodomain was purified as a MBP-HD or GST-HD fusion protein. For the binding reaction, 5-40 pmole of the homeodomain were incubated with 300 fmole radiolabeled BRE_164-339 probe and run on a non-denaturing polyacrylamide gel (Fig. 3.2, lanes 5-7). Parallel binding reactions were performed using 300 fmole of an unrelated RNA fragment of similar size (Adh3' 184 nt) (Fig. 3.2 lane 8-10) and 2 pmole of the target dsDNA (Fig. 3.2, lanes 1-4).

The BRE_164-339 runs in two major bands (I. and II.), which is probably due to different structural conformations of the RNA molecule (Fig. 3.2, lane 5). With 40 pmole BCD homeodomain complexes are formed with BRE_164-339 bands I. and II., indicated by a higher running band in the gel (Fig. 3.2, lane 7), whereas the unrelated RNA fragment Adh3' does not shift at similar homeodomain concentrations. The binding affinity for the dsDNA seems to be higher, as the DNA shift occurs already with 2.5 pmole of BCD homeodomain.

To test whether the observed binding to BRE_164-339 is specific, I performed competition experiments using 40 pmole of homeodomain and 300 fmole of radiolabeled BRE_164-339 in the presence of different amounts of unlabeled (cold) specific (BRE_164-339) and unspecific (Adh3') RNA (Fig. 3.3, lane 6-13, respectively). The HD:BRE_164-339 complexes (with both RNA species I. and II.) are strongly inhibited by a 20-fold excess of cold BRE_164-339 and completely absent at the presence of a 100-fold excess (Fig. 3.3, lane 6 and 7). At a 20-fold or 5-fold excess of cold BRE_164-339, the HD:BRE_164-339 complex is competed only slightly more efficiently than at similar amounts of cold Adh3' (Fig. 3.3, compare lane 7 to lane 11 and lane 8 to lane 12). In comparison, the homeodomain binds with high affinity to the dsDNA target, which was observed in DNA-binding and competition experiments (Fig. A1.2 and Fig. A1.3).

It is still unknown how binding to the RNA target occurs and what the minimal RNA binding motif exactly consists of. Using electrophoretic mobility shift assays I aimed to map the exact region(s) of the BCD binding element in the Dm'cad and in 3'UTRs of other cad homologues. I established in vitro binding conditions under which binding of recombinant $\mathrm{BCD}$ homeodomain to its RNA-binding target can occur. I found that 
under native conditions RNA-binding of the bacterially expressed BCD homeodomain to the BRE_164-339 fragment occurs with a very low binding affinity. The low binding affinity to the BRE_164-339 fragment was not expected, since this fragment contains previously identified BCD binding elements (see Fig. 3.1), which were reported to be bound with high specificity by the BCD homeodomain (Chan and Struhl, 1997). Nonetheless, the here described recombinant BCD homeodomain is functional, which I could demonstrate in DNA-binding and -competition experiments. 


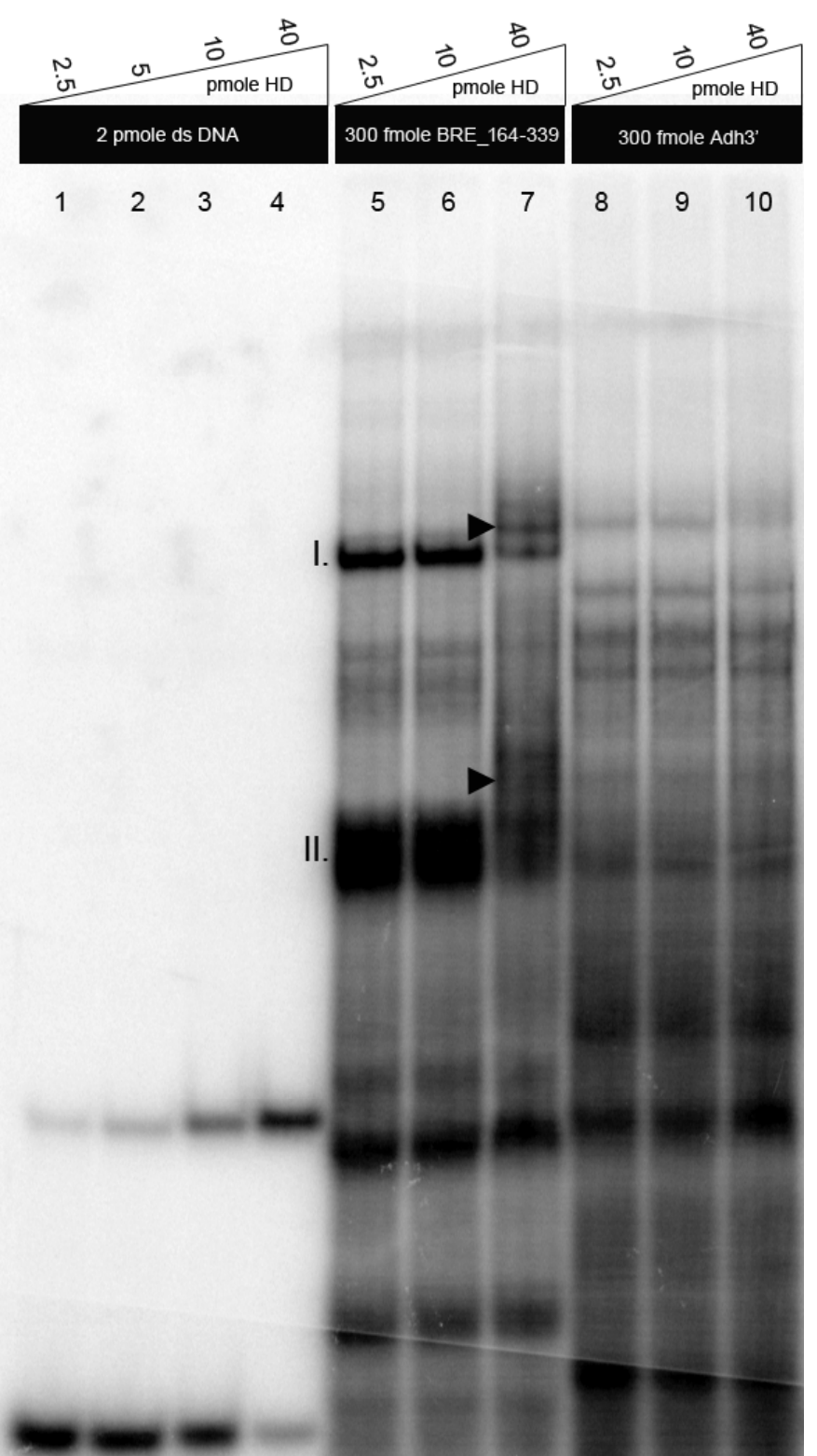

Fig. 3.2: DNA- and RNA-binding assay of BCD homeodomain. Lanes 1-4 show binding to 2 pmole of dsDNA target site at increasing homeodomain concentrations. With 300 fmole of BRE_164-339 probe (lane 5-7), the RNA is shifted at a homeodomain concentration of 40 pmole (lane 7). The RNA molecule of BRE_164-339 exists in two conformations (I. and II.) which are bound by the homeodomain (lane 7 , arrowheads). In contrast, with 300 fmole of Adh3' the homeodomain does not seem to for a complex that migrates at shifted positions in the gel (lane 8-10). 


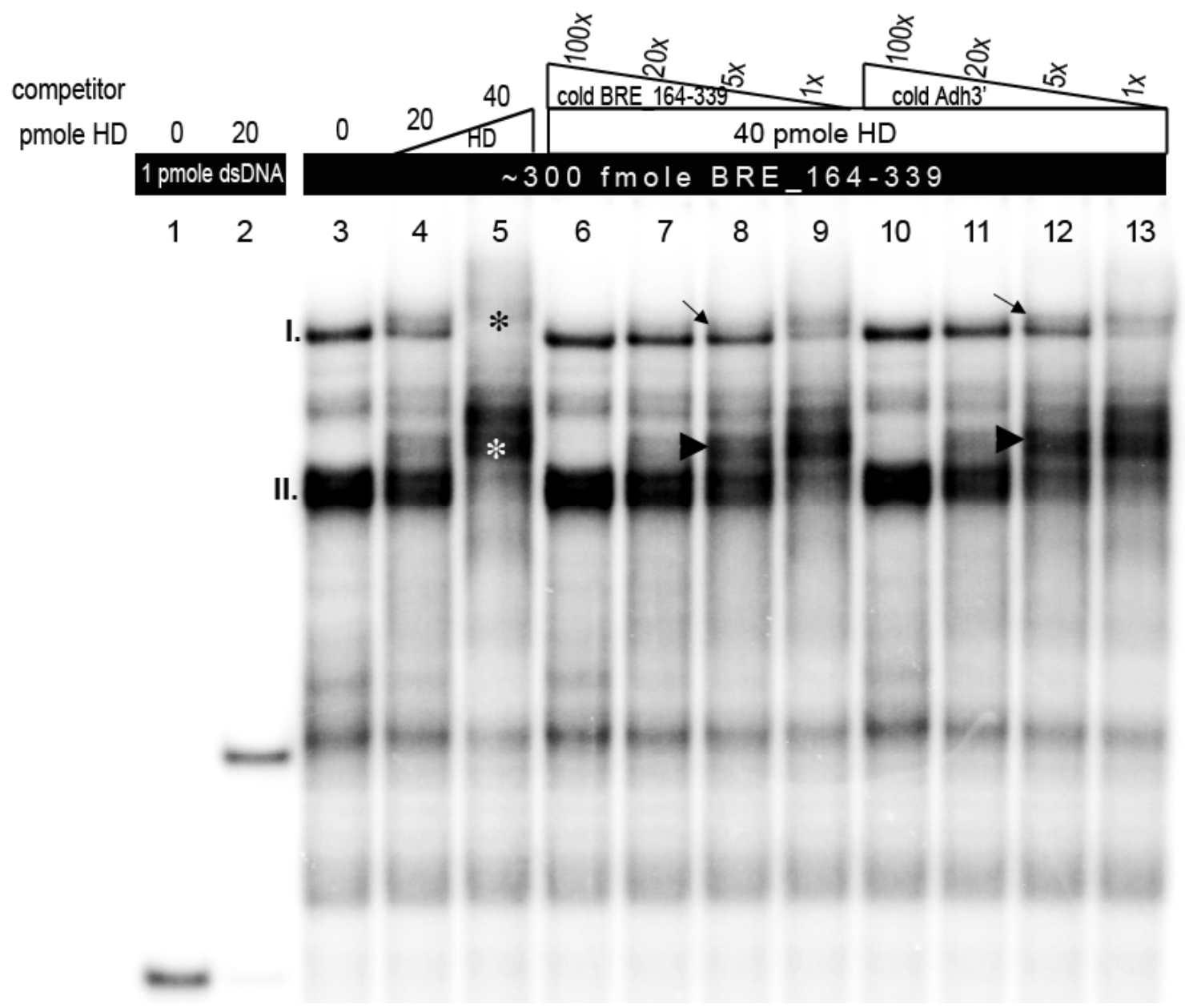

Fig. 3.3: Binding of BCD homeodomain to BRE_164-339. Both complexes of the homeodomain with BRE_164-339 I. and II. (indicated with asterisks in lane 5) are inhibited in the presence of 100-fold excess of cold BRE_164-339 or Adh3' (lanes 6 and 10). At 5-fold excess of cold competitor, complex HD:BRE_164-339 II is less severely inhibited by cold Adh3' (lane 12, arrowhead) than by the specific competitor (lane 8, arrowhead). Also the higher running complex of HD:BRE_164-339 I seems to be less severely inhibited by cold Adh3' (compare lane 8 with lane 12, arrows). Lanes 1-2 show DNA binding as a control for proper functionality of the homeodomain. 


\subsubsection{Characterization of the BCD binding element BRE_257-319 in the Drosophila cad 3'UTR}

So far there have been three different Bicoid binding elements described in the cad 3'UTR (Chan and Struhl, 1997; Dubnau and Struhl, 1996; Rivera-Pomar et al., 1996) (see Fig. 3.1). Alignments of cad 3'UTRs from different Drosophila species do not show extensive sequence similarities among each other, apart from a highly conserved region of 38 nucleotides (Fig. 3.4, nt 271-308 in the cad 3'UTR, region indicated with asterisks, for the full alignment see Fig. A2.1), which is lying within the BRE_210-318 (Dubnau and Struhl, 1996), but outside of the BRE_210-253 fragment studied by Chan and Struhl (1997). Although translational repression of the maternal cad mRNA most likely depends on BCD in all Drosophila species, the overall level of sequence conservation of the previously described fragments BBR_66-185 and BRE_210-253 seems to be very low (Fig. 3.4).

In order to investigate whether the BCD homeodomain is able to bind to the BRE_257319 fragment, I performed band-shift experiments (Fig. 3.5). At the same time I investigated the binding specificity of the bacterially produced homeodomain to RNA fragments with different structural qualities. The shSV40 fragment $(79 \mathrm{nt}$ from the SV40 3'UTR) is predicted to form several secondary structures (not shown). The CU58mer fragment, a $\mathrm{CU}$ polymer consisting of $76 \mathrm{nt}$ with a random sequence of $\mathrm{C}$ and $\mathrm{U}$, can not form a secondary structure and remains linear in structure predictions (not shown). The shSV40 and the CU58mer fragments were used to test for binding of the BCD homeodomain to randomly structured vs. unstructured RNA molecules. Since the shSV40 fragment is not a natural target of $B C D$, the structure of this fragment is referred to as random.

For RNA binding, 2.5 - 20 pmole homeodomain were incubated with 20 fmole BRE_257-319, 24 fmole shSV40 and 22 fmole CU58mer and the HD:RNA complexes separated in a native polyacrylamide gel (Fig. 3.5, lanes 1-15). A visible complex of the homeodomain and the BRE_257-319 fragment occurs at a concentration of 5 pmole of BCD homeodomain, whereas at similar concentrations the unrelated RNA fragments CU58mer and shSV40 are not bound by the homeodomain. In the EMSAs with the BRE_164-339 fragment, 40 pmole of BCD homeodomain were needed to produce a shift in mobility. With the BRE_257-319 fragment much less protein is required to produce shift in mobility. This shows that the shorter BRE_257-319 fragment is bound by the homeodomain with significantly higher affinity as the larger BRE_164-339 
fragment and therefore smaller amounts of probes were allowed in the binding reactions (20 fmole BRE_257-319 vs 300 fmole BRE_164-339).

I observed slower migrating complexes at increasing homeodomain concentrations in the gel, which were also noticeable in band-shift experiments with the BRE_164-339 fragment, (Fig. 3.5, lane 9 and 10, Fig. 3.6, lane 3). These super-shifts could be interpreted as RNA:HD complexes with either multiple homeodomain molecules bound to the RNA or with multiple homeodomain molecules associated in protein-protein-RNA interactions. Experiments at more stringent binding conditions (elevated $\mathrm{KCl}$ concentrations) showed that the super-shifts become inhibited with increasing salt concentrations (Fig. A1.4).

The BCD homeodomain also binds to CU58mer and shSV40 at high concentrations (with 20 pmole homeodomain, Fig. 3.5, lanes 5 and 15), suggesting that the bacterially expressed homeodomain has a general RNA-binding affinity ("stickiness"), which is independent of the RNA sequence or structure. Hence, the super-shift complexes observed with the cad 3'UTR fragments appearing at high homeodomain concentrations (40 pmole homeodomain with the BRE_164-339 fragment and 20 pmole homeodomain with the BRE_257-319 fragment) might reflect saturation of all specific binding sites and subsequent "sticky" binding to unspecific sites in the RNA fragment.

In order to test the specificity of the RNA-binding below homeodomain concentrations that lead to the non-specific binding just mentioned I performed competition experiments with low concentrations of BCD homeodomain (Fig. 3.6, lanes 4-15). 5 pmole of homeodomain were incubated with 20 fmole of BRE_257-319 and competed with cold BRE_257-319 (lanes 4-7), shSV40 (lanes 8-11) and CU58mer RNA (lanes 12-15). I find that the formation of the BRE_257-319:HD complex is inhibited about 2 times more effectively by cold BRE_257-319 than by cold shSV40 (compare lane 5 and 9) and does not seem to be severely inhibited by the cold CU58mer RNA (lanes 12-15). These results suggest that the homeodomain can bind directly and specifically to the BRE_257-319 fragment in vitro, but again, only with a relatively low binding affinity. Interestingly, the cold RNA competitor shSV40 is able to disrupt binding while the unstructured RNA molecule CU58mer is not. The ability to form secondary structures may therefore be necessary for competing BRE_257-319 binding. 


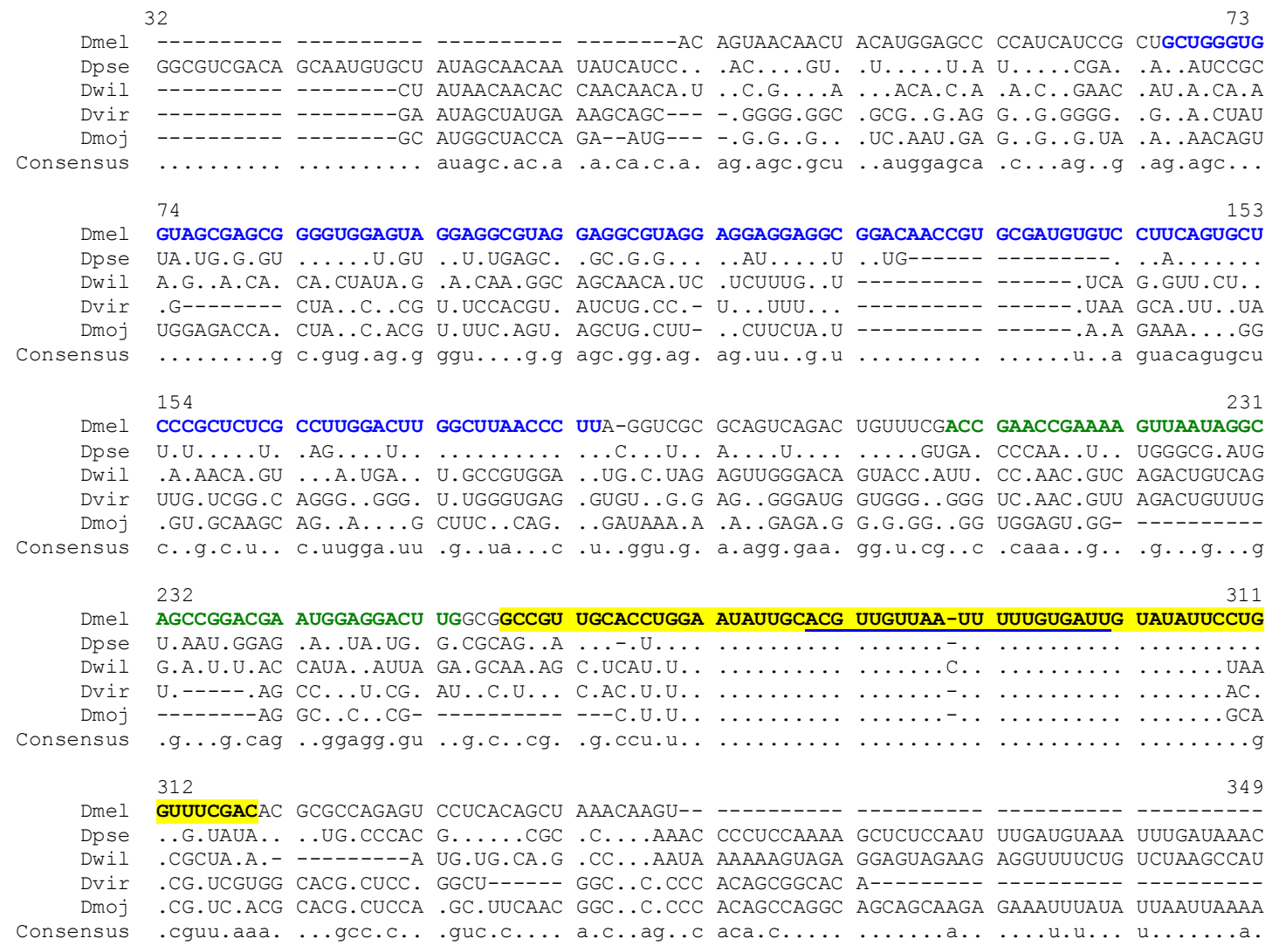

Fig. 3.4: Sequence alignment of cad 3'UTRs from different Drosophila species. The BBR_66-185 (indicated with bold, blue letters) and the BRE_210-253 (indicated with green, bold

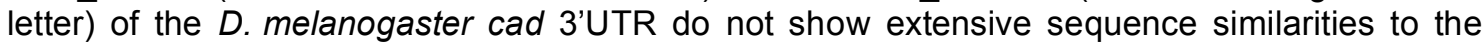
other Drosophila species. The BRE_257-319 fragment (indicated as yellow box) contains a 38 nt sequence region, which is present in all species (conserved nt are indicated with a point). Interestingly, this box overlaps with a predicted target site of miR-308 (underlined) in Drosophila (Brennecke et al., 2005; Stark et al., 2005). Identical nucleotide to the Dmel sequence are indicated by a dot. Numbering refers to the Dmel sequence, with 1 being the first nucleotide after the stop codon of the ORF. The full alignment can be seen in Fig. A2.1. Alignments were performed with Multalin program version 5.4.1. (Multiple sequence alignment with hierarchical clustering, CORPET, 1988, Nucl. Acids Res., 16 (22), 10881-10890) Dmel = D. melanogaster, Dpse $=D$. pseudooscura, Dwil $=D$. willistoni, Dvir $=D$. virillis, Dmoj $=D$. mojavensis. 

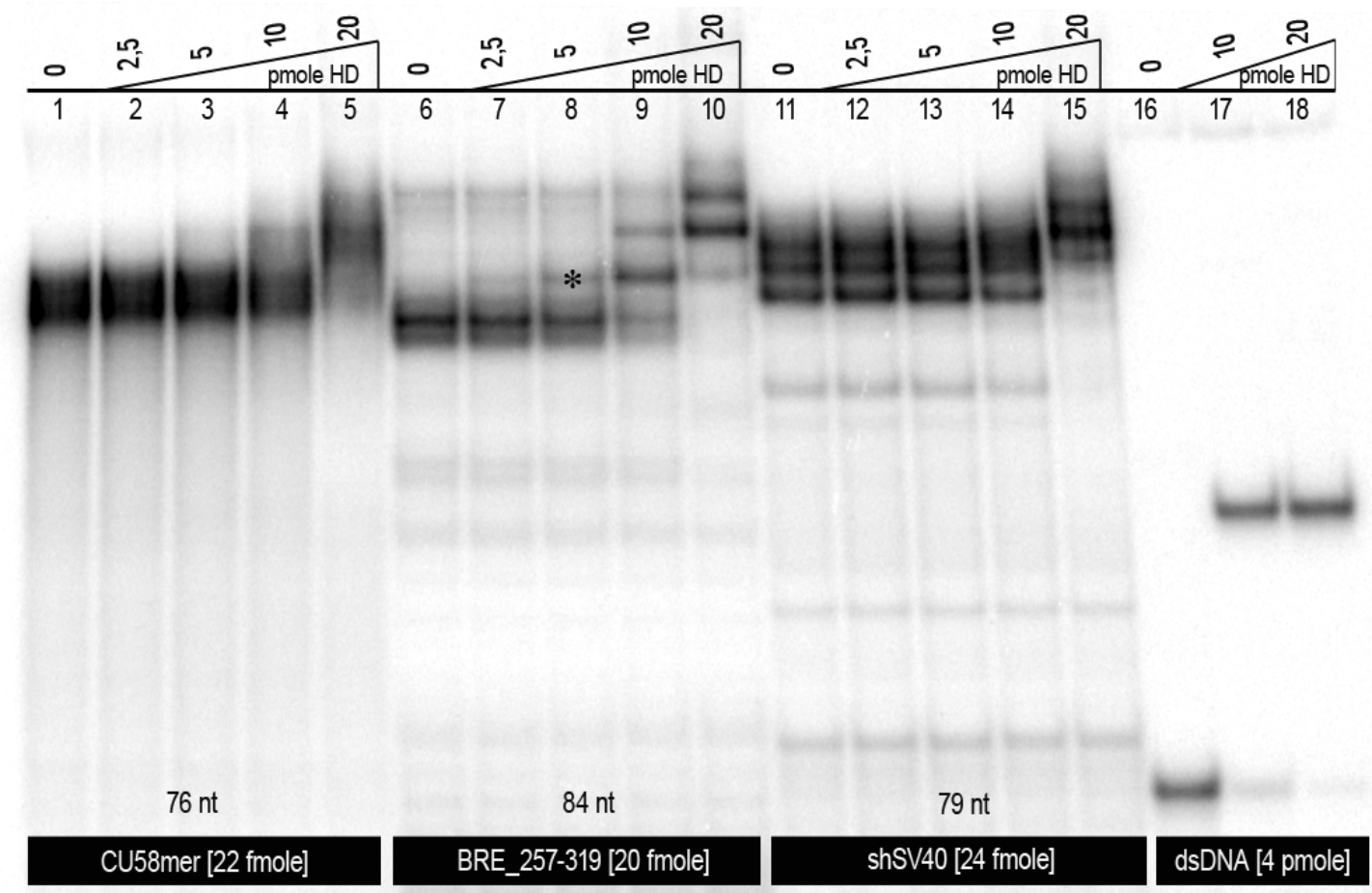

Fig. 3.5: Binding of BCD homeodomain to BRE_257-319 (lanes 5-10), to CU58mer RNA (lanes 1-5) and to shSV40 (lanes 11-15). The homeodomain binds to BRE_257-319 at a concentration of 5 pmole (lane 8, asterisk), but not to the unrelated RNA fragments (compare to lane 8 to 3 and 13). Lanes $16-17$ show DNA binding as a control for functionality of the homeodomain. 


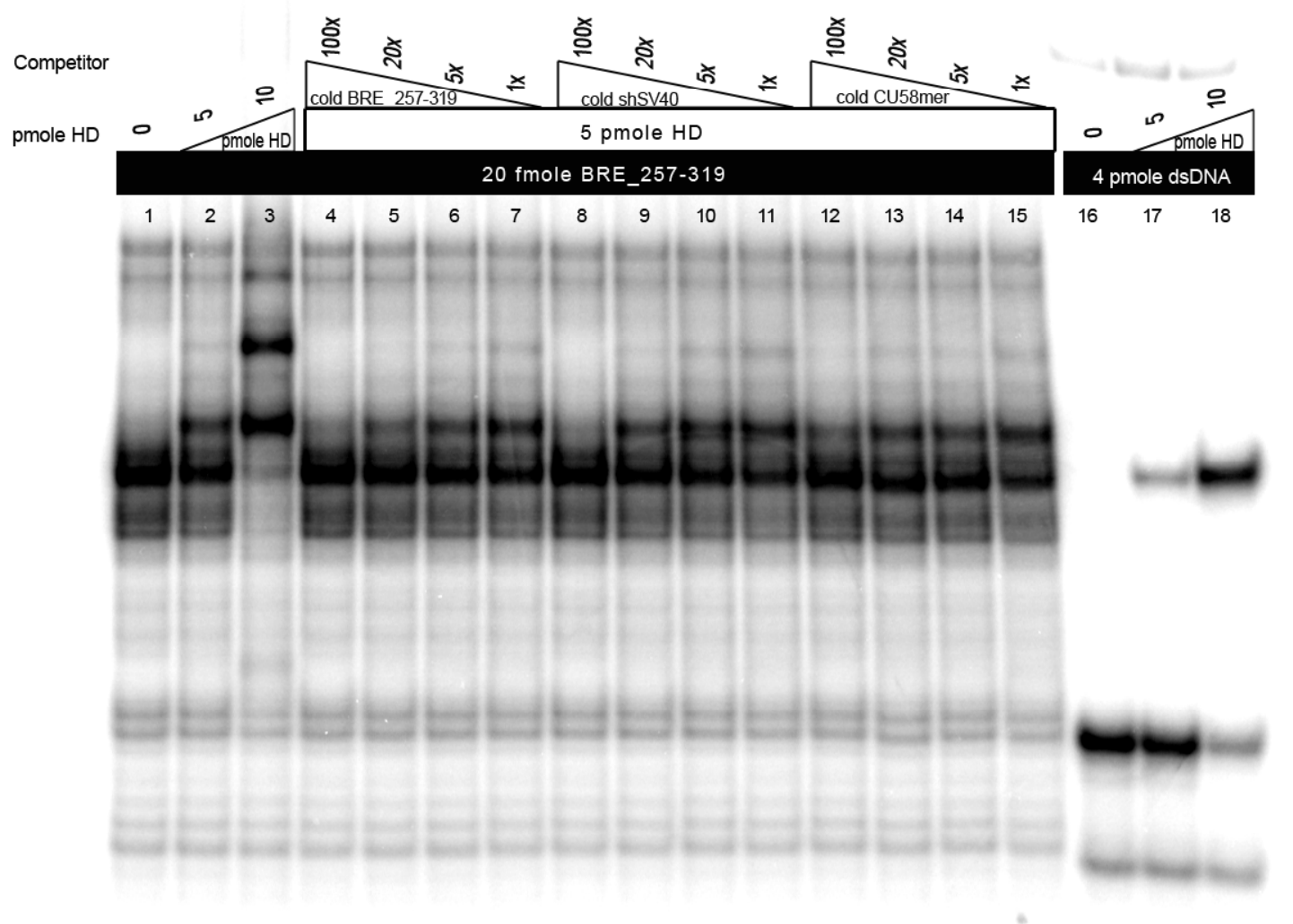

Fig. 3.6: Specificity of BCD homeodomain binding to BRE_257-319. The BRE_257-319 is shifted at a homeodomain concentration of 5 pmole (lane 2). Lanes 4-15 show competition of the BRE_257-319:HD complex with cold BRE_257-319, shSV40 and CU58mer. Binding of BRE_257-319 is competed slightly more efficiently by cold BRE_257-319 than cold shSV40 (compare lanes 5 and 9). The CU58mer RNA does not severely inhibit the formation of the BRE_257-319:HD complex (lanes 12-15). Lanes 16-18 show DNA binding, as a control for functionality of the homeodomain. 


\subsubsection{Identification of a Haematopota cad 3'UTR element that is bound by the BCD homeodomain}

An interesting candidate in the study of BCD binding elements is the cad 3' UTR homologue from the dipteran Haematopota. This fly expresses maternal cad (Hp'cad) whose transcripts are present in nurse cells and in the oocyte (Stauber et al., 2008), however a BCD homologue does not exist in Haematopota (Stauber et al., 2002) and sequence alignments of the Drosophila and Haematopota cad 3'UTRs do not show any obvious sequence similarities. The 3'UTR of Hp'cad (Hp3') consists of only 139 nt and is predicted to fold into a structure containing three stem-loops (H1 spanning nt $1-52$, H2 spanning nt 52 - 89 and H3 spanning nt 88 - 126 of the Hp'cad 3'UTR) predicted by the mfold RNA folding prediction program (Mathews et al., 1999; Zuker, 2003) (Fig.3.7, A). Fig. $3.7 \mathrm{~B}$ shows the predicted secondary structures of the three stem-loop structures of the Hp'cad 3'UTR individually. Of which only $\mathrm{H} 2$ is likely to exist, due to the low free energy value $\Delta G(\Delta G=26.99)$. Note that for fragments $H 1$ and $H 3$ each two secondary structures were predicted with $\Delta G$ values $>-10$, indicating that these structures might not exist individually, however they might be present in the full-length RNA molecule of the Hp'cad 3'UTR (Fig. A4.1). The BRE_257-319 fragment forms a stem-loop structure with $\Delta \mathrm{G}=-16.60$ (Fig. 3.7, C).

Interestingly, pairwise alignments of these distinct RNA structures using the RNAforester program (http://bibiserv.techfak.uni-bielefeld.de/rnaforester, (Höchsmann et al., 2003; Höchsmann et al., 2004)) revealed highest structure similarity between BRE_257-319 and $\mathrm{H} 2$, with structural similarity in the stem region (Fig. 3.7, D, marked in blue in the BRE_257-319 structure and in green in the H2 structure, Fig. A4.2).

In order to test whether the BCD homeodomain can bind to the Hp'cad 3'UTR, I performed band-shift experiments, using three different fragments (termed $\mathrm{H} 1, \mathrm{H} 2$ and H3). 30 pmole of each Hp'cad 3'UTR fragment were incubated with 2.5 - 7.5 pmole homeodomain (Fig. 3.8, lanes 9-20) and compared to binding of 30 pmole of BRE_257319 (Fig. 3.8, lanes 1-4). I observed that the BCD homeodomain can shift the $\mathrm{H} 2$ fragment (Fig. 3.8 lanes 15 and 16), but not H1, H3 or shSV40 (Fig. 3.8, shSV40 lanes 5 - 8, $\mathrm{H} 1$ lanes $9-12$ and $\mathrm{H} 3$ lanes $17-20)$. The observed shift of $\mathrm{H} 3$ in Fig. 3.8 could not be reproduced in an independent experiment. 
A Hp'cad 3'UTR: $\Delta \mathrm{G}=-28.99$
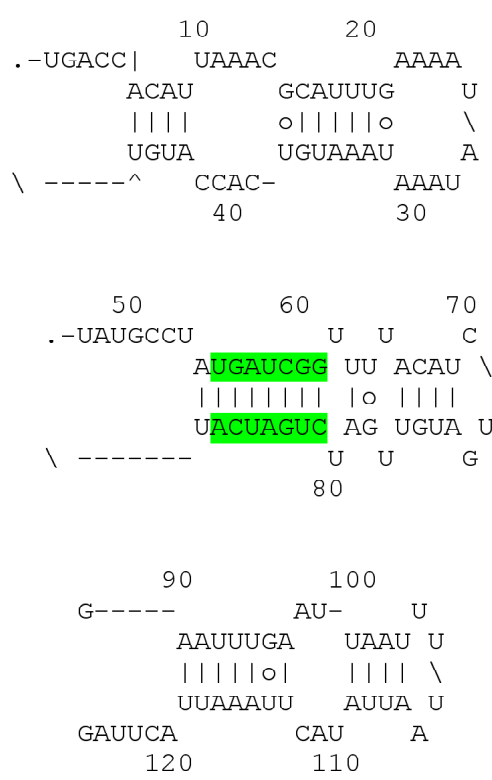

C BRE_257-319: $\Delta G=-16.60$

$$
\begin{array}{cccccc}
\text { CCC UUCC } & \text { U } & \multicolumn{2}{c}{30} \\
\text { CG } & \text { ACC } & \text { GGAAUAU } & \text { CACG } & \multicolumn{1}{c}{30} \\
|| & |||||||||| \mid & || \mid 0 & \text { UUCUU } \\
\text { GC } & \text { UGG } & \text { CCUUAUA } & \text { GUGU } & \text { A } \\
60 & & 50 & 40 & \text { UGUUUA }
\end{array}
$$

$\operatorname{CH}_{\mathrm{H} 2}^{\text {BRE_257-319 }}$

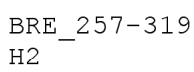

H2
B $H 1: \Delta G=-5.87$

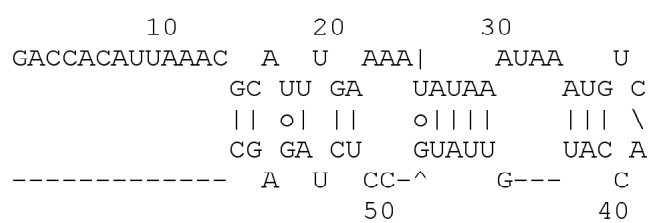

$H 2: \Delta G=-26.99$

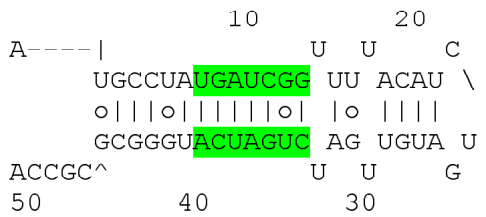

H3: $\Delta \mathrm{G}=-9.29$

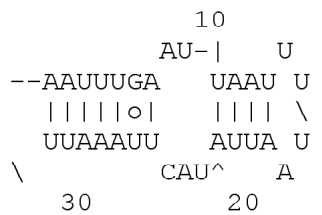

$$
\begin{gathered}
\text { ACUU GC } \\
\text { AGG । } \\
\text { I I | } \\
\text { UCC G } \\
---\quad \text { GC }
\end{gathered}
$$

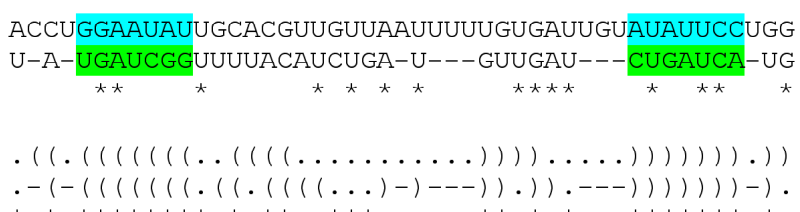

Fig. 3.7: Comparisons of secondary structures of the Hp'cad 3'UTR and BRE_257-319. (A) Secondary structures predicition of the Hp'cad 3'UTR and (B) the individual Hp'cad 3'UTR fragments $\mathrm{H} 1, \mathrm{H} 2$ and $\mathrm{H} 3$, numbers in parentheses indicate $\Delta \mathrm{G}$ at $25^{\circ} \mathrm{C}$. Note that $\mathrm{H} 2$ and BRE_257-319 show the highest $\Delta G$ values. (C) Secondary structure prediction of BRE_257-319 fragment. Secondary structure predictions were performed using the mfold program (version 2.3). (D) Secondary structure alignment of BRE_257-319 and $\mathrm{H} 2$ using the RNAforester program. The sequences of the stem-structures are indicated in blue (BRE_257-319) and green (H2). All RNAforester alignments can be seen in APPENDIX 4. 


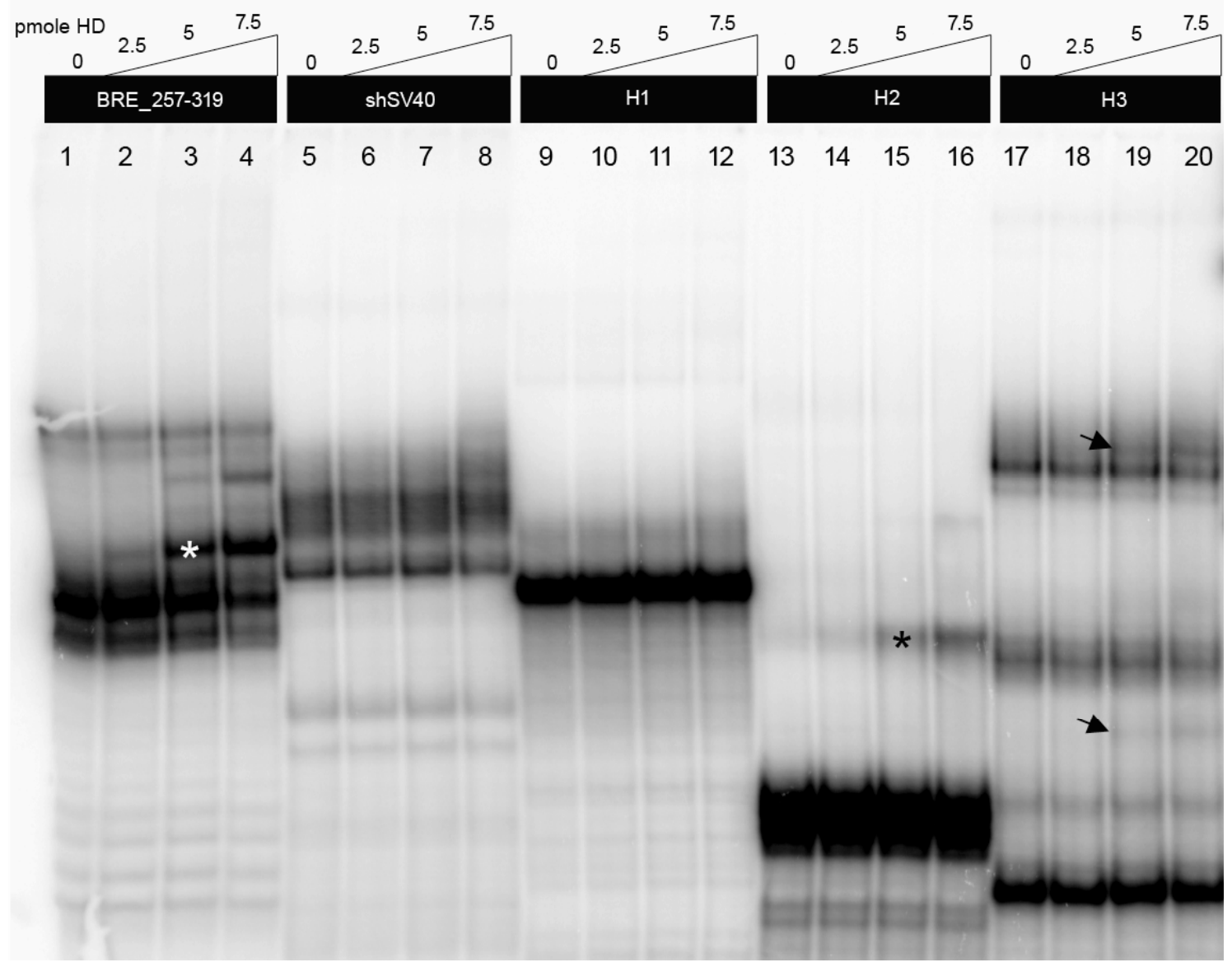

Fig. 3.8: Binding of the BCD homeodomain to Haematopota 3'UTR fragments H1, H2 and H3. Mobility shifts can be observed of the BRE_257-319 fragment (lane 3 and 4, white asterisk) and to a lesser extent of the $\mathrm{H} 2$ fragment (lane 15 and 16, black asterisk) at a homeodomain concentration of $5 \mathrm{nM}$, but not of shSV40 or $\mathrm{H} 1$ and $\mathrm{H} 2$ fragments. Shifted bands in lane 19 and 20 (arrows) could not be reproduced in an independent experiment. 


\subsubsection{Conclusions}

The bacterially expressed BCD homeodomain can bind to fragments of the cad 3'UTR (BRE_164-339 and BRE_257-319). However, competition experiments revealed that the binding occurs with low affinity, since these fragments are not more than 2-fold efficient as competitors than unrelated RNA fragments. The bacterially expressed BCD homeodomain used by Chan and Struhl (1997) was used in the presence of $1 \mu \mathrm{g} / \mu \mathrm{l}$ yeast tRNA and $2.5 \mu \mathrm{g} / \mu \mathrm{l}$ heparin. Both molecules are used as reagents to block unspecific binding of the RNA/DNA-binding BCD homeodomain. In comparison, the $B C D$ homeodomain discussed here shows a much lower tolerance for these reagents during the RNA-binding reaction. This could be the result of different protein purification methods used here and by Chan and Struhl (1997). I have used two different methods for the purification of the BCD homeodomain, which are dependent on the fusion peptide (His-tag and GST-tag purification). For both protein preparations methods I obtained similar results for the BCD homeodomain RNA-target affinity. The DNA/RNAbinding domain of the BCD homeodomain from both preparation methods seems to be intact, because DNA-binding and competition experiments revealed high DNA-binding specificity of these BCD homeodomains.

The unspecific RNA probe (Adh3') can not be directly bound by the BCD homeodomain, however its presence in a 5-100 fold excess to the specific probe inhibits the formation of the HD:BRE_164-339 complex extensively, albeit slightly less efficient than the specific competitor (BRE_164-339). This small difference in competition efficiency is most likely due to a somewhat higher binding specificity to the BRE_164-339 fragment than to the unrelated probe Adh3'. Nevertheless, there seems to be a discrepancy between the ability of the Adh3' probe to bind to the homeodomain and the ability to act as a competitor.

An explanation for this could be that Adh3' and the BRE_164-339 can interact with each other through imperfect or partial hybridizations, which interfere with BCD homeodomain binding. I noticed in an earlier EMSA experiment that higher running bands occur according to the addition of cold specific or unspecific competitor. These putative RNA-RNA-complexes were distinguishable according to their different sizes (not shown). In that particular EMSA and unlike in the here described method, the cold competitor was added to the labelled RNA probes prior to the addition of the protein. Hence, RNA-RNA-interactions between the labelled and unlabelled RNA molecules were given an advantage to occur and to form imperfect hybridized RNA-complexes. In 
combination with a low RNA-binding affinity of the BCD homeodomain, these RNARNA-hybrids may cause great interference to the formation of HD:BRE_164-339 complexes. In binding reactions, where the labelled BRE_164-339 probe was first added to the homeodomain and the competitors after homeodomain binding has occurred, the above mentioned RNA-RNA-interactions may be less likely to interfere. Generally, during the binding reaction the formation of equilibrium of the free RNA molecules, free protein and complexes of RNA and protein is depending of the RNAbinding affinity of the protein and the amount of specific RNA-binding sites in the RNA molecule. This process however is not static and protein and RNA constantly associate and disassociate. Therefore, besides the competitive binding of the cold RNA to the homeodomain, there may also have been competition by RNA-hybridizations between the competitor and the BRE_164-339. At the same time, the homeodomain may not be able to compete with these RNA-RNA-complexes, as the RNA molecules may have a stronger affinity towards each other than the BCD homeodomain to its targets in BRE_164-339. With smaller RNA probes, this effect should become smaller as the probability of cross-hybridizations becomes smaller. With the BRE_257-319 fragment I could achieve a slight improvement of BCD homeodomain RNA-affinity in the competition experiments (see below).

These putative RNA-RNA-interaction artefacts are not likely to occur in vivo, unless there is a role of RNA-RNA interactions of 3'UTR targets involved in RNA-binding of BCD. For example, dimerization of the bcd 3'UTR is essential for the bcd mRNA localization process during oogenesis and early embryogenesis. The bcd 3'UTR associates to form loop-loop interactions through intermolecular base-pairing, however the process of anterior localization requires additional factors that act within other regions of the bcd 3'UTR. The STAU target recognition is not dependent of the looploop interaction. (Ferrandon et al., 1997; Snee et al., 2005; Wagner et al., 2004; Wagner et al., 2001).

With the BRE_257-319 fragment, the affinity to the RNA-target becomes slightly increased, as much smaller amounts of BCD homeodomain are necessary to shift the BRE_257-319 probe. Furthermore, competition experiments with shSV40 and CU58mer as competitors showed that the BCD homeodomain is more affected by competitors that are structured such as shSV40, then by unstructured RNA such the $\mathrm{CU}$ polymer. This could be an indication for the general preference of the BCD homeodomain to interact with structured RNA molecules. Further indication for this can be taken from the binding experiments with the Hp'cad 3'UTR fragments $\mathrm{H} 1, \mathrm{H} 2$ and 
$\mathrm{H} 3$. From the three fragments only $\mathrm{H} 2$ is predicted to form a stable secondary structure, indicated by the $\Delta G$ value of -26.99 . In contrast, $H 1$ and $H 2$ have $\Delta G>-10$, which indicate that these fragments may exist as linear RNA molecules. Consequently, the $\mathrm{BCD}$ homeodomain can bind only to the $\mathrm{H} 2$ fragment and not to $\mathrm{H} 1$ or $\mathrm{H} 3$.

Binding of the homeodomain to the full Tribolium cad 3'UTR or to a shorter fragment termed Tc_245-307 (spanning nt 245-307) did not yield reproducible results (data not shown). The Tc_245-307 fragment was chosen as it showed a high degree of sequence similarity in sequence alignments with the BRE_257-319. This may be an indirect indication that the conserved BCD-binding element in the 3'UTR cad homologues may not depend on the primary sequence.

Taken together, the BCD homeodomain described here shows a low RNA-binding affinity, with a preference to bind to structured RNA molecules. Restrictions from the in vitro technique of competition experiments and EMSA may omit target specificity of the BCD homeodomain. It is possible that BCD binding to RNA targets behave more specific when tested in vivo. Analysis in vivo might provide better binding conditions and possibly presence of putative factors that are absent during the in vitro binding reaction. These factors could be additional domains of the BCD homeodomain or additional trans-acting factors, all of which may increase binding specificity.

To corroborate and further extent the results observed in the band-shift assays, the RNA fragments discussed here (except CU58mer and Adh3') have been analysed using the in vivo translational reporter assay discussed in chapter 3.2.

\subsection{Mapping Bicoid RNA-binding elements in vivo}

\subsubsection{The making of a fluorescent protein reporter assay in early Drosophila embryogenesis}

To define translational control elements in the 3'UTR of cad homologues in vivo, I aimed to establish a translational reporter assay using the $\varphi$ C 31 -integrase-mediated recombination system. The $\varphi \mathrm{C} 31$-integrase promotes site-specific integration of transgenes into a specific genomic locus. A major advantage of this system is 
avoidance of position effect due to different transgene insertion sites (Groth et al., 2003; Thorpe, 1998), allowing direct comparisons of transgene expression from different transgenic lines. By designing this translational reporter assay with a fluorescent marker gene containing a nuclear localisation signal, I aimed to observe in vivo the appearance of de novo translated protein from maternally provided reporter transcripts during early embryogenesis. I anticipated that if the fluorescent marker gene is followed by 3'UTR sequences with BCD-responsive elements (e.g. the cad 3'UTR) the translation of the marker protein along the anteroposterior axis would become inhibited in a BCD-dependent manner. In this reporter assay, the cad 3' UTR serves as a positive control for anterior repression by BCD, whereas the SV40 3'UTR (SV40) serves as a negative control, as it contains no known BCD-binding elements. Thereafter, the translational reporter assay could be used to determine whether 3'UTRs from cad homologues are regulated in a BCD-dependent manner.

Here I describe three strategies that I followed consecutively in the pursuit of a functional translational reporter assay. Translational reporter assays designed after strategy 1 and 2 did not sense anterior BCD-dependent translational repression of the reporter and therefore did not accomplish my initial requirements for a functional reporter assay (Fig. 3.9, $A$ and $B$, see 3.2.1.1 and 3.2.1.2). However, by supplementing the reporter of strategy 2 with UAS/GAL4 induced expression of BCD protein (strategy 2b) I was able to establish a reporter assay that faithfully reported BCD-dependent translational repression of reporter transcripts carrying the cad 3'UTR (see 3.2.1.3 and 3.2.2). Strategy $2 b$ was then used to analyse cad 3'UTRs of other insect species and additional experiments were carried out to investigate why the reporter in strategy 2 could not be translationally repressed by the endogenous BCD protein (see 3.2.1.4). Based on this investigation, I succeeded in the last months of my $\mathrm{PhD}$ in establishing a reporter that showed translational repression by anterior BCD in the embryos (strategy 3, see 3.2.1.4). 
A Strategy 1

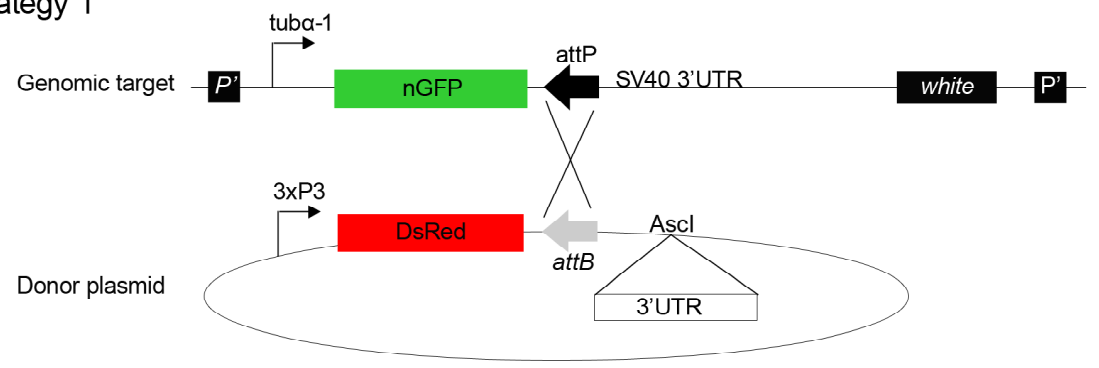

B Strategy 2

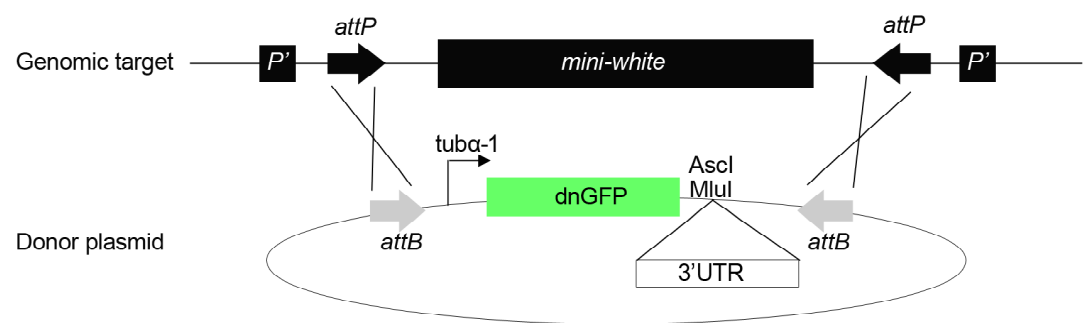

C Strategy 3

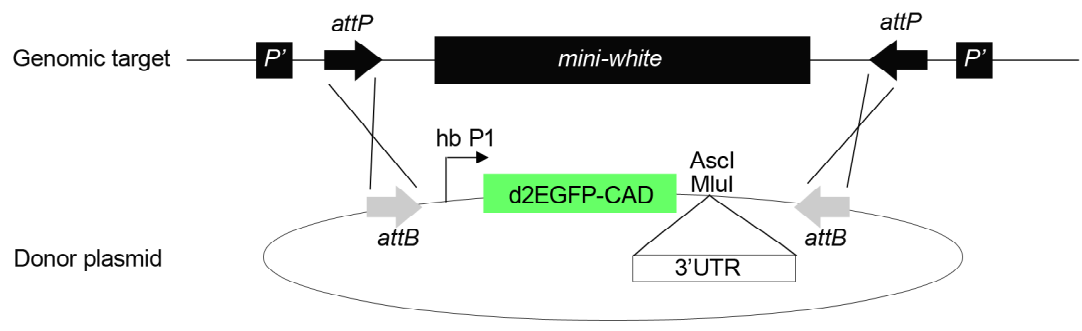

Fig. 3.9: Schematic overview of translational reporter assays. (A) Strategy 1: the genomic target sequence contains the tubulin $\alpha-1$ promoter (tub $\alpha-1$ ), an EGFP fusion with six Myc-tag repeats (6xMyc) and a nuclear localisation signal (NLS) (EGFP-6xMyc-NLS termed nGFP), the attP recombination site and the SV40 3'UTR. The donor plasmid contains the 3xP3-DsRed marker followed by attB and an Ascl site for cloning of 3'UTRs of interest. Upon an integration event the SV40 3'UTR will be replaced by the 3'UTR of interest. (B) Strategy 2: the genomic target consists of two inverted attP sites flanking the mini-white gene, which serves as a negative marker after the integration event has occured. The donor plasmid provides the reporter construct comprising of the tub $\alpha-1$ promoter, d2EGFP-6xMyc-NLS (termed dnGFP) and an Ascl or Mlul site for cloning of 3'UTRs of interest. d2EGFP is a EGFP variant with shorter half life time. Upon an integration event, the mini-white gene will be replaced by the reporter construct. Donor plasmid sequences will not be integrated. (C) Strategy 3: the genomic target is the same as in strategy $2 \mathrm{a}$, however the donor plasmid contains a modified reporter construct comprising of the maternal $h b$ P1 promoter (hb P1), a d2EGFP fusion with the Drosophila caudal protein (d2EGFP-CAD) and Ascl or Mlul sites for cloning of 3'UTR sequences of interest. 


\subsubsection{Strategy 1: Translational reporter assay using a single $\varphi$ C31-integrase recombination site}

The initial strategy was to establish $P$-element mediated transgenic lines, carrying the tubulin $\alpha-1$ promoter (tub $\alpha-1$ ) followed by an EGFP fusion with six Myc-tag repeats (6xMyc) and a nuclear localisation signal termed nGFP, an attP recombination site and the SV40 3'UTR. The donor plasmid contained the 3xP3-DsRed marker, an attB recognition site and a cloning site for inserting diverse 3'UTRs (Fig. 3.9, A). I established several independent transgenic lines containing the construct with the attP target site. I chose one transgenic line with an insertion on the second chromosome that was expressing uniform and moderate levels of nGFP in the syncytial embryo (termed pJBattPM44, Tab. 3.1). After integration of the donor plasmid into the attP target site of pJBattPM44, nGFP was no longer followed by the SV40 3'UTR, but by other 3'UTRs of interest. Table 3.1 summarizes the transgenic lines and the resulting genotypes that were generated using this approach. The introduction of 3'UTR carrying element(s) that are recognized by BCD (e.g. Drosophila cad 3'UTR) was expected to result in the formation of an anterior-to-posterior gradient of nGFP due to anterior translational repression. However, after integration of the donor plasmid pHAE-3xP3DsRed-attB-Dm3'-SV40 with the cad 3'UTR the anticipated anterior repression did not occur. Instead, the nGFP levels were reduced homogenously in the embryo and at all subsequent developmental stages (larvae, adults). The same was observed upon integration of donor plasmids carrying other 3'UTRs like the SV40 3'UTR or no 3'UTR at all (see Tab. 3.1), indicating that the integration of plasmid sequences, or the creation of an attL between nGFP and the 3'UTR of interest after the integration event, resulted in a general downregulation of nGFP levels. The reasons for this general nGFP downregulation remain unclear. Nevertheless, it was clear that this reporter assay is not suitable for the in vivo analysis of BCD-interacting 3'UTR elements. 
Tab. 3.1: Drosophila sensor lines generated by $\varphi C 31$-integrase mediated insertion into the pJBattPM44 locus on the second chromosome.

\begin{tabular}{|c|c|c|}
\hline Donor plasmid & Genotype after integration event & $\begin{array}{l}\text { Anterior } \\
\text { repression }\end{array}$ \\
\hline $\begin{array}{l}\text { pHAE-3xP3-DsRed-attB- } \\
\text { Dm3'-SV40 }\end{array}$ & $\begin{array}{l}\text { yw; P[w+, tub } \alpha-1-n G F P-a t t L-D m 3 \text { '_plasmid- } \\
\text { sequence_3xP3-DsRed-attR-SV40]/CyO }\end{array}$ & no \\
\hline $\begin{array}{l}\text { pHAE-3xP3-DsRed-attB- } \\
\text { Hp3'-SV40 }\end{array}$ & $\begin{array}{l}\text { yw; P[w+, tub } \alpha-1 \text {-nGFP-attL- } \\
\text { Hp3'_plasmid_sequence_3xP3-DsRed- } \\
\text { attR-SV40]/CyO }\end{array}$ & no \\
\hline $\begin{array}{l}\text { pHAE-3xP3-DsRed-attB- } \\
\text { Tc3'-SV40 }\end{array}$ & $\begin{array}{l}\text { yw; P[w+, tub } \alpha-1 \text {-nGFP-attL-Tc3'_plasmid- } \\
\text { sequence_3xP3-DsRed-attR-SV40]/CyO }\end{array}$ & no \\
\hline $\begin{array}{l}\text { pHAE-3xP3-DsRed-attB- } \\
\text { SV40 }\end{array}$ & $\begin{array}{l}\text { yw; P[w+, tub } \alpha-1-n G F P-a t t L-S V 40 \_p l a s m i d- \\
\text { sequence_3xP3-DsRed-attR-SV40]/CyO }\end{array}$ & no \\
\hline $\begin{array}{l}\text { pHAE-3xP3-DsRed-attB } \\
\text { (no inserted 3'UTR) }\end{array}$ & $\begin{array}{l}\text { yw; P[w+, tub } \alpha-1-n G F P-a t t L \_p l a s m i d- \\
\text { sequence_3xP3-DsRed-attR-SV40]/CyO }\end{array}$ & no \\
\hline
\end{tabular}

\subsubsection{Strategy 2: Translational reporter assay using the $\varphi$ C31-integrase mediated cassette exchange (RMCE)}

In order to avoid the integration of plasmid sequences, I decided to use the $\varphi \mathrm{C} 31$ integrase mediated cassette exchange (RMCE) system (Bateman, 2006). In this system a target cassette, which is inserted in the genome containing the mini-white gene flanked by two attP sites in inverted orientation, becomes exchanged with a donor cassette delivered by a plasmid carrying inverted attB sites flanking the desired construct. The advantage of this system is the exclusive integration of construct sequences into the target sites (Fig. 3.9, B). The recombination products attL will be flanking the construct sequence and absent in the transgenic transcripts and their 3'UTRs.

Several Drosophila lines transgenic for the RMCE target cassette are available; I chose line 38F1 (from here on called 38F), which is located on the second chromosome (Bateman, 2006). The donor plasmid used to deliver the sensor construct (Fig. 3.9, B), contained the tuba-1 promoter (T), a destabilized version of EGFP (d2EGFP, BD Biosciences Clontech) fused to 6xMyc and the NLS (termed dnGFP) and an Ascl site for cloning of 3'UTRs. Table 3.2 summarizes the transgenic lines created by $\varphi$ C31integrase-mediated RMCE events using this reporter construct carrying different 3'UTR 
sequences. Unexpectedly, embryos with the sensor construct carrying the cad 3'UTR showed no repression of dnGFP translation at the anterior pole (Fig. 3.10, A line 38FDm3', embryos on top row).

Seeking to explain why the embryos of the 38F-Dm3' sensor line were not showing the anticipated dnGFP gradient, I noticed that in ovaries of the females of these flies dnGFP protein is already present in nurse cell and oocyte nuclei (not shown). Maternally expressed mRNAs of embryonic patterning genes like caudal are supposed to be translationally inactive during oogenesis. The signals that mediate this are likely to reside in the 3'UTR of these messages (Tadros and Lipshitz, 2005). Unexpectedly, the 38F-Dm3' sensor does not seem to contain a signal(s) for translational repression during oogenesis and therefore the reporter protein is prematurely translated. This has some severe implications for the sensitivity of the reporter assay. In particular, if dnGFP protein is produced during oogenesis and dumped into the oocyte, then it will most likely perdure until embryogenesis begins and perhaps for an even longer period. Although the turnover rate of d2EGFP is only $2 \mathrm{~h}$ (in mammalian cell culture, BD Biosciences Clontech), the addition of the nuclear localisation signal could have significantly increased protein stability (G. Struhl, pers. comm.). In addition, the maturation time required for fluorescence of d2EGFP could limit the amount of visible, newly-produced EGFP in the embryo. Maternally produced fluorescent protein could thus mask the BCD-dependent gradient to such an extent that differences in fluorescence along the AP-axis are not detectable in the embryo.

Alternatively, the 38F-Dm3' sensor could be lacking signals that normally protect maternal cad transcripts from mRNA degradation. Insufficient amounts of maternal sensor transcripts in the embryo might make it more difficult to detect the effects of $B C D$ repression. These questions will be addressed in section 3.2.1.4. 
Tab. 3.2: Drosophila sensor lines generated by $\varphi$ C31-integrase-mediated RMCE. Integration of donor plasmids was performed into RMCE target cassette 38F1 on the second chromosome.

\begin{tabular}{|c|c|c|c|}
\hline $\begin{array}{l}\text { Name of donor } \\
\text { plasmid }\end{array}$ & $\begin{array}{l}\text { Name of } \\
\text { integrant line }\end{array}$ & $\begin{array}{l}\text { Genotype after integration } \\
\text { event }\end{array}$ & $\begin{array}{l}\text { Anterior } \\
\text { repression }\end{array}$ \\
\hline $\begin{array}{l}\text { piB-TdnGFP- } \\
\text { Dm3' }\end{array}$ & 38F-Dm3' & $\begin{array}{l}\text { yw; P[attR- tub } \alpha-1-d n G F P- \\
\text { Dm3'-attR] }\end{array}$ & no \\
\hline $\begin{array}{l}\text { piB-TdnGFP- } \\
\text { Dm3'mut }\end{array}$ & 38F-Dm3'mut & $\begin{array}{l}\text { yw; P[attR- tub } \alpha-1-d n G F P- \\
\text { Dm3'mut-attR] }\end{array}$ & no \\
\hline $\begin{array}{l}\text { piB-TdnGFP- } \\
\text { Hp3' }\end{array}$ & 38F-Hp3' & $\begin{array}{l}\text { yw; P[attR- tubo-1-dnGFP-Hp3'- } \\
\text { attR] }\end{array}$ & no \\
\hline $\begin{array}{l}\text { piB-TdnGFP- } \\
\text { Tc3' }\end{array}$ & 38F-Tc3' & $\begin{array}{l}\text { yw; P[attR- tub } \alpha-1-d n G F P-T c 3 '- \\
\text { attR] }\end{array}$ & no \\
\hline $\begin{array}{l}\text { piB-TdnGFP- } \\
\text { SV40 }\end{array}$ & 38F-SV40 & $\begin{array}{l}\text { yw; P[attR- tubo-1-dnGFP- } \\
\text { SV40-attR] }\end{array}$ & no \\
\hline $\begin{array}{l}\text { piB-TdnGFP- } \\
\text { BRE_257 }\end{array}$ & $\begin{array}{l}\text { 38F-BRE_257- } \\
319\end{array}$ & $\begin{array}{l}\text { yw; P[attR- tubo-1-EGFP- } \\
\text { 6xMyc-NLS-BRE_257-SV40- } \\
\text { attR] }\end{array}$ & no \\
\hline piB-TdnGFP-H1 & $38 \mathrm{~F}-\mathrm{H} 1$ & $\begin{array}{l}\text { yw; P[attR- tub } \alpha-1-E G F P- \\
6 x M y c-N L S-H 1-S V 40-a t t R]\end{array}$ & no \\
\hline piB-TdnGFP-H2 & $38 \mathrm{~F}-\mathrm{H} 2$ & $\begin{array}{l}\text { yw; P[attR- tub } \alpha-1-E G F P- \\
6 x M y c-N L S-H 2-S V 40-a t t R]\end{array}$ & no \\
\hline piB-TdnGFP-H3 & $38 \mathrm{~F}-\mathrm{H} 3$ & $\begin{array}{l}\text { yw; P[attR- tuba-1-EGFP- } \\
\text { 6xMyc-NLS-H3-SV40-attR] }\end{array}$ & no \\
\hline $\begin{array}{l}\text { piB-HdnGFP- } \\
\text { Dm3' }\end{array}$ & 38F-HDN-Dm3' & $\begin{array}{l}\text { yw; P[attR- hbP1-dnGFP-Dm3'- } \\
\text { attR] }\end{array}$ & no \\
\hline $\begin{array}{l}\text { piB-HdnGFP- } \\
\text { SV40 }\end{array}$ & $38 F-H D N-S V 40$ & $\begin{array}{l}\text { yw; P[attR- hbP1-dnGFP-SV40- } \\
\text { attR] }\end{array}$ & no \\
\hline $\begin{array}{l}\text { piB-HGFPCAD- } \\
\text { Dm3' }\end{array}$ & $\begin{array}{l}\text { 38F-HGFPCAD- } \\
\text { Dm3' }\end{array}$ & $\begin{array}{l}\text { yw; P[attR- hbP1-d2EGFP- } \\
\text { CAD-Dm3'-attR] }\end{array}$ & yes \\
\hline
\end{tabular}




\subsubsection{Strategy 2b: A translational reporter assay using RMCE in conjunction with BCD protein expressed using the UAS/GAL4 induction system}

Because of prematurely translated EGFP protein in the nurse cells and the oocyte of 38F-Dm3' females, I assumed that perdurance of prematurely produced EGFP protein during oogenesis omits the detection of BCD-mediated repression of the reporter in the embryo. I therefore addressed the question whether the reporter of 38F-Dm3' is susceptible to $B C D$ translational repression at the time when the reporter transcripts are being made, during oogenesis.

I employed the UAS/GAL4 induction system to ectopically express BCD protein during oogenesis and tested for the translational repression of the dnGFP reporter that carries the Dm'cad 3'UTR (38F-Dm3'). The ORF of BCD isoform G was fused at its C-terminus to 6xMyc repeats and inserted in the pUASp2 vector (Rørth, 1998) without bcd UTR sequences. Transgenic flies with the UAS-BCDG responder crossed to flies carrying the nos-GAL4:VP16 (Rørth, 1998) driver gave rise to female progeny that express BCD protein during oogenesis and deposit transgenic BCD protein and mRNA that is not subjected to anterior localisation, into the oocytes (two transgenic lines tested, UAS$\mathrm{BCDG}^{\mathrm{F} 4 \mathrm{M} 6}$ and $\mathrm{UAS}-\mathrm{BCDG}{ }^{\mathrm{F} 8 \mathrm{M} 1}$ ). The nuclear-localized transgenically expressed protein was detected by $\alpha$-Myc stainings (APPENDIX 3, Fig. A3.1). The function of transgenic $B C D$ as a transcriptional activator was tested by in situ hybridizations for the natural BCD target gene hunchback. Its activity as a translational repressor was tested by CAD antibody staining in embryos. Both ectopic activation of $h b$ and downregulation of CAD protein were observed (see 3.2.3.1, Fig. 3.13, D-F and Fig. 3.14, A and B).

Next, flies carrying both the sensor 38F-Dm3' and the UAS-BCDG were crossed to the nos-GAL4:VP16 line. dnGFP fluorescence intensities were measured in blastoderm stage embryos (nuclear division cycle 11) derived from females expressing the sensor 38F-Dm3', nos-GAL4:VP16 and either of the two UAS lines (maternal genotypes: UAS-

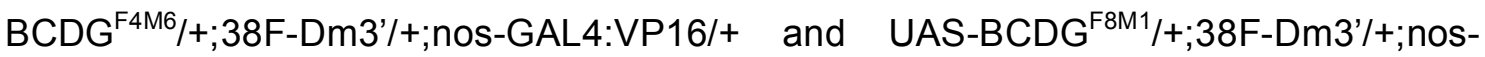
GAL4:VP16/+). For simplicity the embryos will be referred to with their mothers' genotype. The average dnGFP intensity of the sensor was compared in the absence $(n=22)$ and in the presence of the UAS responder $\left(n=30\right.$ for UAS-BCDG ${ }^{\text {F4M6 }}$ and $n=23$ for $U A S-B C D G^{F 8 M 1}$ ). Fig. $3.10 B$ shows that in the presence of UAS-BCDG the dnGFP intensities are reduced to about $40 \%\left(40 \% \pm 1.4\right.$ with UAS-BCDG ${ }^{\text {F4M6 }}$, $p=1.86 \times 10^{-10}$ and $41.8 \% \pm 1.7$ with UAS-BCDG ${ }^{\mathrm{F} 8 \mathrm{M} 1}, p=2.5 \times 10^{-10}$ ) of the dnGFP levels in flies carrying the sensor alone, indicating that the sensor expression is repressed by BCD. In contrast, dnGFP levels in embryos of the SV40 3'UTR sensor 
line (38F-SV40/+;nos-GAL4:VP16/+) did not change in the presence of ectopically expressed BCD (UAS-BCDG ${ }^{\mathrm{F} 4 \mathrm{M} 6} /+; 38 \mathrm{~F}-S V 40 /+;$ nos-GAL4:VP16/+, $p=0.24$, Fig. 3.10 C).

In summary, the translational reporter assay from strategy $2 a$ is not suitable for the detection of endogenous BCD-mediated repression. However, in combination with UAS/GAL4-induced ectopic expression of BCD protein, the 38F-Dm3' reporter expression reliably shows BCD-mediated repression of the cad 3'UTR. Thus, this reporter in conjunction with the UAS/GAL4 system, was applied for the analysis of BCD-dependent repression of cad 3'UTR homologues from different insect species (section 3.2.2 and Tab. 3.2). 
A

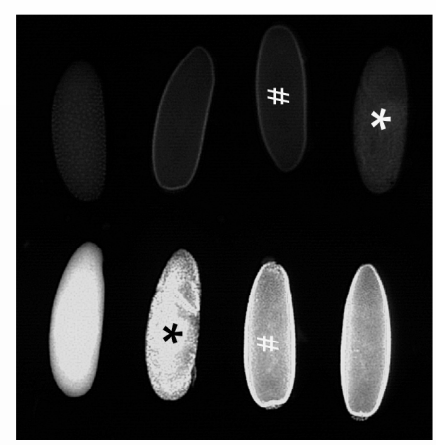

$\mathrm{B}$

38F-Dm3'

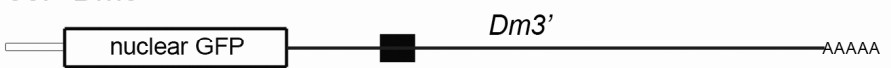

$38 \mathrm{~F}-\mathrm{SV} 40$

C
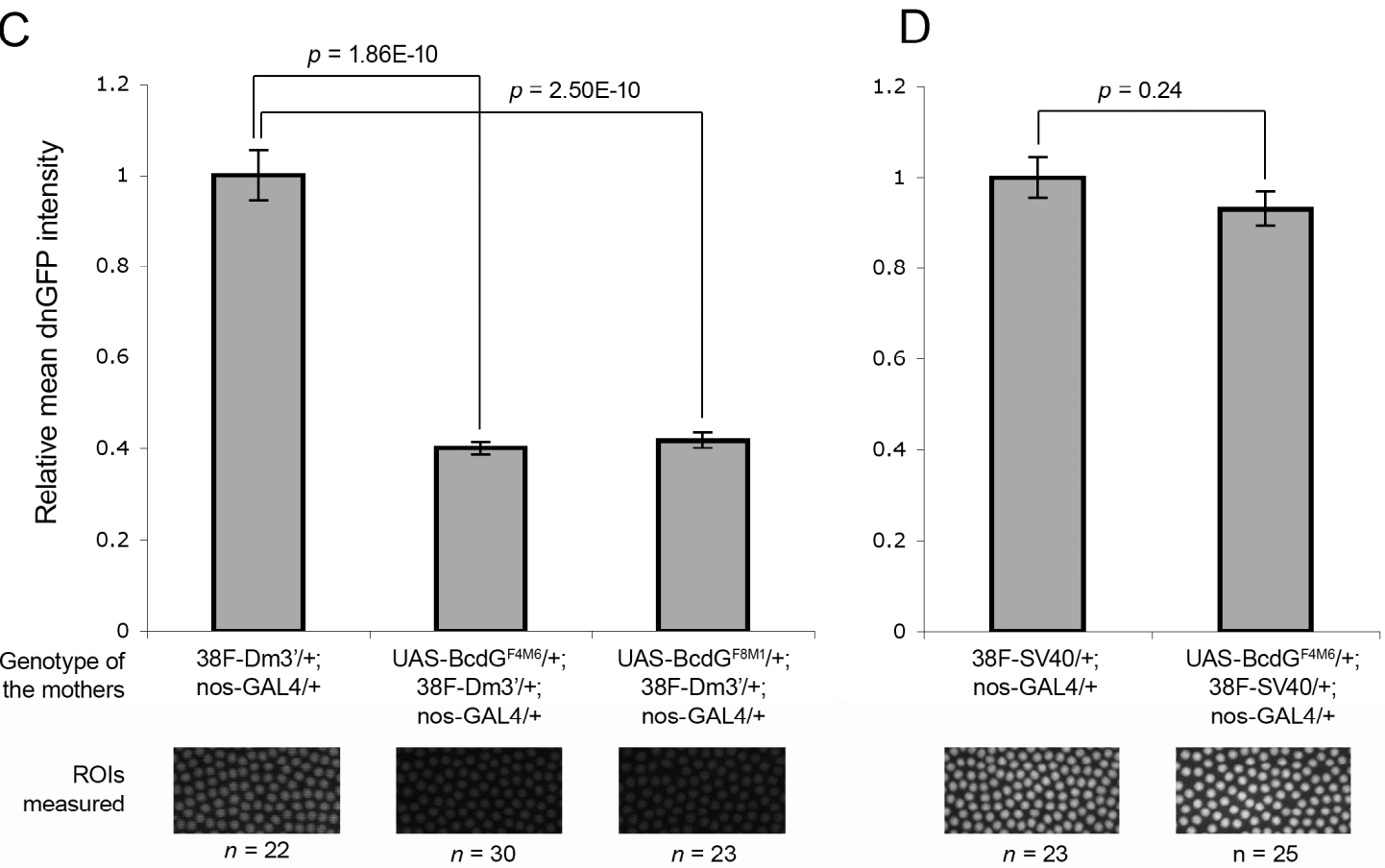

$n=23$

$n=25$

Fig. 3.10: Translational repression assay. (A) Comparison of dnGFP levels in transgenic Drosophila embryos of 38F-Dm3' (upper row) and 38F-SV40 (lower row). Note that not all embryos are at similar embryonic stages (similar stages are indicated by asterisks and dash sign), however overall dnGFP intensities are consistently lower in 38F-Dm3' embryos than in 38F-SV40 embryos. (B) Schematic overview of the reporter transcripts. The black box of the 38F-Dm3' transcript indicates the location of the BRE_257-319 fragment. (C) Relative dnGFP intensities measured in embryos of the 38F-Dm3' line in the absence (intensity level arbitrarily set to 1) and presence of two independent lines expressing UAS-BCDG (UAS-BCDG ${ }^{F 4 M 6}$ and UAS-BCDG ${ }^{\mathrm{FM} 1}$ ). Both UAS-BCDG lines induce a reduction of relative dnGFP levels by about $60 \%$. (D) The presence of UAS-BCDG ${ }^{\text {FM6 }}$ does not have an effect on the dnGFP intensity of $38 \mathrm{~F}-\mathrm{SV} 40$ line embryos. Bars represent relative mean values $\pm \mathrm{SE}$; statistical significance was analysed using Student's t-test $(p)$. GFP intensity values were taken from regions of interest (ROI) of identical size from the surface of blastoderm embryos at nuclear division cycle 11. 


\subsubsection{Strategy 3: Translational reporter assay using a d2EGFP-CAD fusion}

I conducted further investigations of cad 3'UTR homologues using the translational reporter assay developed in strategy $2 \mathrm{~b}$, using ectopically expressed $\mathrm{BCD}$ protein and the Drosophila cad 3'UTR as a positive control (see 3.2.1.3, 3.2.2). In the endeavour to corroborate this strategy and to be able to correctly interpret results generated with this translational reporter assay, I performed a set of control experiments. Furthermore, by investigating possible reasons for the premature presence of EGFP protein during oogenesis of the reporter from strategy 2, I aimed to generate a translational reporter that mediates translational repression via endogenous BCD.

Mlodzik et al. (1990) investigated the effect of heat-shock induced ectopic expression of cad and reported that anterior repression of cad translation was lost due to an mRNA overload in these embryos (Mlodzik et al., 1990). To test whether an overload of sensor transcripts might omit the detection of BCD-dependent repression I utilized the $h b$ maternal promoter, which is promoting expression at lower levels then the tuba-1. I created a reporter construct containing the maternal $h b \mathrm{P} 1$ promoter $(\mathrm{H})($ Wimmer et al., 2000), dnGFP and the cad 3'UTR (piB-H-dnGFP-Dm3'). The RMCE donor cassette with the reporter construct was inserted into the $38 \mathrm{~F}$ line, creating sensor line $38 \mathrm{~F}$ HDN-Dm3' (Tab. 3.2). In females of 38F-HDN-Dm3', I detected dnGFP protein in the nurse cells and the oocyte (not shown) and, although the overall dnGFP levels were much lower compared to the previous reporter (38F-Dm3'), there was no anterior repression detectable in the embryos (data not shown). Therefore, a sensor mRNA overloading effect is unlikely to have been the primary cause for lack of detectable anterior repression. Furthermore, the notion that premature degradation of the sensor mRNA could be preventing the formation of a protein gradient in the embryo could be ruled out as in situ hybridziations using an antisense probe of the reporter gene (d2EGFP) showed that sensor transcripts are present in the early embryo of 38F-HDNDm3' (data not shown).

Next, I addressed whether maternally inherited dnGFP protein together with longlasting GFP protein perdurance might be concealing the BCD-mediated repression of sensor transcripts in the embryo. Revising the literature, I noticed that in previous studies, the cad translational reporter assays utilized the reporter genes cad-lacZ, the $\mathrm{ftz}$ coding region or the Megaselia cad coding region for the detection of BCD-mediated repression (Dubnau and Struhl, 1996; Stauber et al., 2008). These reporters presumably had important properties, such as high protein turnover, which secures fast 
removal of maternal protein, or elements that prevent premature translation during oogenesis and that lack from my previous GFP reporters.

To address this question, I created a reporter construct containing the $h b$ maternal promoter (P1) and d2EGFP fused to the cad coding region, followed by the cad 3'UTR (Fig. 3.9, C). Due to the cad coding region, the d2EGFP-CAD fusion protein should localize to the nucleus, which facilitates the imaging of protein gradients in the syncytial blastoderm. Furthermore, I expected a high turnover rate similar to that of endogenous CAD protein, promoting fast removal of any maternally produced protein in the embryo. Dissected ovaries of flies containing the d2GFP-CAD sensor construct with the cad 3'UTR (38F-HGFPCAD-Dm3', schematic overview of the sensor transcript in Fig. 3.11, A) showed no GFP fluorescence (not shown), indicating the absence of translation of the sensor in the nurse cells and the oocyte, or a very fast degradation of translated protein. In the embryos, fluorescence of that reporter could be detected in an anteriorto-posterior gradient (Fig. 3.11, B), also detected by immunohistochemical stainings using $\alpha$-GFP antibody (Fig. 3.11, C). The mRNA of this reporter is distributed homogenously in the embryo, indicating that the protein gradient is generated through post-transcriptional regulation (Fig. 3.11, D).

Thus, the 38F-HGFPCAD-Dm3' reporter line faithfully reproduces the natural CAD gradient during early embryogenesis. These results indicate that the CAD ORF adds crucial properties to the fluorescent reporter, which were lacking in reporters 38F-Dm3' or 38F-HDN-Dm3' and which allow the formation of a translational repression gradient of the reporter protein in the 38F-HGFPCAD-Dm3' line. The reason why I could not detect a gradient in embryos of strategy 2 is most likely due to the perdurance of maternal dnGFP protein in the embryo or a lack of signal(s), residing in the CAD coding sequences that prevent translation of the sensor in the ovary. 

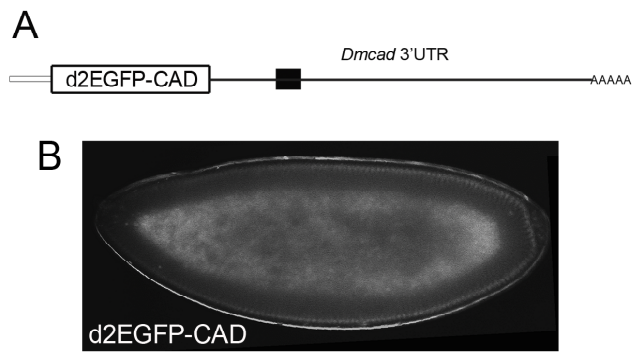

C
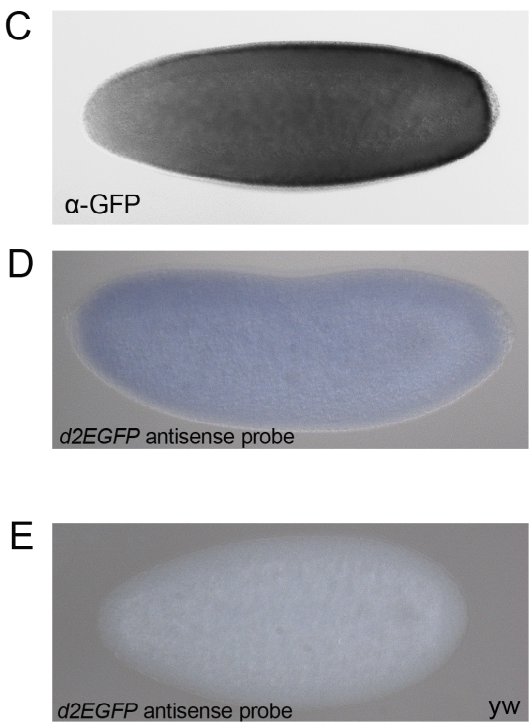

Fig. 3.11: Translational repression of a reporter carrying a d2GFP-CAD fusion and the Dm3'. (A) Schematic overview of the transgenic transcript. The ORF of the d2EGFP-CAD fusion protein is followed by the cad 3'UTR (Dm3'); the location of the BRE_257-319 fragment is indicated by a black box. (B) d2EGFP-CAD fluorescence in the blastoderm embryo of line 38 F-HGFPCAD-Dm3 ${ }^{\text {M52 }}$. The autofluorescence of the yolk is initially almost as strong as the d2EGFP-CAD fluorescence, however, it photobleaches very rapidly, whereas the fluorescence of the d2EGFP-CAD remains stable; these pictures were taken after 10-15 sec of photobleaching. Note the nuclear localization of the protein and the anterior lack of fluorescence as compared to the posterior end of the embryo. (C) $\alpha$-GFP immunohistochemical detection of the GFP-CAD reporter protein showing the asymmetric distribution of the reporter gene, with highest concentration at the posterior. (D) In situ hybridization using an antisense probe for d2EGFP-CAD mRNA. In contrast to the protein, the sensor transcripts are distributed homogenously throughout the embryo. (E) yw embryos are not stained using this probe. All embryos are oriented with anterior to the left and dorsal up. 


\subsubsection{Conclusions}

The Drosophila maternal genes have neatly orchestrated expression patterns and their proper spatiotemporal expression is of vital importance. Maternal gene expression is very dynamic, as BCD rapidly builds up an anterior-to-posterior protein gradient, necessary for activation of anterior gap genes and repression of translation of ubiquitously distributed cad mRNA. In fact, the establishment of the BCD gradient occurs so fast that current models trying to explain the formation of the gradient differ in their interpretations of how the gradient forms (Gregor et al., 2007a; Gregor et al., 2007b; Spirov et al., 2009). In concert with other maternal factors such as hunchback, nanos and torso, bcd activity provides key positional information for the patterning of segments along the AP-axis.

The time window of BCD activity ranges from 30 min to $2 \mathrm{~h}$ of development in which repression of cad mRNA occurs. Thus, for the establishment of a translational reporter assay that would detect BCD activity during early Drosophila embryogenesis, specific properties of the reporter protein were required. With the translational reporter assay (as in strategy 2), in which reporter mRNA and protein become deposited in the embryo, factors such as maturation time and lifetime of the reporter protein are of crucial importance for the detection of an emerging protein gradient in the embryo. In the reporter line 38F-Dm3' the detection of anterior BCD-dependent repression in the embryos is masked due to perdurance of maternal dnGFP protein, which is distributed ubiquitously in the embryo (strategy 2). By fusing the CAD coding sequence to d2EGFP, I changed the reporter protein properties such that the reporter with the cad 3'UTR became translationally repressed by endogenous BCD in the anterior of the embryo.

The cad 3'UTR faithfully reproduces BCD-dependent translational repression in the 38F-Dm3' line when the transcripts are exposed to ectopically expressed BCD protein during oogenesis (strategy $2 \mathrm{~b}$ ) or when the perdurance of the maternal reporter protein could be eliminated (strategy 3 ). 


\subsubsection{Identification of cad 3'UTR homologues that are translationally regulated by BCD}

\subsubsection{The BRE_257-319 fragment of the cad 3'UTR is mediating translational repression in the presence of $B C D$}

The BCD homeodomain shows weak binding affinity for the BRE_257-319 fragment in band shift experiments (see 3.1.2). I wanted to further this result, by investigating in vivo whether the translation of a sensor transcript bearing the BRE_257-319 fragment is regulated by $B C D$ (Fig. 3.12 A). Fig. $3.12 \mathrm{~B}$ shows that dnGFP levels of embryos derived from females expressing the sensor and $B C D$ isoform $G(y w, U A S-$ BCDG $^{\mathrm{F} 4 \mathrm{M} 6} /+; 38 \mathrm{~F}-\mathrm{BRE} \_257-319 /+;$ nos-GAL4:VP16) are reduced $(60.5 \% \pm 2.4$, $p=1.56 \times 10^{-12}$ ) compared to embryos whose mothers lack the UAS responder. This suggests that the sensor transcript bearing BRE_257-319 fragment is indeed recognized by $B C D$ and can mediate translational repression. In contrast, the sensor $38 \mathrm{~F}-\mathrm{SV} 40$ is not affected in the presence of BCD. It has to be noted that the only difference between transcripts of 38F-BRE_257-319 and 38F-SV40 is the presence of the BRE_257-319 fragment upstream of the SV40 3'UTR in 38F-BRE_257-319 (Fig. $3.12 \mathrm{~A})$. Interestingly, the dnGFP intensity is reduced in 38F-BRE_257-319 embryos to $59.2 \% \pm 1.7\left(p=3.49 \times 10^{-9}\right)$ as compared to the levels in 38F-SV40 (Fig. $3.12 \mathrm{~B}$ ). This result provides evidence for the existence of a BCD-independent cis-regulatory element(s) in the BRE_257-319 fragment and is concurring with the downregulation of dnGFP fluorescence observed in the sensor carrying the full Dm3' (38F-Dm3', see Fig. $3.10, A)$.

\subsubsection{The Haematopota cad 3'UTR mediates translational repression in the presence of BCD}

Sensor lines carrying the full Hp'cad 3'UTR (Hp3') or one of the three fragments $\mathrm{H} 1, \mathrm{H} 2$ or H3 of the Hp'cad 3'UTR followed by the SV40 3'UTR (see Tab. 3.2, Fig. 3.12 A) where crossed to the UAS-BCDG ${ }^{\mathrm{F} 4 \mathrm{M} 6}$ and the nos-GAL4:VP16 line. Fig. $3.12 \mathrm{C}$ shows the relative dnGFP intensities of the sensors measured in the absence (white bars) and presence (grey bars) of UAS-BCDG ${ }^{\mathrm{FM} 6}$ in embryos. The full Hp'cad 3'UTR mediates translational repression with sensor $38 \mathrm{~F}-\mathrm{Hp} 3$ ' and in the presence of $\mathrm{BCD}$ isoform $\mathrm{G}$ 
(UAS-BCDG ${ }^{\mathrm{F} 4 \mathrm{M} 6} /+; 38 \mathrm{~F}-\mathrm{Hp3}$ '/+;nos-GAL4) with dnGFP intensity of $80.8 \% \pm 2.4$ as compared to the dnGFP levels of the sensor alone $\left(p=2.97 \times 10^{-16}\right)$. In the EMSAs, I could show that only Hp'cad 3'UTR fragment $\mathrm{H} 2$ was bound by the BCD homeodomain (see 3.1.3).

In order to investigate which region of the Hp3'cad 3'UTR mediates BCD-dependent repression in the reporter assay, I tested the three fragments $H 1, H 2$ and $H 3$ individually. Only the sensor with fragment $\mathrm{H} 2$ mediates a significant reduction in dnGFP levels to $87 \% \pm 2.9$ in the presence of UAS-BCDG ${ }^{\mathrm{F} 4 \mathrm{M} 6}$ (maternal genotype: UAS-BCDG ${ }^{\mathrm{F} 4 \mathrm{M} 6} /+; 38 \mathrm{~F}-\mathrm{H} 2 /+;$ nos-GAL4/+) as compared to the sensors in the absence of the UAS responder $(p=0.001)$. These results confirm that BCD isoform $G$ can influence reporter activity via the $\mathrm{Hp}^{\prime}$ ' fragment $\mathrm{H} 2$ in vivo.

\subsubsection{The Tribolium cad 3'UTR fails to mediate BCD-dependent translational repression of the dnGFP sensor}

Wolff et al. (1998) showed that Tribolium cad cDNA, expressed in transgenic Drosophila embryos can be translationally repressed in a BCD-dependent manner. They concluded that the Tc'cad mRNA carries an element that is recognized by BCD. Because the BCD-binding element of the Drosophila cad mRNA is situated in the 3'UTR, it seemed likely that the element recognized by BCD in the Tc'cad mRNA might also be situated in its 3'UTR.

Sensor lines carrying the full Tc'cad 3'UTR (38F-Tc3', Tab. 3.2) where crossed to a line carrying UAS-BCDG ${ }^{\mathrm{F} 4 \mathrm{M} 6}$ and nos-GAL4:VP16, and the dnGFP intensities were measured in embryos (maternal genotype: UAS-BCDG ${ }^{\mathrm{F} 4 \mathrm{M} 6} /+; 38 \mathrm{~F}-\mathrm{Tc} 3{ }^{\prime} /+$ nosGAL4VP:16) (Fig. 3.12, C). The dnGFP intensities of sensor 38F-Tc3' showed no statistically significant difference in the presence or absence of BCD isoform G. This result indicates that the translational repression of the $T c^{\prime} c a d$ transcript observed previously (Wolff et al., 1998) is not mediated by its 3'UTR. 

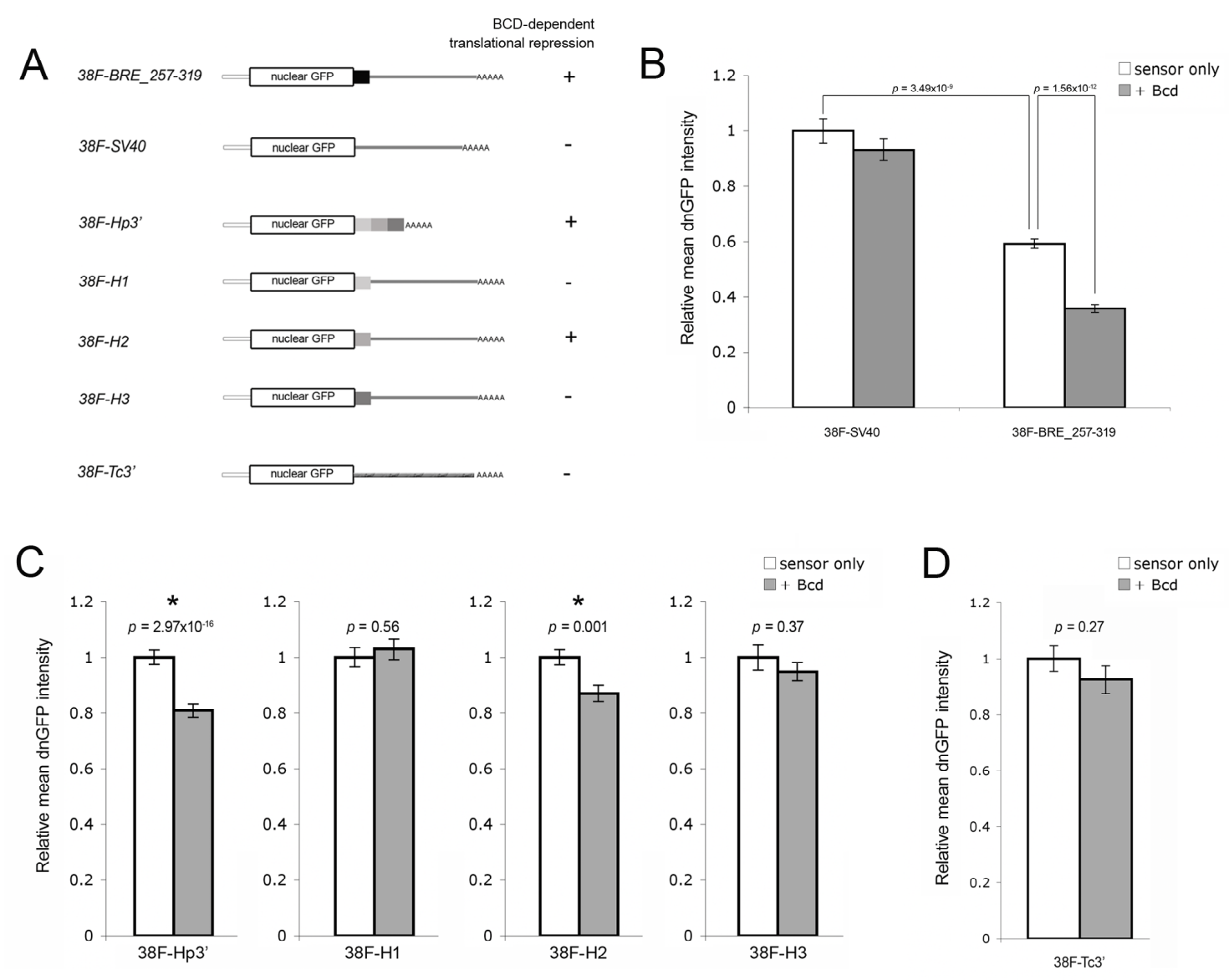

Fig. 3.12: Translational repression assay on BRE_257-319, Haematopota and Tribolium 3'UTRs. (A) Schematic overview of the reporter transcripts: 38F-BRE_257-319 transcript carries the BRE_257-319 fragment (black box) upstream of the SV403'UTR; 38F-SV40 carries the SV40 3'UTR; 38F-Hp3' carries the full Hp3'; 38F-H1, 38F-H2 and 38F-H3 carry the H1, H2 and H3 fragments upstream of the SV40 3'UTR; 38F-Tc3' carries the full Tc3'. (B) Average dnGFP intensities in the absence (white bars) or presence (grey bars) of UAS-BCDG ${ }^{\text {F4M6 }}$ in sensor lines 38F-SV40 and 38F-BRE_257-319. The relative fluorescence levels in 38FBRE_257-319 are about $40 \%$ lower than in 38F-SV40 $\left(p=3.49 \times 10^{-9}\right)$. In the presence of UASBCDG ${ }^{\text {F4M }}$ the relative dnGFP levels of 38F-BRE $257-319$ become reduced by about $40 \%(p=$ $1.50 \times 10^{-12}$ ) as compared to the sensor alone. (C) Average dnGFP intensities are reduced in the presence of UAS-BCDG ${ }^{\text {4M } 6}$ in sensors 38F-Hp3' and 38F-H2 $\left(p=2.97 \times 10^{-16}\right.$ and $p=0.001$, respectively). dnGFP levels in sensors $38 \mathrm{~F}-\mathrm{H} 1$ or $38 \mathrm{~F}-\mathrm{H} 3$ are unchanged in the presence or absence of UAS-BCDG ${ }^{\mathrm{F} 4 \mathrm{M} 6}$ (D) The dnGFP levels of sensor line 38F-Tc3' do not change in the presence of UAS-BCDG ${ }^{\mathrm{F} 4 \mathrm{MG}}$. Bars represent relative mean values $\pm \mathrm{SE}$; statistical significance was analysed using Student's t-test $(p)$. GFP intensity values were taken from regions of interest (ROI) of identically size from the surface of blastoderm embryos at nuclear division cycle $11(n=20-30)$. All carry nos-GAL:VP16.

\subsubsection{Conclusions}

Sensor $38 \mathrm{~F}-\mathrm{Dm} 3$ ' is reduced to $40 \% \pm 1.4 \mathrm{dnGFP}$ fluorescence in the presence of BCD isoform $\mathrm{G}$ as compared to the sensor alone. The sensor 38F-BRE_257-319 however is 
reduced to only $60 \% \pm 2.4$ dnGFP fluorescence levels, demonstrating that the fulllength cad 3'UTR is more effective than BRE_257-319 fragment alone. This may be due to the presence of additional sites in the cad 3'UTR that additively reduce translational repression. Alternatively, differences in the secondary structure present in full cad 3'UTR may be required for effective repression. The dnGFP levels of sensor $38 \mathrm{~F}-\mathrm{Hp} 3$ ' are reduced to $80.8 \% \pm 2.4$ and of sensor $38 \mathrm{~F}-\mathrm{H} 2$ to $87 \% \pm 2.9$ by $\mathrm{BCD}$ isoform $G$. These results suggest that the BRE_257-319 fragment may interact more effectively with $\mathrm{BCD}$ than the $\mathrm{H} 2$ fragment, leading to a stronger repression. Similarly, in EMSAs using the BCD homeodomain, I observed that complexes of the BCD homeodomain and the $\mathrm{H} 2$ fragment required much higher homeodomain concentrations as compared to complexes with the BRE_257-319 fragment, indicating a higher binding affinity of the homeodomain to BRE_257-319 than to H2. The dnGFP levels of the sensors containing the other Hp'cad 3'UTR fragments ( $\mathrm{H} 1$ and $\mathrm{H} 3)$ were not affected by BCD isoform G. Given that repression of a sensor with the full Hp'cad 3'UTR is stronger that with the $\mathrm{H} 2$ fragment alone, it is possible that in the context of the full Hp'cad 3'UTR the other regions flanking the $\mathrm{H} 2$ fragments can form a structure that allows stronger repression by BCD, either by providing additional BCD-binding elements or by stabilizing the BCD-binding element of the $\mathrm{H} 2$ fragment.

These results confirm the binding interactions revealed by EMSAs (see 3.1.2 and 3.1.3). The Tc'cad 3'UTR does not interact with BCD in the in vivo assay and RNAforester alignments with the Tc'cad 3'UTR did not detect any structure similarities with the BRE_257-319 fragment (APPENDIX 4). Thus, it cannot be excluded that the observed BCD-dependent translational repression of transgenic Tc'cad (Wolff et al., 1998) was mediated by an element situated in the 5' UTR or in the coding sequence. In fact, RNAforester alignment analysis of the predicted secondary structure of the Tc'cad 5'UTR+coding sequence and the BRE_257-319 fragment showed highest similarity to a region situated in coding sequence of Tc'cad (nt 451-485 with numbering starting with the first $n t$ in the sequence of NCBI ascession number AJ005421, APPENDIX 4). Similarly, the $\mathrm{H} 2$ fragment shows high structural similarity to the same fragment in the Tc'cad coding sequence. Judging from this predicted secondary structure analysis and from the BCD homeodomain binding results so far, I propose this fragment (spanning nt 451-485 of the Tc'cad cDNA) as a promising candidate for the cis-regulatory element in the Tc'cad mRNA that mediated previously observed BCD-dependent translational repression in Drosophila embryos. 


\subsection{Testing the role of different Bicoid isoforms during Drosophila early embryogenesis}

The bcd gene exists in 5 different splice isoforms (see Fig. 2.5). Current expression data for the morphogen bcd do not distinguish between different splice isoforms, because the probes used in these studies were usually obtained from the bcd cDNA that encodes the largest of the BCD protein isoforms (Berleth et al., 1988; Spirov et al., 2009). In fact, the generation of probes that would recognize individual isoforms is impossible, since none of the isoform transcripts contain sequences that are entirely unique to it.

However there is substantial evidence that each splicing product of bcd is present as maternal mRNA in the embryo: first, all isoform transcripts have been cloned from embryonic cDNA libraries and second, recent RNAseq data show that all exonic sequences of bcd are present in embryos (APPENDIX 5, Fig. A5.2).

Because it is not possible to deplete individual BCD isoforms, functional analyses are restricted solely to gain-of-function approaches. The five isoforms of BCD show some interesting features. Most notable are the absence of most known functional domains in isoform $\mathrm{A}$, and the lack of the 4EHP-binding domain in the putative proteins of isoforms $E$ and $F$. The latter are of particular interest regarding the mechanism of translational repression of cad mRNA, which was proposed to be mediated by the interaction of BCD with 4EHP.

In this study, the UAS/GAL4 system was employed to express each isoform of BCD in the context of the translational reporter system described above (see 3.2.1.3). The ectopic expression of each isoform individually will allow me to investigate their roles in the context of translational control of cad mRNA.

\subsection{1 hb transcriptional activation and CAD translational repression by different $B C D$ is oforms}

The ORFs of the five BCD isoforms were cloned from a Drosophila embryonic cDNA library into the pUASp2 construct and inserted into the genome through $P$-element mediated transgenesis. Maternal expression was induced ubiquitously in the embryo 
using the nos-GAL4:VP16 driver and embryos deriving from females expressing each UAS-BCD isoform by nos-GAL4:VP16 induction were collected for further analysis.

First, I analysed the ability of each isoform to ectopically activate expression of $h b$, which is a natural transcriptional target of BCD. Two transgenic lines of each UAS-BCD isoform were tested for their ability to activate the BCD target gene $h b$. In situ hybridizations were performed using an $h b$ ORF antisense probe and representative expression patterns are shown in Fig. 3.13.

In wt embryos (yw), hb mRNA are provided maternally and distributed homogenously in the early embryo (Fig. 3.13, A). hb becomes translationally regulated and forms an anterior domain (Fig. 3.13, B). All embryos with ectopically expressed BCD isoforms show normal maternal $h b$ expression in early embryos similar to wt (Fig. 3.13, compare A with $C, E, G, I, K)$. In pre-cellular embryos isoforms $G$ and $D$ have a strong activation effect leading to uniform $h b$ expression (Fig. 3.13, D and F) or to an expanded expression domain towards the posterior (not shown). Isoforms $E$ and $F$ induce a shift of the anterior expression domain towards the posterior in syncytial embryos, with the posterior boundary at $\sim 60 \% \mathrm{EL}$ (Fig. 3.13, $\mathrm{H}$ and J). Embryos with isoform A show wt expression of $h b$ transcripts (Fig. 3.13, K, L). In summary, all isoforms except isoform A induce dramatic shifts of the early anterior $h b$ expression domain towards the posterior albeit in slightly different fashions.

Next I investigated which isoforms can mediate translational repression of the endogenous cad mRNA, by using immunohistochemical stainings with an antibody against CAD. In blastoderm stage embryos (nuclear divison cycle 10-14) that derived from females expressing isoform G, D, E and F, cad mRNA translation was downregulated, as seen by a reduction of $\alpha$-CAD staining in comparison to stainings in wt embryos (Fig. 3.14). Embryos that derived from females expressing isoform A did not show any differences in $\alpha-C A D$ stainings as compared to wt (not shown). It has to be pointed out that isoform $\mathrm{E}$ and $\mathrm{F}$ do not possess the 4EHP-binding domain, which is thought to be necessary for d4EHP- and BCD-dependent translational repression of cad mRNA (Cho et al., 2005). To further substantiate these results, I analysed whether these isoforms can repress the translation of 38F-Dm3' sensor. 


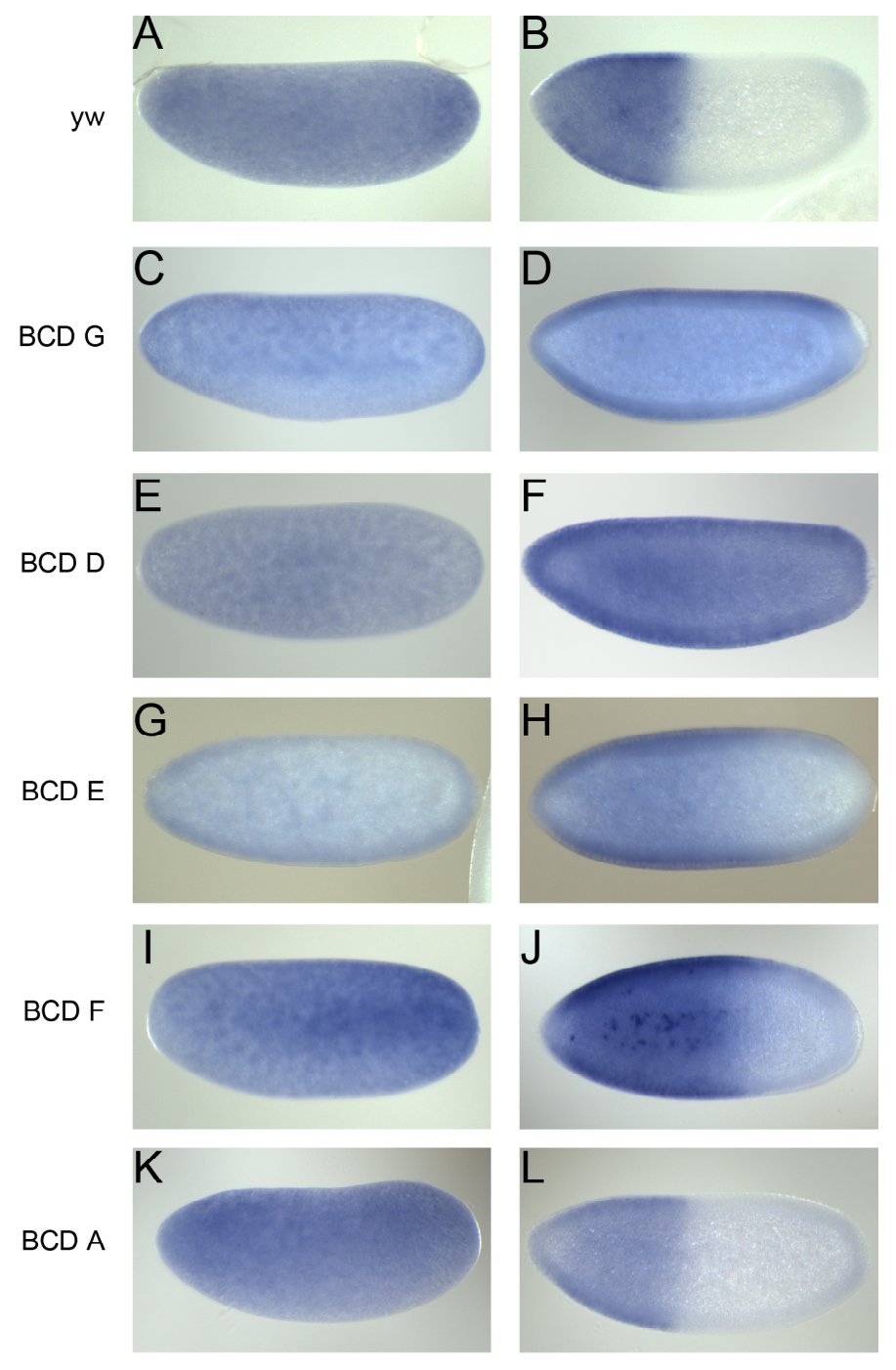

Fig. 3.13: $h b$ expression in the presence of differen $B C D$ isoforms. In situ hybridization using an antisense probe of the $h b$ ORF of wt embryos $(\mathbf{A}, \mathbf{B})$ and of embryos derived from females expressing isoform $G(\mathbf{C}, \mathbf{D})$, isoform $D(E, F)$, isoform $E(\mathbf{G}, \mathbf{H})$, isoform $F(\mathbf{I}, \mathbf{J})$ and isoform $A(K, L)$. Embryos in left column show pre-blastoderm embryos, embryos in the right column show pre-cellular blastoderm stages. Representative embryos are shown with anterior the left . 

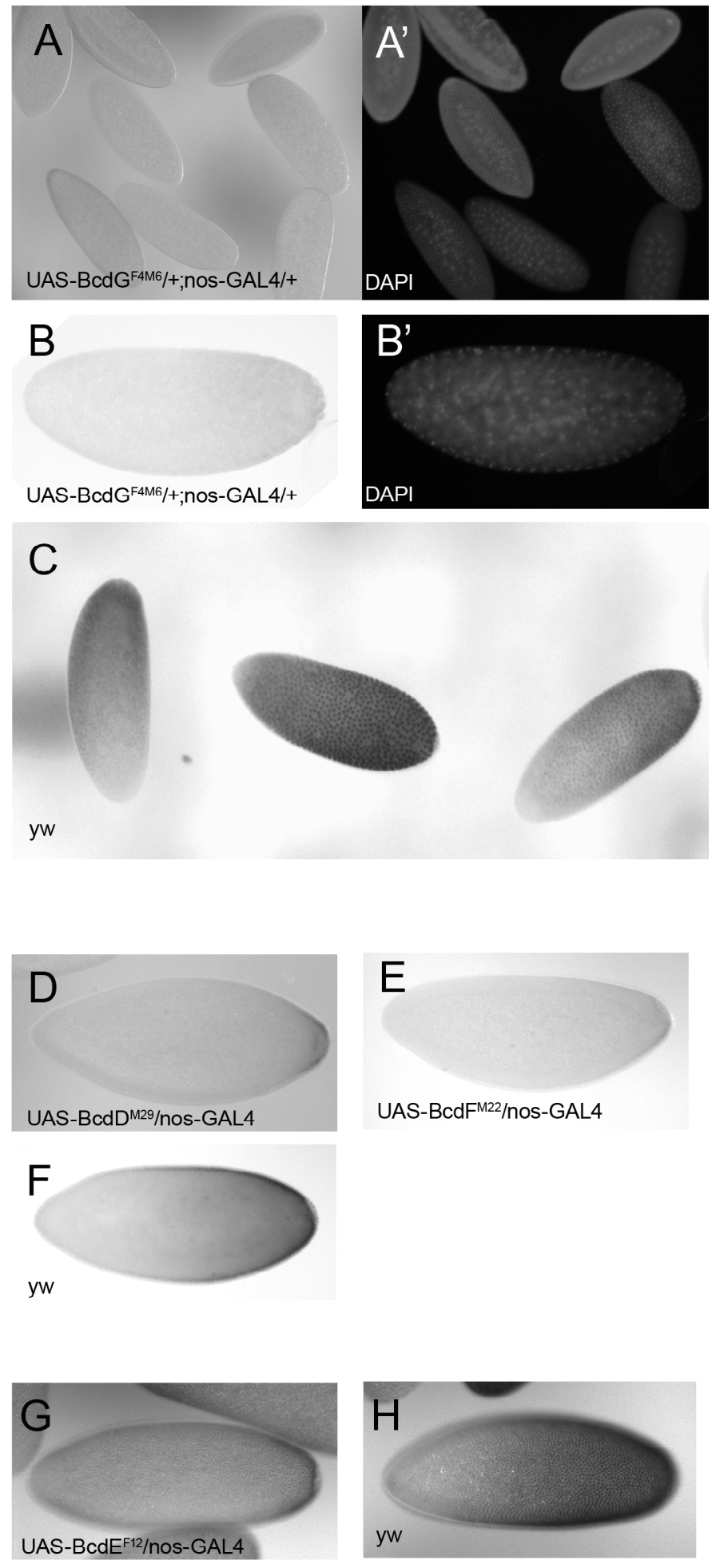

Fig. 3.14: cad translational repression in the presence of different $B C D$ isoforms. $C A D$ antibody stainings in blastoderm stage embryos derived from females expressing different BCD isoforms were performed on different days. yw embryos stained in parallel on the same day served as controls. Compare embryos derived from females expressing isoform $G(\mathbf{A}, \mathbf{B})$, isoforms $D$ and $F(D, E)$ and isoforms $E(\mathbf{G})$, to their respective controls $(\mathbf{C}, \mathbf{F}$ and $\mathbf{H})$. ( $\left.\mathbf{A}^{\prime}\right)$ and (B') show DAPI staining of the nuclei. 


\subsubsection{Translational repression of sensor 38F-Dm3' by different BCD isoforms}

Immunohistochemical-staining procedures can give variable results depending of the antibody and the strength of the staining and they may not be suitable to adequately quantify downregulation of CAD translation. Analysis of the dnGFP reporter can give more reproducible and quantitative results. The information output in form of fluorescence levels of the sensor representing the "wild-type" situation is much less variable.

I analysed the dnGFP levels of the sensor 38F-Dm3' in the presence of each BCD isoform in comparison to the sensor alone. Sensor line 38F-Dm3' was crossed to two transgenic UAS responder lines of every $B C D$ isoform in the presence of nosGAL4:VP16. The dnGFP intensities were measured in embryos deriving from females expressing the sensor and the respective BCD isoform (see Fig. 3.15 for maternal genotypes). Fig. 3.15 shows that the average dnGFP intensity of the sensor is reduced to about $40 \%$ in the presence of BCD isoform G $\left(40 \% \pm 1.4\right.$ with UAS-BCDG ${ }^{\mathrm{F} 4 \mathrm{M} 6}$ and $41.8 \pm 1.7$ with UAS-BCDG $\left.{ }^{\mathrm{F} 8 \mathrm{M} 1}\right)$, isoform $\mathrm{E}\left(41.5 \% \pm 1.6\right.$ with UAS-BCDE ${ }^{\mathrm{F} 12}$ and $38.3 \% \pm 1.4$ with UAS-BCDE $\left.{ }^{\mathrm{F} 79}\right)$ and isoform $\mathrm{F}\left(41.7 \% \pm 0.8\right.$ with UAS-BCDF $^{\mathrm{F9M} 3}$ and $45.5 \% \pm 0.9$ with $\mathrm{UAS}^{\mathrm{B}} \mathrm{BCDF} \mathrm{F}^{\mathrm{M} 22}$ ). $\mathrm{BCD}$ isoform $\mathrm{D}$ gives a reduction in fluorescence levels to about $30 \%$ of the initial dnGFP levels $\left(27.1 \% \pm 1.3\right.$ with UAS-BCDD ${ }^{\mathrm{F} 15}$ and $31.5 \% \pm 1.3$ with $U A S-B C D D^{M 29}$ ). Isoform $A$ does not seem to have an effect on the fluorescence levels of the sensor.

Taken together, these results confirm the conclusions drawn from immunohistochemical stainings of CAD protein in embryos with ectopic expression the $B C D$ isoforms. Isoforms $G, D, E$ and $F$ can repress translation of sensor transcript carrying the cad 3'UTR. 


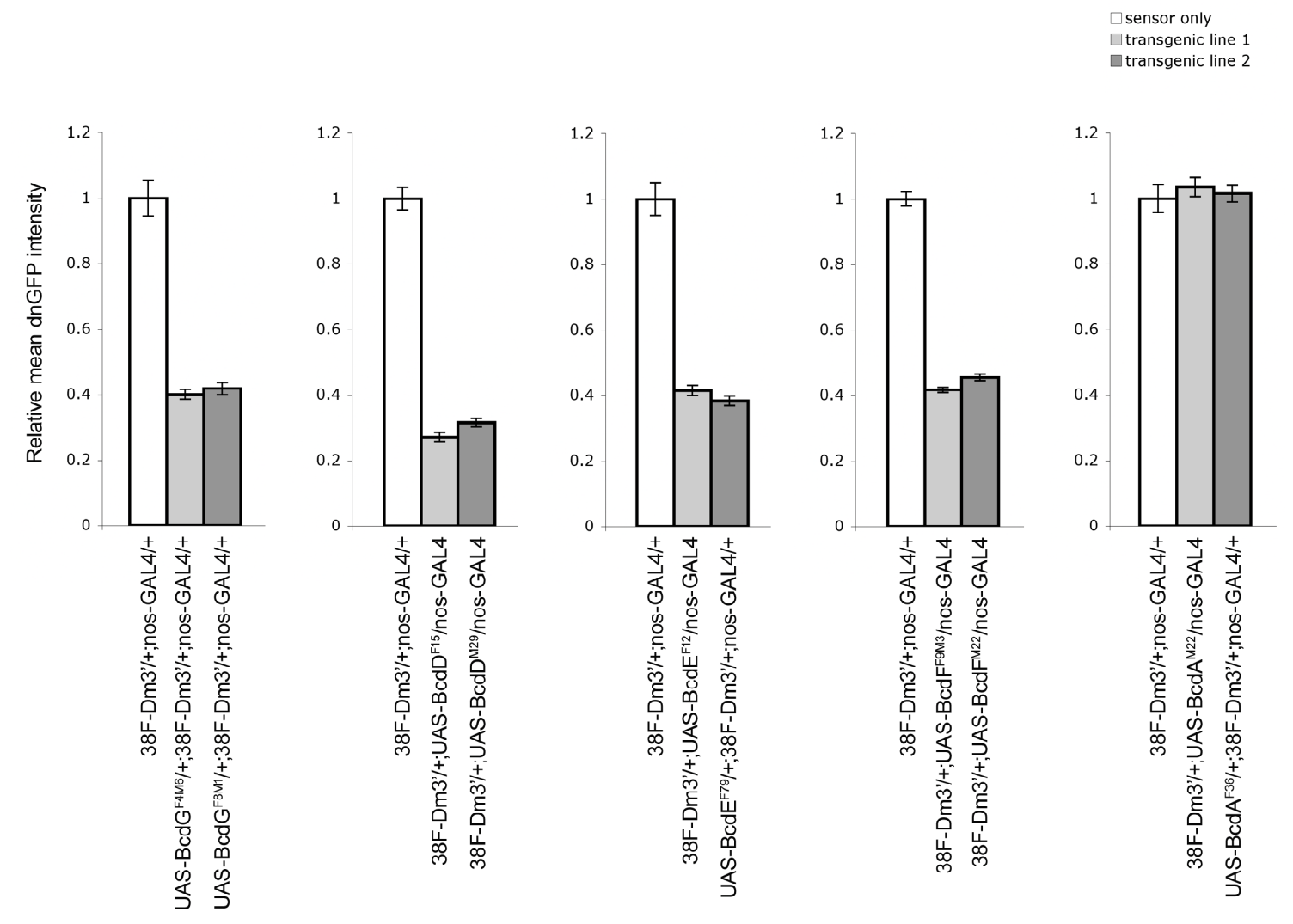

Fig. 3.15: Translational repression effect of different BCD isoforms on the 38F-Dm3' sensor. Average dnGFP intensities were measured in embryos deriving from females expressing the sensor 38F-Dm3' in the absence (white bars) or in the presence of the BCD isoforms (grey shaded bars, two lines analysed). Isoforms G, D, E and F give significant reduction of dnGFP levels of the sensor; isoform $A$ does not seem to have an effect on dnGFP fluorescence levels.

\subsubsection{Conclusions}

As previously described, the binding of d4EHP to both BCD and the cap-structure is critical for translational repression of cad mRNA (Cho et al., 2005). In hypomorphic mutants of d4EHP (d4EHP ${ }^{C P 53}$ ), cad mRNA becomes ectopically expressed in the anterior, however only about $50 \%$ of the progeny deriving from homozygous $d 4 E H P^{C P 53}$ females did hatch, the other $50 \%$ died due to severe patterning defects mostly affecting anterior segmentation (Cho et al., 2005). This hypomorph was produced through $P$ element excision of the first exon and part of the first intron of the d4EHP gene, creating transcripts with an alternative ATG start codon. These transcripts give rise to truncated d4EHP protein detecable by western blots. The presence of a partial CAD gradient and hatching rate of $50 \%$ of these hypomorphic mutants was explained 
stipulating a residual activity of d4EHP in these embryos (Cho et al., 2005). However it is not clear whether this hypomorphic d4EHP protein is active.

My results from the gain-of-function study of the BCD isoforms, suggests an alternative explanation. I show that even in the absence of a d4EHP-binding domain, isoform $\mathrm{E}$ or $F$ can still the translationally repress transcripts bearing the cad 3'UTR. The ectopic activation of $h b$ transcription illustrates that these two protein isoforms are not impaired in their function as transcriptional activators and that thus the homeodomain, which is the DNA/RNA-binding domain, is functional. It is therefore possible that the CAD translational repression observed in $d 4 E H P^{C P 53}$ embryos was not a result of residual d4EHP activity, but mediated through an alternative mechanism, which functions independently of d4EHP.

CAD translational repression could be result of cooperative action of endogenous $B C D$ protein (bearing the d4EHP-binding domain) with the ectopically expressed BCD isoforms. However this is not the case in the analysis with the translational reporter assay. As previously noted, the sensor reveals dnGFP protein produced during oogenesis, and its regulation by $B C D$ isoforms expressed during that stage using the nos-GAL4:VP16 driver.

So far, it is not known whether the individual BCD isoforms actually exist as proteins in the embryo and it would be interesting to further address this question in the future. The existence of different BCD isoforms or BCD-modifications could have important implications for the formation of the BCD activity gradient in the early embryo, an issue that has not been taken into account in most models of BCD action (Grimm et al., 2010). For instance, the $B C D$ isoform pairs $G / D$ and $E / F$ differ from each other in a short peptide sequence (DVFPS), which is present only in isoform $G$ and $E$, while isoforms $E$ and $F$ are missing a large part of the $\mathrm{N}$-terminus, which is present in isoforms $G$ and $D$. These peptide sequences could be post-translationally modified or mediate protein-protein interactions, which influence the activity of the protein. Different isoforms might also interact and exert different functions cooperatively. 


\subsection{Hints for alternative mechanisms of BCD-mediated translational repression}

The cad 3'UTR contains predicted target sites for the miRNAs miR-308 (target sequence nt 299-316) and miR-305 (target sequence nt 534-545) (Brennecke et al., 2005; Stark et al., 2005). The miR-308 binding target lies within the sequence that is highly conserved among different Drosophila species of the BRE_257-319 fragment (Fig. 3.16). The predicted secondary structure of the BRE_257-319 fragment contains a putative helical region of seven base pairs (termed stem-A), a short stem of three Watson-Crick base pairs and one $G \cdot U$ wobble pair (termed stem-B) and a hairpin-loop (Fig. 3.16). This structure is consistently predicted within the sensor transcripts, either within the full Dm3' (in 38F-Dm3') or when BRE_257-319 is followed directly by the SV40 3'UTR (in 38F-BRE_257-319) (not shown). The miR-308 target site, which is not overlapping with stem-A, constitutes most of stem-B and the hairpin loop (indicated in yellow, Fig. 3.16). Similarly, the H2 fragment of the Hp3' forms a stem-loop structure (termed H2-stem-A), which shows some structural similarity to stem-A of BRE_257-319 in alignments of the predicted secondary structures using the RNAforester program (see Fig. 3.7). The $\mathrm{H} 2$ fragment, however, does not contain any predicted Drosophila miRNA target sites. Thus, when tested in Drosophila, the $\mathrm{H} 2$ fragment is an RNA fragment that is regulated most likely independently of miRNA function.

So far there are two indications that point towards independent mechanisms for translational control of cad. First, the putative miRNA mediated repression of sensors carrying Drosophila cad 3'UTR (38F-Dm3', see 3.2.1.3) or BRE_257-319 (38FBRE_257-319, see 3.2.2.1) and second, d4EHP-independent translational repression of sensor 38F-Dm3' by BCD isoform $E$ or $F$ (see 3.3.2). In this part, I am asking whether miR-308 is likely to be involved in translational control of cad and whether it might interact with BCD to mediate translational control. Can we learn something about the d4EHP-independent mechanism of translational repression by studying the effects of miR-308? Can the $\mathrm{H} 2$ fragment, which lacks putative Drosophila miRNA binding sites be repressed by $B C D$ isoform $F$ (which lacks 4EHP-binding domain)? 

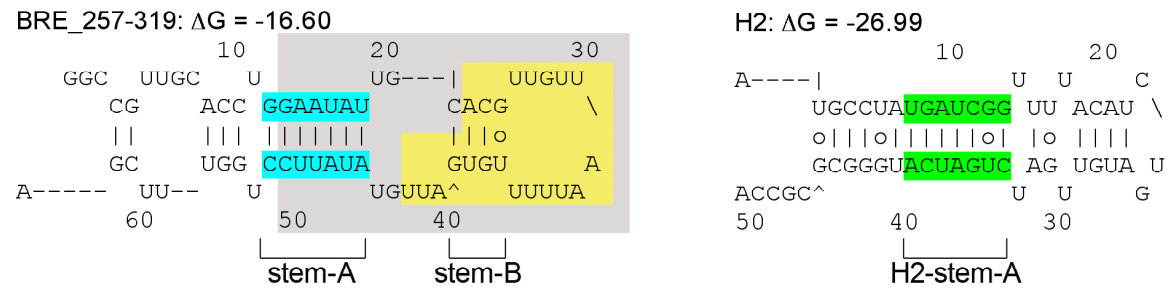

Fig. 3.16: Predicted secondary structures of BRE_257-319 and H2. In the predicted secondary structure of BRE_257-319 (left), the area in grey indicates the sequence that is conserved among different Drosophila species; the area in yellow shows the miR-308 binding target. The predicted secondary structure of $\mathrm{H} 2$ (right) contains a putative stem structure (marked in green, H2-stem-A), which shows structural similarity to the stem structure in BRE_257-319 (marked in blue, stem-A). Secondary structure predictions were performed using the mfold program (version 2.3).

\subsubsection{Mutations in the miR-308 target site abolish BCD-mediated translational repression}

To investigate the role of miR-308 in cad mRNA translational regulation, I introduced 4 point mutations in the Dm3' that lead to the disruption of miR-308 binding. Two base pairs in stem-B, nt 281C, 282G and nt 294G, 295U, were changed into 281G,282U and 294A,295C, so that the overall structure may be retained (Fig. 3.17, A). The resulting sensor, 38F-Dm3'mut, should be incapable of interacting with miR-308 through that site. I analysed the expression levels of the sensor 38F-Dm3'mut in embryos and in comparison to sensor 38F-Dm3' (Fig. 3.17, B). Embryos deriving from females with the sensor 38F-Dm3'mut show about $50 \%$ increase in dnGFP fluorescence intensities as compared to sensor 38F-Dm3', which carries the wt cad 3'UTR (Fig. 3.17, C, $100 \% \pm 3.6$ in embryos deriving from 38F-Dm3'mut/+;nos-GAL4:VP:16 females; $53.1 \% \pm 2.2$ in embryos deriving from 38F-Dm3'/+;nos-GAL4:VP:16 females; $\left.p=3.10 \times 10^{-13}\right)$. These results indicate a de-repression, which is most likely due to loss of miR-308 binding.

38F-Dm3'mut sensor transcripts lost their susceptibility to BCD, because dnGFP fluorescence of 38F-Dm3'mut is unaffected by BCD isoform G (Fig. 3.17, C; maternal genotype: UAS-BCDG ${ }^{\mathrm{FM} 6} /+; 38 \mathrm{~F}-\mathrm{Dm} 3$ 'mut/+;nos-GAL4VP:16/+; $p=0.46$ ). Next । asked, whether the other homeodomain-bearing BCD isoforms are capable of mediating the repression of 38F-Dm3'mut, perhaps through binding to other sites in the mutated cad 3'UTR. The analysis, including BCD isoform D, E or F, revealed that none of these isoforms are able to downregulate dnGFP in the 38F-Dm3'mut sensor (Fig. $3.17, \mathrm{D})$. It seems therefore that the nucleotides that have been mutated in 38FDm3'mut are crucial for translational repression by any homeodomain-bearing isoform of BCD. 
A

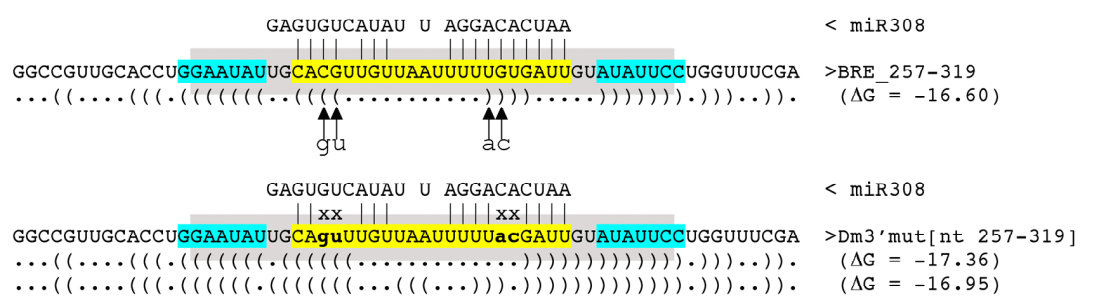

B

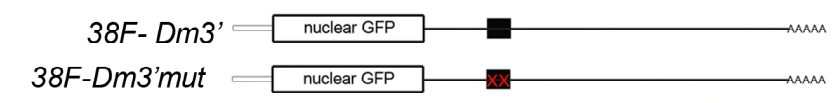

C
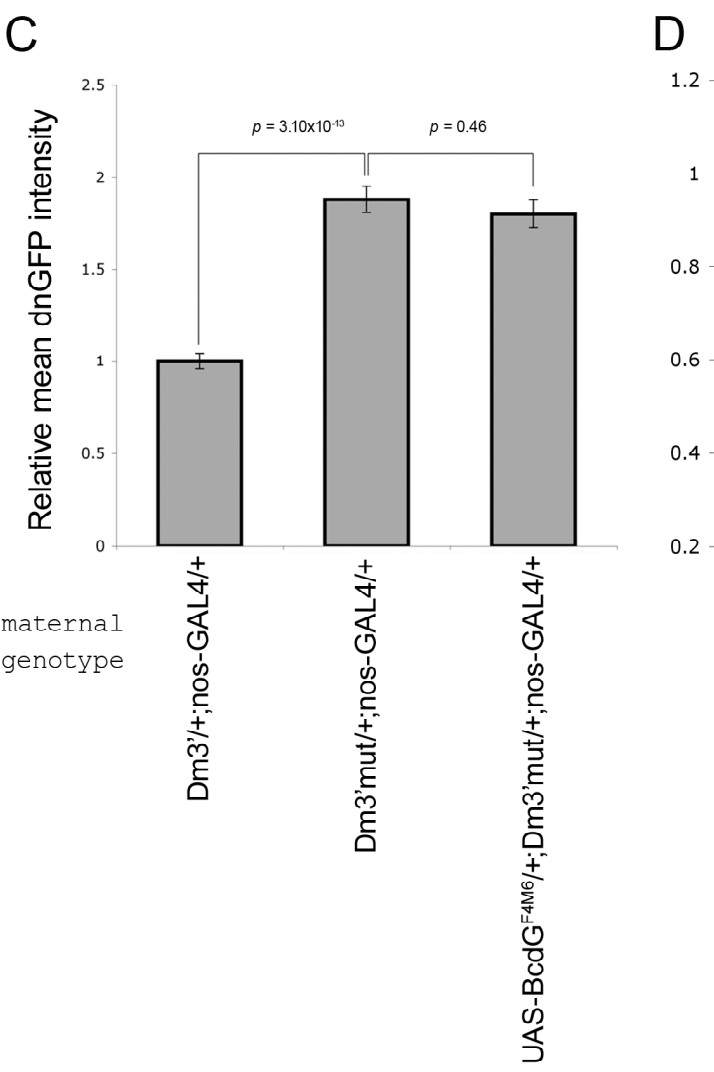

$\square$ sensor only
$\square+$ BCD isoform
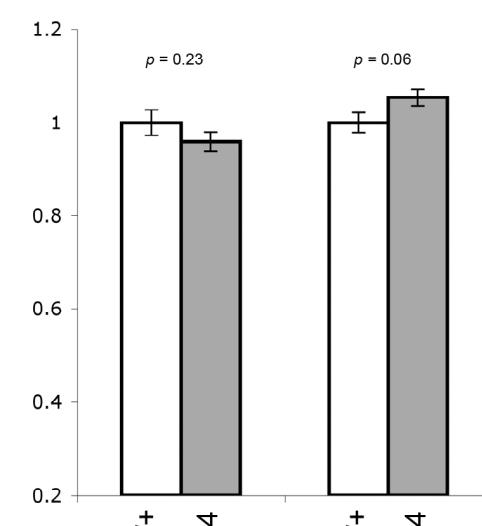

$p=0.04$

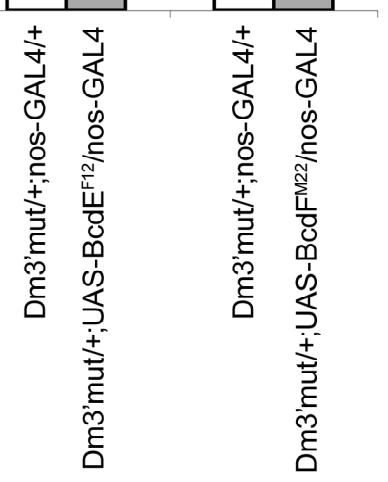

Fig. 3.17: Translational reporter assay for repression of the mutated cad 3'UTR in sensor 38F-Dm3'mut by different BCD isoforms. (A) miR-308 binding to wt target in BRE 257-319 of Dm3' (indicated in yellow) and mutated target Dm3'mut (mutated nt in small, bold letters). The predicted secondary structures (Vienna format) are indicated below the sequence of the wt and mutated BRE_257-319. The area in grey indicates the sequence, which is conserved among different Drosophila species. Blue regions form the putative stem structure stem-A. Nucleotides that were changed in 38F-Dm3'mut are indicated by arrows. (B) Schematic overview of the transgenic reporter transcripts. (C) Average dnGFP intensities of 38F-Dm3'mut are roughly $50 \%$ higher as compared to 38F-Dm3' $\left(p=3.10 \times 10^{-10}\right)$ and unaffected by the presence of UAS$\mathrm{BCDG}^{\mathrm{F} 4 \mathrm{M} 6}(p=0.46)$. (D) Average dnGFP intensities of 38F-Dm3'mut in the presence of BCD isoform $\mathrm{D}(p=0.23), \mathrm{E}(p=0.06)$ and $\mathrm{F}(p=0.04)$. The $p$ value obtained for isoform $\mathrm{F}$ is marginally above the $5 \%$ threshold of statistical significance. This difference could not be reproduced in independent measurements and the dnGFP levels of the 38F-Dm3'mut sensor in the presence of isoform $\mathrm{F}$ are therefore regarded as not different. Bars represent relative mean dnGFP values \pm SE, statistical significance was analysed using Students t-test. dnGFP intensity values were measured from region of interests of identical size from the surface of blastoderm embryos at nuclear division cycle 11. 


\subsubsection{Translational repression of 38F-BRE_257-319 and 38F-H2 by BCD isoform F}

Mutations that disrupt the miR-308 target site in 38F-Dm3'mut not only abolish miRNAinduced repression, but also result in loss of BCD-dependent repression by all isoforms. This could suggest that miR-308 activity may be necessary for BCD to function as a translational repressor. However, the mutations in sensor 38F-Dm3'mut may have changed the structure of the BRE_257-319 fragment or affected BCD binding directly (further discussed in 4.1.3). The $\mathrm{H} 2$ fragment contains a BCD-binding element (see 3.2.2.2), which is most likely independent of miRNA function, because $\mathrm{H} 2$ lacks predicted miR-308 binding target sites. Using this fragment I wanted to investigate whether there might be a difference in the translational output by a BCD isoform with and without d4EHP-binding domain (BCD isoform $G$ and $F$, respectively) in the absence of miRNA activity.

Sensor 38F-BRE_257-319 or sensor 38F-H2 were co-expressed with isoform $F$ (yw;38F-H2/+;UAS-BCDF ${ }^{\mathrm{M} 22} /$ nos-GAL4:VP16) or with isoform G (UASBCDG $^{\mathrm{F} 4 \mathrm{M} 6} /+; 38 \mathrm{~F}-\mathrm{BRE} \_257-319 /+;$ nos-GAL4:VP16/+) and the dnGFP levels analysed in embryos deriving from those females. Fluorescence levels of sensor 38F-BRE_257319 show a reduction of about $20 \%$ induced by $B C D$ isoforms $G(80.6 \% \pm 1.4$ of initial dnGFP levels, $\left.p=4.8 \times 10^{-15}\right)$ and isoform $F(75.3 \% \pm 1.2$ of initial dnGFP levels, $p=2.8 \times 10^{-20}$ ) (Fig. 3.18, A and B). In contrast, fluorescence levels of sensor 38F-H2 were reduced to only $87 \% \pm 2.9$ of the initial sensor levels by $B C D$ isoform $G$ $(p=0.001)$ and $93.9 \% \pm 1.8$ by BCD isoform $\mathrm{F}(p=0.03)$ (Fig. 3.18, D and $\mathrm{E})$. These results show that $B C D$ isoforms $G$ and $F$ are both able to repress dnGFP levels of sensor 38F-BRE_257-319, but repression of sensor 38F-H2 is significantly lower, with repression by $\mathrm{BCD}$ isoform $\mathrm{F}$ being marginally significant.

In comparison to the BRE_257-319 fragment, interaction of BCD with $\mathrm{H} 2$ is less efficient in mediating the translational repression. This is consistent with the weaker binding affinity of the BCD homeodomain to the $\mathrm{H} 2$ fragment seen in EMSAs. Furthermore, $B C D$ isoform $F$ seems to be less effective than isoform $G$ but is still capable of repressing sensor 38F-H2. This result suggest the existence of mechanism for BCD-mediated translational repression, which is independent of miR-308 and d4EHP. 
A
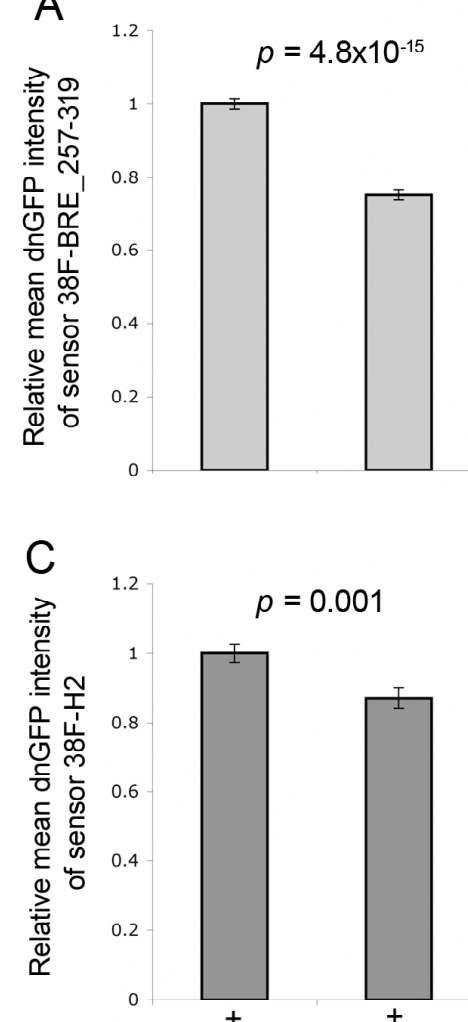

maternal genotype
B

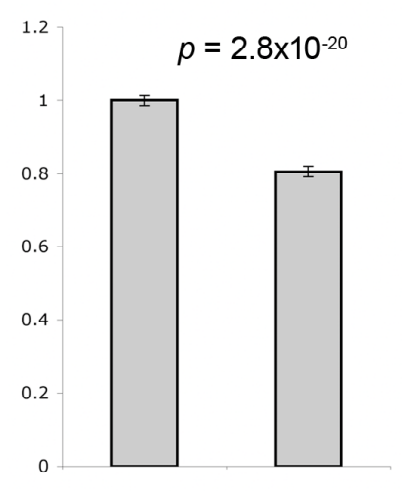

D

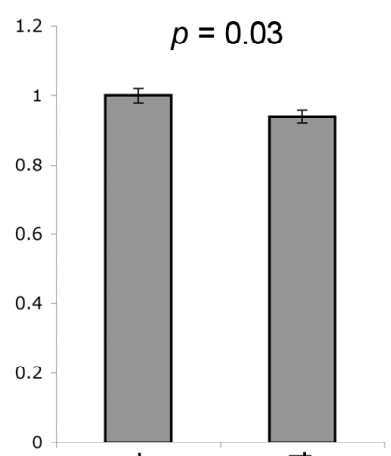

Fig. 3.18: Translational reporter assay for repression of BRE_257-319 and H2 by BCD isoforms $\mathbf{G}$ and $\mathbf{F}$. (A) Mean dnGFP intensities of sensor 38F-BRE_257-319 in the presence of $\mathrm{BCD}$ isoform $\mathrm{G}\left(p=4.8 \times 10^{-15}\right)$ and $\mathrm{BCD}$ isoform $\mathrm{F}\left(\mathrm{B}, p=2.8 \times 10^{-20}\right)$ are reduced by about $20 \%$ compared to dnGFP levels of the sensor alone. Mean dnGFP intensities of sensor 38F-H2 are reduced by about $10 \%$ in the presence of BCD isoform $G(C, p=0.001)$ and $B C D$ isoforms $F(D, p=0.03)$ compared to the dnGFP levels of the sensor alone.

\subsubsection{Conclusions}

The cad 3'UTR contains a predicted target site for miR-308 (Brennecke et al., 2005; Stark et al., 2005). miR-308 has previously been cloned from embryonic total RNA extracts, showing low expression levels, which were undetectable by Northern blotting 
(Aravin et al., 2003; Leaman et al., 2005). Here I show that a mutation in the miR-308 binding target of the cad 3'UTR elevates the expression levels of a sensor as compared to the wt 3'UTR. These observations suggest that miR-308 represses expression of a sensor that carries the cad 3'UTR. Because the miR-308 target site is located in the BCD binding fragment BRE_257-319, miR-308 may be involved in BCDmediated control of cad translational repression. To address this question, I analysed whether the 38F-Dm3'mut sensor, which carries four point mutations in the miR-308 binding site, is susceptible to BCD repression. None of the BCD isoforms showed an effect on the dnGFP levels of the 38F-Dm3'mut sensor.

This finding would be consistent with a mechanism for BCD-mediated translational repression that also involves miR-308. However, mutations present in Dm3'mut may have disrupted the RNA structure due to the initial mutagenesis design. Secondary structure predictions of the mutated cad 3'UTR fragment revealed that the introduced sequence modifications could also influence the secondary structure of stem-A (Fig. A4.3). Hence, it cannot be excluded that along with the destruction of the miR-308 target site, structural changes in the RNA occurred that altered the putative BCDbinding motif. Therefore, the mutations in 38F-Dm3'mut might change the secondary structure, which might be essential for BCD action, or the mutations may directly affect BCD binding.

The translational repression of sensor 38F-Dm3' by BCD isoforms that lack the d4EHPbinding domain ( $B C D$ isoforms $E$ and $F$ ) could indicate the existence of a d4EHPindependent mechanism. I wanted to ask, whether the effects of miRNA action are important for translational repression by such BCD isoforms. Sensor 38F-H2, which is free of putative miRNA target sites in Drosophila, is translationally repressed by BCD isoform $\mathrm{F}$. The level of repression, however, is very low compared to repression by $\mathrm{BCD}$ isoform $\mathrm{G}$ and only marginally significant. This shows that the repression of $38 \mathrm{~F}-$ $\mathrm{H} 2$ mediated by $\mathrm{BCD}$ isoform $\mathrm{F}$ is less effective than the repression mediated by isoform $\mathrm{G}$. Therefore, the absence of d4EHP function reduces $B C D$ activity with sensor transcripts that lack miRNA target sites. It is possible that the presence of miRNA activity may aid BCD activity, however is dispensable for BCD-mediated translational repression. 


\section{General Discussion}

\subsection{Insights into translational control of cad in Drosophila}

Rivera-Pomar et al. (1996) reported binding of the BCD homeodomain to a fragment corresponding to nt 66-185 (here termed BRE_66-185) of the cad 3'UTR. The authors presented in vivo data from a CAT reporter assay with cad 3'UTR fragments showing reduction of translational efficiency in the presence of BCD. Only BCD derivates containing the homeodomain could interact with the full cad 3'UTR (Rivera-Pomar et al., 1996). Sequence-specificity of BCD binding to the BRE_66-185 fragment using competition experiments were not performed. Subsequently, this RNA fragment was used to analyse different BCD protein regions that are involved in cad translational repression and the DNA/RNA-binding properties of the BCD homeodomain (Niessing et al., 1999; Niessing et al., 2000).

Dubnau and Struhl (1996) showed that a 342-nucleotide fragment (here termed BRE_210-553) of the cad 3'UTR is necessary and sufficient for BCD-dependent translational repression of a reporter transcript. They provided evidence for at least two binding sites in the BRE_210-553 fragment shown by direct binding of the BCD homeodomain to nt 210-318 and to nt 319-433 of the cad 3'UTR in cross-linking experiments. In the same assay, binding to a fragment containing cad 3'UTR nt 434533 did not occur. Reporter constructs containing nt 1-318 and 553-940, or nt 1-443 did not mediate anterior repression of the reporter (see Dubnau and Struhl, 1996, Fig. 2c). Hence, the authors suggested that the full region of the BRE_210-553 element is necessary for translational repression in vivo.

The binding specificity of the BCD homeodomain to a fragment within BRE_210-553, spanning only the $43 \mathrm{nt}$ (here termed BRE_210-253), was further characterized in vitro using electrophoretic mobility shift assays. The authors reported sequence-specific binding of the BCD homeodomain to this fragment (Chan and Struhl, 1997).

In my translational reporter assay, sensors carrying the BRE_257-319 fragment can interact with $B C D$ and mediate translational repression of the reporter, while four point mutations, in this fragment within region nt 281-295 (sensor 38F-Dm3'mut) result in loss of BCD-mediated repression. Therefore, the region 281-295 of the cad 3'UTR 
contributes to BCD-binding in the BRE_257-319 fragment, which is sufficient to mediate translational repression of reporter transcripts by BCD.

This finding is in conflict with the results obtained with the reporter assay of Dubnau and Struhl (1996). Reporter constructs carrying nt 1-318 and 553-940 and nt 1-443 of the cad 3'UTR (including BRE_257-319) did not mediate anterior repression of the reporter in the embryo. One possible explanation is that their reporter assay was not sensitive enough to detect subtle differences in translational repression levels mediated by different BCD-binding elements and required the presence of multiple BCD-binding sites to show anterior repression by BCD. This would imply the presence of several BCD-binding sites, with additive effects on translational repression of this reporter. Alternatively, proper folding of the BCD-binding element might have been disrupted in these reporter derivates. The reporter was capable to detect translational repression only when the complete BRE_210-553 fragment was present.

The use of a fluorescent protein in my reporter assay, combined with the ectopic expression of BCD, allows the quantitative analysis of small but significant differences in expression. Thus it was possible to detect loss of BCD repression in a fragment with only four nucleotide changes. It is possible that previously undetected BCD-binding sites in the cad 3'UTR, which function in vivo but are of lower efficiency in mediating repression, may be identified by using my translational reporter assay.

Most cis-regulatory elements that regulate the translational output of an RNA lie within untranslated regions of mRNAs. These regions are usually under lower selective pressure to maintain their primary sequence than the coding sequences. One of the reasons might be that cis-regulatory elements can be encoded by structural features of the RNA molecule, which can be formed by different primary sequence (reviewed in Schroeder et al., 2004). The BCD-binding signal might be encoded by secondary structures of RNA fragments. The BRE_257-319 and H2 fragments do not share sequence similarities, however, these fragments share a similar stem in their predicted secondary structures, stem-A in BRE_257-319 and H2-stem-A in H2. Both fragments are bound in vitro by the BCD homeodomain and mediate BCD-dependent repression of sensor transcripts in embryos.

Could this stem structure "mimic" the structure of the BCD double stranded (ds) DNA target and therefore co-opt the homeodomain's DNA-binding properties for RNA binding? DNA recognition of the BCD homeodomain occurs through direct contacts in the major groove of the DNA targets, which are mediated by the residues K50 and R54 
(Baird-Titus et al., 2006; Niessing et al., 2000). Interestingly the side-chains of K50 and R54 seem to show some flexibility in their precise positioning during the contact making of the DNA target (Baird-Titus et al., 2006). dsRNA normally adopts A-form helices, displaying a major groove which is narrower and deeper than the B-form helices of dsDNA and cannot be accessed by an $\alpha$-helix as occurs in dsDNA binding. However, a sequence of purines can cause dsRNA to adopt a different helical conformation (socalled A' conformation), with an expanded major groove, similar to that of B-form dsDNA (Bullock et al., 2010).

The fact that the BCD homeodomain binds to RNA fragments like BRE_257-319 and the $\mathrm{H} 2$ fragment, but not to unstructured RNA fragments (CU58mer) could be an indication for the preference of the homeodomain to bind to RNA molecules that contain helical structures. It is conceivable that within the physical constraints of the BCD homeodomain the binding occurs to an RNA molecule that is mimicking the DNA target and allowing the homeodomain to access the major groove of the RNA helix, e.g. A'-form helix. Perhaps binding to the RNA depends primarily on a specific structure rather than a specific sequence, which would explain how the BCD homeodomain binds to different RNA sequences. At the same time, it is possible that the BCD homeodomain requires specific base contacts with a few bases of the RNA, especially if the binding to RNA occur in a similar way as the DNA binding.

Whether the stem structures in the BRE_257-319 or H2 fragments adopt an A'-form or B-form-like conformations can only be revealed by crystallizing the RNA:HD complex and inferring the structure of the complex. As a first approach, it would be interesting to further investigate the role of the stem structure of the BRE_257-319 or the H2 fragment in BCD RNA binding by testing point mutations that interfere with binding by using the in vivo translational reporter assay.

A more indirect indication can be drawn from the analysis of the Tc'cad 3'UTR, which does not interact with $B C D$ in the translational reporter assay and cannot interact in vitro with the BCD homeodomain. I could not find any structural similarities in the Tc'cad 3'UTR to the BRE_257-319 fragment using the RNAforester program (data not shown). Thus, the remaining sequences in the Tc'cad mRNA have to be further analysed for putative BCD binding element(s). In fact, a region situated in the ORF spanning nt 451-485 of the Tc'cad cDNA (numbering begins from the first $\mathrm{nt}$ in the cDNA) shows structural similarities to the BRE_257-319, but can at this point only be suggested as a promising candidate that observed BCD-dependent translational repression. 
Another discrepancy between my results and previously published data concerns the binding specificity of the BCD homeodomain to the cad 3'UTR. Chan and Struhl (1997) reported specific binding of the BCD homeodomain to an RNA fragment containing $\mathrm{nt}$ 210-253 of the cad 3'UTR (here termed BRE_210-253). The BRE_210-253:BCD homeodomain-complex was undisturbed in the presence of a 100-fold excess of Tuba -1 RNA. From Figure 1a of Chan and Struhl (1997), we deduce that this fragment was approximately 300 nucleotides long, similar in size to the fragment of Tub $\alpha-1$ RNA. In my competition experiments, binding of the BCD homeodomain to a similar sized fragment, the BRE_164-339 (a 214 nt fragment, which includes nt 210253), occurred with dramatically lower specificity. The interaction of the BCD homeodomain with the BRE_257-319 fragment was also weak and showing relatively low specificity in competition experiments. This interaction was confirmed using my in vivo translational reporter assay, which strongly supports a functional interaction of $B C D$ with this fragment. In vitro the binding of the BRE_257-319 fragment to the BCD homeodomain seems to be easily competed by unrelated RNAs such as shSV40. However, BCD is unable to bind to SV40 3'UTR with high affinity in the EMSA experiments and the in vivo results show that expression of the sensor carrying the SV40 3'UTR is unaffected by BCD. This might also suggest that in vivo RNA-binding specificity of the full-length BCD protein is higher than homeodomain binding in vitro.

My results suggest that recruitment of additional factor(s) may increase the RNAbinding specificity of the protein in vivo. This means that perhaps the presence of other sites of the BCD protein or factors acting in trans help in conferring this specificity. 


\subsection{Alternative mechanisms of translational control of cad mRNA}

\subsubsection{Evidence for d4EHP-independent cad translational repression}

The role of d4EHP in cad translational repression by BCD has been previously identified through genetic and biochemical analysis (Cho et al., 2005). The authors showed that $B C D$ derivatives carrying mutations in the d4EHP-binding domain were unable to exert translational regulation on cad mRNA. These mutants included $b c d^{\text {Y } 66 \mathrm{~A}}$ and $b c d^{L 73 R}$ that carried changes in the amino acid residues $Y 66$ and L73. In contrast, in my experiments, BCD isoforms $E$ and $F$, which lack the 4EHP-interaction domain, were capable to mediate translational repression of sensors like 38F-Dm3' (Tab. 4.1).

Perhaps, $B C D$ isoforms $E$ and $F$ are able to exert different protein functions and are thus to mediate the translational repression of sensor 38F-Dm3. This could be due to different properties imparted by the $\mathrm{N}$-terminal domain, which is present in BCD isoforms $\mathrm{G}$ and $\mathrm{D}$, but absent in $\mathrm{E}$ and $\mathrm{F}$. Alternatively, translational repression of cad with $b c d^{\mathrm{Y} 66 \mathrm{~A}}$ and $b c d^{\mathrm{L} 73 \mathrm{R}}$ without d4EHP was not detectable due to visualisation with $\alpha$ CAD antibodies. My translational reporter assay is a more sensitive assay for translational repression. $B C D$ isoforms $E$ and $F$ are ectopically expressed during oogenesis and might therefore be able to show an effect on translation of the sensor.

Niessing et al. (1999) used a dicistronic reporter assay to investigate the role of BCD protein domains other than the homeodomain in cad translational repression. The first cistron encoded the chloramphenicol acetyltransferase (CAT), the second cistron encoded luciferase (Luc). Both cistrons were separated by an internal ribosome entry site (IRES), making it possible to distinguish between cap-dependent (CAT activity) and cap-independent (Luc-activity) translational repression. The 3'UTR of this reporter contained the BBR of the cad 3'UTR, which was previously characterized by RiveraPomar et al. (1996). This reporter was then co-transfected with effector plasmids containing different truncated BCD proteins. The deletion analysis with the truncated BCD proteins showed that $\mathrm{C}$-terminal deletions up to the PEST domain and $\mathrm{N}$-terminal deletions up to close to the homeodomain are able to exert translational repression of the reporter. Of these N-terminal deletions, $\mathrm{Bcd}^{77-202}$ (deletion of aa 1-76 and 202-489; the full length $B C D$ protein must refer to $B C D$ isoform $D$, which contains 489 aa) gave a weaker repression of translation of the reporter than $\mathrm{Bcd}^{41-202}$. The protein domains that 
lie between amino acid 41 and 77 are the SID (aa 52-61) and the d4EHP-binding domain (65-77), therefore the truncated protein $\mathrm{BCD}^{77-202}$ lacks these domains.

These findings agree with my results, suggesting that BCD can mediate translational repression of the reporter in the absence of the d4EHP-binding domain: this is seen both with truncated $\mathrm{Bcd}^{77-202}$ with the CAT/Luc reporter in S2 cells and by BCD isoforms $\mathrm{E}$ and $\mathrm{F}$ in my translational reporter assay in embryos. In both approaches translational repression of sensors with the cad 3'UTR was assayed out of the natural context of early embryogenesis. It is therefore possible that additional mechanisms available in S2 cells and during oogenesis were employed to mediate repression.

\subsubsection{The putative role of miR-308 in BCD-dependent cad translational repression}

The de-repression of sensor 38F-Dm3'mut, which contains four point mutations in the miR-308 binding target site, provides the first evidence for a role of miR-308 in control of cad mRNA expression in Drosophila. Interestingly, the miR-308 target site is located within a characterized BCD-binding fragment. It seems though that miRNA activity may be a redundant mechanism for BCD translational repression, as demonstrated with sensor 38F-H2, which lacks predicted miRNA binding targets, but mediates low repression (discussed further below). For the putative involvement of miR-308 in translational repression, these findings can be interpreted in three ways:

(1) BCD and miR-308 act independently from each other. While miR-308 reduces overall mRNA levels, BCD mediates translational repression to prevent CAD protein from being expressed at the anterior part of the embryo. Both share an overlapping interaction site in the cad 3'UTR. The mutations introduced in 38F-Dm3'mut therefore destroyed both the BCD-mediated and the miR-308-mediated translational regulation.

(2) miR-308 binding is involved in BCD-mediated translational repression, but dispensable. BCD can follow other mechanisms to mediate translational repression, however the absence of miR-308 activity results in a reduced BCD-dependent repression as it was observed with senor 38F-H2. The loss of BCD-dependent repression of sensor 38F-Dm3'mut could be explained by changes in the RNA secondary structure that affect BCD binding. 
(3) miR-308 binding is necessary for the BCD-mediated translational repression. In this case, it should be possible to regain BCD-dependent translational repression of the 38F-Dm3'mut sensor by providing a miRNA that carries nucleotide changes that are complementary to those in Dm3'mut mutations.

In a variation of interpretation (3), I speculated that translational repression by BCD isoform $F$ may entirely depend on the miRNA interaction, while BCD isoform $G$ can mediate the repression through the d4EHP-mediated mechanism. To test this hypothesis, I analysed sensor 38F-H2 in the presence of $B C D$ isoform $F$. BCD isoform $\mathrm{F}$ shows a less translational repression of sensor $38 \mathrm{~F}-\mathrm{H} 2$ in comparison to $38 \mathrm{~F}$ BRE_257-319. However, the translational repression of $38 \mathrm{~F}-\mathrm{H} 2$ by $\mathrm{BCD}$ isoform $\mathrm{F}$ is at the border of statistical significance. At this point these results are not conclusive and a more detailed investigation of fragment $\mathrm{H} 2$ with $\mathrm{BCD}$ isoform $\mathrm{F}$ will be required. If it will be shown that $B C D$ isoform $F$ is indeed incapable to mediate translational repression of sensor $38 \mathrm{~F}-\mathrm{H} 2$, then this would be the first indication for a miRNA dependence of translational repression by a 4EHP-independent mechansim.

Tab. 4.1: Presence of d4EHP-binding domain in different BCD isoforms and their ability to mediate translational repression of different sensors. Only isoform $G$ and $D$ contain a d4EHP-binding domain (indicated by + ). ${ }^{*}$ The translational repression of sensor $38 \mathrm{~F}-\mathrm{H} 2$ remains to be confirmed as current measurements were found to be only marginally significant. $(+)$ indicates repression; (-) indicates no repression; n.a. = not analysed.

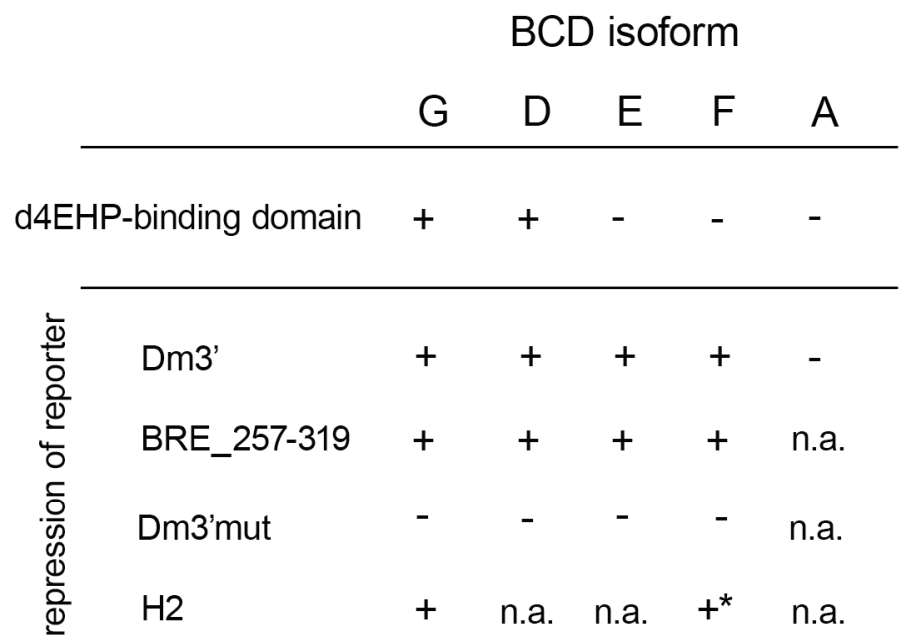




\subsection{Implications for the evolution of cad translational regulation}

Drosophila cad promotes posterior identity and it is crucial to prevent its expression in the anterior of the early embryo (Macdonald et al., 1986; Rivera-Pomar et al., 1995; Schulz and Tautz, 1995). The anterior determinant BCD has acquired the function to repress cad mRNA translationally (Rivera-Pomar et al., 1996; Dubnau and Struhl, 1996). BCD is an evolutionary novelty of higher dipteran flies, thus, different factors or mechanisms must mediate this process in other insect species. For example the parasitic wasp Nasonia, which is thought to have evolved long-germ embryogenesis independently from flies, localizes cad mRNA at the posterior pole of the embryo, which then creates a posterior to anterior concentration gradient of the cad mRNA and most likely also of the CAD protein (Olesnicky et al., 2006) (Fig. 4.1).

In the beetle Tribolium, Tc'cad is maternally expressed and distributed uniformly in the early embryo. Unlike in Drosophila, the Tc'CAD protein is detected uniformly at early blastoderm stages and shortly after the protein becomes restricted to the posterior part, resembling a posterior to anterior gradient (Schulz et al., 1998). The onset of zygotic Tc'cad expression is not clearly distinguishable from the maternal expression at the time of the protein gradient formation. The earliest zygotic Tc'cad transcripts, however, occur at a rather early blastoderm stage (person. comm., A. Peel). The repression of anterior Tc'cad expression is thought to be mediated by two zygotic genes, Tc'zen-2 and Tc'mex-3, and is suggested to represent the ancestral state of cad translational repression in insects (Schoppmeier et al., 2009) (see below and Fig.4.1).

In order to gain further insight into when and how cad translational control evolved from a still undefined ancestral state to the BCD-dependent mechanism observed in Drosophila we can gather clues from comparisons with other related insect species. Presumably there are two aspects in the process of cad translational repression that have evolved or may be conserved among different species: (1) the RNA target sequence in the cad 3'UTR and (2) the mode of cad regulation, e.g. the ability of an anterior factor to bind to this sequence and mediate translational repression.

To address the former, I analysed the regulation of cad 3'UTRs of the dipteran Haematopota and the beetle Tribolium in the presence of BCD using the translational reporter assay. The Haematotpota cad 3'UTR is translationally regulated by BCD in Drosophila and the BCD homeodomain directly binds to the $\mathrm{H} 2$ fragment of the Hp'cad 3'UTR in vitro. In contrast, sensors with the Tribolium cad 3'UTR are not regulated by $B C D$, therefore the previously reported BCD-dependent translational repression of 
Tc'cad in transgenic Drosophila embryos (Wolff et al., 1998) most likely depends on unidentified sequences in the 5' UTR or the coding sequence of the Tc'cad mRNA.

The discovery that the Hp'cad 3'UTR can mediate BCD-dependent translational repression in Drosophila suggests the existence of an RNA signal that is conserved among these insect species. Although there are no extensive sequence similarities, there are hints, provided by comparisons of the 3'UTR fragments BRE_257-319 and $\mathrm{H} 2$, that the secondary structure may contribute to this conserved BCD-recognition signal. This hypothesis may be tested through the identification of putative conserved secondary structures in the cad 3'UTRs of other related insect species and the investigation of the ability of these cad 3'UTR fragments to confer translational repression by $B C D$.

The second aspect in the process of cad translational repression concerns the ability of an anterior factor to bind to the 3'UTR. Conserved 3'UTR elements indicate the existence of similar or related factors, repressing the mRNA bearing these elements. Which factor(s) might be involved in the translational repression of the cad homologues in different insect species and do they recognize the same RNA-elements? BCD arose through a gene duplication of a Hox3/zen-like gene progenitor in the lineage of the cyclorrhaphan flies (Falciani et al., 1996; Stauber et al., 1999). The 4EHP-binding domain of BCD is highly conserved among different Drosophilid species, and therefore cad translational repression most likely employs the 4EHP-mediated mechanism of translational repression in all Drosophilids. The sister gene of $b c d$, zen seems to be a good candidate for cad translational repression in other insect species. In dipterans like Empis livida, Haematopota pluvialis or Clogmia albipunctata, the Hox3/zen-like genes share more similarities with zen genes and lack a 4EHP-binding domain (Stauber et al., 2002). The homeodomain of Haematopota zen (Hp'zen) does not contain the amino acid residue (R54), which has been shown to define the RNA-binding specificity in BCD (Niessing et al., 2000; Stauber et al., 2002). Hp'cad and Hp'zen mRNAs are maternally expressed, however it is not clear, how the mRNAs are distributed in the embryo and whether Hp'ZEN may regulate Hp'cad translation.

One of the zen orthologues in Tribolium, Tc'zen-2, has been shown to repress Tc'cad mRNA, but so far there is no evidence for a direct interaction of Tc'ZEN-2 with the Tc'cad mRNA. Tc'ZEN-2 does not contain a 4EHP-binding domain and Tribolium 4EHP RNAi does not affect Tc'cad mRNA translation (Schoppmeier et al., 2009). Tc'ZEN-2 also lacks Arginine at position 54 of the homeodomain. It is therefore unclear if the Tribolium zen orthologue is able to mediate cad translational repression directly. 
Taken together it seems unlikely that zen proteins in other insects would confer translational repression in a similar way as BCD does in Drosophila, although a more detailed analysis of $T c^{\prime}$ cad and Hp'cad translational repression should be undertaken to address this question. Because zen proteins do not seem to fulfil the requirements that have be shown to be necessary for BCD RNA-binding, it is likely that ancestrally one or more unknown factors, perhaps in concert with ZEN, were employed for cad translational repression. The analysis of the BCD isoforms has revealed that a d4EHPindependent mechanism(s) exist for cad translational repression in Drosophila. I suggest that this mechanism might represent aspects of an ancestral state of cad translational repression. It will therefore be important to identify the factors involved, as they might have a role during translational repression of cad homologues in other insect species.

Which evolutionary event preceded the other: the amino acid changes in the BCD homeodomain that lead to the BCD RNA-binding properties or RNA nucleotide adaptations in the cad transcript, creating a binding signal for a DNA-binding protein? The adaptability of RNA molecules is thought to be very high; it is even possible to create RNA sequences that artificially adopt DNA-binding target signals of transcription factors that were not known to bind to RNA (Cassiday and Maher III, 2002). Conserved functional interactions of the Drosophila and Haematopota 3'UTR fragments with BCD suggest that there was selective pressure on these 3'UTR sequences to maintain a signal for translational repression. While, sequence similarities are largely lost, the predicted secondary structures of BRE_257-319 and H2 show overall similarities. However, it is not clear to what extent the secondary structures and/or perhaps specific nucleotides within these structures may contribute to $B C D$ homeodomain binding. $A$ detailed analysis of these two structures may help to reveal which nucleotide substitutions within the secondary structure can be tolerated and which reduce or abolish BCD RNA-binding. Such an analysis might give clues about the adaptations of RNA sequences and the switch to an anterior regulator that used to be a DNA-binding protein, like the Hox3/zen-like derived bcd gene, which was co-opted into a new function in translational regulation. 


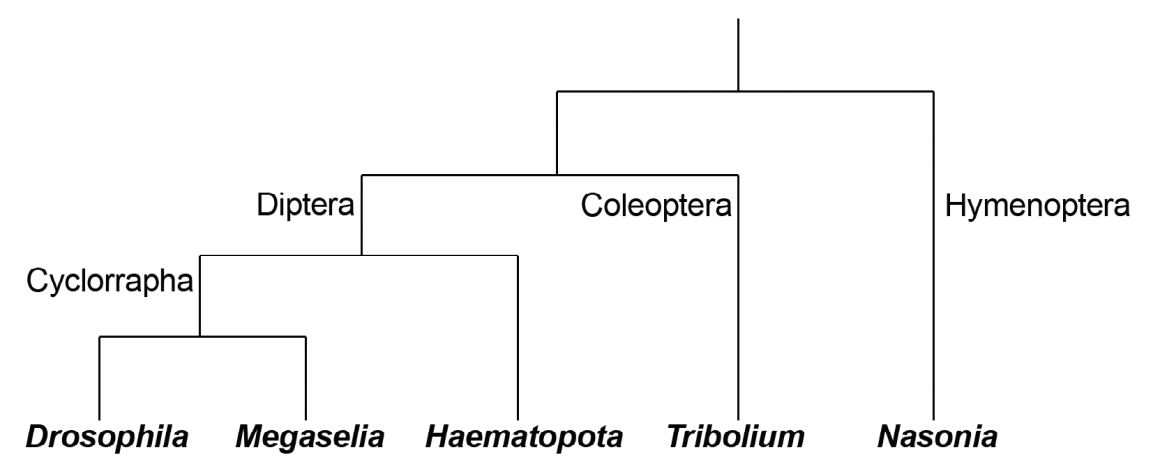

\begin{tabular}{|c|c|c|c|c|c|}
\hline $\begin{array}{l}\text { Hox3/zen-like } \\
\text { genes }\end{array}$ & $\begin{array}{l}b c d^{\text {mat }} \\
z e n^{z y g} \\
z 2^{<y y}\end{array}$ & $\begin{array}{l}\text { Mab'bcd'mat } \\
\text { Mab'zen }\end{array}$ & Hp'zen'mat, (zyg?) & $\begin{array}{l}\text { Tc'zen-1zyg } \\
\text { Tc'zen-2'yyg }\end{array}$ & $?$ \\
\hline 4EHP gene & + & $?$ & $?$ & + & $?$ \\
\hline $\begin{array}{l}\text { Mode of cad } \\
\text { mRNA } \\
\text { regulation }\end{array}$ & $\begin{array}{c}\text { cad }^{\text {mat }} \\
\text { transl. repr. } \\
\text { mediated by } \\
\text { 4EHP and } \\
\text { BCD }\end{array}$ & $\begin{array}{c}\text { no mat. cad; } \\
\text { expression } \\
\text { regulated } \\
\text { zygotically }\end{array}$ & $\begin{array}{c}? \\
\left(c a d^{\text {mat }}\right. \\
\text { present } \\
\text { in oocytes) }\end{array}$ & $\begin{array}{c}\text { cad }^{\text {mat }} \\
\text { repr. by } \\
\text { Tc'MEX-3 } \\
\text { and } \\
\text { Tc'ZEN-2 }\end{array}$ & $\begin{array}{c}\text { cadmat } \\
\text { mRNA } \\
\text { posteriorly } \\
\text { localized }\end{array}$ \\
\hline
\end{tabular}

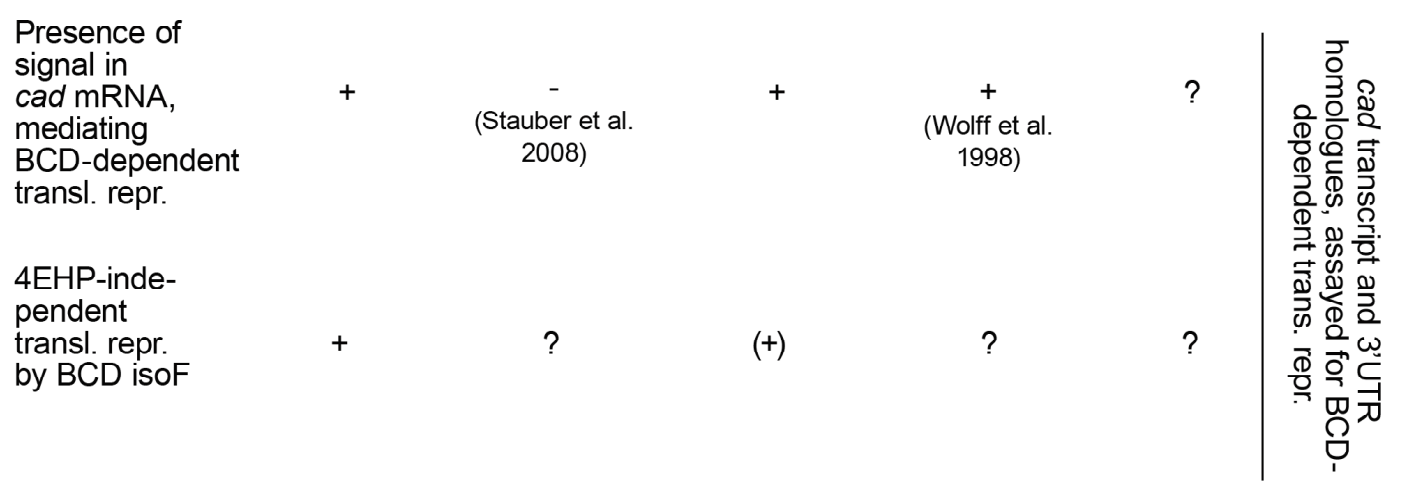

Fig. 4.1: Phylogeny of insect species (after Savard et al., 2006) discussed with features of cad translational repression mapped on. Uncertain characters states have been left clear (?) or are marked if further experiment are required to confirm the result $\left(^{*}\right)$. mat $=$ maternal; zyg $=$ zygotic; trans. repr. = translational repression. 


\subsection{Summary and future directions}

In Drosophila, the simultaneous binding of the anterior determinant Bicoid (BCD) to the 5 '-cap associated factor d4EHP and the mRNA of the posterior patterning gene caudal (cad) leads to translational repression of the transcript. Because BCD is an evolutionary novelty in the lineage that lead to Drosophila, other insect species most likely follow alternative strategies to restrict cad mRNA to the posterior of the embryo. The establishment of an in vitro assay using EMSA and an in vivo sensor assay for BCD-mediated translational repression allowed me to identify elements in the cad 3'UTR of Drosophila and Haematopota pluvialis that mediate BCD-dependent translational repression. These elements show similarities in their predicted secondary structures.

The BCD-binding region of the Dm'cad 3'UTR co-localizes with a miR-308 target site and mutations in this region that abolish miRNA-binding suggest a miRNA-dependent function in BCD-mediated translational repression. Furthermore, BCD isoforms that lack the d4EHP-binding domain are able to mediate translational repression of sensors carrying the BCD-binding region. Taken together, these findings suggest that alternative mechanism(s) for the translational repression of cad mRNA are likely to exist in Drosophila and may also be present in other insect species.

The model of cad translational repression by $\mathrm{BCD}$ has become a paradigm for translational regulation, in which translational repression is mediated through competition of translational initiation factors by 4EHP (Cho et al., 2005). I suggest that other mechanisms may exist by which $\mathrm{BCD}$ can mediate cad translational repression in the absence of 4EHP activity. Thus, the model of cad translational repression by BCD has to be re-evaluated for a more complete picture. The following questions are posed by this work: How does this 4EHP-independent mechanism(s) function and how relevant is it for the development of the Drosophila embryo? How does miR-308 and/or other miRNAs contribute to translational control of cad? How important is the secondary structure of the BCD-binding element?

Generating an in vivo reporter that quantitatively senses BCD-dependent translational repression will be a useful tool in answering some of these questions. For instance it will be possible to introduce specific point mutations into BCD-binding regions that disrupt the secondary structure of the RNA and measure to what extent these mutations influence BCD-mediated repression. The role of miR-308 in cad translational 
repression may be approached by a rescue experiment. Providing a miRNA that contains complementary changes to the mutations introduced in the sensor 38FDm3'mut may result in a recovery of BCD-dependent translational repression of 38FDm3'mut, which can be tested using the in vivo reporter assay.

The findings of my PhD have also opened new directions for the investigation of the evolution of cad translational repression and early axis patterning in general. How conserved is 4EHP-independent regulation of cad among different insect species? Does this mechanism perhaps create a link between BCD-dependent anterior patterning in Drosophila and anterior patterning by Tc'MEX-3 and Tc'ZEN-2, the factors that regulate the cad homologue in Tribolium? Could it be a remnant of an ancestral mechanism that functioned before the co-option of BCD into translational control? It would be important to identify the factors that are involved in the 4EHP-independent mechanism of $B C D$ isoform $E$ and $F$ and to see, whether they play a role in translational regulation of cad in other insect species. This questions could be addressed by using co-immunoprecipitation of protein complexes formed with BCD isoform $\mathrm{F}$ and compare these to co-immunoprecipitated proteins of complexes formed with $B C D$ isoform $G$. Potential factor(s) that are present only in precipitates of $B C D$ isoform $\mathrm{F}$ may then be identified using mass spectroscopy. 


\section{Materials and Methods}

\subsection{Oligonucleotides}

\subsubsection{Oligonucleotides for cloning procedures}

Tab. 5.1: Oligonucleotides for cloning procedures; restriction sites are indicated in small letters.

\begin{tabular}{ll}
\hline Primer & Sequence (5' to 3') \\
\hline SV40-R01 & AGGGGGAGGTGGGGAGG \\
006-R_Ascl & ttggcgccGTGAACGTTAACCCTTATTAACC \\
007-F_EcoRI & ttgaattcTTCCCTCACAACTCATATGACCG \\
008-R_Ascl & tggcgcgccCAAAATAAAATCATTTATTCTTCAC \\
009-F_BamHI & cgggatccATGTGACACGACCATTCC \\
011-F_EcoRI & cggaattcTGACCACATTAAACGCAT \\
012-R_Ascl & aggcgcgccCTAAGTAATTTAAGTATAATTA \\
021-F_Ndel & ggaattccatatgCCACGTCGCACCCGCACC \\
022-R_BamHI & cgggatccCTACTAGGACTGGTCCTTGTGCTGATC \\
038-F_Kpnl & cgggtaccATGGCGCAACCGCCG \\
039-R_BamHI & gcggatcCATTGAAGCAGTAGGCAAACTGCG \\
043-F_Kpnl & gcggtaccATGGTGAGCAAGGGCGAG \\
044-R_Ascl & ggcgcgccCTAGACGTCCTCCACCTTG \\
046-F & TAACAAACTGCAATATTCCAGGTGCAACGGCCGCCAAGTCCT \\
047-R & CCATTCG \\
050-F_Eagl & ATTTTACTGATTGTATATTCCTGGTTTCGACACGCGCCAGAGT \\
058-F_Kpnl & ggcggccgGAAAATGCCCAAGCCAGAGGAG \\
059-F-Ascl & cgggtaccATGCCCAAGCCAGATGTCTTTCCCTCAG \\
060-R-Ascl & ggcgcgccGTAATACGACTCACTATAGGG \\
061-F-Avrll & ggcgcgccGGCATAACATGGTGACAT \\
062-R-Ascl & ccctaggaATGGTTTCGCACTACTACAAC \\
6MNLS-FW-Avrll & ccctaggaATGGTTTCGCACTACTACAAC \\
\hline & gccctaggAGGATCCCATCGATTTAAAGCTATGG \\
\hline &
\end{tabular}


5.1.2 Oligonucleotides for template generation of in vitro synthesized RNA probes

Tab. 5.2: Oligonucleotides for template generation of RNA probes PCR; restriction sites are indicated in small letters, T7 promoter region indicated by underlined letters

\begin{tabular}{ll}
\hline Primer name & Sequence (5' to 3') \\
\hline 015-R_Xbal & gctctagaGGCATAACATGGTGACAT \\
017-R_Ascl & tggcgcgccCATGATCAGATCAACATCAG \\
027-F_T7 & GTAATACGACTCACTATAGGGCGAATTGGAGCTCGGCCGTTGCACCTGG \\
028-R & GCGAATTCGAACCAGGAATATACAATCAC \\
033-F_T7 & GTAATACGACTCACTATAGGGCGAATGACCACATTAAACGCATTTG \\
034-F_T7 & GTAATACGACTCACTATAGGGCGAATATGCCTATGATCGGTTTTAC \\
035-F_T7 & GTAATACGACTCACTATAGGGCGAATTTGAATAATTTTAATTATAC \\
040-F_T7 & GTAATACGACTCACTATAGGGCGAGGCACCGGATTATCTAG \\
041-R & AAGCAACAAGAAGGCACATAAACGTTTGTAC \\
055-F_T7 & GTAATACGACTCACTATAGGGCGAACTAGATCATAATCAGCC \\
056-F_T7 & GTAATACGACTCACTATAGGGCGACTCTCTCTCTTCTTCCTCTCTC \\
$057-R$ & AGAAGAGGAGAGAGAAGGAGAGGAGAAAGAGAGAGAGGAAGAAGAG \\
\hline
\end{tabular}

\subsubsection{Oligonucleotides for generation of ds DNA probe}

Tab. 5.3: Oligonucleotides for generation of ds DNA probe; restriction sites are indicated in small letters.

\begin{tabular}{ll}
\hline Primer name & Sequence (5'->3') \\
\hline 031-F_EcoRI & gaattcGCTCTAATCCCCGAA \\
032-R_EcoRI & gaattcCGGGGATTAGAGC \\
\hline
\end{tabular}




\subsection{In vitro Methods}

\subsubsection{Cloning procedures}

\subsubsection{Cloning of protein expression construct}

pET16b-HisMBP-HD The fragment encoding the Bicoid homeodomain (spanning amino acids 97-163) was amplified from an embryonic cDNA library (given by $\mathrm{M}$. Kiparaki) by PCR using primers 021-F_Ndel and 022-R_BamHI and cloned into Ndel and $\mathrm{BamHI}$ in frame to the Maltose binding protein (MBP) of pET16b-HisMBPTev (courtesy of A. Economou). pET16b carries a N-terminal His-tag, followed by MBP, a cleavage site for the TEV protease and the Ndel and BamHI sites for directional cloning.

\subsubsection{Cloning of 3'UTRs used for EMSAs}

pSL-Dm3' The Drosophila caudal 3'UTR (spanning nt 1-862, numbering starts at first nucleotide after the stop codon, followed by $40 \mathrm{nt}$ of genomic sequence) was amplified from Drosophila genomic DNA by PCR using primers 009-F_BamHI and 006-R_Ascl and cloned as an BamHI/blunt fragment into BamHI/EcoRV sites of pBluescript II KS(+) (Stratagene), creating pBS-Dm3'. The Drosophila caudal 3'UTR was cloned from pBSDm3' as a Spel/HindIII fragment into pSLfa1180fa (Horn and Wimmer, 2000).

pBS-BRE_Styl/Pstl The BRE_Styl-Pstl fragment (spanning nt 164-512, numbering starts at first nucleotide after the stop codon) from the Drosophila caudal 3'UTR was cloned into Xbal and Pstl sites of pBluescript II KS(+).

pBS-BRE_164-339 A Notl/Xhol fragment from pBS-BRE_Styl/Pstl was isolated, digested with Alul to create the BRE_164-339 fragment (spanning nt 164-339, numbering starts at first nucleotide after the stop codon) and cloned into Notl/EcoRV sites of pBluescript II KS(+).

pSL-Hp3' $\Delta$ EcoRI The Haematopota caudal 3'UTR was amplified by PCR from plasmid \#436 (courtesy of U. Schmidt-Ott) using primers 011-F_EcoRI and 012-R_Ascl and 
cloned as a blunt/Ascl fragment into EcoRV/Ascl sites of pBS-HAE-3xP3-DsRed -attBSV40. The 3'UTR was excised as a BgllI/Nrul fragment, inserted into BamHI/EcoRV sites of pBluescript II KS(+), creating pBS-Hp3', and then subcloned into pSLfa1180fa as a Spel/HindIII fragment. Next, an EcoRI fragment was excised and the plasmid religated.

pSL-Tc3' The Tribolium caudal 3'UTR of splice variant A (spanning nt 751-1177, numbering starts at first nucleotide after the stop codon, followed by 47 nt of genomic sequence) was amplified from Tribolium genomic DNA by PCR using primers 007F_EcoRI and 008-R_Ascl and cloned as an EcoRI/blunt fragment into EcoRI/EcoRV sites of pBluescript II KS(+). Then it was cloned into pSLfa1180fa as an EcoRI/HindIII fragment.

pSL-SV40 The SV40 3'UTR was excised from pJB26 (Brennecke et al., 2003) as a HindIII/Xbal fragment and cloned into HindIII/Xbal sites of pBluescript II KS(+), creating pBS-SV40, and then cloned as an Eagl/HindIII fragment into pSLfa1180fa .

pBS-Adh3' $\triangle \mathrm{Pacl} / \mathrm{EcoRV}$ The Adh 3'UTR was excised from ract-vector (Swevers et al. 1996) as a Xbal fragment and cloned into the Xbal site of pBluescript II KS(+), with the 5 ' end towards the T7 promoter region. Excision of a Pacl/EcoRV fragment and religation results in $\mathrm{pBS}$-Adh3' $\triangle \mathrm{Pacl} / \mathrm{EcoRV}$. 


\subsubsection{RNA probes}

\subsubsection{Template generation}

BRE_164-339 pBS-BRE_164-339 was linearized with HindIII and RNA transcribed with T7 RNA polymerase, resulting in a RNA fragment of 214 nt including T7 promoter sequence.

BRE_257-319 A DNA fragment containing nt 257-319 from the Drosophila caudal 3'UTR (numbering starts at first nucleotide after the stop codon) was amplified by PCR using pBS-Dm3' as template and primers 027-F_T7 and 028-R, RNA transcribed with T7 RNA polymerase, resulting in a RNA fragment of 84 nt including T7 promoter sequence.

H1 A DNA fragment containing nt 1-52 of the Haematopota caudal 3'UTR (numbering starts at first nucleotide after the stop codon) was amplified by PCR using pSLHp3' $\Delta$ EcoRI as template and primers 033-F_T7 and 015-R_Xbal, RNA transcribed with T7 RNA polymerase, resulting in a RNA fragment of 67 nt including T7 promoter sequence.

H2 A DNA fragment containing nt 52-89 of the Haematopota caudal 3'UTR (numbering starts at first nucleotide after the stop codon) was amplified by PCR using pSLHp3' $\Delta$ EcoRI as template and primers 034-F_T7 and 017-R_Ascl, RNA transcribed with T7 RNA polymerase, resulting in a RNA fragment of 59 nt including T7 promoter sequence.

H3 A DNA fragment containing nt 88-126 of the Haematopota caudal 3'UTR (numbering starts at first nucleotide after the stop codon) was amplified by PCR using pSL-Hp3' $\Delta$ EcoRI as template and primers 035-F_T7 and 012-R_Ascl, RNA transcribed with T7 RNA polymerase, resulting in a RNA fragment of 51 nt including T7 promoter sequence.

H2/H3 A DNA fragment containing nt 52-126 of the Haematopota caudal 3'UTR (numbering starts at first nucleotide after the stop codon) was amplified by PCR using pSL-Hp3' $\Delta E c o R I$ as template and primers 034-F_T7 and 012-R_Ascl, RNA transcribed with T7 RNA polymerase, resulting in a RNA fragment of $96 \mathrm{nt}$ including T7 promoter sequence. 
Tc_245-307 A DNA fragment containing nt 245-307 of the Tribolium caudal 3'UTR (numbering starts at first nucleotide after the stop codon) was amplified by PCR using pSL-Tc3' as template and primers 040-F_T7 and 041-R, RNA transcribed with T7 RNA polymerase, resulting in a RNA fragment of 75 nt including $T 7$ promoter sequence.

shSV40 A DNA fragment containing a 72 nt fragment of the SV40 3'UTR was amplified by PCR using pSL-SV40 as template and primers 055-F_T7 and SV40-R01, RNA transcribed with T7 RNA polymerase, resulting in a RNA fragment of $79 \mathrm{nt}$ including T7 promoter sequence.

Adh3' The pBS-Adh3' $\triangle$ Pacl/EcoRV plasmid was linearized using Hindlll and RNA transcribed with T7 RNA polymerase, resulting in a RNA fragment of 184 nt including $\mathrm{T} 7$ promoter sequence.

CU58 A DNA fragment containing a randomized $C / T$ sequence was generated by PCR using primers 056-F_T7 and 057-R that are complementary at their 3'-ends. Primer 056-F_T7 includes the T7 promoter sequence. RNA was transcribed with T7 RNA polymerase, resulting in a RNA fragment of $58 \mathrm{nt}$.

\subsubsection{In vitro transcription and radioisotope-labeling of RNA probes}

For all probes RNA was in vitro transcribed using the MEGAscript T7 kit (Ambion) according to manufacturers instructions. After synthesis, the template DNA was treated with TurboDNase, the RNA Phenol/Chloroform extracted and precipitated in Ammonioumacetate and 2-propanol. 50 pmole of RNA were resuspended in nucleasefree $\mathrm{H}_{2} \mathrm{O}$ and dephosphorylated using 10 units of Shrimp Alkaline Phosphate (Roche) by incubating for $1 \mathrm{~h}$ at $37^{\circ} \mathrm{C}$ and heat inactivating for $20 \mathrm{~min}$ at $65^{\circ} \mathrm{C}$. Next the RNA was precipitated in Sodiumacetate and $\mathrm{EtOH}$, resuspended in nuclease-free $\mathrm{H}_{2} \mathrm{O}$ and concentration and purity were determined spectrophotometrically. The RNA was 5' end labeled using 20 units of T4 Polynucleotide Kinase (New England Biolabs) and $5 \mu{ }^{32} \mathrm{P}$ $\gamma$ ATP $(10 \mu \mathrm{Ci} / \mu \mathrm{l},>3000 \mathrm{Ci} / \mathrm{mmol})$, by incubating for $1-2 \mathrm{~h}$ at $37^{\circ} \mathrm{C}$ and purifying over a Sephadex G-50 column (GE Healthcare). 


\subsubsection{Radioisotope-labeling of dsDNA oligonucelotides}

Radioisotope-labeled dsDNA oligonucleotides containing the consensus Bcd target site (5'-TAATCC-3') (Baird-Titus et al., 2006) were generated as follows: Oligonucleotides $031-\mathrm{F}$ and $032-\mathrm{R}$ ( 5 pmole/ $\mu \mathrm{l}$ each) were mixed for annealing in an equimolar ratio in TE with $50 \mathrm{mM} \mathrm{NaCl}$, incubated for 2 min at $95^{\circ}$ using a heat block and let to cool down to RT in the heat block. 20 pmole of the dsDNA were radioisotope-labeled using 2.5 units of the Large Klenow fragment (NEB), $3 \mu \mathrm{l}$ of ${ }^{32} \mathrm{P}-\gamma \mathrm{ATP}(10 \mu \mathrm{Ci} / \mu \mathrm{l},>3000$ $\mathrm{Ci} / \mathrm{mmol}$ ) in a total volume of $100 \mu \mathrm{l}$, incubated for $10 \mathrm{~min}$ at RT and purified over a Sephadex G-50 column.

\subsubsection{Protein purification}

\subsubsection{Purification of HisMBP-HD fusion protein}

For the purification of the homeodomain fused to MBP, E.coli strain BL21(DE3)pLysS was transformed with pET16b-HisMBP-HD and grown in LB medium containing 100 $\mu \mathrm{g} / \mathrm{ml}$ ampicilin and $35 \mu \mathrm{g} / \mathrm{ml}$ chloramphenicol. $500 \mathrm{ml}$ of LB medium containing 100 $\mu \mathrm{g} / \mathrm{ml}$ ampicilin were inoculated to an $\mathrm{OD}_{600}=0.1$ using an overnight culture and grown at $37^{\circ} \mathrm{C}$ with vigorous shaking $(225 \mathrm{rpm})$ to an $\mathrm{OD}_{600}=0.4$. Expression of the recombinant protein was induced by addition of IPTG to a final concentration of 0.1 $\mathrm{mM}$. The cells were harvested, resuspended in $15 \mathrm{ml} \mathrm{LEW}$ buffer $\left(50 \mathrm{mM} \mathrm{NaH} \mathrm{PO}_{4}\right.$, $300 \mathrm{mM} \mathrm{NaCl}, \mathrm{pH}$ 8.0) disrupted by sonication. All subsequent procedures were carried out at $4^{\circ} \mathrm{C}$. The crude lysate was centrifuged for $30 \mathrm{~min}$ at $13000 \mathrm{rpm}$ at $4^{\circ} \mathrm{C}$ and the supernatant transferred to a clean tube, containing $1.5 \mathrm{~g}$ Protino Ni-TED Resin. The lysate-resin mixture was incubated for $15 \mathrm{~min}$, transferred to an empty chromatography column that was blocked with some glass wool and left to settle by gravity-flow. The column was washed with $25 \mathrm{ml}$ of LEW buffer and the HisMBP-HD fusion protein eluted in 5 fractions of $5 \mathrm{ml}$ Elution buffer $\left(50 \mathrm{mM} \mathrm{NaH}_{2} \mathrm{PO}_{4}, 300 \mathrm{mM} \mathrm{NaCl}, 250 \mathrm{mM}\right.$ imidazole, $\mathrm{pH}$ 8.0). The purity of the recombinant protein was analyzed by SDS-PAGE and the first two fractions combined. The protein solution was concentrated by dialysis in $1 \mathrm{~L}$ of dialysis buffer (20 mM Tris- $\mathrm{Cl}(\mathrm{pH} 7.5), 50 \mathrm{mM} \mathrm{KCl}, 0.5 \mathrm{mM}$ EDTA, $10 \%$ glycerol) for $3 \mathrm{~h}$ at $4^{\circ} \mathrm{C}$ and a second dialysis in $500 \mathrm{ml}$ of storage buffer (20 mM Tris-Cl (pH 7.5), $50 \mathrm{mM} \mathrm{KCl}, 0.5 \mathrm{mM}$ EDTA, $50 \%$ glycerol) overnight at $4^{\circ} \mathrm{C}$. The final volume of the protein solution was $2.5 \mathrm{ml}$, it was supplemented with DTT to a final concentration of $1 \mathrm{mM}$, divided into $250 \mu \mathrm{l}$ aliquots and stored at $-80^{\circ} \mathrm{C}$. 


\subsubsection{Purification of GST-HD fusion protein}

For the purification of the homeodomian fused to GST, E.coli strain BL21(DE3)pLysS was transformed with PTA67A (Baird-Titus et al., 2006). PTA67A is a pET41 expression vector carrying the Bicoid homeodomain (spanning amino acids 97-163) fused C-terminally to Glutathione-S-tranferase (GST), followed by a Tev protease cleavage site. Cells were grown in LB medium containing $50 \mu \mathrm{g} / \mathrm{ml}$ kanamycin and 35 $\mu \mathrm{g} / \mathrm{ml}$ chloramphenicol. $1 \mathrm{~L}$ of $\mathrm{LB}$ medium containing $50 \mu \mathrm{g} / \mathrm{ml}$ kanamycin were inoculated to an $\mathrm{OD}_{600}=0.1$ using an overnight culture and grown at $37^{\circ} \mathrm{C}$ with vigorous shaking $(225 \mathrm{rpm})$ to an $\mathrm{OD}_{600}=0.4$. Expression of the recombinant protein was induced by addition of IPTG to a final concentration of $0.2 \mathrm{mM}$. The cells were harvested, resuspended in $50 \mathrm{ml}$ ice-cold PBS and disrupted by sonication. The crude lysate was incubated for $30 \mathrm{~min}$ with gentle shaking at $4^{\circ} \mathrm{C}$ with $1 \%$ Triton-X 100 , centrifuged for $20 \mathrm{~min}$ at $13000 \mathrm{rpm}$ at $4^{\circ} \mathrm{C}$ and the supernatant transferred into a fresh tube. $70 \mathrm{mg}$ of Glutathione agarose resin (soaked overnight at $4^{\circ} \mathrm{C}$ in $15 \mathrm{ml} P B S$ ) were added as a slurry to the supernatant and incubated with gentle shaking for $30 \mathrm{~min}$ at RT. All subsequent procedures were carried out at $4^{\circ} \mathrm{C}$. The lysate-resin mixture was transferred to an empty chromatography column that was blocked with glass wool and left to settle by gravity-flow. The column was washed with $30 \mathrm{ml}$ of ice cold PBS and the GST-HD recombinant protein eluted in 5 fractions of $1 \mathrm{ml} \mathrm{GST} \mathrm{Elution} \mathrm{buffer} \mathrm{(10}$ $\mathrm{mM}$ reduced Glutathione, $50 \mathrm{mM}$ Tris- $\mathrm{Cl} \mathrm{pH} 8.0$ ). The purity of the recombinant protein was analyzed by SDS-PAGE and the first 4 fractions combined. The protein solution was concentrated by dialysis in $1 \mathrm{~L}$ of dialysis buffer $(20 \mathrm{mM}$ Tris- $\mathrm{Cl}(\mathrm{pH} 7.5), 50 \mathrm{mM}$ $\mathrm{KCl}, 0.5 \mathrm{mM}$ EDTA, $10 \%$ glycerol) for $4 \mathrm{~h}$ and a second dialysis in $500 \mathrm{ml}$ of storage buffer (20 mM Tris-Cl (pH 7.5), $50 \mathrm{mM} \mathrm{KCl,} 0.5 \mathrm{mM}$ EDTA, 50 \% glycerol) overnight. The final volume of the protein solution was $1 \mathrm{ml}$, it was supplemented with DTT to a final concentration of $1 \mathrm{mM}$, divided into $250 \mu \mathrm{l}$ aliquots and stored at $-20^{\circ} \mathrm{C}$ or $-80^{\circ} \mathrm{C}$.

\subsubsection{Tev-protease digest of HD}

The homeodomain was liberated from its fusion protein MBP or GST as follows: $100 \mu \mathrm{g}$ of HisMBP-HD or GST-HD fusion protein were incubated with 50 units of AcTEVProtease (Invitrogen) for $6 \mathrm{~h}$ at RT or overnight at $4^{\circ} \mathrm{C}$ in a final volume of $150 \mu \mathrm{l}$. The digest was tested by electrophoresis on a polyacrylamide gel and coumassie staining. 
The homeodomain was then directly used for in vitro binding to RNA and EMSA experiments.

\subsubsection{Electrophoretic Mobility Shift Assays (EMSAs)}

\subsubsection{RNA EMSAs}

In binding experiments, different concentrations of HisMBP-HD or GST-HD or liberated homeodomain were incubated with 20 - 300 fmole of radioisotope-labeled RNA probes (reaction volume $10 \mu \mathrm{l}$ ) for $10 \mathrm{~min}$ on ice in binding buffer (final concentrations during binding reaction $10 \mathrm{mM}$ HEPES $\mathrm{pH} 7.5,100 \mathrm{mM} \mathrm{KCl}, 1 \mathrm{mM} \mathrm{MgCl} 2,0.5 \mathrm{mM}$ EDTA, 1 $\mathrm{mM}$ DTT, $0.5 \mu \mathrm{g} / \mu \mathrm{l}$ yeast tRNA, 10\% glycerol). Prior to binidng, the radioisotopelabeled probes were incubated in binding buffer (without tRNAs) at $70^{\circ} \mathrm{C}$ for 5 min and chilled on ice for $5 \mathrm{~min}$. The binding reactions were loaded on $0.5 x$ TBE polyacrylamide gels electrophorized for $5-6 \mathrm{~h}$ at $10 \mathrm{~V} / \mathrm{cm}$ at $4^{\circ} \mathrm{C}$. The radioactivity was visualized using phosphoimager.

In competition experiments, constant amounts of radioisotope-labeled RNA probes were pre-incubated at $70^{\circ} \mathrm{C}$ for $5 \mathrm{~min}$ followed by $5 \mathrm{~min}$ on ice. HisMBP-HD, GST-HD or liberated homeodomain were then added and incubated (reaction volume $10 \mu \mathrm{l}$ ) for $10 \mathrm{~min}$ on ice in binding buffer. $1 \mu \mathrm{l}$ of cold competitor, pre-incubated at $70^{\circ} \mathrm{C}$ for $5 \mathrm{~min}$ followed by 5 min on ice, diluted in binding buffer in different amounts were added to the binding reaction and incubated for $10 \mathrm{~min}$ on ice. The reactions were then loaded on $0.5 \mathrm{x}$ TBE polyacrylamide gels electrophorized for $5-6 \mathrm{~h}$ at $10 \mathrm{~V} / \mathrm{cm}$ at $4^{\circ} \mathrm{C}$. The radioactivity was visualized using phosphoimager.

\subsubsection{DNA EMSAS}

Different concentrations of HisMBP-HD or GST-HD or liberated HD were incubated with 2 pmole of radioisotope-labeled dsOligo (reaction volume $10 \mu \mathrm{l}$ ) for $10 \mathrm{~min}$ on ice in binding buffer (same as in 5.2.5.1) and electrophorized on a 0.5x TBE polyacrylamide gel for $5-6 \mathrm{~h}$ at $10 \mathrm{~V} / \mathrm{cm}$ at $4^{\circ} \mathrm{C}$. The radioactivity was visualized using phosphoimager. 


\subsection{In vivo Methods}

\subsubsection{Cloning procedures of in vivo methods}

\subsubsection{Cloning of sensor constructs}

pJBattP-tub $\alpha$-1-nGFP-attP-SV40 The attP recombination site was cloned from pTAattP (Groth et al., 2003) as a $326 \mathrm{nt} \mathrm{Xbal} \mathrm{and} \mathrm{BamHI} \mathrm{fragment} \mathrm{into} \mathrm{Nhel} \mathrm{and} \mathrm{BamHI}$ sites of pSLfa1180fa, resulting in pSL-attP>. The EGFP followed by six Myc-tag repeats and a NLS was cloned as a Spel and Xbal fragment from GN1 (courtesy of G.

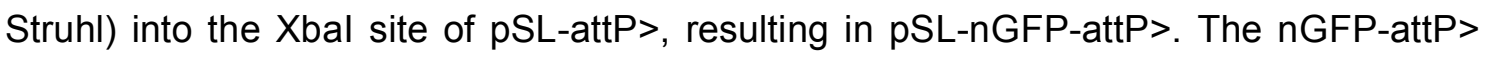
fragment was cloned as a Kpnl and Sall fragment into Kpnl and Xhol sites of pJB26 (Brennecke et al., 2003), which removes the EGFP and places the nGFP in frame with the ORF that initiates in the tub $\alpha-1$ promoter fragment.

pHAE-3xP3-DsRed-attB-SV40 The MCS of pSLfa1180fa was excised as a Ascl fragment and inserted into BssHII sites of pBluescript II $\mathrm{KS}(+)$, placing the HindIII site next to the M13 -20 primer site and the EcoRI site next to the M13 reverse primer binding site. The plasmid was digested by Narl, filled-in and religated, creating in a new Ascl site (plasmid pHAE). The attB recombination site was amplified from pTA-attB (Groth et al., 2003) using primers pTA-attB-F2 and BamHI-pTA-attB-R and cloned as a blunt and BamHI fragment into Smal and BamHI sites of pHAE resulting in pHAEattB>. The SV40 3'UTR was cloned as Xbal and HindlII fragment from pJB26 into Spel and HindlII sites of pHAE-attB>, resulting in pHAE-attB>-SV40. 3xP3-DsRed was cloned as a Notl-blunted/EcoRI fragment from pMi-3xP3-DsRed-SV40 (Pavlopoulos and Averof, 2005) into Smal and EcoRI sites of pHAE-attB>-SV40.

pHAE-3xP3-DsRed-attB-Dm3'-SV40 The Dm3' was amplified by PCR using genomic DNA as template and primers 009-F_BamHI and 006-R_Ascl, the PCR product digested with Ascl and cloned into EcoRV/Ascl sites. 
pHAE-3xP3-DsRed-attB-Hp3'-SV40 The Hp3' was amplified by PCR using pSL-Hp3' as template and primers 011-F_EcoRI and 012-R_Ascl, the PCR product digested with Ascl and cloned into EcoRV/Ascl sites.

pHAE-3xP3-DsRed-attB-Tc3'-SV40 The Tc3' was cloned as a Fselblunted/Ascl fragment from vector \#538 (courtesy of Schmidt-Ott) into Nrul/Ascl sites.

pit-GFP The tuba-1-nGFP fragment was cloned as a HindlII/Notl-blunted fragment from pJBattP-tuba-1-nGFP-attP-SV40 into HindIII/Xhol-blunted sites of pHAE. Then it was removed as a HindIII and Bglll fragment and inserted into HindIII and BamHI sites of piB-GFP (Bateman et al. 2006), replacing the hsp70 promoter and GFP sequences. The unique Mlul and Ascl sites following the ORF of the nGFP were used for cloning of 3'UTR fragments.

pit-GFP-Dm3' The Dm3' was inserted as a BssHI fragment from pBS-Dm3' into Ascl.

pit-GFP-SV40 The SV40 was inserted as a BssHI fragment from pBS-SV40 into Ascl.

piB-TdnGFP The tub $\alpha-1$ promoter was cloned as a EcoRV and Kpnl fragment from pJB26 into EcoRV and Kpnl sites of pHAE, resulting in pHAE-tub $\alpha-1$. A destabilized EGFP (BD Biosciences Clontech) fused C-terminally to 6 Myc-tags and a NLS (dnGFP) was amplified by PCR using primers 043-F_Kpnl and 044-R_Ascl from pd2EGFP6MNLS (given by P.Piwko) and cloned into Kpnl and Ascl sites of pHAEtuba-1. The unique Kpnl site in piB-GFP, which is lying outsite of the attB recombination sites, was destroyed by Kpnl digest, removal 3' overhangs by Klenow and religation, resulting in piB-GFP $\Delta \mathrm{Kpnl}$. The tub $\alpha-1-d n G F P$ was then cloned as a Bglll and Xhol fragment into BamHI and Sall sites of piB-GFP $\Delta K p n l$, replacing the hsp70 promoter and GFP sequences, resulting in piB-TdnGFP. Due to inconsistencies in diagnostic digests, the tub $\alpha-1$ promoter was replaced by excision of a HindlII and Kpnl fragment and insertion of a HindIII and Kpnl fragment from pit-GFP. Due to a point mutation, the NLS was replaced by a 6xMyc-NLS fragment, which was amplified 
by PCR using primers 6MNLS-FW-Avrll and 044-R_Ascl and cloned into Avrll and Ascl sites of piB-TdnGFP. The unique Ascl and Mlul sites following the ORF of dnGFP were used for cloning of 3'UTR fragments.

piB-TdnGFP-Dm3' The Dm3'UTR was inserted as a Mlul/Ascl fragment from pSL-Dm3' into Ascl.

piB-TdnGFP-Hp3' The Hp3' UTR was inserted as an Ascl fragment from pSLHp3' $\Delta$ EcoRI into Ascl.

piB-TdnGFP-Tc3' The Tc3' UTR was inserted as an Ascl fragment from pSLTc3' into Ascl.

piB-TdnGFP-SV40 The SV40 UTR was inserted as an Ascl fragment from pSLSV40 into the Mlul.

piB-TdnGFP-Dm3'mut The seed sequence of the predicted mir-308 target site was mutagenised by PCR using pBS-Dm3' as template and primers 046-F and 047-R, which are lying back-to-back, generating the full plasmid including $4 \mathrm{nt}$ changes (mutated mir-308 target sequence TTGCAgtTTGTTAATTTTacTGATT, with changed nt indicated in small letters). The parental methylated nonmutated template DNA was digested with Dpnl and the resulting linear mutagenised plasmid ligated into a circular form. The mutagenised Dm3'mut was then cloned as a Notl/HindIII fragment into Eagl/HindIII sites of pSLfa1180fa, creatin pSL-Dm3'mut. The mutated Dm3'UTR was inserted as a Ascl fragment from pSL-Dm3'mut.

piB-TdnGFP-BRE_257 The BRE_257-319 fragment was amplified by PCR using primers 027-F_T7 and 028-R and cloned into blunted Ascl site of piBTdnGFP-SV40.

piB-TdnGFP-H1 The $\mathrm{H} 1$ fragment was amplified by PCR using primers 033F_T7 and 015-R_Xbal and cloned into blunted Ascl site of piB-TdnGFP-SV40.

piB-TdnGFP-H2 The $\mathrm{H} 2$ fragment was amplified by PCR using primers 034F_T7 and 017-R_Ascl and cloned into blunted Ascl site of piB-TdnGFP-SV40.

piB-TdnGFP-H3 The H3 fragment was amplified by PCR using primers 035F_T7 and 012-R_Ascl and cloned into blunted Ascl site of piB-TdnGFP-SV40. 
piB-HdnGFP The maternal hbP1 promoter was cloned from $\mathrm{pChbP} 1 \Delta$ Xbal (Wimmer et al., 2000) as a Xbal and BamHI fragment into pSLfa1180fa. Next it was excised as an Avrll/Xbal fragment and inserted into Nhel and Xbal sites of pSLfa1180fa. The hbP1 promoter was then excised as a Sphl and Kpnl fragment and inserted into Sphl and Kpnl sites of piB-TdnGFP, replacing the tub $\alpha-1$ promoter. The unique Ascl and Mlul sites following the ORF of dnGFP was used for cloning of 3'UTR fragments.

piB-HdnGFP-Dm3' The Dm3' was inserted as a Mlul/Ascl fragment from pSLDm3' into Ascl.

piB-HdnGFP-SV40 The SV40 was inserted as an Ascl fragment from pSLSV40 into Ascl.

piB-HR The HIST1H2BJ-mRFPruby was excised from pAc5.1-HIST1H2BJ-mRFPruby (Müller-Taubenberger et al., 2006) as a Kpnl/Mlul and inserted into piB-HdnGFP, replacing dnGFP. The unique Mlul site which follows the ORF of HIST1H2BJmRFPruby can be used for cloning of 3'UTR fragments.

piB-HR-Dm3' The Dm3' UTR was inserted as a Mlul/Ascl fragment from pSLDm3'.

piB-HR-SV40 The SV40 was inserted as an Ascl fragment from pSL-SV40 into Ascl.

piB-HGFPCAD The cad ORF was amplified from an embryonic cDNA library using primers 061-F_Avrll and 062-R_Ascl and cloned into Avrll and Ascl sites of piB-HDN, replacing the 6xMyc-NLS and fusing the cad ORF C-terminally in frame to the d2EGFP. The unique Ascl site following the ORF of d2EGFP-CAD fusion was used for cloning of 3'UTR fragments. The Dm3' UTR was inserted as a Mlul/Ascl fragment from pSL-Dm3'.

piB-HGFPCAD-Dm3' The Dm3' UTR was inserted as a Mlul/Ascl fragment from pSL-Dm3'. 


\subsubsection{Cloning UAS constructs}

pUASp2-6M A BamHI fragment from GN1 containg 6xMyc-tag was cloned into BamHI/Bglll of pSLfa1180fa, destroying the C-terminal BamHI site. Next it was cloned as a BamHI/Xbal fragment into pUASp2 (Rørth, 1998).

pUASp2-BcdG-6M The ORF of Bcd isoform G (spanning 494 aa without stop codon) was amplified from an embryonic cDNA library by PCR using primers 038-F_Kpnl and 039-R_BamHI and cloned into pGEM T easy. BcdG was then excised as a Notl/BamHI fragment and cloned N-terminally in frame to the 6xMyc-tag of pUASp2-6M.

pUASp2-BcdD-6M Bcd isoform D was ordered as a cDNA pOT2-LD36304 from the Drosophila Genomics Resource Center, Bloomington, IN. The ORF (spanning 489 aa without stop codon) was amplified by PCR using pOT2-LD36304 as template, primers 038-F_Kpnl and 039-F_BamHI and cloned into pGEM T easy. BcdD was then excised as a $\mathrm{Kpnl} / \mathrm{BamHI}$ fragment and cloned $\mathrm{N}$-terminally in frame to the 6xMyc-tag into pUASp2-BcdG-6M, replacing BcdG.

pUASp2-BcdE-6M Bcd isoform E (spanning 418 aa without stop codon) was amplified from an embryonic cDNA library by PCR using primers 058-F_Kpnl and 039-R_BamHI and cloned into pGEM T easy. BcdE was cloned as a Notl/BamHI fragment $\mathrm{N}$ terminally in frame to the 6xMyc-tag into pUASp2-BcdG-6M, replacing BcdG.

pUASp2-BcdF-6M Bcd isoform $F$ (spanning 413 aa without stop codon) was amplified from a cDNA library by PCR using primers 050-F_Eagl and 039-R_BamHI and cloned into pGEM T easy. BcdF was cloned as a Notl/BamHI fragment $\mathrm{N}$-terminally in frame to the 6xMyc-tag into pUASp2-BcdG-6M, replacing BcdG.

pUASp2-BcdA-6M Bcd isoform A (spanning 149 aa without stop codon) was amplified from an embryonic cDNA library by PCR using primers 038-F_Kpnl and 039-R_BamHI and cloned into pGEM T easy. BcdA was cloned as a Notl/BamHI fragment $\mathrm{N}$ terminally in frame to the 6xMyc-tag into pUASp2-BcdG-6M, replacing BcdG. 


\subsubsection{Drosophila melanogaster handling and husbandry}

Drosophila melanogaster cultures were kept at $25^{\circ} \mathrm{C}$ unless indicated otherwise and handled according to protocols described in Ashburner (1989). Food medium contained a mixture of yeast, sugar, agar, corn flour and Nipogen. Embryos were collected using cages and cherry juice plates (cherry juice, 3\% agar, Nipogen), adding live yeast paste to enhance egg laying. General fly stocks used for transgensis and genetic crosses are listed in Tab. 5.3.

Tab. 5.3: : General Fly stocks.

\begin{tabular}{|c|c|}
\hline name & genotype \\
\hline yw & $\mathrm{Df}(1) \mathrm{yw} \mathrm{w}^{6 / \mathrm{c} 23}$ \\
\hline $38 \mathrm{~F}$ & yw; P\{attP.w $\left.{ }^{+} . a t t P\right\} J B 38 F$ \\
\hline \multirow[t]{2}{*}{$53 \mathrm{~F}$} & $\mathrm{yw} ; \mathrm{P}\left\{\mathrm{attP} \cdot \mathrm{w}^{+} \cdot \mathrm{attP}\right\} \mathrm{JB} 53 \mathrm{~F}$ \\
\hline & nos-GAL4:VP16 \\
\hline yw;TM3Sb/TM6B & $\mathrm{Df}(1) \mathrm{yw}^{67 \mathrm{c} 23} ; \mathrm{TM} 3, \mathrm{Sb}, \mathrm{e} / \mathrm{TM} 6 \mathrm{~B}, \mathrm{Hu}, \mathrm{e}$ \\
\hline \multirow[t]{3}{*}{ yw;Sco/CyO } & $\mathrm{Df}(1) \mathrm{yw}^{67 \mathrm{c} 23} ;$ Sco $/ \mathrm{CyO}$ \\
\hline & $\mathrm{FM} 7 / \mathrm{w}^{\text {lethal; }} \mathrm{CyO} / \mathrm{Sp}$ \\
\hline & yw; CyO/if; MKRS/TM6B \\
\hline
\end{tabular}

\subsubsection{Transgenesis}

Microinjections of embryos were performed by loannis Livadaras using a NARISHIGE Microinjector IM - 300. Glass needles were pulled using SUTTER Flaming/ Brown Micropipette Puller P- 97. Injected embryos were covered with halocarbon oil and hatching larvae transferred to food vials. 


\subsubsection{1 $\varphi$ C31 integrase mediated recombination system using a single attP landing site (strategy 1)}

Capped mRNA of the phiC31 integrase was produced as follows: pET11[ $\varphi$ C 31 integrase-pA] (Groth et al., 2003) was linearized by BamHI digest and used as template for in vitro transcription with the mMESSAGE mMACHINE T7 kit (Ambion). The template DNA was treated with TurboDNase, the RNA Phenol/Chloroform extracted, precipitated in 2-propanol and resuspended in nuclease-free $\mathrm{H}_{2} \mathrm{O}$. An injection mixture containing the attB donor plasmid at high purity $(200-400 \mathrm{ng} / \mathrm{\mu l})$, capped mRNA of $\varphi \mathrm{C} 31$-integrase $(600-800 \mathrm{ng} / \mu \mathrm{l})$ and injection buffer $(10 \mathrm{mM}$ Tris-Cl $\mathrm{pH} 7.5,0.1 \mathrm{mM}$ EDTA, $10 \mathrm{mM} \mathrm{NaCl}$ ) was injected into embryos of line pJBattP-M44 line. G0 adults were backcrossed to $\mathrm{yw} ; \mathrm{Sco} / \mathrm{CyO}$ and the $\mathrm{G} 1$ progeny screened as larvae for 3xP3-DsRed expression. 3xP3-DsRed G1 individuals carrying the CyO balancer chromosome were backcrossed to $\mathrm{yw} ; \mathrm{Sco} / \mathrm{CyO}$ and balanced over CyO.

\subsubsection{Recombinase-mediated exchange cassette (RMCE) using $\varphi \mathrm{C} 31$ integrase (strategy 2)}

The nos- $\varphi$ C 31 -int;38F provides the $\varphi \mathrm{C} 31$ integrase maternally and was created by crossing line nos- $\varphi \mathrm{C} 31$-int (Bischof et al., 2007) to line 38F and making the progeny homozygous. An injection mixture containing the RMCE attB donor plasmid at high purity (200-400 $\mathrm{ng} / \mu \mathrm{l})$ and injection buffer (10 mM Tris-Cl pH 7.5, $0.1 \mathrm{mM}$ EDTA, 10 $\mathrm{mM} \mathrm{NaCl}$ ) was injected into embryos of the line nos- $\varphi \mathrm{C} 31$-int;38F. G0 adults were backcrossed to $\mathrm{yw}$;Sco/CyO and the G1 progeny screened as adults for loss of miniwhite phenotype. $\mathrm{G} 1 \mathrm{w}^{-}$males that originated from a $\mathrm{G} 0$ male were crossed to $\mathrm{yw}$;Sco/CyO virgins and the G2 progeny kept as a balanced stock over CyO. G1 wmales that originated from a G0 female, inherited the nos- $\varphi$ C31-int chromosome and were crossed as single $\mathrm{G} 1 \mathrm{w}^{-}$males to $\mathrm{yw} ; \mathrm{Sco} / \mathrm{CyO}$ virgins, a single $\mathrm{G} 2 \mathrm{w}^{-}$males to virgins of $\mathrm{yw}$;Sco/CyO. The G3 progeny was kept as a balanced stock over CyO.

\subsubsection{P-element based transgenesis}

Injection mixtures containing P-element plasmids at high purity $(300-400 \mathrm{ng} / \mu \mathrm{l}), \Delta 2-3$ helper plasmid (100-200 $\mathrm{ng} / \mu \mathrm{l})$ and injection buffer $(10 \mathrm{mM}$ Tris-Cl $\mathrm{pH} 7.5,0.1 \mathrm{mM}$ EDTA, $10 \mathrm{mM} \mathrm{NaCl}$ ) were injected into yw embryos. G0 adults were backcrossed to yw and the $\mathrm{G} 1$ progeny screened for $\mathrm{w}^{+}$individuals. $\mathrm{G} 1 \mathrm{w}^{+}$individuals were backcrossed 
to $\mathrm{yw}$ and the segregation of the $\mathrm{w}^{+}$analyzed in the $\mathrm{G} 2$ progeny to identify single insertions. Chromosome mapping of independent single insertions was done by segregation analysis as follows, G2 individuals were crossed to $2^{\text {nd }}$ chromosome balancer stock yw; Sco/CyO and $3^{\text {rd }}$ chromosome balancer stock yw; TM3Sb/TM6B. G3 individuals were backcrossed to the same respective balancer stock and the insertion mapped to the chromosome that does not give rise to flies with w phenotype in the following progeny. In the case of $\mathrm{G}_{1} \mathrm{w}^{+}$males that originated from $\mathrm{G} 0$ females and that gave rise only to $\mathrm{w}^{+}$females and to $\mathrm{w}$ females, the insertion could be mapped directly to the $1^{\text {st }}$ chromosome and the $\mathrm{G} 2 \mathrm{w}^{+}$females crossed to the FM7 balancer stock. Independent P-element mediated transgenic lines are listed in Table 5.4 and 5.5.

Tab. 5.4: Drosophila transgenic pJBattP lines generated by single P-element insertions.

\begin{tabular}{lll}
\hline pJBattP line & $\begin{array}{l}\text { locus of insertion } \\
\text { P[w+, tub } \alpha \text {-1-nGFP-attP- }\end{array}$ & viability \\
& SV40] & \\
\hline pJBattPF17.F2 & X chromosome & homozygous viable \\
pJBattPF17.F3 & $3^{\text {rd }}$ chromosome & homozygous viable \\
pJBattPM39 & $3^{\text {rd }}$ chromosome & homozygous lethal \\
pJBattPM40 & $3^{\text {rd }}$ chromosome & homozygous viable \\
pJBattPF15.F2 & $3^{\text {rd }}$ chromosome & homozygous viable \\
pJBattPM2 & $2^{\text {nd }}$ chromosome & homozygous viable \\
pJBattPM44 & $2^{\text {nd }}$ chromosome & homozygous viable \\
pJBattPM15.M1 & $2^{\text {nd }}$ chromosome & homozygous viable \\
\hline
\end{tabular}

Table 5.5: Drosophila transgenic UAS lines generated by single P-element insertions.

\begin{tabular}{|c|c|c|}
\hline Injected construct & UAS line name & $\begin{array}{l}\text { locus of } \\
\text { insertion }\end{array}$ \\
\hline pUASp2-BcdG-6M & UAS-BCDG ${ }^{\text {PMMG }}$ & $1^{\text {st }}$ chromosome \\
\hline pUASp2-BcdG-6M & UAS-BCDG ${ }^{\mathrm{F} 8 \mathrm{M} 1}$ & $1^{\text {st }}$ chromosome \\
\hline pUASp2-BcdD-6M & UAS-BCDD ${ }^{\mathrm{F} 15}$ & $3^{\text {rd }}$ chromosome \\
\hline pUASp2-BcdD-6M & UAS-BCDD ${ }^{\mathrm{M} 29}$ & $3^{\text {rd }}$ chromosome \\
\hline pUASp2-BcdA-6M & UAS-BCDA ${ }^{\mathrm{M} 22}$ & $3^{\text {rd }}$ chromosome \\
\hline pUASp2-BcdA-6M & UAS-BCDA ${ }^{\mathrm{F} 36}$ & $1^{\text {st }}$ chromosome \\
\hline pUASp2-BcdE-6M & UAS-BCDE ${ }^{\mathrm{F} 12}$ & $3^{\text {rd }}$ chromosome \\
\hline pUASp2-BcdE-6M & UAS-BCDE ${ }^{\mathrm{F} 79}$ & $1^{\text {st }}$ chromosome \\
\hline pUASp2-BcdF-6M & UAS-BCDF ${ }^{\mathrm{F9M} 3}$ & $3^{\text {rd }}$ chromosome \\
\hline pUASp2-BcdF-6M & UAS-BCDF ${ }^{\mathrm{M} 22}$ & $3^{\text {rd }}$ chromosome \\
\hline
\end{tabular}




\subsubsection{Drosophila husbandry for expression of UAS-BCD isoforms during early embryogenesis}

Males of UAS-BCD lines (Tab. 5.5) were crossed to virgins of nos-GAL4:VP16 and the progeny collected as embryos, fixed and the cellular localization of the ectopically expressed BCD isoforms identified by $\alpha$-Myc antibody staining.

\subsubsection{Drosophila husbandry for expression of UAS-BCD and sensor constructs during early embryogenesis}

UAS-BCD lines with insertions on the $1^{\text {st }}$ chromosome were crossed into $\mathrm{FM} 7 / \mathrm{w}^{\text {lethal. }} \mathrm{CyO} / \mathrm{Sp}$ background and made homozygous, UAS-BCD lines with insertions on $3^{\text {rd }}$ chromosome were crossed into yw; CyO/if; MKRS/TM6B background and made homozygous. Subsequently the UAS-BCD lines were crossed to sensor lines and made homozygous. These flies were then crossed as males to nos-GAL4:VP16 virgins and the progeny collected as embryos and the GFP expression live imaged. For the two lines of isoform $E$ (UAS-BCDE ${ }^{\mathrm{F} 12}$ and $U A S-B C D E^{F 79}$ ) male flies were crossed to virgins of 38F-Dm3'; nos-GAL4:VP16, the female virgin progeny of this cross collected and crossed to $y w / Y$ males for the collection of embryos. As a control, sensor lines were crossed to virgins of nos-GAL4:VP16. The crosses are summarized in Tab. 5.6. 
General crossing scheme for $1^{\text {st }}$ chromosome UAS-BCD lines, nos-GAL4:VP16 and sensor lines

+ w, UAS-BCD isoform; CyO/Sp $x \partial^{\Uparrow} \mathrm{yw} / \mathrm{Y}$; sensor $\downarrow$ (made homozygous)

o w; nos-GAL4:VP16 x $\partial^{\hat{y}} \mathrm{yw}$, UAS-BCD isoform/Y; sensor

o yw, UAS-BCD isoform/w; sensor/+; nos-GAL4:VP16/+ x $\widehat{\partial} \mathrm{yw} / \mathrm{Y}$

Collection of embryos and live imaging

General crossing scheme for $3^{\text {rd }}$ chromosome UAS-BCD lines, nos-GAL4:VP16 and sensor lines

O yw; CyO/if; UAS-BCD isoform $\mathrm{x} \delta \mathrm{yw} / \mathrm{Y}$; sensor $\downarrow$ (made homozygous)

o w; nos-GAL4:VP16 x $\partial^{\lambda} \mathrm{yw} / \mathrm{Y}$; sensor; UAS-BCD isoform

† yw; sensor/+; UAS-BCD isoform/nos-GAL4:VP16 $\mathrm{x} \overbrace{}^{\Uparrow} \mathrm{yw} / \mathrm{Y}$ $\downarrow$

Collection of embryos and live imaging

General crossing scheme for control cross of sensor line and nos-GAL4:VP16

ㅇ w; nos-GAL4:VP16 x $\overbrace{}^{\Uparrow} \mathrm{yw} / \mathrm{Y}$; sensor

+ yw; sensor/+; nos-GAL4:VP16/+ x $\lesssim$ yw/Y

Collection of embryos and live imaging 
Table 5.6: Crosses for the expression analysis of UAS-BCD and sensor construct during early embryogenesis.

\begin{tabular}{|c|c|c|}
\hline$q$ & & $\pi$ \\
\hline w; nos-GAL4:VP16 & $\mathrm{x}$ & $\mathrm{yw} / \mathrm{Y} ; 38 \mathrm{~F}-\mathrm{Dm} 3$ \\
\hline w; nos-GAL4:VP16 & $x$ & yw, UAS-BCDG ${ }^{\mathrm{F} 4 \mathrm{M} 6} / \mathrm{Y} ; 38 \mathrm{~F}-\mathrm{Dm} 3$ ' \\
\hline w; nos-GAL4:VP16 & $x$ & yw, UAS-BCDG ${ }^{\mathrm{F} 8 \mathrm{M} 1} / \mathrm{Y} ; 38 \mathrm{~F}-\mathrm{Dm} 3$ ' \\
\hline w; nos-GAL4:VP16 & $x$ & yw/Y; 38F-Dm3'; UAS-BCDD ${ }^{\mathrm{F} 15}$ \\
\hline w; nos-GAL4:VP16 & $x$ & yw/Y; 38F-Dm3'; UAS-BCDD ${ }^{\mathrm{M} 29}$ \\
\hline w; nos-GAL4:VP16 & $x$ & yw/Y; 38F-Dm3'; UAS-BCDA ${ }^{\mathrm{M} 22}$ \\
\hline w; nos-GAL4:VP16 & $x$ & $y w$, UAS-BCDA ${ }^{\mathrm{F} 36} / \mathrm{Y} ; 38 \mathrm{~F}-\mathrm{Dm} 33^{\prime}$ \\
\hline w; nos-GAL4:VP16 & $x$ & yw/Y; 38F-Dm3'; UAS-BCDF ${ }^{\text {F9M3 }}$ \\
\hline w; nos-GAL4:VP16 & $x$ & yw/Y; 38F-Dm3'; UAS-BCDF ${ }^{\mathrm{M} 22}$ \\
\hline w; 38F-Dm3'; nos-GAL4:VP16 & $x$ & $\mathrm{yw} / \mathrm{Y}$ \\
\hline w; 38F-Dm3'; nos-GAL4:VP16 & $x$ & $\mathrm{yw} / \mathrm{Y} ; \mathrm{UAS}-\mathrm{BCDE} \mathrm{F}^{\mathrm{F} 12}$ \\
\hline w; 38F-Dm3'; nos-GAL4:VP16 & $x$ & $\mathrm{yw}, \mathrm{UAS}-\mathrm{BCDE}^{\mathrm{F} 79} / \mathrm{Y}$ \\
\hline w; nos-GAL4:VP16 & $x$ & yw/Y; 38F-Dm3'mut \\
\hline w; nos-GAL4:VP16 & $x$ & yw, UAS-BCDG ${ }^{\mathrm{F} 4 \mathrm{M} 6} / \mathrm{Y} ; 38 \mathrm{~F}-\mathrm{Dm} 3$ 'mut \\
\hline w; nos-GAL4:VP16 & $x$ & yw/Y; 38F-Dm3'mut; UAS-BCDD ${ }^{\mathrm{M} 29}$ \\
\hline w; nos-GAL4:VP16 & $x$ & yw/Y; 38F-Hp3' \\
\hline w; nos-GAL4:VP16 & $x$ & yw, UAS-BCDG ${ }^{\mathrm{F} 4 \mathrm{M} 6} / \mathrm{Y} ; 38 \mathrm{~F}-\mathrm{Hp} 3^{\prime}$ \\
\hline w; nos-GAL4:VP16 & $x$ & yw/Y; 38F-Tc3' \\
\hline w; nos-GAL4:VP16 & $x$ & yw, UAS-BCDG ${ }^{\mathrm{F} 4 \mathrm{M} 6} / \mathrm{Y} ; 38 \mathrm{~F}-\mathrm{Tc} 3$ ' \\
\hline w; nos-GAL4:VP16 & $x$ & $\mathrm{yw} / \mathrm{Y} ; 38 \mathrm{~F}-S V 40$ \\
\hline w; nos-GAL4:VP16 & $x$ & $\mathrm{yw}, \mathrm{UAS-BCDG} \mathrm{F}^{\mathrm{FM} 6} / \mathrm{Y} ; 38 \mathrm{~F}-\mathrm{SV} 40$ \\
\hline w; nos-GAL4:VP16 & $x$ & yw/Y; 38F-BRE_257 \\
\hline w; nos-GAL4:VP16 & $x$ & $\mathrm{yw}, \mathrm{UAS}-\mathrm{BCDG}{ }^{\mathrm{F} 4 \mathrm{M} 6} / \mathrm{Y} ; 38 \mathrm{~F}-\mathrm{BRE} \_257$ \\
\hline w; nos-GAL4:VP16 & $x$ & $\mathrm{yw} / \mathrm{Y} ; 38 \mathrm{~F}-\mathrm{H} 1$ \\
\hline w; nos-GAL4:VP16 & $x$ & $\mathrm{yw}, \mathrm{UAS}-\mathrm{BCDG} \mathrm{F}^{\mathrm{F} \mathrm{M} 6} / \mathrm{Y} ; 38 \mathrm{~F}-\mathrm{H} 1$ \\
\hline w; nos-GAL4:VP16 & $x$ & $\mathrm{yw} / \mathrm{Y} ; 38 \mathrm{~F}-\mathrm{H} 2$ \\
\hline w; nos-GAL4:VP16 & $x$ & $\mathrm{yw}, \mathrm{UAS}-\mathrm{BCDG}{ }^{\mathrm{F} 4 \mathrm{M} 6} / \mathrm{Y} ; 38 \mathrm{~F}-\mathrm{H} 2$ \\
\hline
\end{tabular}


Table 5.6 (continued): Crosses for the expression analysis of UAS-BCD and sensor construct during early embryogenesis.

\begin{tabular}{|c|c|c|}
\hline q & & $0^{\lambda}$ \\
\hline w; nos-GAL4:VP16 & $x$ & $\mathrm{yw} / \mathrm{Y} ; 38 \mathrm{~F}-\mathrm{H} 3$ \\
\hline w; nos-GAL4:VP16 & $x$ & yw, UAS-BCDG ${ }^{\mathrm{F} 4 \mathrm{M} 6} / \mathrm{Y} ; 38 \mathrm{~F}-\mathrm{H} 3$ \\
\hline w; nos-GAL4:VP16 & $x$ & yw/Y; 38F-Tc_245 \\
\hline w; nos-GAL4:VP16 & $x$ & $y w$, UAS-BCDG ${ }^{\mathrm{F} 4 \mathrm{M} 6} / \mathrm{Y} ; 38 \mathrm{~F}-\mathrm{Tc} \_245$ \\
\hline
\end{tabular}

\subsubsection{Immunohistochemistry}

\subsubsection{Antibody stainings}

Embryos were dechorionated in $50 \%$ bleach for 1-2 min, washed several times with $\mathrm{dH}_{2} \mathrm{O}$ and PBT. The dechorionated embryos were transferred into glass vials containing equal volumes of fixation solution (1xPEM, 4\% FA) and heptane and fixed by shaking vigorously for $20 \mathrm{~min}$. The fixation solution was removed, $100 \% \mathrm{MeOH}$ added to an equal volume of the heptane and shaken vigorously for $1 \mathrm{~min}$. Devitellinized embryos fell to the bottom of the vial, while the non-devitellinized embryos and embryonic membranes remained in the interphase. The heptane and interphase debris were removed, the embryos washed several times in $100 \% \mathrm{MeOH}$ and immediately rehydrated by washing $1 \mathrm{x}$ in PBT:MeOH [1:1] for 10 min and $4 \times 10$ min washes in PBT. Blocking was performed $1 \mathrm{~h}$ in PBT $+5 \mathrm{x}$ Western blocking (Roche).

Antibody stainings using Rabbit $\alpha-M y c$ as primary antibody were performed at $4^{\circ} \mathrm{C}$ (unless mentioned otherwise) as follows: Rabbit $\alpha$-Myc [dilution 1:100, pre-absorbed] incubated overnight, $3 \times 3$ min washes in PBT, $1 \mathrm{~h}$ blocking in PBT $+0.1 \%$ BSA, incubation of secondary antibody Donkey $\alpha$-Rabbit-AP conjugated antibody [dilution 1:200, pre-absorbed] for $2 \mathrm{~h}, 3 \times 3 \mathrm{~min}$ washes in PBT. All subsequent procedures at RT: $2 \times 3$ min washes in staining buffer, 15 - 20 min incubation in staining solution (1 ml staining buffer $+20 \mu \mathrm{l} \mathrm{NBT/BCIP} \mathrm{Stock} \mathrm{Solution,} \mathrm{Roche),} 3$ rinses in PBT, 10 min wash in PBT, 10 min wash in PBT:EtOH [1:1], 6 x 10 min washes in $100 \% \mathrm{EtOH}, 10 \mathrm{~min}$ 
wash in PBT:EtOH [1:1], $3 \times 10$ min washes in PBT, last wash supplemented with DAPI, mounting in $70 \%$ glycerol and storage at $4^{\circ} \mathrm{C}$.

Antibody stainings using Guinea-pig $\alpha-C A D$ as primary antibody were performed as follows: Guinea-pig $\alpha$-CAD [dilution 1:100, not pre-absorbed] were incubated overnight at $4^{\circ} \mathrm{C}, 3 \times 10$ min washes in PBT, 1 h blocking in PBT $+5 x$ western blocking, incubation of secondary antibody Donkey $\alpha$-Guinea-pig HRP conjugated [dilution 1:500] for $2 \mathrm{~h}$ at RT, 2 rinses in PBT, $3 \times 10$ min washes in PBT, 20 min soaking in 500 $\mu \mathrm{LAB}(0.3 \mathrm{mg} / \mathrm{ml})$ solution $+0.2 \% \mathrm{NiSO}_{4}$, addition of $10-30 \mu \mathrm{l}$ of $0.3 \% \mathrm{H}_{2} \mathrm{O}_{2}$ solution and 20 min color development, 2 rinses in PBT, $3 \times 10$ min washes in PBT, last wash supplemented with DAPI, mounting in $70 \%$ glycerol and storage at $4^{\circ} \mathrm{C}$.

\subsubsection{In situ hybridization}

The ORF of hunchback was liberated from a plasmid (\# 143, M. Averof) and cloned into EcoRI and Xbal sites of pBluescript II KS(+) (pBS-hbORF). The plasmid was linearized with HindIII and $1 \mu \mathrm{g}$ used as template for in vitro transcription with the DIGRNA Labeling Mix and T7 RNA Polymerase (Roche) in a reaction volume of $15 \mu$. The reaction was incubated for $3-4 \mathrm{~h}$ at $37^{\circ} \mathrm{C}, 11 \mu \mathrm{l}$ nuclease-free $\mathrm{H}_{2} \mathrm{O}$ added, $1 \mu \mathrm{l}$ checked on an $1 \% \mathrm{TAE} / \mathrm{EtBr}$ agarose gel for integrity and approximately correct size. Next the reaction was incubated with $25 \mu$ of $2 x$ Carbonate buffer $\left(120 \mathrm{mM} \mathrm{Na}_{2} \mathrm{CO}_{3}\right.$, $80 \mathrm{mM} \mathrm{NaHCO}_{3}, \mathrm{pH} 10.2$ ) for $20 \mathrm{~min}$ at $65^{\circ} \mathrm{C}$ and the following solutions added: $50 \mu \mathrm{l}$ of Stop Solution ( $0.2 \mathrm{M}$ sodium acetate), $10 \mu \mathrm{l} 4 \mathrm{M} \mathrm{LiCl}, 25 \mu \mathrm{l}$ of tRNAs $(4 \mu \mathrm{g} / \mu \mathrm{l})$ and 300 ul $100 \% \mathrm{EtOH}$. The DIG-labeled RNA was precipitated at $-20^{\circ} \mathrm{C}$ for $30 \mathrm{~min}$ or overnight, centrifuged for $30 \mathrm{~min}$ at $4^{\circ} \mathrm{C}$, the pellet was washed with $70 \% \mathrm{EtOH}$, centrifuged for 2 min at RT, air-dried, resuspended on ice in $200 \mu \mathrm{Hyb}$ buffer and stored at $-20^{\circ} \mathrm{C}$.

Embryos were dechorionated in $50 \%$ bleach for $1-2$ min, washed several times with $\mathrm{dH}_{2} \mathrm{O}$ and PBT. The dechorionated embryos were transferred into glass vials containing equal volumes of fixation solution (1xPEM, 4\% FA) and heptane and fixed by shaking vigorously for $25 \mathrm{~min}$. The fixation solution was removed, $100 \% \mathrm{MeOH}$ added to an equal volume of the heptane and shaken vigorously for $1 \mathrm{~min}$. Devitellinized embryos fell to the bottom of the vial, while the non-devitellinized embryos and embryonic membranes remained in the interphase. The heptane and interphase debris were removed and the embryos washed several times in $100 \%$ 
$\mathrm{MeOH}$. The in situ hybridization with the $h b$ probe was performed as follows: 5 min wash in $\mathrm{EtOH}: \mathrm{MeOH}$ [1:1], 3 rinses in $100 \% \mathrm{EtOH}, 1 \mathrm{~h}$ wash in Xylene:EtOH [9:1], 3 rinses in $100 \% \mathrm{EtOH}, 5$ min wash in $100 \% \mathrm{EtOH}, 3$ rinses in $100 \% \mathrm{MeOH}, 5 \mathrm{~min}$ wash in $100 \% \mathrm{MeOH}, 5$ min wash in MeOH:PBT [1:1] + $5 \% \mathrm{FA}$, rinse in PBT + $5 \%$ FA, 25 min post-fixation in PBT $+5 \%$ FA, $4 \times 10$ min wash in PBT, 10 min wash in PBT:Hyb buffer [1:1], 2 min wash in Hyb buffer, 2 -3 h wash in Hyb buffer at $55^{\circ} \mathrm{C}$ with several changes of solution. The $h b$ probe was diluted 1:100 in Hyb buffer, denatured for $5 \mathrm{~min}$ at $80^{\circ} \mathrm{C}$, chilled on ice for $5 \mathrm{~min}$ and pre-heated for $10 \mathrm{~min}$ at $55^{\circ} \mathrm{C}$. The Hyb buffer was aspirated from the embryos, the diluted probe added and incubated for $18 \mathrm{~h}$ at $55^{\circ} \mathrm{C}$. Subsequent steps were: $2 \times 10$ min washes in Hyb buffer at $55^{\circ} \mathrm{C}, 6 \times 30 \mathrm{~min}$ washes in Hyb buffer at $55^{\circ} \mathrm{C}, 10$ min wash in PBT:Hyb buffer [1:1] at RT, $5 \times 10$ min washes in PBT at RT, incubation of pre-absorbed $\alpha$-DIG-AP conjugated antibody (Roche) [dilution 1:2000] overnight at $4^{\circ} \mathrm{C}$. All subsequent procedures at RT: $4 \times 15$ min washes in PBT, $2 \times 3$ min washes in staining buffer, 15 - 20 min incubation in staining solution ( $1 \mathrm{ml}$ staining buffer $+20 \mu \mathrm{l}$ NBT/BCIP Stock Solution, Roche), 3 rinses in PBT, 10 min wash in PBT, 10 min wash in PBT:EtOH [1:1], 6 x 10 min washes in 100 $\% \mathrm{EtOH}, 10 \mathrm{~min}$ wash in PBT:EtOH [1:1], $3 \times 10$ min washes in PBT, last wash supplemented with DAPI, mounting in $70 \%$ glycerol and storage at $4^{\circ} \mathrm{C}$.

\subsubsection{Microscopy and Imaging}

Immunohistochemically stained embryos were analyzed and photographed using Axioskop 2 Plus Zeiss-Biorad Confocal microscope unit.

The analysis of GFP expression in embryos was performed as follows: embryos were collected for $30 \mathrm{~min}$ and aged for $1 \mathrm{~h} 30 \mathrm{~min}$ at $25^{\circ} \mathrm{C}$, dechorionated in $50 \%$ bleach for 1-2 min, washed extensively with tap water, transferred on a microscope slide with a drop of halocarbon oil and covered with a cover glass. Once the halocarbon oil had spread underneath the cover glass the embryos were ready for immediate imaging. The quantitative analysis of fluorescence intensities was performed as follows: Live embryos of similar stage (nuclear division cycle 11) were analyzed and photographed using Leica MZ 16F Stereoscope, Leica DFC 300 FX camera and Leica EL6000 UV lamp source with the green fluorescence filter.

To avoid possible fluctuations in intensity of the UV lamp, the microscope settings and exposure time were kept identical for all experiments within the day. Because 
photobleaching lowered the overall GFP intensity, care was taken to keep exposure to UV light as short as possible during photographing. This was also achieved by deviding the embryos on multiple microscope slides, so that few embryos are being exposed to the UV light at a time. The photographs from each experiment, e.g. embryos of UAS$\mathrm{BCDG}^{\mathrm{F} 4 \mathrm{M} 6} /+; 38 \mathrm{~F}-\mathrm{Dm} 3$ '/+;nos-GAL4:VP16, were always taken against the control embryos (sensor alone: 38F-Dm3'/+;nos-GAL4:VP16/+) and analyzed relative to the control.

For the quantification of fluorescence intensities the same rectangular region of interest (ROI) lying in the center of each embryo was measured using the "Measure" command in ImageJ. Mean values given by the ImageJ program were analyzed using Excel (Microsoft). The average values and relative average values of the fluorescence measurements can be seen in the APPENDIX 6. 


\section{Literature}

Ameres, S., Martinez, J., and Schroeder, R. (2007). Molecular Basis for Target RNA Recognition and Cleavage by Human RISC. Cell 130, 101-112.

Aravin, A.A., Lagos-Quintana, M., Yalcin, A., Zavolan, M., Marks, D., Snyder, B., Gaasterland, T., Meyer, J., and Tuschl, T. (2003). The small RNA profile during Drosophila melanogaster development. Dev Cell 5, 337-350.

Baird-Titus, J.M., Clark-Baldwin, K., Dave, V., Caperelli, C.A., Ma, J., and Rance, M. (2006). The solution structure of the native K50 Bicoid homeodomain bound to the consensus TAATCC DNA-binding site. J Mol Biol 356, 1137-1151.

Bartel, D.P. (2004). MicroRNAs: genomics, biogenesis, mechanism, and function. Cell 116, 281-297.

Bate, M., and Martínez Arias, A. (1993). The Development of Drosophila melanogaster, Volume 1?. 1558.

Bateman, J.R. (2006). Site-Specific Transformation of Drosophila via C31 IntegraseMediated Cassette Exchange. Genetics 173, 769-777.

Becalska, A.N., and Gavis, E.R. (2009). Lighting up mRNA localization in Drosophila oogenesis. Development 136, 2493-2503.

Berleth, T., Burri, M., Thoma, G., Bopp, D., Richstein, S., Friegerio, G., noll, M., and Nüsslein-Vollhard, C. (1988). The role of localization of bicoid RNA in organizing the anterior pattern of the Drosophila embryo. EMBO J 7, 1749-1756.

Betel, D., Wilson, M., Gabow, A., Marks, D.S., and Sander, C. (2008). The microRNA.org resource: targets and expression. Nucleic Acids Research 36, D149-153.

Brennecke, J., Hipfner, D.R., Stark, A., Russell, R.B., and Cohen, S.M. (2003). bantam encodes a developmentally regulated microRNA that controls cell proliferation and regulates the proapoptotic gene hid in Drosophila. Cell 113, 25-36.

Brennecke, J., Stark, A., Russell, R.B., and Cohen, S.M. (2005). Principles of microRNAtarget recognition. Plos Biol 3, e85.

Brown, S.J., Fellers, J.P., Shippy, T.D., Richardson, E.A., Maxwell, M., Stuart, J.J., and Denell, R.E. (2002). Sequence of the Tribolium castaneum homeotic complex: the region corresponding to the Drosophila melanogaster antennapedia complex. Genetics 160, 1067-1074.

Bullock, S.L., Ringel, I., Ish-Horowicz, D., and Lukavsky, P.J. (2010). A'-form RNA helices are required for cytoplasmic mRNA transport in Drosophila. Nat Struct Mol Biol 17, 703709.

Carrington, J.C., and Ambros, V. (2003). Role of microRNAs in plant and animal development. Science 301, 336-338. 
Cassiday, L.A., and MaherllI, L.J. (2002). Having it both ways: transcription factors that bind to DNA and RNA. Nucleic Acids Research 30, 4118-4126.

Chan, S.-K., and Struhl, G. (1997). Sequence-specific RNA binding by Bicoid. Nature 388, 634.

Chawengsaksophak, K., de Graaff, W., Rossant, J., Deschamps, J., and Beck, F. (2004). Cdx2 is essential for axial elongation in mouse development. Proc Natl Acad Sci USA $101,7641-7645$.

Chendrimada, T.P., Finn, K.J., Ji, X., Baillat, D., Gregory, R.I., Liebhaber, S.A., Pasquinelli, A.E., and Shiekhattar, R. (2007). MicroRNA silencing through RISC recruitment of elF6. Nature 447, 823-828.

Cho, P., Gamberi, C., Chopark, Y., Chopark, I., Lasko, P., and Sonenberg, N. (2006). Cap-Dependent Translational Inhibition Establishes Two Opposing Morphogen Gradients in Drosophila Embryos. Current Biology 16, 2035-2041.

Cho, P., Poulin, F., Chopark, Y., Chopark, I., Chicoine, J., Lasko, P., and Sonenberg, N. (2005). A New Paradigm for Translational Control: Inhibition via $5^{\prime}-3^{\prime}$ mRNA Tethering by Bicoid and the elF4E Cognate 4EHP. Cell 121, 411-423.

Copf, T., Rabet, N., Celniker, S.E., and Averof, M. (2003). Posterior patterning genes and the identification of a unique body region in the brine shrimp Artemia franciscana. Development 130, 5915-5927.

Copf, T., Schröder, R., and Averof, M. (2004). From the Cover: Ancestral role of caudal genes in axis elongation and segmentation. Proceedings of the National Academy of Sciences 101, 17711-17715.

Derobertis, E. (2008). Evo-Devo: Variations on Ancestral Themes. Cell 132, 185-195.

Draper, B.W., Mello, C.C., Bowerman, B., Hardin, J., and Priess, J.R. (1996). MEX-3 is a $\mathrm{KH}$ domain protein that regulates blastomere identity in early $\mathrm{C}$. elegans embryos. Cell 87 , 205-216.

Driever, W., and Nüsslein-Volhard, C. (1989). The bicoid protein is a positive regulator of hunchback transcription in the early Drosophila embryo. Nature 337, 138-143.

Dubnau, J., and Struhl, G. (1996). RNA recognition and translational regulation by a homeodomain protein. Nature 379, 694-699.

Dubnau, J., and Struhl, G. (1997). Correction: RNA recognition and translational regulation by a homeodomain protein. Nature 388, 697-697.

Edgar, L.G., Carr, S., Wang, H., and Wood, W.B. (2001). Zygotic expression of the caudal homolog pal-1 is required for posterior patterning in Caenorhabditis elegans embryogenesis. Developmental Biology 229, 71-88.

F. Gilbert, S., and R. Singer, S. (2010). Developmental Biology!: 685. 
Falciani, F., Hausdorf, B., Schröder, R., Akam, M., Tautz, D., Denell, R., and Brown, S. (1996). Class 3 Hox genes in insects and the origin of zen. Proceedings of the National Academy of Sciences 93, 8479-8484.

Ferrandon, D., Koch, I., Westhof, E., and Nüsslein-Volhard, C. (1997). RNA-RNA interaction is required for the formation of specific bicoid mRNA 3' UTR-STAUFEN ribonucleoprotein particles. The EMBO Journal 16, 1751-1758.

Furriols, M., and Casanova, J. (2003). In and out of Torso RTK signalling. EMBO J 22, 1947-1952.

Gamberi, C., Peterson, D.S., He, L., and Gottlieb, E. (2002). An anterior function for the Drosophila posterior determinant Pumilio. Development 129, 2699-2710.

Gregor, T., Tank, D., Wieschaus, E., and Bialek, W. (2007a). Probing the Limits to Positional Information. Cell 130, 153-164.

Gregor, T., Wieschaus, E., Mcgregor, A., Bialek, W., and Tank, D. (2007b). Stability and Nuclear Dynamics of the Bicoid Morphogen Gradient. Cell 130, 141-152.

Grimm, O., Coppey, M., and Wieschaus, E. (2010). Modelling the Bicoid gradient. Development 137, 2253-2264.

Groth, A.C., Fish, M., Nusse, R., and Carlos, M.P. (2003). Construction of Transgenic Drosophila by Using the Site-Specific Intergrase from Phage phiC31. Genetics 166, 17751782.

Hernández, G., Altmann, M., Sierra, J.M., Urlaub, H., Diez del Corral, R., Schwartz, P., and Rivera-Pomar, R. (2005). Functional analysis of seven genes encoding eight translation initiation factor 4E (elF4E) isoforms in Drosophila. Mechanisms of Development 122, 529-543.

Höchsmann, M., Töller, T., Giegerich, R., and Kurtz, S. (2003). Local similarity in RNA secondary structures. Proc IEEE Comput Soc Bioinform Conf 2, 159-168.

Höchsmann, M., Voss, B., and Giegerich, R. (2004). Pure multiple RNA secondary structure alignments: a progressive profile approach. IEEE/ACM Trans Comput Biol Bioinform 1, 53-62.

Horn, C., and Wimmer, E.A. (2000). A versatile vector set for animal transgenesis. Dev Genes Evol 210, 630-637.

Humphreys, D.T., Westman, B.J., Martin, D.I.K., and Preiss, T. (2005). MicroRNAs control translation initiation by inhibiting eukaryotic initiation factor 4E/cap and poly(A) tail function. Proc Natl Acad Sci USA 102, 16961-16966.

Hunter, C.P., and Kenyon, C. (1996). Spatial and Temporal Controls Target pal-1 Blastomere-Specification Activity to a Single Blastomere Lineage in C. elegans Embryos. Cell, 87, 217-226.

Iwasaki, S., Kawamata, T., and Tomari, Y. (2009). Drosophila argonaute1 and argonaute2 employ distinct mechanisms for translational repression. Molecular Cell 34, 58-67. 
Jackson, R.J., and Standart, N. (2007). How do microRNAs regulate gene expression? Sci STKE 2007, re1.

Janody, F., Sturny, R., Schaeffer, V., Azou, Y., and Dostatni, N. (2001). Two distinct domains of Bicoid mediate its transcriptional downregulation by the Torso pathway. Development 128, 2281-2290.

Kiriakidou, M., Tan, G.S., Lamprinaki, S., De Planell-Saguer, M., Nelson, P.T., and Mourelatos, Z. (2007). An mRNA m7G cap binding-like motif within human Ago2 represses translation. Cell 129, 1141-1151.

Lai, E.C., Tomancak, P., Williams, R.W., and Rubin, G.M. (2003). Computational identification of Drosophila microRNA genes. Genome Biol 4, R42.

Leaman, D., Chen, P., Fak, J., Yalcin, A., Pearce, M., Unnerstall, U., Marks, D., Sander, C., Tuschl, T., and Gaul, U. (2005). Antisense-Mediated Depletion Reveals Essential and Specific Functions of MicroRNAs in Development. Cell 121, 1097-1108.

Lemke, S., Busch, S.E., Antonopoulos, D.A., Meyer, F., Domanus, M.H., and Schmidt-Ott, U. (2010). Maternal activation of gap genes in the hover fly Episyrphus. Development 137, $1709-1719$.

Lemke, S., Stauber, M., Shaw, P.J., Rafiqi, A.M., Prell, A., and Schmidt-Ott, U. (2008). Bicoid occurrence and Bicoid-dependent hunchback regulation in lower cyclorrhaphan flies. Evol Dev 10, 413-420.

Li, W.X. (2005). Functions and mechanisms of receptor tyrosine kinase Torso signaling: lessons from Drosophila embryonic terminal development. Dev Dyn 232, 656-672.

Lynch, J.A., Brent, A.E., Leaf, D.S., Pultz, M.A., and Desplan, C. (2006). Localized maternal orthodenticle patterns anterior and posterior in the long germ wasp Nasonia. Nature 439, 728-732.

Lynch, J.A., Peel, A.D., Drechsler, A., Averof, M., and Roth, S. (2010). EGF signaling and the origin of axial polarity among the insects. Curr Biol 20,1042-1047.

Macdonald, P.M., and Struhl, G. (1986). A molecular gradient in early Drosophila embryos and its role in specifying the body pattern. Nature $324,537-545$.

MacDougall, N., Clark, A., MacDougall, E., and Davis, I. (2003). Drosophila gurken (TGFalpha) mRNA localizes as particles that move within the oocyte in two dyneindependent steps. Dev Cell 4, 307-319.

Mathews, D.H., Sabina, J., Zuker, M., and Turner, D.H. (1999). Expanded sequence dependence of thermodynamic parameters improves prediction of RNA secondary structure. J Mol Biol 288, 911-940.

Mathonnet, G., Fabian, M.R., Svitkin, Y.V., Parsyan, A., Huck, L., Murata, T., Biffo, S., Merrick, W.C., Darzynkiewicz, E., Pillai, R.S., et al. (2007). MicroRNA inhibition of translation initiation in vitro by targeting the cap-binding complex elF4F. Science 317 , 1764-1767. 
Mcgregor, A.P. (2005). How to get ahead: the origin, evolution and function ofbicoid. Bioessays 27, 904-913.

Mlodzik, M., Fjose, A., and Gehring, W.J. (1985). Isolation of caudal, a Drosophila homeo box-containing gene with maternal expression, whose transcripts form a concentration gradient at the pre-blastoderm stage. EMBO J 4, No. 11, 2961-2969.

Mlodzik, M., Gibson, G., and Gehring, W.J. (1990). Effects of ectopic expression of caudal during Drosophila development. Development 109, 271-277.

Moor, C., Meijer, H., and Lissenden, S. (2005). Mechanisms of translational control by the 3' UTR in development and differentiation. Seminars in Cell \& Developmental Biology 16, 49-58.

Moreno, E., and Morata, G. (1999). Caudal is the Hox gene that specifies the most posterior Drosophile segment. Nature 400, 873-877.

Müller-Taubenberger, A., Vos, M.J., Böttger, A., Lasi, M., Lai, F.P.L., Fischer, M., and Rottner, K. (2006). Monomeric red fluorescent protein variants used for imaging studies in different species. Eur J Cell Biol 85, 1119-1129.

Murata, Y., and Wharton, R.P. (1995) Binding of pumilio to maternal hunchback mRNA is required for posterior patterning in Drosophila embryos. Cell 80, 747-756.

Niessing, D., Dostatni, N., Jäckle, H., and Rivera-Pomar, R. (1999). Sequence interval within the PEST motif of Bicoid is important for translational repression of caudal mRNA in the anterior region of the Drosophila embryo. EMBO J 18, No. 7, 1966-1973.

Niessing, D., Sprenger, F., Taubert, H., Jäckle, H., and Rivera-Pomar, R. (2000). Homeodomain Position 54 Specifies Transcriptional versus Translational Control by Bicoid. Molecular Cell 5, 395-401.

Olesnicky, E.C., Brent, A.E., Tonnes, L., Walker, M., Pultz, M.A., Leaf, D., and Desplan, C. (2006). A caudal mRNA gradient controls posterior development in the wasp Nasonia. Development 133, 3973-3982.

Pavlopoulos, A., and Averof, M. (2005). Establishing genetic transformation for comparative developmental studies in the crustacean Parhyale hawaiensis. Proc Natl Acad Sci USA 102, 7888-7893.

Peel, A.D. (2008). The evolution of developmental gene networks: lessons from comparative studies on holometabolous insects. Philos Trans R Soc Lond, B, Biol Sci $363,1539-1547$.

Peel, A.D., and Averof, M. (2010). Early asymmetries in maternal transcript distribution associated with a cortical microtubule network and a polar body in the beetle Tribolium castaneum. Dev Dyn 239, 2875-2887.

Peel, A.D., Chipman, A.D., and Akam, M. (2005). Arthropod segmentation: beyond the Drosophila paradigm. Nat Rev Genet 6, 905-916. 
Pillai, R.S., Bhattacharyya, S.N., Artus, C.G., Zoller, T., Cougot, N., Basyuk, E., Bertrand, E., and Filipowicz, W. (2005). Inhibition of translational initiation by Let-7 MicroRNA in human cells. Science 309, 1573-1576.

Pultz, M.A., Diederich, R.J., Cribbs, D.L., and Kaufman, T.C. (1988). The proboscipedia locus of the Antennapedia complex: a molecular and genetic analysis. Genes \& Development 2, 901-920.

Rivera-Pomar, R., Lu, X., Perrimon, N., Taubert, H., and Jäckle, H. (1995). Activation of posterior gap gene expression in the Drosopihla blastoderm. Nature 374, 253-256.

Rivera-Pomar, R., Niessing, D., Schmidt-Ott, U., Gehring, W.J., and Jäckle, H. (1996). RNA binding and translational supression by bicoid. Nature $379,746-749$.

Rørth, P. (1998). Gal4 in the Drosophila female germline. Mechanisms of Development 78, 113-118.

Rosenberg, M.I., Lynch, J.A., and Desplan, C. (2009). Heads and tails: evolution of antero-posterior patterning in insects. Biochim Biophys Acta 1789, 333-342.

Rushlow, C., Doyle, H., Hoey, T., and Levine, M. (1987a). Molecular characterization of the zerknüllt region of the Antennapedia gene complex in Drosophila. Genes \& Development 1, 1268-1279.

Rushlow, C., Frasch, M., Doyle, H., and Levine, M. (1987b). Maternal regulation of zerknült: a homoeobox gene controlling differentiation of dorsal tissues in Drosophila. Nature 330, 583-586.

Sander, K., and Schmidt-Ott, U. (2004). Evo-Devo aspects of classical and molecular data in a historical perspective. J. Exp. Zool. 302B, 69-91.

Savard, J., Tautz, D., Richards, S., Weinstock, G. M., Gibbs, R. A., Werren, J. H., Tettelin, H. \& Lercher, M. J. (2006) Phylogenomic analysis reveals bees and wasps (Hymenoptera) at the base of the radiation of holometabolous insects. Genome Res. 16, 13341338 .

Schaeffer, V., Janody, F., Loss, C., Desplan, C., and Wimmer, E.A. (1999). Bicoid functions without its TATA-binding protein-associated factor interaction domains. Proc Natl Acad Sci USA 96, 4461-4466.

Schoppmeier, M., Fischer, S., Schmitt-Engel, C., Löhr, U., and Klingler, M. (2009). An Ancient Anterior Patterning System Promotes Caudal Repression and Head Formation in Ecdysozoa. Curr Biol 19, 1-5.

Schröder, R. (2003). The genes orthodenticle and hunchback substitute for bicoid in the beetle Tribolium. Nature 422, 621-625.

Schröder, R., Beermann, A., Wittkopp, N., and Lutz, R. (2008). From development to biodiversity--Tribolium castaneum, an insect model organism for short germband development. Development Genes and Evolution 218, 119-126.

Schroeder, R., Barta, A., and Semrad, K. (2004). Strategies for RNA folding and assembly. Nat Rev Mol Cell Biol 5, 908-919. 
Schulz, C., and Tautz, D. (1995). Zygotic caudal regulation by hunchback and its role in abdominal segment formation of the Drosophila Embryo. Development 121, 1023-1028.

Snee, M.J., Arn, E.A., Bullock, S.L., and Macdonald, P.M. (2005). Recognition of the bcd mRNA localization signal in Drosophila embryos and ovaries. Mol Cell Biol 25, 1501-1510.

Sommer, R.J., and Tautz, D. (1991). Segmentation gene expression in the housefly Musca domestica. Development 113, 419-430.

Spirov, A., Fahmy, K., Schneider, M., Frei, E., noll, M., and Baumgartner, S. (2009). Formation of the bicoid morphogen gradient: an mRNA gradient dictates the protein gradient. Development 136, 605-614.

St Johnston, D., and Nüsslein-Volhard, C. (1992). The origin of pattern and polarity in the Drosophila embryo. Cell 68, 201-219.

Stark, A., Brennecke, J., Bushati, N., Russell, R., and Cohen, S. (2005). Animal MicroRNAs Confer Robustness to Gene Expression and Have a Significant Impact on 3' UTR Evolution. Cell 123, 1133-1146.

Stauber, M., Jäckle, H., and Schmidt-Ott, U. (1999). The anterior determinant bicoid of Drosophila is a derived Hox class 3 gene. Proceedings of the National Academy of Sciences 96, 3786-3789.

Stauber, M., Lemke, S., and Schmidt-Ott, U. (2008). Expression and regulation of caudal in the lower cyclorrhaphan fly Megaselia. Dev Genes Evol 218, 81-87.

Stauber, M., Prell, A., and Schmidt-Ott, U. (2002). A single Hox3 gene with composite bicoid and zerknuellt expression characteristics in non-Cyclorrhaphan flies. PNAS 99, 274-279.

Stauber, M., Taubert, H., and Schmidt-Ott, U. (2000). Function of bicoid and hunchback homologs in the basal cyclorrhaphan fly Megaselia (Phoridae). PNAS 97, 10844-1849.

Steinhauer, J., and Kalderon, D. (2006). Microtubule polarity and axis formation in the Drosophila oocyte. Dev Dyn 235, 1455-1468.

Struhl, G., Struhl, K., and Macdonald, P.M. (1989). The gradient morphogen bicoid is a concentration-dependent transcriptional activator. Cell 57, 1259-1273.

Swevers, L., Cherbas, L., Cherbas, P., and latrou, K. (1996). Bombyx EcR (BmEcR) and Bombyx USP (BmCF1) combine to form a functional ecdysone receptor. nsect Biochem. Mol. Biol. 26, 217-221.

Tadros, W., and Lipshitz, H.D. (2005). Setting the stage for development: mRNA translation and stability during oocyte maturation and egg activation in Drosophila. Dev Dyn 232, 593-608.

Thermann, R., and Hentze, M.W. (2007). Drosophila miR2 induces pseudo-polysomes and inhibits translation initiation. Nature 447, 875-878. 
Thorpe, H.M. (1998). In vitro site-specific integration of bacteriophage DNA catalyzed by a recombinase of the resolvase/invertase family. Proceedings of the National Academy of Sciences 95, 5505-5510.

Treisman, J., Gönczy, P., Vashishtha, M., Harris, E., and Desplan, C. (1989). A single amino acid can determine the DNA binding specificity of homeodomain proteins. Cell 59, 553-562.

Tweedie, S., Ashburner, M., Falls, K., Leyland, P., McQuilton, P., Marygold, S., Millburn, G., Osumi-Sutherland, D., Schroeder, A., Seal, R., et al. (2009). FlyBase: enhancing Drosophila Gene Ontology annotations. Nucleic Acids Research 37, D555-D559.

van den Akker, E., Forlani, S., Chawengsaksophak, K., de Graaff, W., Beck, F., Meyer, B.I., and Deschamps, J. (2002). Cdx1 and Cdx2 have overlapping functions in anteroposterior patterning and posterior axis elongation. Development 129, 2181-2193.

van den Berg, A., Mols, J., and Han, J. (2008). RISC-target interaction: cleavage and translational suppression. Biochim Biophys Acta 1779, 668-677.

van Der Zee, M., Berns, N., and Roth, S. (2005). Distinct functions of the Tribolium zerknüllt genes in serosa specification and dorsal closure. Curr Biol 15, 624-636.

Wagner, C., Ehresmann, C., Ehresmann, B., and Brunel, C. (2004). Mechanism of dimerization of bicoid mRNA: initiation and stabilization. J Biol Chem 279, 4560-4569.

Wagner, C., Palacios, I., Jaeger, L., St Johnston, D., Ehresmann, B., Ehresmann, C., and Brunel, C. (2001). Dimerization of the 3'UTR of bicoid mRNA involves a two-step mechanism. J Mol Biol 313, 511-524.

Wimmer, E.A., Carleton, A., Harjes, P., Turner, T., and Desplan, C. (2000). Bicoidindependent formation of thoracic segments in Drosophila. Science 287, 2476-2479.

Wolff, C., Schröder, R., Schulz, C., Tautz, D., and Martin Klingler, M. (1998). Regulation of the Tribolium homologues of caudal and hunchback in Drosophila: evidence for maternal gradient systems in a short germ embryo. Development 125, 2645-2654.

Yeates, D.K., and Wiegmann, B.M. (1999). CONGRUENCE AND CONTROVERSY: Toward a Higher-Level Phylogeny of Diptera. Ann. Rev. Entomol., 34.

Zhao, C., York, A., Yang, F., Forsthoefel, D.J., Dave, V., Fu, D., Zhang, D., Corado, M.S., Small, S., Seeger, M.A., and Ma, J. (2002). The activity of the Drosophila morphogenetic protein Bicoid is inhibited by a domain located outside its homeodomain. Development $129,1669-1680$.

Zuker, M. (2003). Mfold web server for nucleic acid folding and hybridization prediction. Nucleic Acids Res 31, 3406-3415. 
APPENDIX

\section{A1 EMSAs}
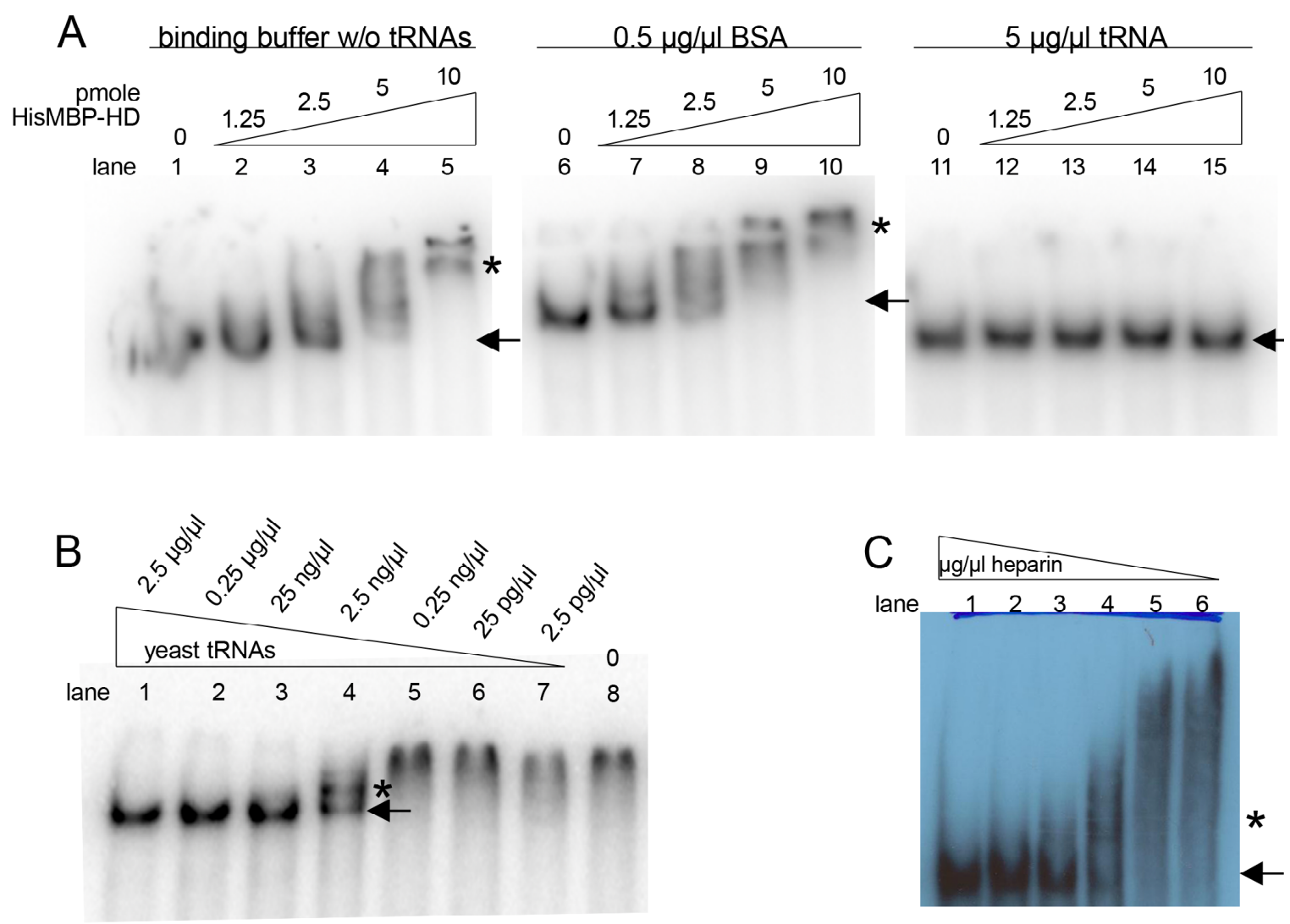

Fig. A1.1: Establishment of BCD homeodomain binding conditions for EMSAs. (A) Effect of $5 \mu \mathrm{g} / \mathrm{\mu l}$ yeast tRNA during RNA-binding of HisMBP-HD. 1.25-10 pmole of HisMBP-HD were incubated with 260 fmole of BRE 164-512 (see Fig. A1.3, B) in the presence of binding buffer without tRNAs (lanes 1-5), with $0.5 \mu \mathrm{g} / \mu \mathrm{l}$ BSA (lanes $6-10$ ) or with $5 \mu \mathrm{g} / \mu \mathrm{l}$ yeast tRNAs (lanes 11-15). In the presences of the $5 \mu \mathrm{g} / \mu \mathrm{l}$ tRNAs the RNA-binding of HisMBP-HD is inhibited (lanes 11-15). The presence of $0.5 \mu \mathrm{g} / \mu \mathrm{l}$ BSA seems to be beneficial to the RNA-binding as a shift occurs at slightly lower HisMBP-HD concentrations than in the absence of BSA (compare lane 3 and 8). (B) Binding of the BRE_164-512 fragment to HisMBP-HD with different yeast tRNA concentrations during RNA binding. Binding of the HisMBP-HD with high tRNA concentrations is inhibited (lanes 1-4), whereas with 2,5 ng/ $\mu$ IRNA of yeast tRNA unspecific binding activity is blocked while binding to the BRE_164-512 can result in a shift (lane 4). tRNA concentrations below $2.5 \mathrm{ng} / \mu \mathrm{l}$ most likely fail to block unspecific binding of HisMBP-HD (lanes 5-8). (C) Effect of different concentrations of heparin (lanes 1-6: $2.25 \mu \mathrm{g} / \mu \mathrm{l} ; 200 \mathrm{ng} / \mu \mathrm{l} ; 18.5 \mathrm{ng} / \mu \mathrm{l} ; 1.6 \mathrm{ng} / \mathrm{\mu l}$; $0.15 \mathrm{ng} / \mathrm{\mu l}$; 0) during RNA-binding of HisMBP-HD. 


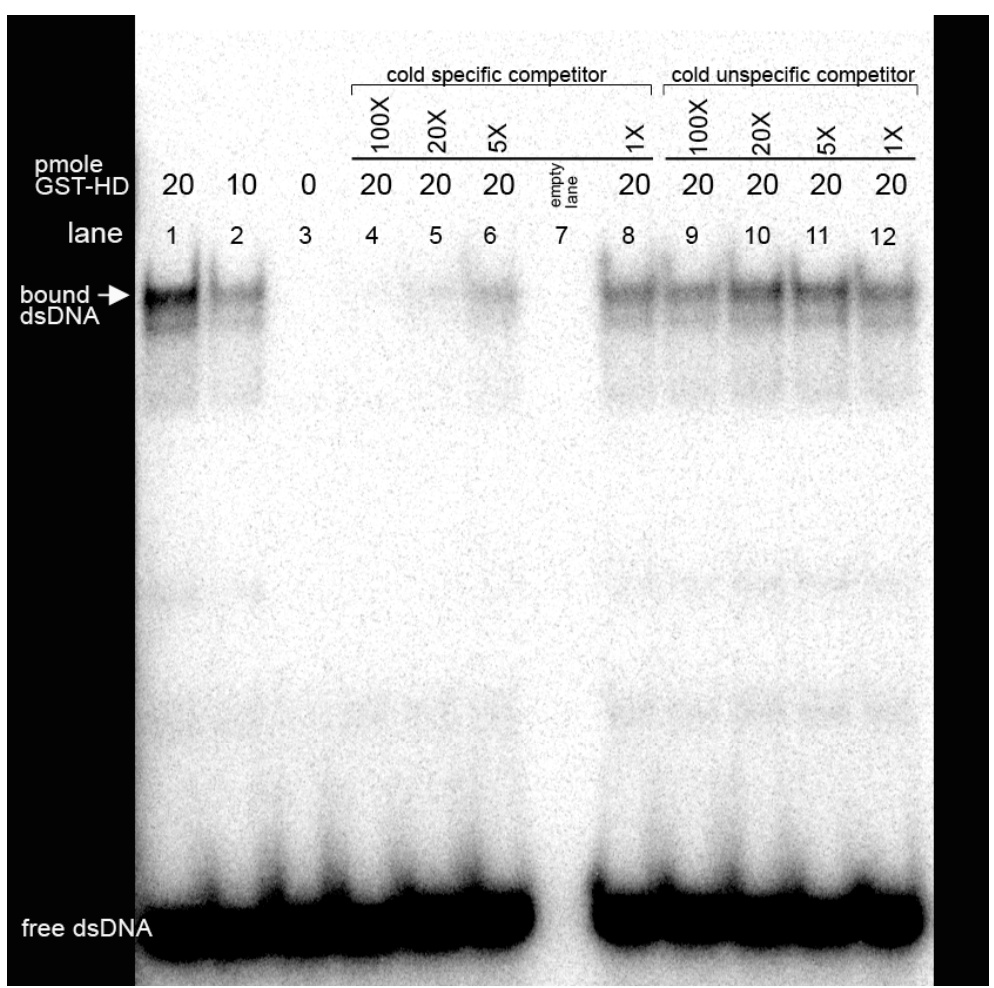

Fig. A1.2: Binding of bacterially expressed GST-HD protein to the BCD ds DNA target fragment (dsDNA) and competition with unlabeld (cold) dsDNA (specific) or cold unspecific DNA (023-F/024-R dsDNA oligo). Lanes 1 and 2 show binding of the dsDNA by the GST-HD (arrow). The dsDNA:GST-HD complex can be competed by an 5-fold to 100 fold excess of unlabeled dsDNA (lanes 4-6), however the unrelated cold unspecific competitor is not able to disrupt the complex (lanes 9-12). 

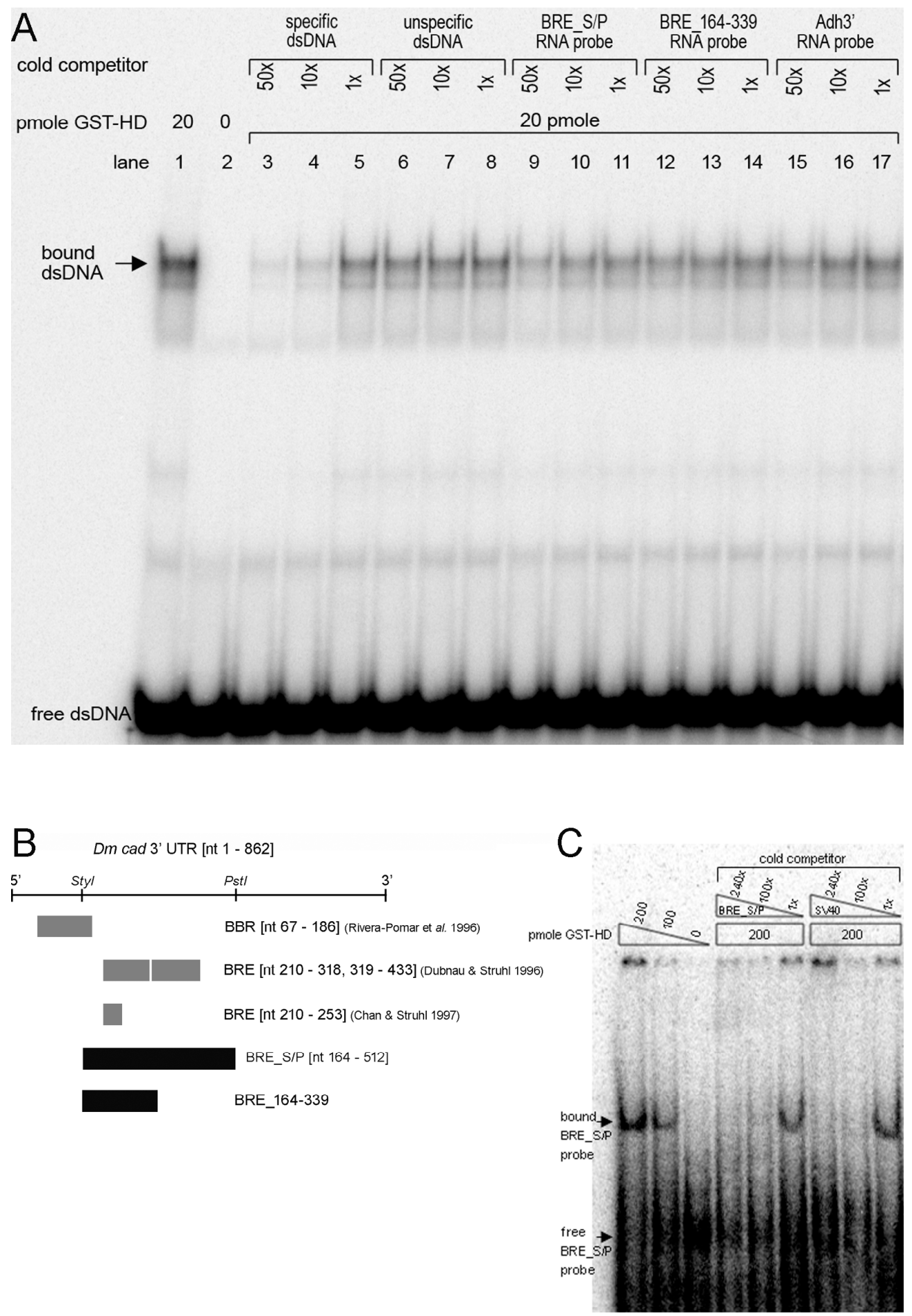

Fig. A1.3: (A) Binding of the GST-HD recombinant protein to the BCD dsDNA target and competition with unlabeled (cold) specific dsDNA (lanes 3-4), unspecific dsDNA (023-F/024-R dsDNA oligo, lanes 6-8), BRE_S/P RNA (lanes 9-11), BRE_164-339 (lanes 12-14) and Adh3' (lanes 15-17). Only a 50-fold and 10-fold excess of the cold specific dsDNA can efficiently compete the labeled dsDNA probe (lanes 3 and 4). In comparison, the RNA probes affect the GST-HD:dsDNA complex to a much lesser degree, with the BRE_S/P probe having the strongest impact, followed by the BRE_164-339 and the Adh3' (compare lanes 9, 12 and 15). (B) Schematic overview of the RNA probes BRE_S/P and BRE_164-339 (black) in comparison to BRE_ fragment of previous studies (grey). (C) Mobility shift assay of the GST-HD recombinant protein bound to the BRE_S/P fragment. Both cold specific (BRE_S/P) and unspecific (SV40) competitors can equally compete the labeled BRE_S/P probe. 

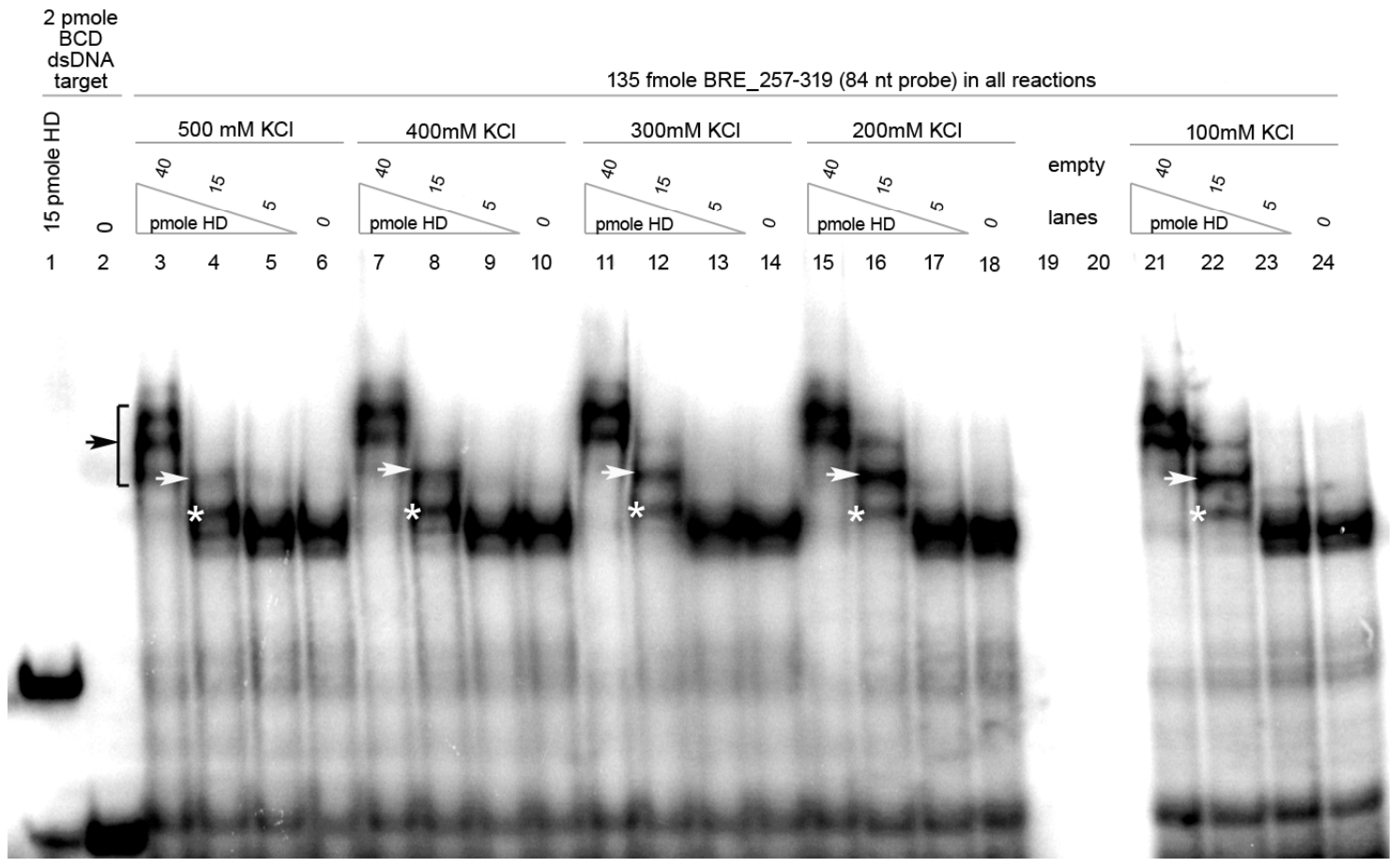

Fig. A.1.4: Binding of the BCD homeodomain to the BRE_257-319 fragment at different salt concentrations (lanes 3-6 $500 \mathrm{mM} \mathrm{KCl}$, lanes 7-10 $400 \mathrm{mM} \mathrm{KCl}$, lanes 11-14 $300 \mathrm{mM}$ $\mathrm{KCl}$, lanes $15-18200 \mathrm{KCl}$, lanes $21-24100 \mathrm{mM} \mathrm{KCl}$ ). Note that the mobility shift of the BRE_257-319 (asterisks) is best visible at 15 pmole homeodomain independently of the salt concentration, however the binding affinity does not increase with elevated salt concentrations. Rather the super-shifts (white arrows) that occur most likely due to unspecific binding of the homeodomain become inhibited at higher salt concentrations. Lanes 1 and 2 show DNA binding as a control for proper functionality of the homeodomain. 


\section{A2 Alignment of cad 3'UTR homologues}

Multalin version 5.4.1

Symbol comparison table: dna

Gap weight: 5

Gap length weight: 0

Consensus levels: high $=90 \%$ low $=50 \%$

$\begin{array}{llllll}\text { MSF : } 1188 & \text { Check: } & & \\ \text { Name: Dmel } & \text { Len: } 1188 & \text { Check: } 129 & \text { Weight: } & 1.00 \\ \text { Name: Dpse } & \text { Len: } 1188 & \text { Check: } 1604 & \text { Weight: } & 1.00 \\ \text { Name: Dwil } & \text { Len: } 1188 & \text { Check: } 7120 & \text { Weight: } & 1.00 \\ \text { Name: Dvir } & \text { Len: } 1188 & \text { Check: } 5257 & \text { Weight: } & 1.00 \\ \text { Name: Dmoj } & \text { Len: } 1188 & \text { Check: } 4085 & \text { Weight: } & 1.00 \\ \text { Name: Consensus } & \text { Len: } 1188 & \text { Check: } 9366 & \text { Weight: } & 0.00\end{array}$

Name: Consensus Len: 1188 Check: 9366 Weight: 0.00

//

1

Dmel UGACACGACC AUU------C CUGUUAUGCG GCGAGCGGCG --.--

Dpse ........UU ..GAGGGGG. .AC.GC.........CU. CCUCUGCUGC CGAUGGACAU CAUCCAAGAG CAGCGACGUC Dwil CC..CA . AU GGUAUCGA UCUAC.-1-

Dvir CAUG.UU.UG CAGGCGCCGU UGAGCC---- - -

Dmoj CCUG.UU.UG GGGCAGGGGA UGAGGU----

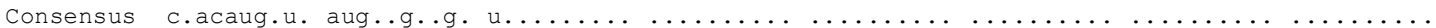

Dmel 81 -- 160

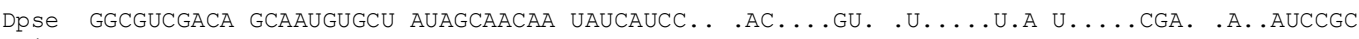

Dwil -- - - - - - - CU AUAACAACAC CAACAACA.U ..C.G....A ...ACA.C.A .A.C. GAAC .AU.A.CA.A

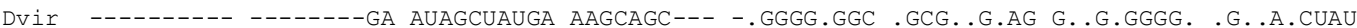

Dmoj -.-.-.-.-.--GC AUGGCUACCA GA--AUG--- -.G.G..G.. .UC.AAU.GA G..G.G.UA .A.AAACAGU

Consensus $\ldots \ldots \ldots \ldots \ldots$........ auagc.ac.a.a.ca.c.a. ag.agc.gcu ..auggagca .c...ag..g .ag.agc...

161

GGGUGGAGUA GGAGGCGUAG GAGGCGUAGG AGGAGGAGGC GGACAACCGU GCGAUGUGUC CUUCAGUGCU

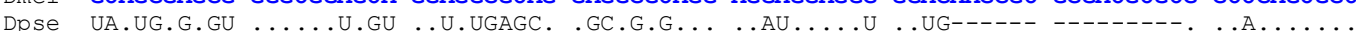

Dwil A.G..A.CA. CA.CUAUA.G .A.CAA.GGC AGCAACA.UC .UCUUUG. .U - . - .

Dvir .G----- CUA..C..CG U.UCCACGU. AUCUG.CC.- U...UUU... -------- ----. UAA GCA.UU..UA

Dmoj UGGAGACCA. CUA..C.ACG U.UUC.AGU. AGCUG.CUU- ..CUUCUA.U - $\ldots$

Consensus $\ldots \ldots \ldots$.g c.gug.ag.g ggu...g.g agc.gg.ag. ag.uu.g.u ............... guacagugcu

241

320

Dmel CCCGCUCUCG CCUUGgACUU GgCUUAACCC UUA-GguCGC GCAgUCAGAC UGUUUCGACC GAACCGAAAA GUUAAUAGGC

Dpse U.U....U. .AG...U. . ............U.. A...U.......GUGA. CCCAA..U. UGGGCG.AUG

Dwil A. AACA.GU …A. UGA. U.GCCGUGGA ..UG. . UAG AGUUGGGACA GUACC. AUU. CC. AAC. GUC AGACUGUCAG

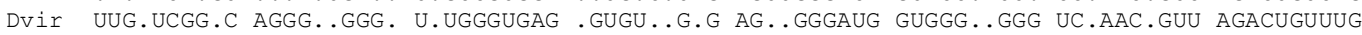

Dmoj .GU.GCAAGC AG..A....G CUUC..CAG. ..GAUAAA.A .A..GAGA.G G.G.GG..GG UGGAGU.GG-

Consensus c.g.c.u.. c.uugga.uu .g..ua...c.u.ggu.g. a.agg.gaa. gg.u.cg..c.caaa.g...g...g..g

321

400

Dmel AgCCGGACGA AUGGAgGACU UGGCGGCCGU UGCACCUGGA AUAUUGCACG UUGUUAA-UU UUUGUGAUUG UAUAUUCCUG

Dpse U.AAU.GGAG .A..UA.UG. G.CGCAG..A $\ldots-$. U. $\ldots \ldots \ldots \ldots \ldots \ldots \ldots \ldots \ldots$

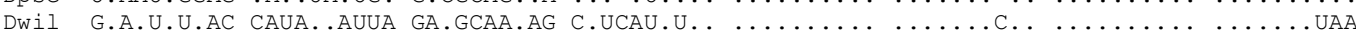

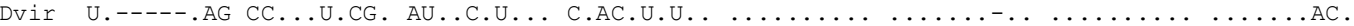

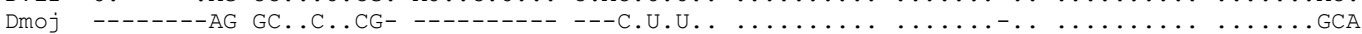

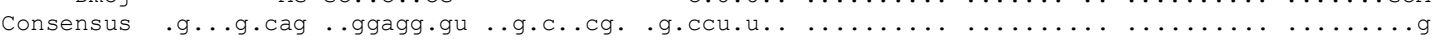

401

Dmel GUUUCGACAC GCGCCAGAgU CCUCACAgCU AAACAAGU--

Dpse ..G.UAUA..... . . CCCAC G......CGC . . ....AAAC CCCUCCAAAA GCUCUCCAAU UUGAUGUAAA UUUGAUAAAC

Dwil .CGCUA.A.- - . . . . AAUA AAAAAGUAGA GGAGUAGAAG AgGUUUUCUG UCUAAGCCAU

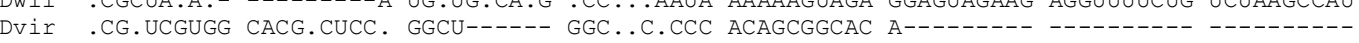

Dmoj .CG.UC.ACG CACG.CUCCA .GC.UUCAAC GGC..C.CCC ACAGCCAGGC AGCAGCAAGA GAAAUUUAUA UUAAUUAAAA

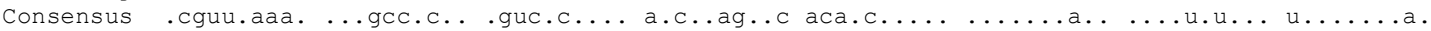

481

C UUAUAUU---

Dmel

Dpse

Dwil

Dvir

Dmoj

UUCUUGUA UUAUGUUUGU UUUUUGUUCA

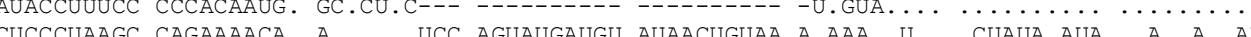

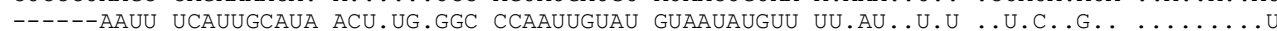

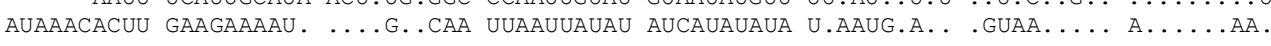

Consensus .u...a.......aa..c .uau.uu.....au..u.u .u.a..... .auauu...a .uuu.u.ugu u.u..g.uca

561

640

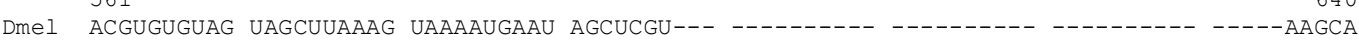

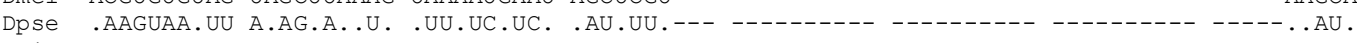

DWil UAU.A. UAUU . UC.ACUUU. ..UGUAC... UAU.GU. UUU UGUUU----- -------- --------A GAACCCUUA.

Dvir .G---.UA.U GUUUAA..U. ...gCGC..G. .UU.GA.UAG UUAGCAUCGA GACACACACA CACACACACA CACGC.CA.

Dmoj CAA.U.U.GU AU.UA.U.U. .UUUG.UUU. UUU.UUGUUC UUAGUUAACA UUUAGCCUAU AAGAAAGACA GAGAC.CAA.

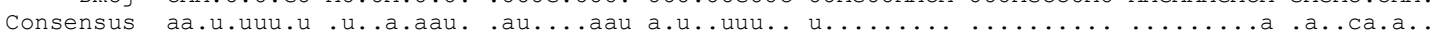

Dmel guAguAAgUA AAgUU--.--

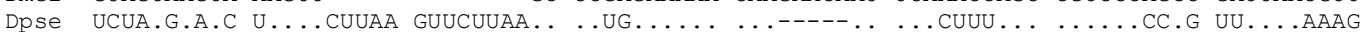

Dwil .A.U.G.A.G U.... UAUAA GUUGCCCA.G 


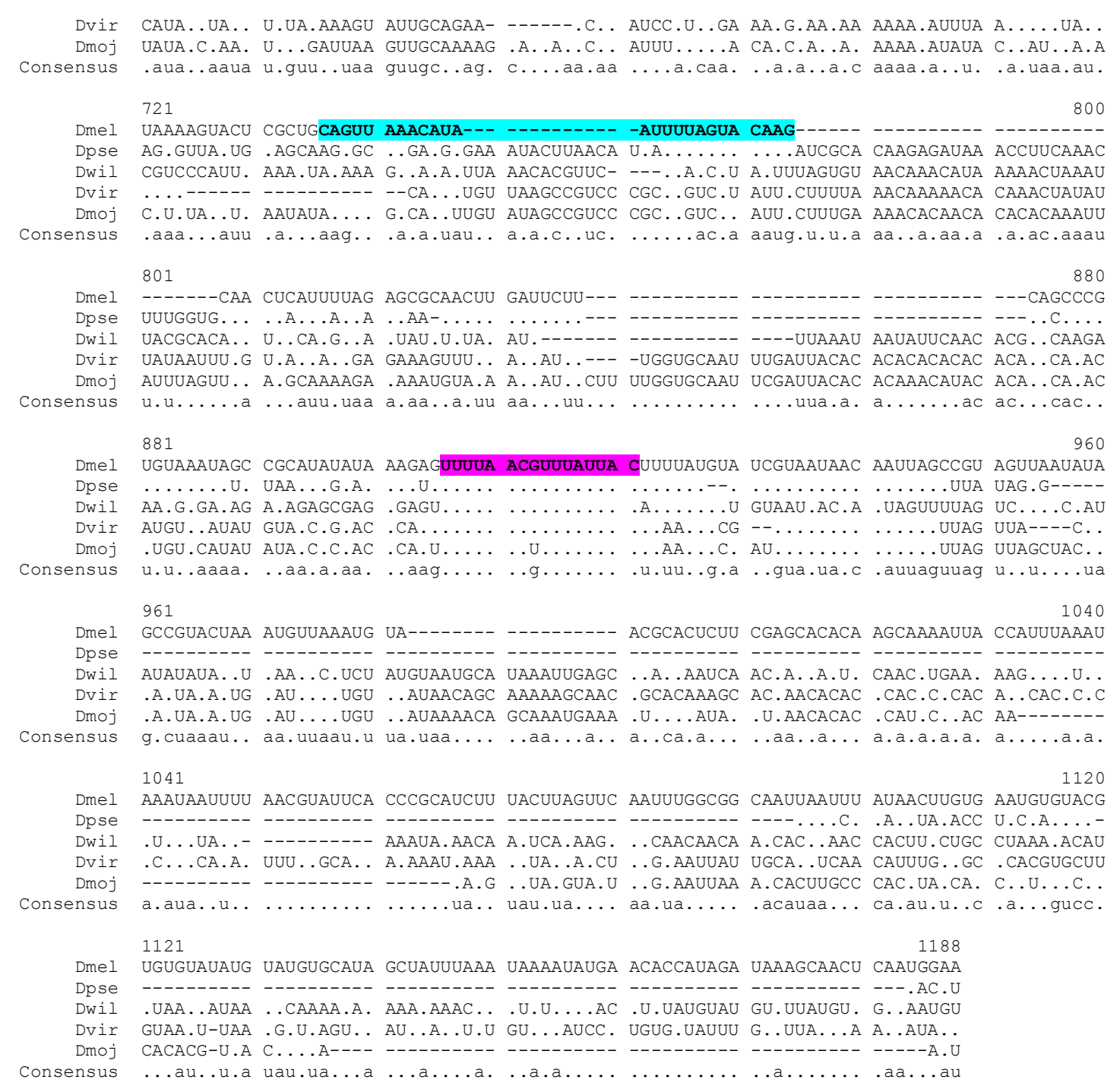

Fig. A2.1: Sequence alignment of cad 3'UTRs from different Drosophila species. The BBR 66-185 (indicated with bold, blue letters) and the BRE_210-253 (indicated with green, bold letter) of the $D$. melanogaster cad 3'UTR do not show extensive sequence similarities to the other Drosophila species. The BRE_257-319 fragment (indicated as yellow box) contains a 38 nt sequence region, which is present in all species (conserved nt are indicated with a point). Interestingly, this box overlaps with a target site of miR-308 (underlined) in Drosophila. The target site of miR-315 is situated further towards the 3' end (indicated with a blue shaded box) (Brennecke et al., 2005; Stark et al., 2005). Another conserved sequence region among these Drosophilid species contains $16 \mathrm{nt}$ spanning nt 906-912 of the D.mel sequence (indicated with a pink shaded box). Alignments were performed with Multalin program version 5.4.1. (Multiple sequence alignment with hierarchical clustering, CORPET, 1988, Nucl. Acids Res., 16 (22), 10881-10890) Dmel $=D$. melanogaster, Dpse $=D$. pseudooscura, Dwil $=D$. willistoni, Dvir $=D$. virillis, Dmoj $=D$. mojavensis. 


\section{A3 Myc-stainings}
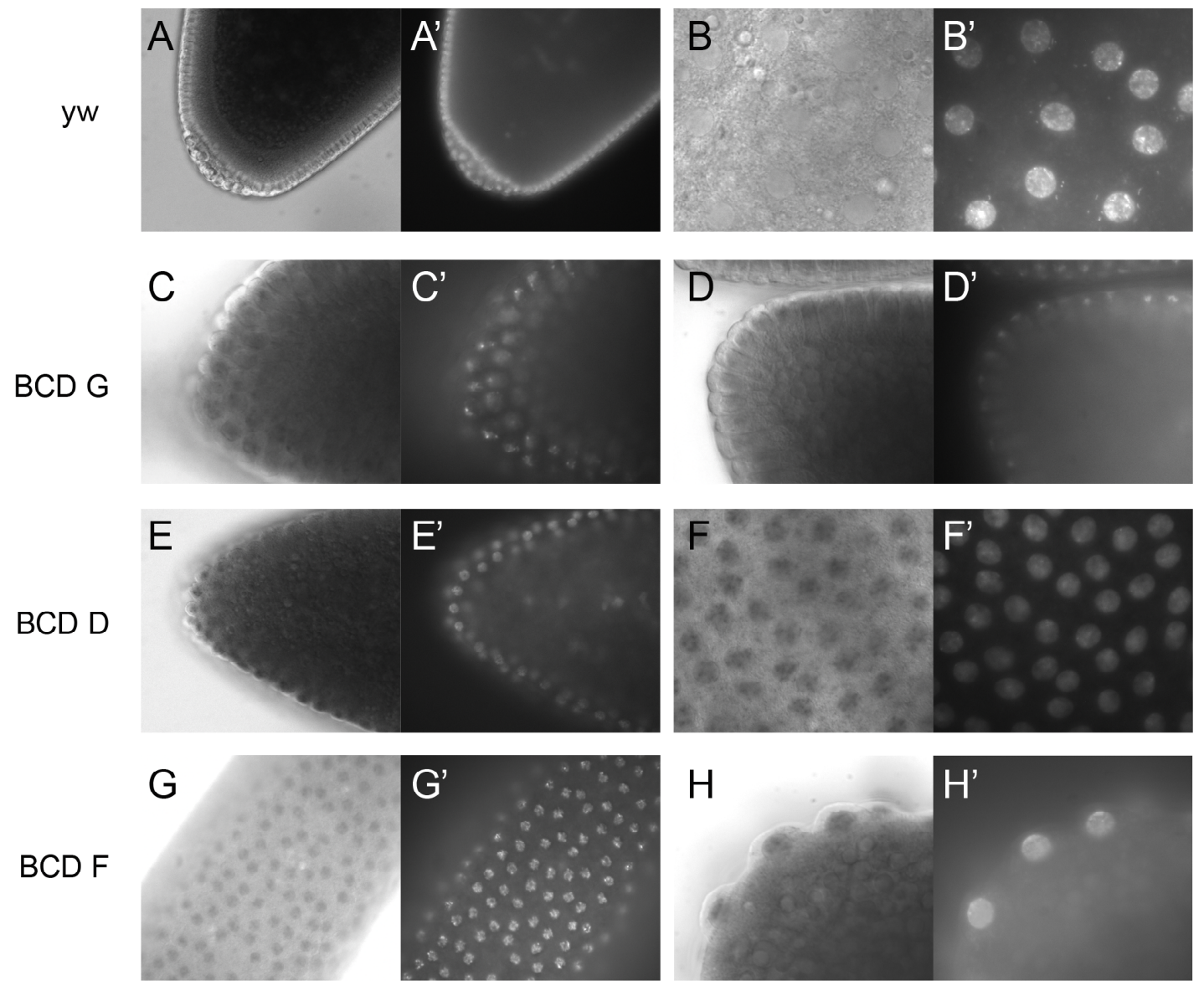

BCD A
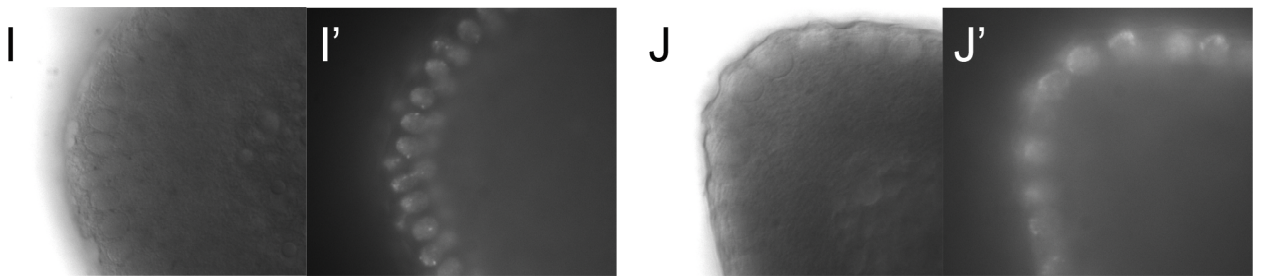

Fig. A3.1: Cellular localization of Myc-tagged BCD isoforms in embryos revealed by $\alpha$ Myc staining. Control embryos (yw) do not show any staining ( $\mathbf{A}$ and $\mathbf{B})$. Embryos derived from females expressing $B C D$ isoform $G$ ( $C$ and $\mathbf{D}$, maternal genotype UAS-BCDG ${ }^{\text {FM6 }} /+$;nosGAL4:VP16/+), isoform D (E and $\mathbf{F}$, maternal genotype UAS-BCDD ${ }^{\mathrm{M} 29} /$ nos-GAL4:VP16) and $^{2}$

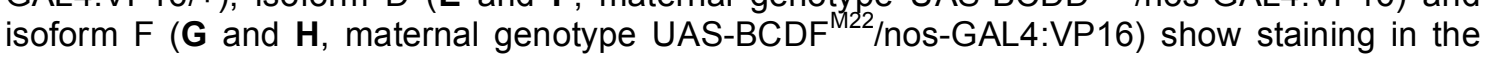
cytoplasm and in the nuclei. Embryos derived from females expressing $B C D$ isoform $A$ show

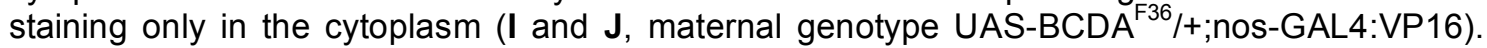
A'-J' show DAPI stainings of the nuclei. All isoforms are expressed ubiquitously, driven by the nos-GAL4:VP16 driver. Because isoform $\mathrm{E}$ was cloned during later stages of $\mathrm{my} \mathrm{PhD}, \alpha-\mathrm{Myc}$ staining could not be performed for isoform $\mathrm{E}$. 


\section{A4 Secondary structure predictions and RNAforester alignments}

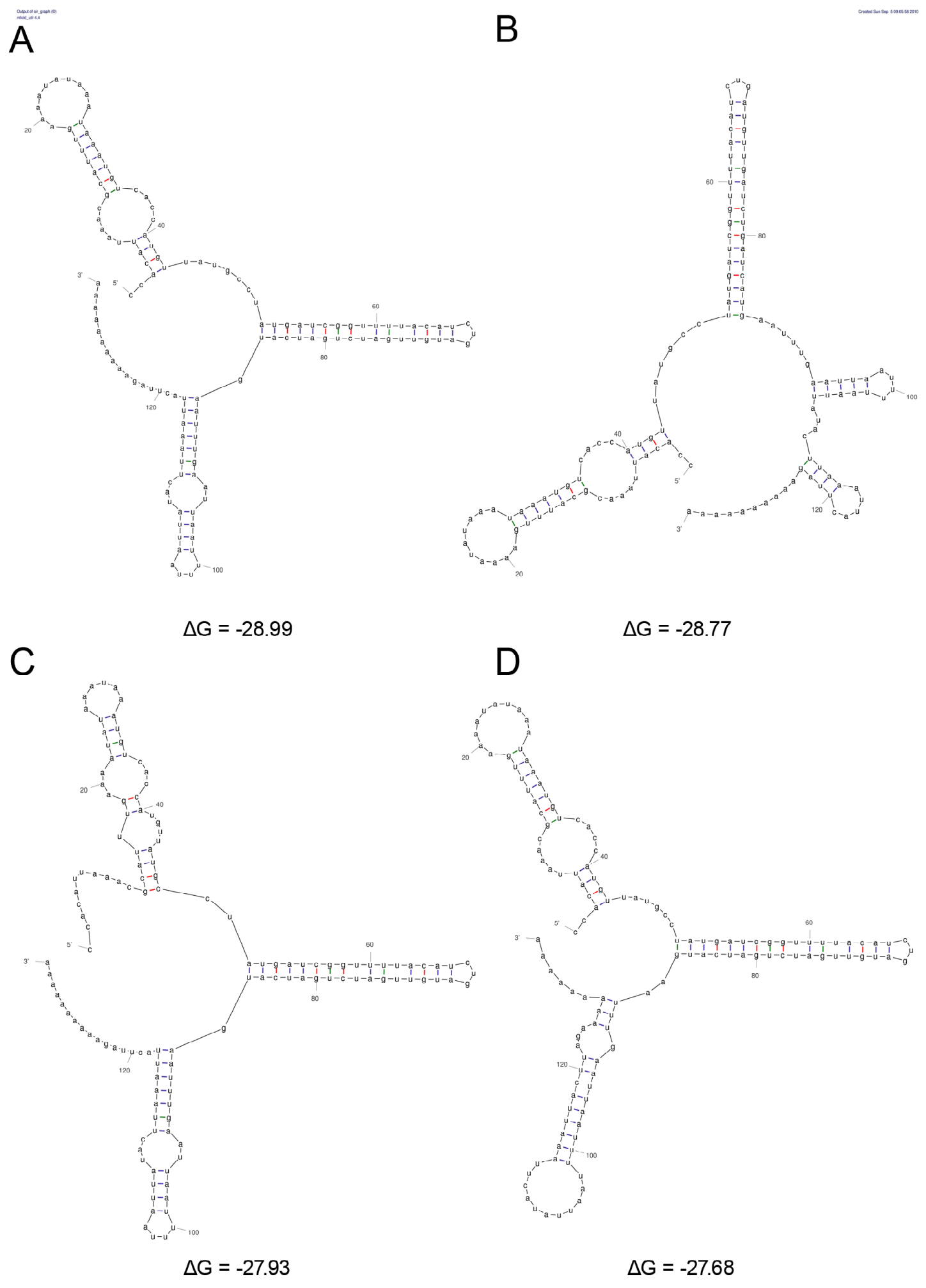

Fig. A4.1 Predicted secondary structures of the Haematopota 3'UTR using the mfold RNA folding program version 2.3. Four secondary structures are predicted, with different $\Delta G$ values (indicated below), which vary mostly in the first and third stem-loop structures. 
BRE_257-319

GGC $\bar{C}$ GUUGCACCUGGAAUAUUGCACGUUGUUAAUUUUUGUGAUUGUAUAUUCCUGGUUUCGA $\ldots((\ldots \ldots(((\cdot(((((((\ldots((((\ldots \ldots \ldots))))) \ldots \ldots))))))) \cdot))) \ldots))$. $(-16.60)$

$\mathrm{H} 1$ GACCACAUUAAACGCAUUUGAAAAUAUAAAUAAAUGUCACCAUGUUAUGCCUCUAGAGC $\ldots . .((((\ldots \ldots)(((((((\ldots \ldots \ldots))))))) \ldots \ldots)))) \ldots((\ldots \ldots))$ $(-6.86)$ GACCACAUUAAACGCAUUUGAAAAUAUAAAUAAAUGUCACCAUGUUAUGCCUCUAGAGC

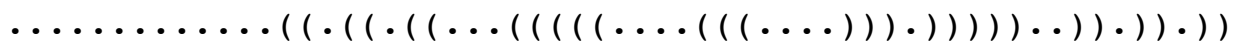
$(-5.87)$

$\mathrm{H} 2$ AUGCCUAUGAUCGGUUUUACAUCUGAUGUUGAUCUGAUCAUGGGCGCGCCA . $(((((((((((((\cdot((\cdot((((\ldots)))) \cdot)) \cdot))))))))))))) \ldots \ldots$ $(-26.99)$

H3 AAUUUGAAUUAAUUUUAAUUAUACUUAAAUUACUUAGGGCGCGCCU $(((((((\ldots((((\ldots)))) \ldots))))))) \ldots(((\ldots \ldots)))$ $(-9.29)$ AAUUUGAAUUAAUUUUAAUUAUACUUAAAUUACUUAGGGCGCGCCU $\ldots \ldots(((((\ldots))))) \ldots(((((\ldots \ldots))))) \ldots \ldots \ldots$ $(-8.97)$

ShSV 40

CUAGAUCAUAAUCAGCCAUACCACAUUUGUAGAGGUUUUACUUGCUUUAAAAAACCUCCCACACCUCCCC $\mathrm{CU}$

$\ldots(((\ldots \ldots))) \ldots \ldots \ldots((\ldots \ldots)) \cdot((((((((\ldots \ldots \ldots \ldots)))))))) \ldots \ldots \ldots$

..

$(-15.83)$

CUAGAUCAUAAUCAGCCAUACCACAUUUGUAGAGGUUUUACUUGCUUUAAAAAACCUCCCACACCUCCCC CU

$\ldots(((\ldots \ldots))) \ldots \ldots \ldots((\ldots \ldots)) \cdot((((((((\ldots(((\ldots))))))))))) \ldots \ldots \ldots$

$\cdots$

$(-15.02)$

Dm3'mut [nt 257-319]

GGCCGUUGCACCUGGAAUAUUGCAGUUUGUUAAUUUUUACGAUUGUAUAUUCCUGGUUUCGA

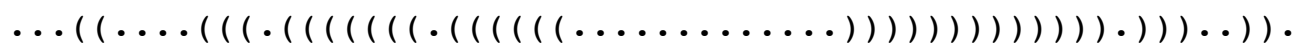
$(-17.36)$

GGCCGUUGCACCUGGAAUAUUGCAGUUUGUUAAUUUUUACGAUUGUAUAUUCCUGGUUUCGA

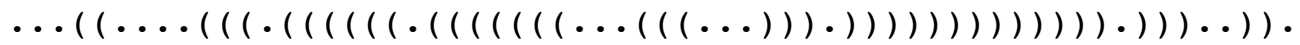
$(-16.95)$

TC'cad 3'UTR only UUCCCUCACAACUCAUAUGACCGUCCCCUUACGUCGAAUGGAAAGAAGACAAUCUUCAGUGUUUGUGAUG AAAUUGUGUUGUGAUUUUGCGUUUUUAUUUAUUUUAUCGAGUCACAUCACCUCGGAGAUUACUGUGCAUA AUUCAUAUAUUUAUCUUUUUGUUACGGCCAUUGUGUAUAUAGUAUUGUAUAAAGGUUUAUCUGUUGUGUA GUAGCAUUUUUAAACUGUUCAUCGGAGUGUGGCACCGGAUUAUCUAGAAAUUCGCACUUUGUAAAUUUGU ACAAACGUUUAUGUGCCUUCUUGUUGCUUCUUCUUCACUAUACCUUGUAAAUAAAGGUCGCUGUAUCCAC GUCUUGUUGUGUUGCAGAGUAGGGGCAGAUUGUAACUUAUUUUUUGGUAUCGAGUGAAGAAUAAAUGAUU UUAUUUGAAAAAAAAAAAAAAAAAAA

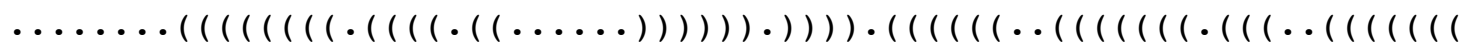
$((((\cdot(((\cdot((((\ldots)))) \ldots \ldots))) \ldots))))) \ldots \ldots)))))) \cdot))) \ldots \ldots))))))((((\cdot(((\ldots$

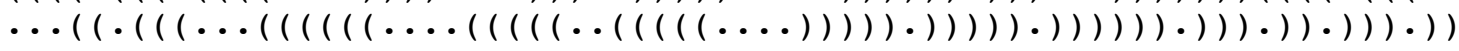




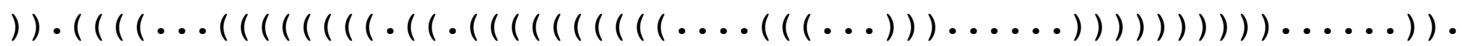

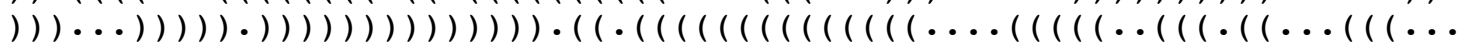
$\left.\left.\left.\left.\left.\left.\left.\left.\left.\left.\left.(((((\cdot((((\ldots)))) \ldots \ldots))))) \cdot))) \cdot)) \cdot)))))))) \ldots()^{\prime}\right)\right)\right) \ldots()\right)\right)\right)\right)\right)\right) \cdot\right)^{\prime}\right) \ldots(($ $((\cdot(((\ldots))) \cdot))) \ldots \ldots \ldots$

$(-125.09)$

TC'cad 5'+ CDS

GUCAAGUGACAAGUGCGUGUGACCCAAAGUGCAAGAACUGAAAGUGUGUGAUGGUCUCGUACUACAACUC GACCAACAUGUACCGUCACCAGCAAGCCGUCGCGGCGCCGGCCAACGCCCCCAUGCACUCAUGGUACGCG GGCUACCAUCAGGGCGCCCAGAUGGGCCCCGAGCAGCAGAUGUGGGAGCCCCAAAUGUGGCACCAUCACA GCCACAUGCCGCCACAUUCGGUCUUUGCGGCCAAUAAUGCCGAGUUCCCGGAGUUCGUGCACUCCGGGAU GGUGCACAACGACGGCACGCAACUGAUGCCGUCGCCCACCGUUUCCGGGAGUGAAAUGUCGAGUCCGGGC GCUGGAAGUGGGAAUUUAUCGCCGCAGAUUCAGACGCAAGUGGCCAGGCCGCCGCCGGCGAGGAGUCCCU AUGAGUGGAUUAAGAAAACCUCCUACCAGAGUCAGCCUAAUCCCGAACCGGCCGAUUUCGCUGACGCUCC GGACGCAAUCGGCAAGACCCGGACGAAAGACAAAUACCGCGUAGUUUACACCGACCACCAGCGAGUGGAG CUCGAAAAAGAGUUCUACUACAGCCGCUACAUCACAAUCCGGCGGAAAGCCGAAUUAGCCAACAGUCUCG GAUUAUCCGAACGUCAAGUGAAAAUCUGGUUCCAGAAUCGUCGUGCUAAGGAACGCAAACAGGUGAAAAA ACGCGAGGAGGUCACCCAGAAGGACAGUCCAAUGAACAUGGGACACCUAACCCAGCAGCAAAUCAUCCAC AACACCCAGACUCAGAUGGGCCAGGCCUAA

$(((\ldots))) \ldots(((\ldots)(((() \ldots \ldots)(((((\ldots))(((\ldots))) \cdot(((\ldots)((((\ldots)) \ldots \ldots)) \ldots$

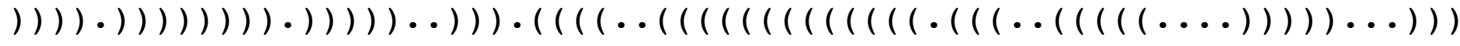
$\cdot(((\ldots \ldots \ldots)(((\cdot((((\ldots \ldots))))))) \cdot))) \cdot((\cdot)(((((((\ldots)((\ldots \ldots)(((((((\ldots \ldots \ldots)$

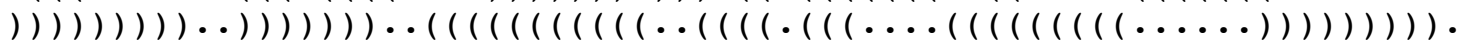

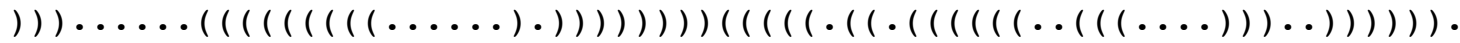

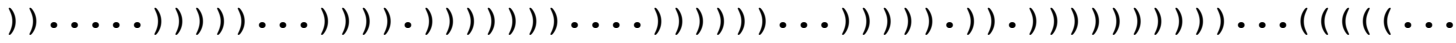

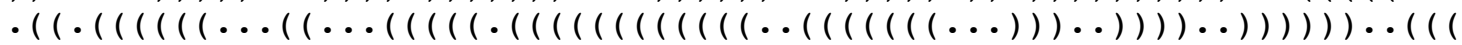

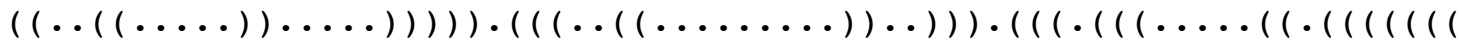

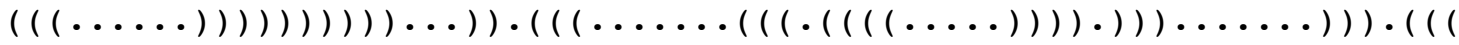
$((\ldots))))(\ldots))) \ldots()) \ldots \ldots()))))(((\cdot((\ldots \ldots)) \ldots \ldots \ldots)) \cdot(((\ldots \ldots \ldots \ldots \ldots$ $\ldots()) \cdot())))((\ldots \ldots)) \cdot(((\ldots))) \ldots((\ldots \ldots(((\ldots \ldots \ldots))) \ldots \ldots(\ldots)))))))$ $\ldots)(\ldots)))) \ldots((((\ldots)))))$. $(-320.34)$

Fig. A4.2 Sequences of BRE_257-319, H1, H2, H3, shSV40, Dm3'mut [nt 257-319], Tc'cad 3'UTR, Tc'cad 5'UTR+coding sequence (CDS) and the predicted secondary structures in Vienna format below. An unpaired base is denoted with a dot, a base pair is denoted with an opening and closing bracket. The opening bracket corresponds to the upstream partner, the closing bracket to the downstream partner of the base base pair. Numbers in parenthesis indicate $\Delta G$ values at $25^{\circ} \mathrm{C}$. For Tc'cad 3'UTR and Tc'cad $5^{\prime} U T R+C D S$ secondary structures with the lowest $\Delta G$ values are shown (for both RNA fragments more than 15 secondary structures were predicted). The region of Tc'cad 5'UTR+CDS that shows highest structural similarity to BRE_257-319 is indicated in grey, the region that shows highest structural similarity to $\mathrm{H} 2$ is indicated in bold letters. Secondary structure predictions were performed using mfold RNA folding program version 2.3 
RNAforester alignments (see below) were performed using the local similarity option. The predicted secondary structures of H1, H2, H3, shSV40, Tc'cad 3'UTR and Tc'cad5'UTR+CDS were aligned against BRE_257-319. The local optimal score indicates the degree of similarity between the two secondary structures as result of the scoring parameters. Highest local optimal scores were calculated for secondary structures of BRE_257-319 and H2 (score $=55$ ) and BRE_257-319 and nt 451-485 of Tc'cad 5"UTR+CDS (numbering begins with the first nt of the cDNA, indicated in grey in Fig. A5.2) (score = 57), which indicates highest structural similarity. In the RNAforester alignment with Tc'cad 5'UTR+CDS and $\mathrm{H} 2$, the same region of the Triboiium RNA is detected to show structural similarity, whereas the similarity score between Tc'cad 5'UTR+CDS and H2 is even higher (score = 95).

RNAforester alignment of BRE_257-319 and H1

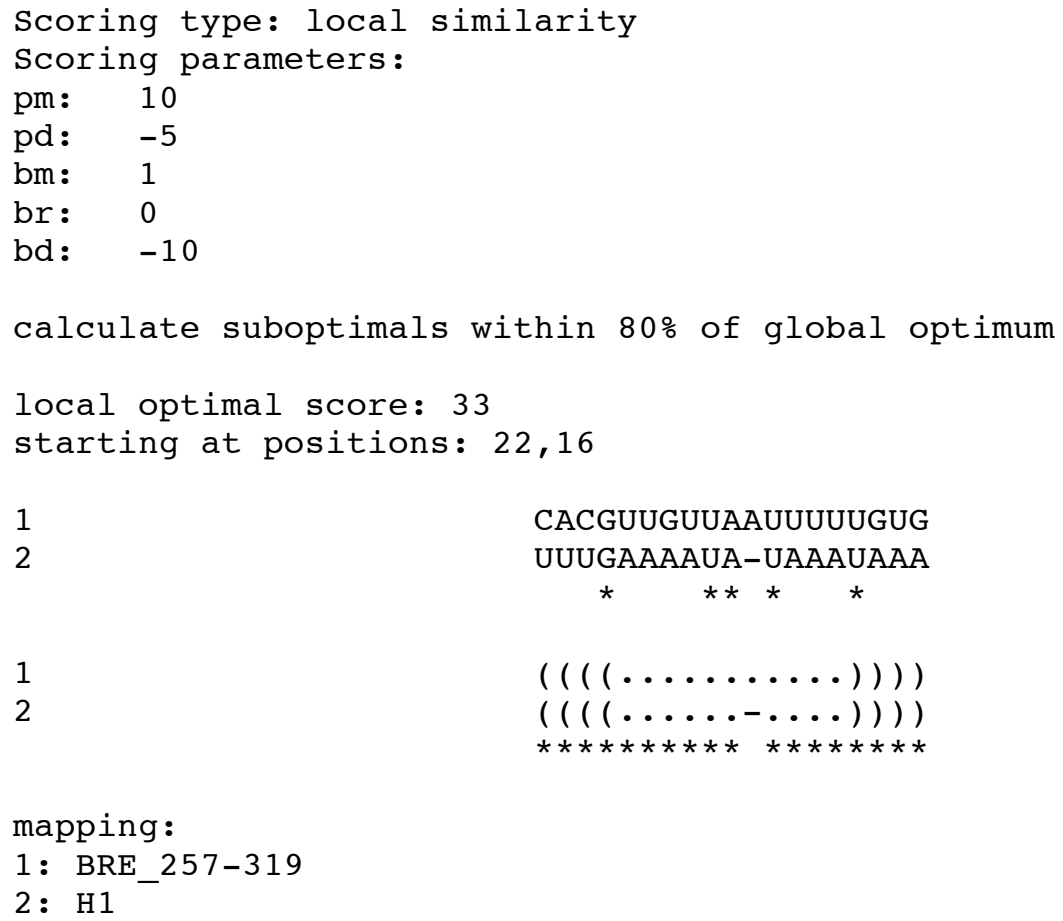


RNAforester alignment of BRE_257-319 and H2

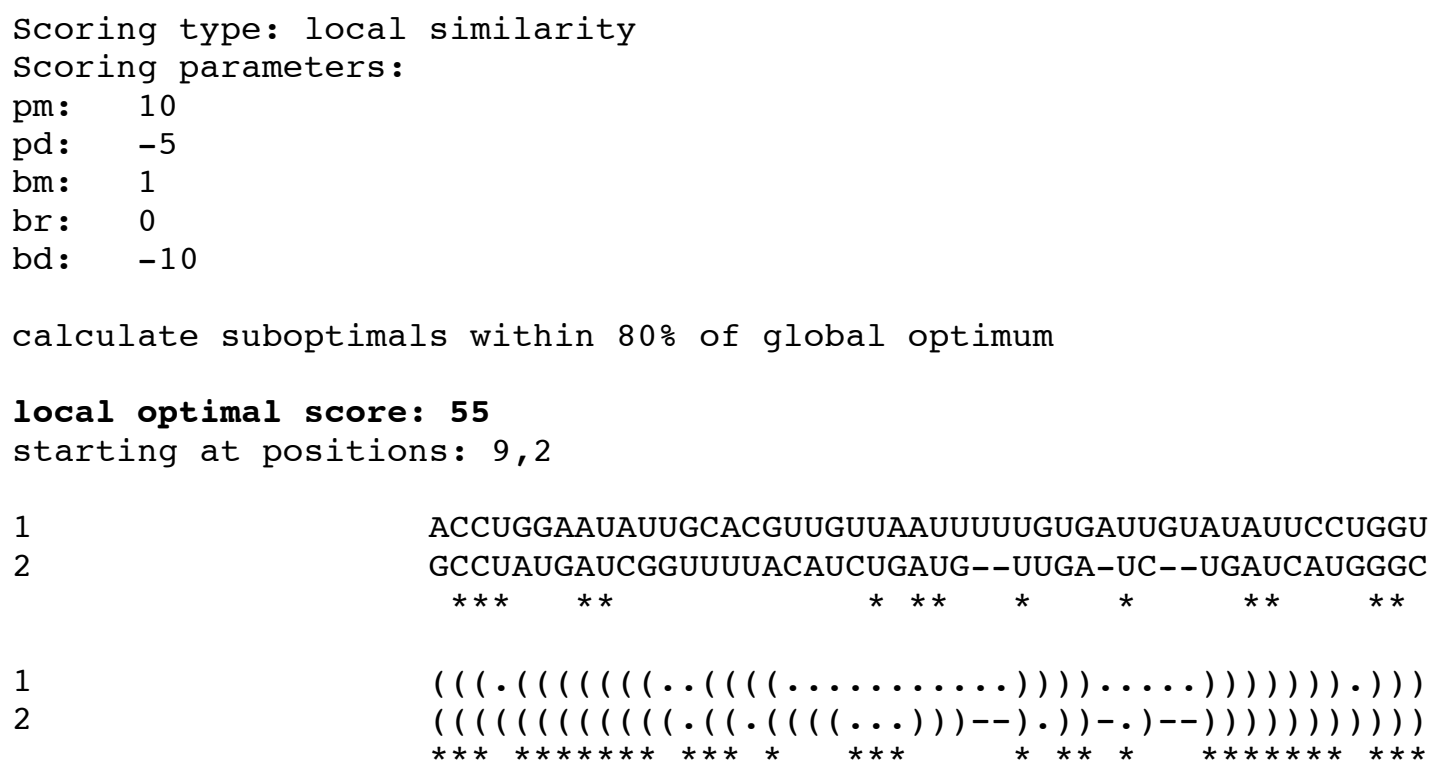

executed command: RNAforester - $-\mathrm{xml}-\mathrm{f}=$ input_data.txt $-2 \mathrm{~d} \quad-1 \quad-\mathrm{so}=80 \quad-\mathrm{bm}=1 \quad-\mathrm{bd}=-10 \quad-\mathrm{br}=0 \quad-\mathrm{pm}=10 \quad-\mathrm{pd}=-5$

RNAforester alignment of BRE_257-319 and H3

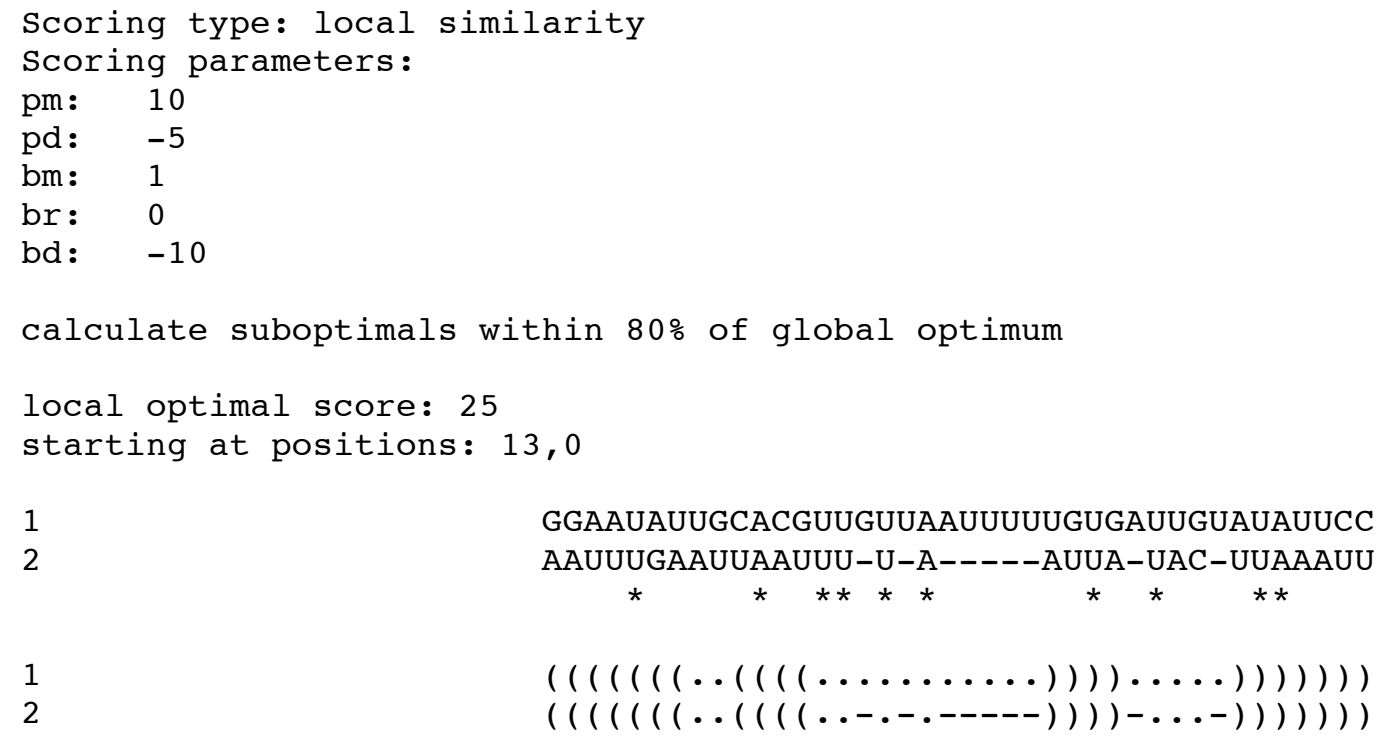




\section{RNAforester alignment of BRE_257-319 and shSV40}

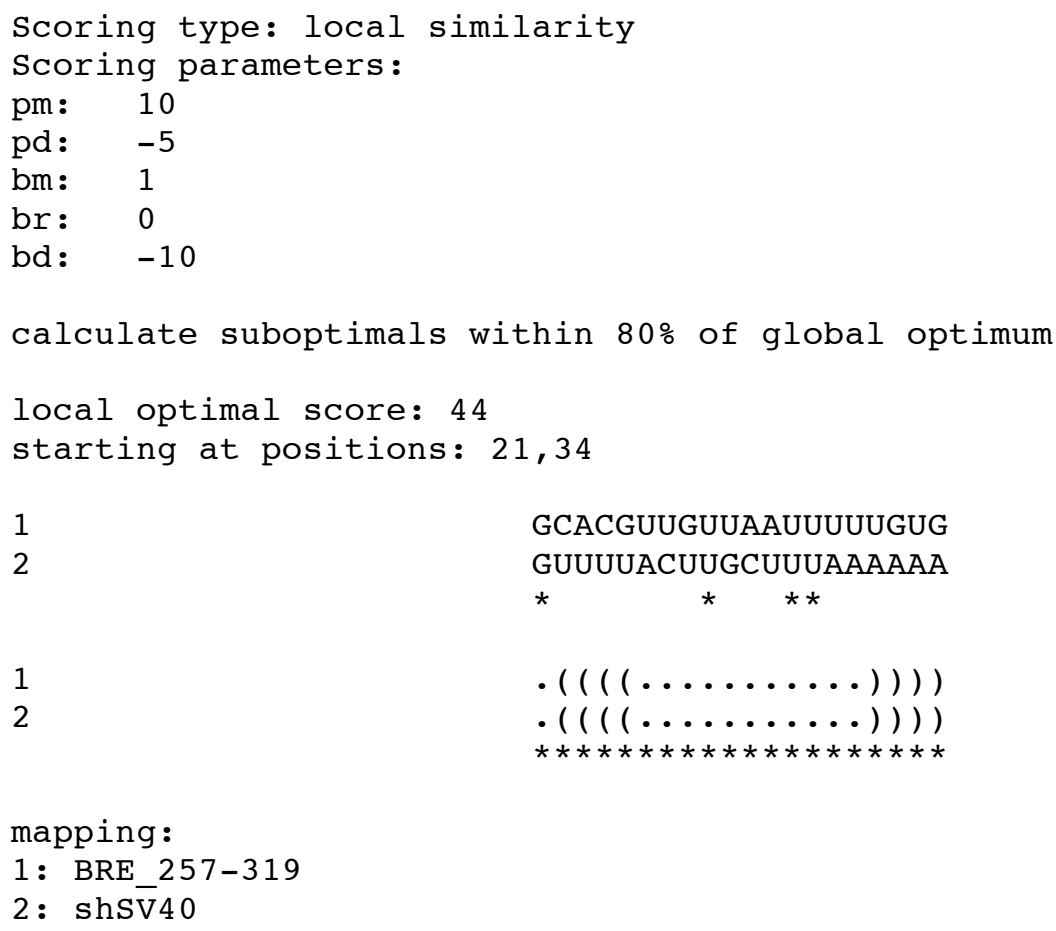

executed command: RNAforester --xml $-\mathrm{f}=$ input_data.txt $-2 \mathrm{~d} \quad-1 \quad-\mathrm{so}=80 \quad-\mathrm{bm}=1 \quad-\mathrm{bd}=-10 \quad-\mathrm{br}=0 \quad-\mathrm{pm}=10 \quad-\mathrm{pd}=-5$

RNAforester alignment of BRE_257-319 and Tc'cad 3'UTR

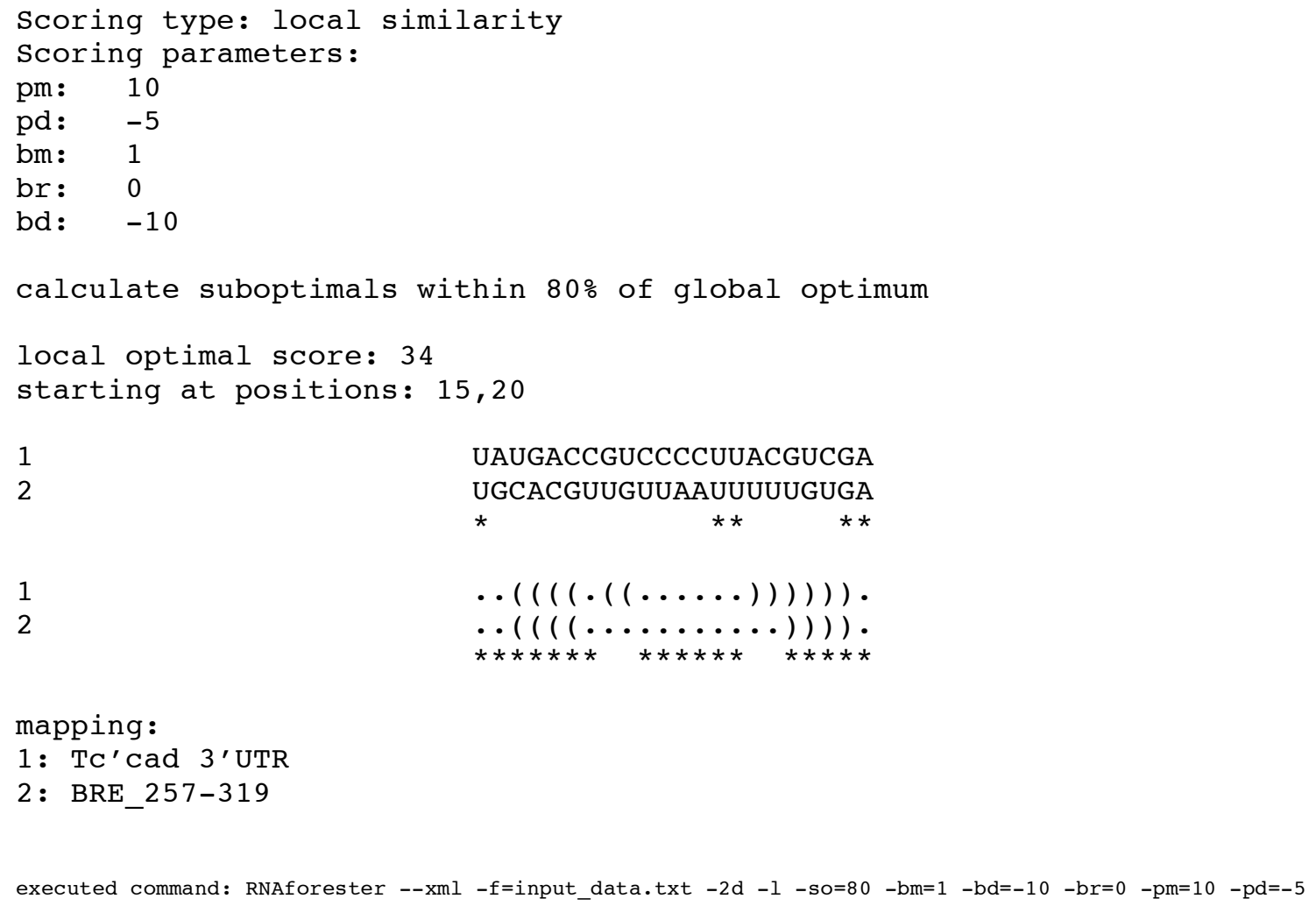


RNAforester alignment of BRE_257-319 and Tc'cad 5'UTR+CDS

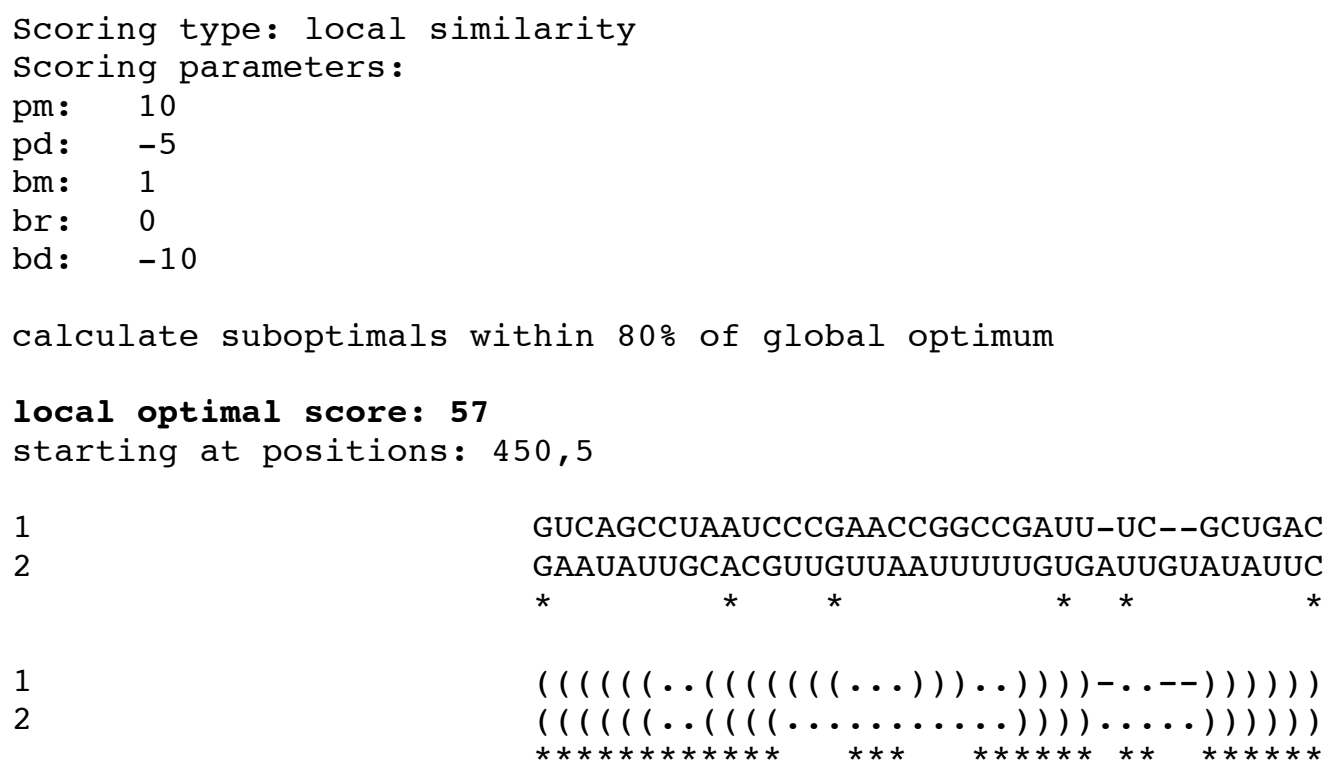

executed command: RNAforester --xml $-\mathrm{f}=$ input_data.txt $-2 \mathrm{~d} \quad-1 \quad-\mathrm{so}=80 \quad-\mathrm{bm}=1 \quad-\mathrm{bd}=-10 \quad-\mathrm{br}=0 \quad-\mathrm{pm}=10 \quad-\mathrm{pd}=-5$

RNAforester alignment of $\mathrm{H} 2$ and Tc'cad 5'UTR+CDS

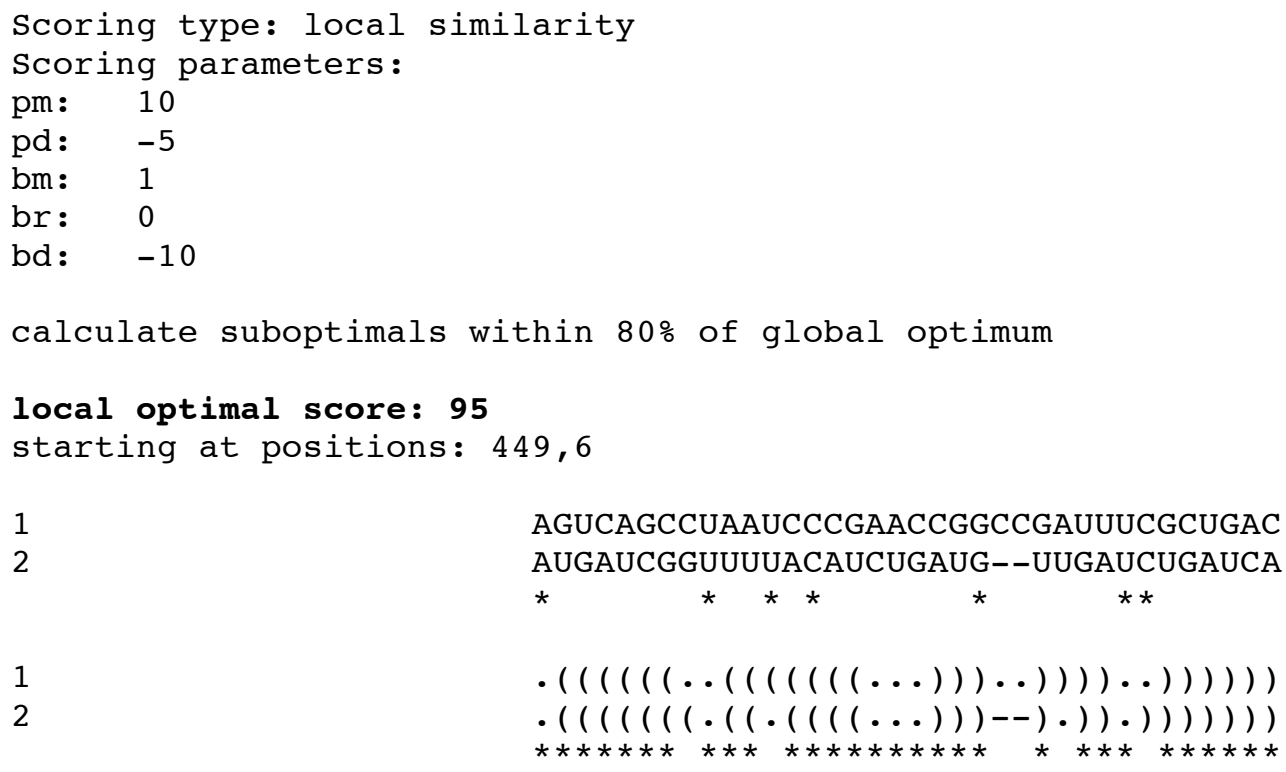




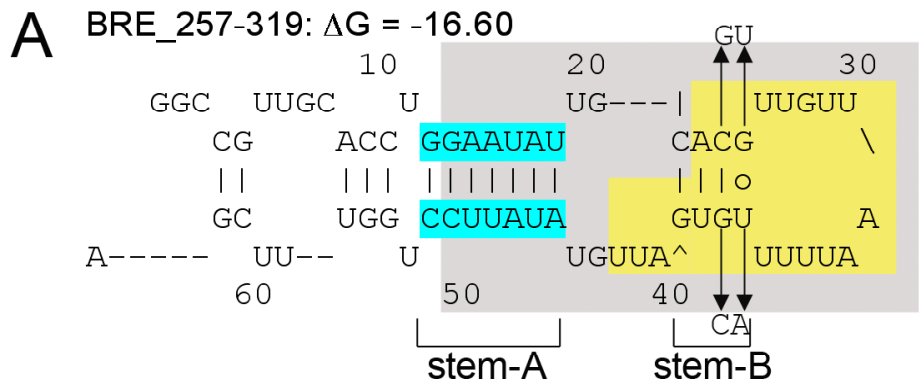

B Dm3'mut [nt 257-319]: $\Delta \mathrm{G}=-17.36$

\begin{tabular}{|c|c|c|c|c|}
\hline & 10 & 2 & & 30 \\
\hline C $\mid \quad U \cup G C$ & c & & & UGUUAA \\
\hline$C G$ & $A C C$ & GGAAUAU & GCAguU & \\
\hline | | & | | | & |||||| $\mid$ & 011010 & \\
\hline $\mathrm{GC}$ & UGG & CCUUAUA & UGUUAG & T \\
\hline$-{ }^{\wedge} \mathrm{UU}--$ & & 50 & 40 & caUUUU \\
\hline
\end{tabular}

Dm3'mut [nt 257-319]: $\Delta \mathrm{G}=-16.95$

\begin{tabular}{cccccc}
\multicolumn{4}{c}{} & \multicolumn{1}{c}{10} & \multicolumn{2}{c}{20} & \multicolumn{2}{c}{30} \\
GGC & UUGC & U & U & UGU & U \\
& CG & ACC & GGAAUA & UGCAguU & UAA
\end{tabular}

Fig. A4.3: Predicted secondary structures of BRE_367-319 and nt 257-319 of Dm3'mut. (A) In the predicted secondary structure of BRE_257-319, the area in grey indicates the sequence that is conserved among different Drosophila species; the area in yellow shows the miR-308 binding target. The changes introduce in 38F-Dm3'mut are indicated by arrows and shown as small bold letters in the predicted secondary structures of Dm3'mut (B). The predicted secondary structures of Dm3'mut (B) and BRE_257-319 (A) are different: stem-B is changed in both structures of Dm3'mut with additional base pairs, however, the mutated nucleotides do not base pair with each other. Stem-A changes in one of the predicted secondary structures, most likely due to the changes of stem-B. Secondary structure predictions were performed using the mfold program (version 2.3). 


\section{A5 Genomic region of bcd}

АTСTCTTCGCTCATCCCTAAATAACGGCACTCTGCAGATGCGAAGCAGTGGATCGCAAAA 1 ACGCAAAATGTGGGCGAAATAAGTTCGCGAGCGTCTCGAAAGTAACCGGTTACTGAAAAT ACAAGAAAGTTTCCACACTCCTTTGCCATTTTTCCGCGCGGCGCTTGGAAATTCGTAAAG

$$
\begin{array}{llllllllllllll}
M & A & Q & P & P & P & D & Q & N & F & Y
\end{array}
$$

ATAACGCGGCGGAGTGTTTGGGGAAAATGGCGCAACCGCCGCCAGATCAAAACTTTTACC $\begin{array}{lllllllllllllllllllllll}\mathrm{H} & \mathrm{H} & \mathrm{P} & \mathrm{L} & \mathrm{P} & \mathrm{H} & \mathrm{T} & \mathrm{H} & \mathrm{T} & \mathrm{H} & \mathrm{P} & \mathrm{H} & \mathrm{P} & \mathrm{H} & \mathrm{S} & \mathrm{H} & \mathrm{P} & \mathrm{H} & \mathrm{P} & \mathrm{H}\end{array}$ ATCATCCGCTGCCCCACACGCACACACATCCGCATCCGCACTCCCATCCGCATCCGCACT

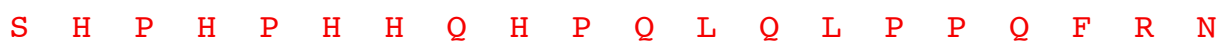
CGCATCCGCACCCACATCACCAACATCCGCAGCTTCAGTTGCCGCCACAATTCCGAAATC $\begin{array}{lllll}P & F & D & L\end{array}$

CСTTCGATTTGgtgagttcccatcgcagcagagaagggctcttgtcccaggaaagctaca gtacagattccctatggtgaacaaacaaccagtgcgatcactgatgaccataaacattta ttgagccgcagcaaatgtgtttctagaacatagggcgaaatcttctattatcttgtttgt gacttttaaagtatcgtagcagaatctaaaaccaattgatattattaatcgttacagtt agtatagtatataattgtatatgaattgtggggcatcatgttattagtgatttgccgaaa tgttctaaaggtgtttcattgaaatggacgaatgttaaacctgttgcactcacaccgac aatcagtaatgtctatttttcaaaagcacatctatggccactgggtatacattattgac tttatacacttcatacaacatattttctaaacaagcattgttgtcctgcatgatgatta gtgaaagtaatattgcaagattcggtccccgaagcgaatcgtcctttcacgtttttatat

$2 \mathrm{a}$

$2 \mathrm{~b}$

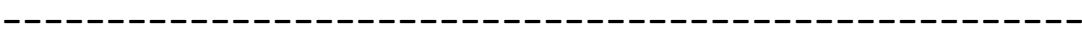

$$
\begin{array}{lllllllllll}
L & F & D & E & R & T & G & A & I & N
\end{array}
$$

aaagacagTGTACCССTTGATTCTTTGAAGCTTTTCGATGAGCGAACGGGAGCGATAAAC

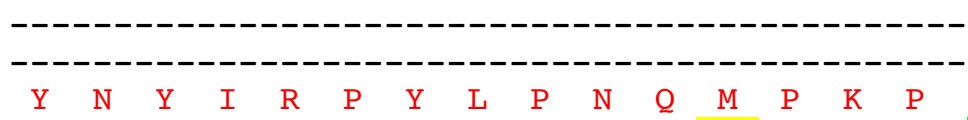

TACAACTACATACGTCCGTATCTGCCCAACCAGATGCCCAAGCCAGgtgagctcaaagce

$3 a$

$3 \mathrm{~b}$

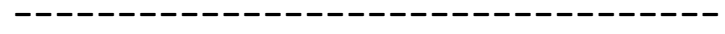

exon

exon

$\begin{array}{lllllllllllll}D & V & F & P & S & E & E & L & P & D & S & L\end{array}$

$\begin{array}{lllllllllllllllllllllllllll}V & M & R & R & P & R & R & T & R & T & T & F & T & S & S & Q & I & A & E & L\end{array}$ GGTGATGCGGCGACCACGTCGCACCCGCACCACTTTTACCAGCTCTCAAATAGCAGAGCT

$\begin{array}{lllllllllllllllllllllllllllllllllll}E & Q & H & F & L & Q & G & R & Y & L & T & A & P & R & L & A & D & L & S & A\end{array}$ GGAGCAGCACTTTCTGCAGGGACGATACCTCACAGCCCCCCGACTTGCGGATCTGTCAGC

$\begin{array}{lllllllllllllllllllll}K & L & A & L & G & T & A & Q & V & K & I & W & F & K & N & R & R & R & R & H\end{array}$ GAAACTAGCCCTGGGCACAGCCCAGGTGAAGATATGGTTTAAGAACCGTCGGCGTCGTCA

$\begin{array}{lllllllllllllllllllll}K & I & Q & S & D & Q & H & K & D & Q & S & Y & E & G & M & P & L & S & P & G\end{array}$ CAAGATCCAATCGGATCAGCACAAGGACCAGTCCTACGAGGGGATGCCTCTCTCGCCGGG

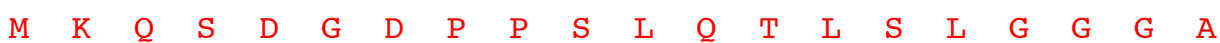
TATGAAACAGAGCGATGGCGATCCCCCCAGCTTGCAGACTCTTAGCTTGGGTGGAGGAGC 
$\begin{array}{llllllllllllllllllll}T & \mathrm{P} & \mathrm{N} & \mathrm{A} & \mathrm{L} & \mathrm{T} & \mathrm{P} & \mathrm{S} & \mathrm{P} & \mathrm{T} & \mathrm{P} & \mathrm{S} & \mathrm{T} & \mathrm{P} & \mathrm{T} & \mathrm{A} & \mathrm{H} & \mathrm{M} & \mathrm{T} & \mathrm{E}\end{array}$ CACGCCCAACGCTTTGACTCCGTCACCCACGCCCTCAACGCCCACTGCACACATGACGGA

$\begin{array}{lllllllllllllllllllll}\mathrm{H} & \mathrm{Y} & \mathrm{S} & \mathrm{E} & \mathrm{S} & \mathrm{F} & \mathrm{N} & \mathrm{A} & \mathrm{Y} & \mathrm{Y} & \mathrm{N} & \mathrm{Y} & \mathrm{N} & \mathrm{G} & \mathrm{G} & \mathrm{H} & \mathrm{N} & \mathrm{H} & \mathrm{A} & \mathrm{Q}\end{array}$ GCACTACAGCGAGTCATTCAACGCСTACTACAACTACAATGGAGGCCACAATCACGCCCA

$\begin{array}{llllllllllllllllllll}A & N & R & H & M & H & M & Q & Y & P & S & G & G & G & P & G & P & G & S & T\end{array}$ GGCCAATCGTCACATGCACATGCAGTATCCTTCCGGAGGGGGGCCAGGACCTGGGTCGAC

$\begin{array}{lllllllllllllllllllllllll}N & V & N & G & G & Q & F & F & Q & Q & Q & Q & V & H & N & H & Q & Q & Q & L\end{array}$ CAATGTCAATGGCGGCCAGTTCTTCCAGCAGCAGCAGGTCCATAATCACCAGCAGCAACT

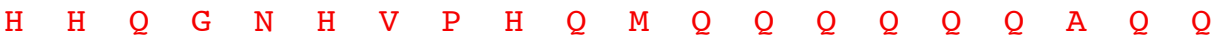
GCACCACCAGGGCAACCACGTGCCGCACCAGATGCAGCAGCAGCAACAGCAGGCTCAGCA

$\begin{array}{llllllllllllllllllll}Q & Q & Y & H & H & F & D & F & Q & Q & K & Q & A & S & A & C & R & V & L & V\end{array}$ GCAGCAATACCATCACTTTGACTTCCAGCAAAAGCAAGCCAGCGCCTGTCGCGTCCTGGT

$\begin{array}{llllllllllllllllllll}K & D & E & P & E & A & D & Y & N & F & N & S & S & Y & Y & M & R & S & G & M\end{array}$ CAAGGACGAACCGGAGGCCGACTACAACTTCAACAGCTCGTACTACATGCGATCGGGAAT

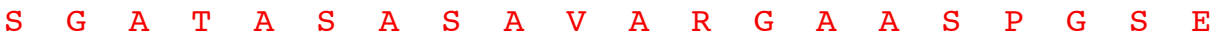
GTCTGGCGCCACTGCATCGGCATCCGCTGTGGCCCGAGGCGCTGCCTCGCCGGGCTCCGA

$\begin{array}{lllllllllllllllllllll}V & Y & E & P & L & T & P & K & N & D & E & S & P & S & L & C & G & I & G & I\end{array}$ GGTCTACGAGCCATTAACACCCAAGAATGACGAAAGTCCGAGTCTGTGTGGCATCGGCAT

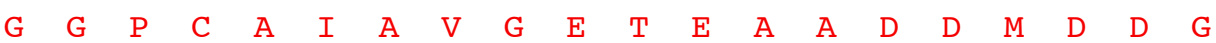
CGGCGGACCTTGCGCCATCGCCGTTGGCGAGACGGAGGCGGCCGACGACATGGACGACGG

$\begin{array}{llllllll}T & \mathrm{~S} & \mathrm{~K} & \mathrm{~K} & \mathrm{~T} & \mathrm{~T} & \mathrm{~L} & \mathrm{Q}\end{array}$

AACGAGCAAGAAGACGACGCTACAGgtcaggcatgagtccacaaccttttttgatctctt gattctgagtgtggcgtttataaattgaagctttaagctttgtaactttcaaactgtctg gtttgagatgttattctgaaagtacttctatttccgatcgatgagatttgggagttctcc aatatttaacatttaacttattaagtttttgttttctaaattagacatggcatttctgaa agggaagtacaagtgttaaagatgtattttaatatagaatttgtatcaaaggttaagatt tcaaccgtttgaaagcccttagttttcagggttttttacttttttattcatgtaatcact cttaatacactgcaagttaaatagcatttctttgaccagaaaataagatctatgcatt ttaaaagtgaaarcagactcatatgctgatgaacatttttagctataaattgtaacaata

atttagcaatttcaatcgaatttatttatgttctaaatgcgttcgctctctccctagATC 4

$\begin{array}{llllllllllllllllllll}L & E & P & L & K & G & L & D & K & S & C & D & D & G & S & S & D & D & M & S\end{array}$ TTGGAGCCTTTGAAGGGTCTGGACAAGAGCTGCGACGATGGCAGTAGCGACGACATGAGC

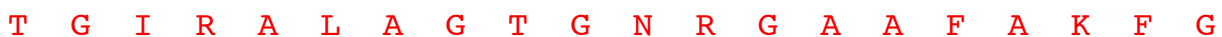
ACCGGAATAAGAGCCTTAGCAGGAACCGGAAATCGTGGAGCGGCATTTGCCAAATTTGGC

$\begin{array}{llllllllllllllllllll}K & P & S & P & P & Q & G & P & Q & P & P & L & G & M & G & G & V & A & M & G\end{array}$ AAGCCTTCGCCCCCACAAGGCCCTCAGCCGCCCCTCGGAATGGGGGGCGTGGCCATGGGC

$\begin{array}{lllllllllllllllllllll}E & S & N & Q & Y & Q & C & T & M & D & T & I & M & Q & A & Y & N & P & H & R\end{array}$ GAATCGAACCAATATCAATGCACGATGGATACGATAATGCAAGCGTATAATCCCCATCGG

$\begin{array}{lllllllllllllll}\mathrm{N} & \mathrm{A} & \mathrm{A} & \mathrm{G} & \mathrm{N} & \mathrm{S} & \mathrm{Q} & \mathrm{F} & \mathrm{A} & \mathrm{Y} & \mathrm{C} & \mathrm{F} & \mathrm{N} & \text { * }\end{array}$

AACGCCGCGGGCAACTCGCAGTTTGCCTACTGCTTCAATTAGCCTGGATGAGAGGCGTGT TAGAGAGTTTCATTAGCTTTAGGTTAACCACTGTTGTTCCTGATTGTACAAATACCAAGT GATTGTAGATATCTACGCGTAGAAAGTTAGGTCTAGTCCTAAGATCCGTGTAAATGGTTC CCAGGGAAGTTTTATGTACTAGCCTAGTCAGCAGGCCGCACGGATTCCAGTGCATATCTT AGTGATACTCCAGTTAACTCTATACTTTCCCTGCAATACGCTATTCGCCTTAGATGTATC TGGGTGGCTGCTCCACTAAAGCCCGGGAATATGCAACCAGTTACATTTGAGGCCATTTGG 
GCTTAAGCGTATTCCATGGAAAGTTATCGTCCCACATTTCGGAAATTATATTCCGAGCCA GCAAGAAAATCTTCTCTGTTACAATTTGACATAGCTAAAAACTGTACTAATCAAAATGAA AAATGTTTCTCTTGGGCGTAATCTCATACAATGATTACCCTTAAAGATCGAACATTTAAA CAATAATATTTGATATGATATTTTCAATTTCTATGCTATGCCAAAGTGTCTGACATAATC AAACATTTGCGCATTCTTTGACCAAGAATAGTCAGCAAATTGTATTTTCAATCAATGCAG ACCATTTGTTTCAGATTCGGAGATTTTTTGCTGCCAAACGGAATAACTATCATAGCTCAC ATTCTATTTACATCACTAAGAAGAGCATTGCAATCTGTTAGGCCTCAAGTTTAATTTTAA AATGCTGCACCTTTGATGTTGTCTCTTTAAGCTTTGTATTTTTAATTACGAAAATATATA AGAACTACTCTACTCGGGTAAATTGTGACTAACTAC

Fig. A5.1: Genomic region of bcd locus 3R:2581564,2585199 (FBgn0000166,) from the Drosophila melanogaster genome (Release 5.31). Sequence was reversed and complemented for illustration in 5' tp 3' orientation. . Untranslated regions are indicated in black and capital letters. The coding sequence is indicated in blue letters, with the translated peptide sequence above in red letters. Intronic sequences are in black and small letters. Splice-donor sites are indicated in green, splice-acceptor sites are indicted in red, alternative splice acceptors are indicated in pink. The start codon of the open reading frame is indicated in light blue. Alternative splicing of exon 2 a results in a premature stop codon (indicated in black and bold letters).

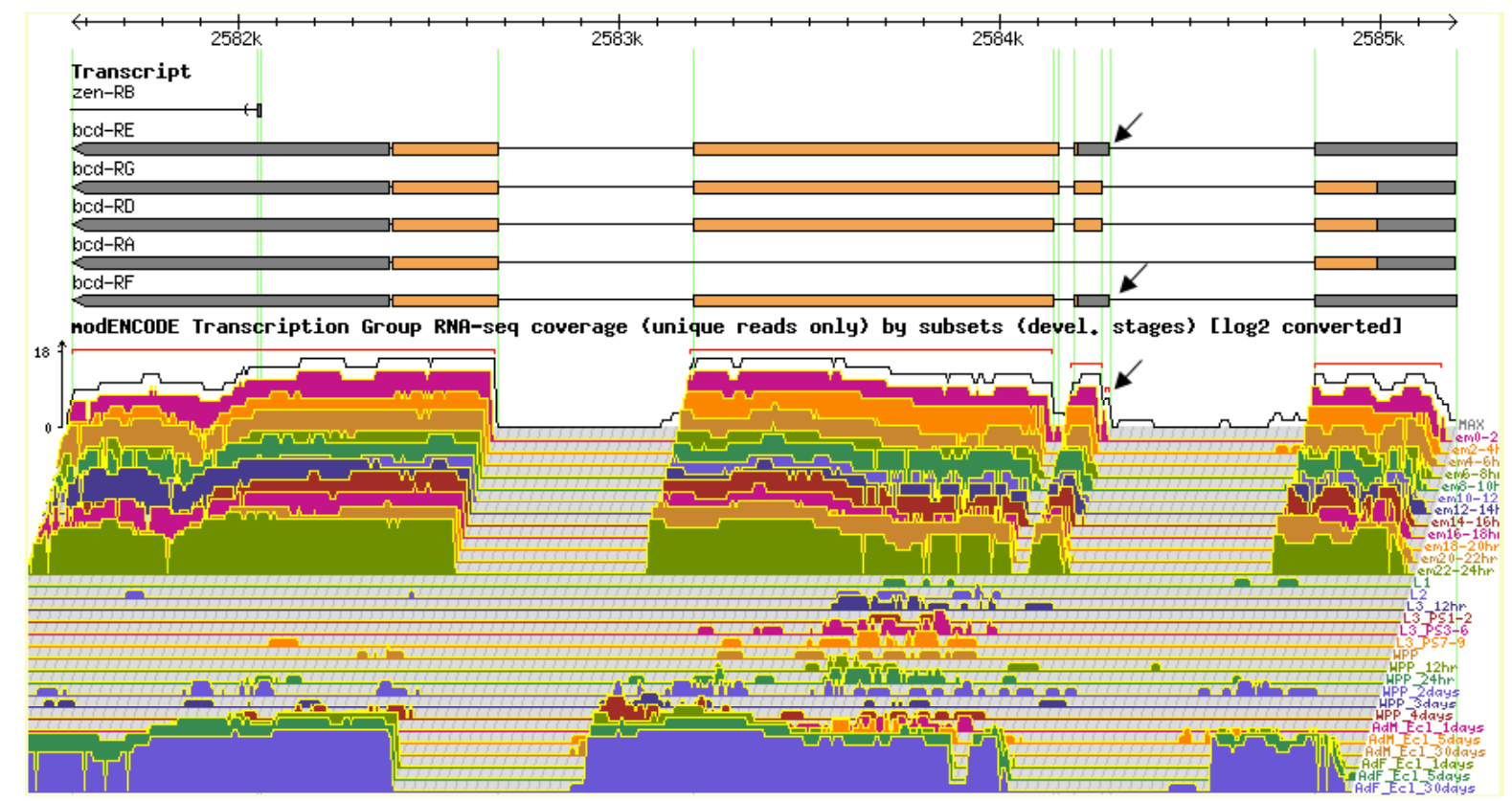

Fig. A5.2: Graphic presentation of RNA sequencing data from FlyBase (Release 5.31). The bcd transcripts are depicted in 3' to 5' orientation, coding sequences are indicated in orange, untranslated regions are indicated in grey. Below, the preview of the data from the Developmental Stage Timecourse Transcriptional profiling with RNA-seq (modENCODE Project). The presence of isoform $E$ and $F$ transcripts were recorded due to their different length (arrows). S. Tweedie, M. Ashburner, K. Falls, P. Leyland, P. McQuilton, S. Marygold, G. Millburn, D. Osumi-Sutherland, A. Schroeder, R. Seal, H. Zhang, and The FlyBase Consortium. FlyBase: enhancing Drosophila Gene Ontology annotations. Nucleic Acids Research (2009) 37: D555-D559; doi:10.1093/nar/gkn788. 


\section{A6 Measurements of in vivo translational reporter assay}

UAS- BCD isoform + 38F-Dm3': Measurements taken from photographs of embryos deriving from females expressing UAS responder line with a BCD isoform, sensor line 38F-Dm3' and nos-GAL4:VP16. Represented in each table are the values of each experiment and the respective control, which was used for normalization (relative average value set to 1 ).

\begin{tabular}{|l|l|l|l|l|l|l|}
\hline maternal genotype & $\mathrm{n}$ & average & se & $\begin{array}{l}\text { Relative } \\
\text { average }\end{array}$ & Relative se & $p$ (ttest) \\
\hline $\begin{array}{l}\text { 38F-Dm3'/+;nos- } \\
\text { GAL4:VP16/+ }\end{array}$ & 22 & 30.904 & 1.702250826 & 1 & 0.055083676 & \\
\hline $\begin{array}{l}\text { UAS-BcdG } \\
\text { Dm3'/+;nos- } \\
\text { GAL4:/38F- }\end{array}$ & 30 & 12.37496667 & 0.44618018 & 0.40044548 & 0.014438086 & $\begin{array}{l}1.86115 \mathrm{E}- \\
10\end{array}$ \\
\hline $\begin{array}{l}\text { UAS-BcdG } \\
\text { Dm3'/+;nos- } \\
\text { GAL4:VP16/+ }\end{array}$ & 23 & 12.9196087 & 0.54752263 & 0.418069724 & 0.017717459 & $\begin{array}{l}2.50359 \mathrm{E}- \\
10\end{array}$ \\
\hline
\end{tabular}

\begin{tabular}{|l|l|l|l|l|l|l|}
\hline maternal genotype & $\mathrm{n}$ & average & se & $\begin{array}{l}\text { Relative } \\
\text { average }\end{array}$ & Relative se & $p$ (ttest) \\
\hline $\begin{array}{l}\text { 38F-Dm3'/+;nos- } \\
\text { GAL4:VP16/+ }\end{array}$ & 31 & 26.78764516 & 0.920271583 & 1 & 0.034354329 & \\
\hline $\begin{array}{l}\text { 38F-Dm3'/+;UAS- } \\
\text { BcdD } \\
\text { GA15/nos- }\end{array}$ & 30 & 7.2609 & 0.356266729 & 0.27105406 & 0.013299666 & $\begin{array}{l}7.29408 \mathrm{E}- \\
22\end{array}$ \\
\hline $\begin{array}{l}\text { 38F-Dm3'/+;UAS- } \\
\text { BcdD } \\
\text { GA29/nos- }\end{array}$ & 35 & 8.450771429 & 0.364582022 & 0.315472725 & 0.013610081 & $\begin{array}{l}5.07777 \mathrm{E}- \\
21\end{array}$ \\
\hline
\end{tabular}

\begin{tabular}{|c|c|c|c|c|c|c|}
\hline maternal genotype & $n$ & average & se & $\begin{array}{l}\text { Relative } \\
\text { average }\end{array}$ & Relative se & $p$ (ttest) \\
\hline $\begin{array}{l}\text { 38F-Dm3'/+;nos- } \\
\text { GAL4VP:16/+ }\end{array}$ & 27 & 41.53422222 & 2.077301275 & 1 & 0.050014209 & 1 \\
\hline $\begin{array}{l}\text { 38-Dm3'/+;UAS- } \\
\text { BcdE }^{\text {F12/nos- }} \\
\text { GAL4:VP16 }\end{array}$ & 24 & 17.23804167 & 0.667302933 & 0.415032249 & 0.01606634 & $\begin{array}{l}2.06896 \mathrm{E}- \\
12\end{array}$ \\
\hline $\begin{array}{l}\text { UAS-BcdE }{ }^{F / 9} /+; 38 \mathrm{~F}- \\
\text { Dm3'/+;nos- } \\
\text { GAL4:VP16/+ }\end{array}$ & 26 & 15.94892308 & 0.60915882 & 0.383994745 & 0.014666431 & $\begin{array}{l}6.67261 \mathrm{E}- \\
13\end{array}$ \\
\hline
\end{tabular}

\begin{tabular}{|l|l|l|l|l|l|l|}
\hline maternal genotype & $\mathrm{n}$ & average & se & $\begin{array}{l}\text { Relative } \\
\text { average }\end{array}$ & Relative se & $p$ (ttest) \\
\hline $\begin{array}{l}\text { 38F-Dm3'/+;nos- } \\
\text { GAL4:VP16/+ }\end{array}$ & 34 & 47.94791176 & 1.081367513 & 1 & 0.022552964 & \\
\hline $\begin{array}{l}\text { 38F-Dm3'/+;UAS- } \\
\text { BcdFF'9M3/nos- } \\
\text { GAL4:VP16 }\end{array}$ & 33 & 20.00727273 & 0.38965492 & 0.417270992 & 0.00812663 & $\begin{array}{l}3.93876 \mathrm{E}- \\
26\end{array}$ \\
\hline $\begin{array}{l}\text { 38F-Dm3'/+;UAS- } \\
\text { BcdF'M22/nos- } \\
\text { GAL4:VP16 }\end{array}$ & 30 & 21.839 & 0.456708889 & 0.455473434 & 0.009525105 & $\begin{array}{l}1.21447 \mathrm{E}- \\
25\end{array}$ \\
\hline
\end{tabular}




\begin{tabular}{|l|l|l|l|l|l|l|}
\hline maternal genotype & $\mathrm{n}$ & average & se & $\begin{array}{l}\text { Relative } \\
\text { average }\end{array}$ & Relative se & $p$ (ttest) \\
\hline $\begin{array}{l}\text { 38F-Dm3'/+;nos- } \\
\text { GAL4:VP16/+ }\end{array}$ & 22 & 25.92740909 & 1.112531548 & 1 & 0.042909476 & \\
\hline $\begin{array}{l}\text { 38F-Dm3'/+;UAS- } \\
\text { BcdA } \\
\text { GAL4:VP16 }\end{array}$ & 29 & 26.83668966 & 0.73565735 & 1.035070244 & 0.028373732 & 0.499543131 \\
\hline $\begin{array}{l}\text { UAS-BcdA } \\
\text { Dm36/++;/nos- } \\
\text { GAL4:VP16/+ }\end{array}$ & 32 & 26.3239375 & 0.662281522 & 1.015293792 & 0.025543683 & 0.761194307 \\
\hline
\end{tabular}

UAS BCD isoforms + sensor: Measurements taken from photographs of embryos deriving from females expressing UAS responder line with a BCD isoform, a sensor line carrying a cad 3'UTR homologue and nos-GAL4:VP16. Represented in each table are the values of each experiment and the respective control, which was used for normalization (relative average value set to 1 )

\begin{tabular}{|l|l|l|l|l|l|l|}
\hline maternal genotype & $\mathrm{n}$ & average & se & $\begin{array}{l}\text { Relative } \\
\text { average }\end{array}$ & Relative se & $p$ (ttest) \\
\hline $\begin{array}{l}\text { 38F-BRE_257-319/+;nos- } \\
\text { GAL4:VP16/+ }\end{array}$ & 20 & 25.62205 & 0.737960516 & 1 & 0.028801775 & \\
\hline $\begin{array}{l}\text { UAS-BcdG } \\
\text { BRE_M6/+;38F- } \\
\text { GAL4:VP16/+ }\end{array}$ & 20 & 15.5258 & 0.63337806 & 0.605954637 & 0.024720038 & $\begin{array}{l}1.56169 \mathrm{E}- \\
12\end{array}$ \\
\hline
\end{tabular}

\begin{tabular}{|l|l|l|l|l|l|l|}
\hline maternal genotype & $\mathrm{n}$ & average & se & $\begin{array}{l}\text { Relative } \\
\text { average }\end{array}$ & Relative se & $p$ (ttest) \\
\hline $\begin{array}{l}\text { 38F-SV40/+;nos- } \\
\text { GAL4:VP16/+ }\end{array}$ & 23 & 43.20817391 & 1.950060582 & 1 & 0.045131752 & \\
\hline $\begin{array}{l}\text { UAS- } \\
\text { BcdG } \\
\text { SV4M6/+;/;38F- } \\
\text { GAL4:VP16/+ }\end{array}$ & 25 & 40.22504 & 1.6483047 & 0.930959038 & 0.038147983 & 0.248962738 \\
\hline
\end{tabular}

\begin{tabular}{|l|l|l|l|}
\hline maternal genotype & Relative average & Relative se & $p$ (ttest) \\
\hline 38F-BRE_257-319/+;nos-GAL4:VP16/+ & 0.592990809 & 0.017079188 & \\
\hline 38F-SV40/+;nos-GAL4:VP16/+ & 1 & 0.045131752 & $3.49225 E-09$ \\
\hline
\end{tabular}

\begin{tabular}{|c|c|c|c|c|c|c|}
\hline maternal genotype & $\mathrm{n}$ & average & se & $\begin{array}{l}\text { Relative } \\
\text { average }\end{array}$ & Relative se & $p$ (ttest) \\
\hline $\begin{array}{l}\text { 38F-H1/+;nos- } \\
\text { GAL4:VP16/+ }\end{array}$ & 21 & 102.3991429 & 3.528145895 & 1 & 0.034454838 & \\
\hline $\begin{array}{l}\text { UAS- } \\
\text { BcdG }^{\text {F4M6 } /+; 38 F-} \\
\text { H1/+;nos- } \\
\text { GAL4:VP16/+ }\end{array}$ & 22 & 105.3981364 & 3.779641716 & 1.029287291 & 0.036910873 & 0.565077385 \\
\hline
\end{tabular}




\begin{tabular}{|l|l|l|l|l|l|l|}
\hline maternal genotype & $\mathrm{n}$ & average & se & $\begin{array}{l}\text { Relative } \\
\text { average }\end{array}$ & Relative se & $p$ (ttest) \\
\hline $\begin{array}{l}\text { 38F-H2/+;nos- } \\
\text { GAL4:VP16/+ }\end{array}$ & 54 & 19.33198148 & 0.513318512 & 1 & 0.026552814 & \\
\hline $\begin{array}{l}\text { UAS- } \\
\text { BcdGF4M6/+;38F- } \\
\text { H2/+;nos- } \\
\text { GAL4:VP16/+ }\end{array}$ & 48 & 16.83685417 & 0.571623338 & 0.870932665 & 0.029568792 & 0.001537634 \\
\hline
\end{tabular}

\begin{tabular}{|l|l|l|l|l|l|l|}
\hline maternal genotype & $\mathrm{n}$ & average & se & $\begin{array}{l}\text { Relative } \\
\text { average }\end{array}$ & Relative se & $p$ (ttest) \\
\hline $\begin{array}{l}\text { 38F-H3/+;nos- } \\
\text { GAL4:VP16/+ }\end{array}$ & 20 & 20.02185 & 0.922385335 & 1 & 0.046068936 & \\
\hline $\begin{array}{l}\text { UAS- } \\
\text { BcdG FM6/+;38F- } \\
\text { H3/+;nos- } \\
\text { GAL4:VP16/+ }\end{array}$ & 26 & 18.99507692 & 0.723879499 & 0.948717372 & 0.036154476 & 0.386622815 \\
\hline
\end{tabular}

\begin{tabular}{|l|l|l|l|l|l|l|}
\hline maternal genotype & $\mathrm{n}$ & average & se & $\begin{array}{l}\text { Relative } \\
\text { average }\end{array}$ & Relative se & $p$ (ttest) \\
\hline $\begin{array}{l}\text { 38F-Hp3'/+;nos- } \\
\text { GAL4:VP16/+ }\end{array}$ & 19 & 81.37915789 & 2.02269139 & 1 & 0.024855153 & \\
\hline $\begin{array}{l}\text { UAS-BcdG } \\
\text { Hp3'/+;/+;8F- } \\
\text { GAL4:VP16/+ }\end{array}$ & 23 & 65.83321739 & 1.99342019 & 0.808969017 & 0.024495463 & $\begin{array}{l}2.97204 \mathrm{E}- \\
06\end{array}$ \\
\hline
\end{tabular}

\begin{tabular}{|l|l|l|l|l|l|l|}
\hline maternal genotype & $\mathrm{n}$ & average & se & $\begin{array}{l}\text { Relative } \\
\text { average }\end{array}$ & Relative se & $p$ (ttest) \\
\hline $\begin{array}{l}\text { 38F-H2/+;nos- } \\
\text { GAL4:VP16/+ }\end{array}$ & 34 & 74.20338235 & 1.573567109 & 1 & 0.021206137 & \\
\hline $\begin{array}{l}\text { 38F-H2/+;UAS- } \\
\text { BcdF } \\
\text { GAL4:VPos- }\end{array}$ & 44 & 69.73513636 & 1.390376016 & 0.939783796 & 0.018737367 & 0.036802706 \\
\hline
\end{tabular}

\begin{tabular}{|l|l|l|l|l|l|l|}
\hline maternal genotype & $\mathrm{n}$ & average & se & $\begin{array}{l}\text { Relative } \\
\text { average }\end{array}$ & Relative se & $p$ (ttest) \\
\hline $\begin{array}{l}\text { 38F-Tc3'/+;nos- } \\
\text { GAL4:VP16/+ }\end{array}$ & 20 & 35.2917 & 1.64440782 & 1 & 0.046594747 & \\
\hline $\begin{array}{l}\text { UAS-BcdG } \\
\text { Tc3/1/+;/nos- } \\
\text { GAL4:VP16/+ }\end{array}$ & 20 & 32.6276 & 1.729211975 & 0.924511996 & 0.048997696 & 0.271264825 \\
\hline
\end{tabular}


UAS BCD isoforms + 38F-Dm3'mut: Measurements taken from photographs of embryos deriving from females expressing UAS responder line with a BCD isoform, sensor line 38F-Dm3'mut and nos-GAL4:VP16. Represented in each table are the values of each experiment and the respective control, which was used for normalization (relative average value set to 1 ).

\begin{tabular}{|l|l|l|l|l|l|l|}
\hline maternal genotype & $\mathrm{n}$ & average & se & $\begin{array}{l}\text { Relative } \\
\text { average }\end{array}$ & Relative se & $p$ (ttest) \\
\hline $\begin{array}{l}\text { 38F-Dm3'/+;nos- } \\
\text { GAL4:VP16/+ }\end{array}$ & 27 & 23.09955556 & 0.965158605 & 1 & 0.041782562 & \\
\hline $\begin{array}{l}\text { 38F-Dm3'mut/+;nos- } \\
\text { GAL4:VP16/+ }\end{array}$ & 24 & 43.42158333 & 1.604758527 & 1.879758388 & 0.069471403 & $\begin{array}{l}3.1084 \mathrm{E}- \\
13\end{array}$ \\
\hline $\begin{array}{l}\text { UAS-BCDG } \\
\text { Pm3'/m6/+38F- } \\
\text { GAL4:VP16/+ }\end{array}$ & 31 & 41.59634483 & 1.764890024 & 1.800742214 & 0.076403636 & 0.4560315 \\
\hline
\end{tabular}

\begin{tabular}{|l|l|l|l|l|l|l|}
\hline maternal genotype & $\mathrm{n}$ & average & se & $\begin{array}{l}\text { Relative } \\
\text { average }\end{array}$ & Relative se & $p$ (ttest) \\
\hline $\begin{array}{l}\text { Dm3'mut/+;nos- } \\
\text { GAL4:VP16/+ }\end{array}$ & 33 & 45.52769697 & 1.252315518 & 1 & 0.027506674 & \\
\hline $\begin{array}{l}\text { Dm3'mut/+;UAS- } \\
\text { BcdD } \\
\text { GAL4:VP16 }\end{array}$ & 31 & 43.64764516 & 0.946339261 & 0.958705317 & 0.020786012 & 0.235840665 \\
\hline $\begin{array}{l}\text { Dm3'mut/+;UAS- } \\
\text { BcdD } \\
\text { GAL4:/nos- }\end{array}$ & 32 & 46.87709375 & 1.002291348 & 1.02963903 & 0.02201498 & 0.403515702 \\
\hline $\begin{array}{l}\text { Dm3'mut/+;UAS- } \\
\text { BcdF } \\
\text { GAL4:VP16 }\end{array}$ & 8 & 46.4595 & 1.945114273 & 1.020466729 & 0.042723757 & 0.69341138 \\
\hline
\end{tabular}

\begin{tabular}{|l|l|l|l|l|l|l|}
\hline maternal genotype & $\mathrm{n}$ & average & se & $\begin{array}{l}\text { Relative } \\
\text { average }\end{array}$ & Relative se & $p$ (ttest) \\
\hline $\begin{array}{l}\text { 38F-Dm3'mut/+;nos- } \\
\text { GAL4:VP16/+ (II) }\end{array}$ & 34 & 47.95935294 & 1.038642895 & 1 & 0.021656733 & \\
\hline $\begin{array}{l}\text { 38F- } \\
\text { Dm3'mut/+;UAS- } \\
\text { BcdEF'/nos- } \\
\text { GAL4:VP16 }\end{array}$ & 37 & 50.5202973 & 0.853551302 & 1.053398226 & 0.01779739 & 0.061192215 \\
\hline
\end{tabular}

\begin{tabular}{|l|l|l|l|l|l|l|}
\hline maternal genotype & $\mathrm{n}$ & average & se & $\begin{array}{l}\text { Relative } \\
\text { average }\end{array}$ & Relative se & $p$ (ttest) \\
\hline $\begin{array}{l}\text { 38F-Dm3'mut/+;nos- } \\
\text { GAL4:VP16/+ }\end{array}$ & 32 & 48.2561875 & 1.011951014 & 1 & 0.020970389 & \\
\hline $\begin{array}{l}\text { 38F- } \\
\text { Dm3'mut/+;UAS- } \\
\text { BcdF } \\
\text { GAL4:/nos- } \\
\text { GAP16 }\end{array}$ & 22 & 51.35718182 & 1.119409746 & 1.064261072 & 0.023197227 & 0.045374255 \\
\hline
\end{tabular}




\section{Curriculum vitae}

\section{Personal details}

Name:

Claudia Jasmin Rödel

Date of birth:

25.04.1981

Place of birth:

Bremerhaven, Germany

\section{Address of current working address}

Institute for Molecular Biology and Biotechnology - FoRTH

Nikolaou Plastira 100

GR-70013, Heraklion, Crete

Greece

Telephone: +302810391148

Email: roedel@imbb.forth.gr

\section{University studies}

University:

Country:

Subjects:

From:

To:

Degree:

Thesis:

Supervisor of thesis:

University:

Country:

From:

To:

Purpose:
Georg-August-University Göttingen, Germany

Germany

Developmental biology, zoology and biochemistry

October 2000

June 2006

Diploma

"Isolation of developmental genes from the indian stick insect Carausius morosus" at the Dept. of Developmental Biology, GZMB, Georg-August-University Göttingen

Prof. Dr. Ernst A. Wimmer

University of Salamanca, Spain

Spain

October 2004

March 2005

Exchange semester supported by the ERASMUS/SOCRATES

Exchange Program

\section{PhD thesis}

University:

Country:

Georg-August-University Göttingen, Germany

From:

Thesis:

Greece

July 2006

"Evolution of caudal translational repression in higher insects", at the Dept. of Developmental Biology, Institute of Molecular Biology and Biotechnology - FoRTH,

GR-70013 Heraklion, Greece

External supervisor: Dr. Michalis Averof

Supervisor of thesis: Prof. Dr. Ernst A. Wimmer 
Funding:

July 2006 - June 2009, Scholarship awarded by the Marie Curie Early Stage Training Program "Functional analysis of microRNAs during early development",

October 2009 - June 2010, Scholarship of the Greek Government to foreign citizens awarded by the Hellenic Republic, Ministry of Education, Lifelong Learning and Religious Affairs.

October 2010 - June 2011, Scholarship of the Greek Government to foreign citizens awarded by the Hellenic Republic, Ministry of Education, Lifelong Learning and Religious Affairs.

\section{Experiences}

Student assistant: $\quad$ April 2003 - September 2003, April 2004 - September 2004, Zoological anatomical practical course for beginners.

April 2005 - September 2005, Animal identification.

October 2005 - March 2006, Practical course and tutorial of practical course of biology for medical students.

Johann-Friedrich-Blumenbach-Institute of Zoology and Anthropology, Georg-August-University Göttingen, Germany 


\section{Acknowledgements}

I want to thank Dr. Michalis Averof for his scientific guidance and support during my $\mathrm{PhD}$ and for being available for discussions at all times. I am very grateful for his supervision, for allowing me to be part of his research group and for creating an inspiring scientific enviroment. Furthermore I would like to thank him for his support in all other matters outside the lab.

I want to thank my referees Prof. Ernst Wimmer and Prof. Gregor Bucher for their support and help and for the fruitful comments and constructive discussions. I want to thank Prof. Wimmer for having it made possible for me to conduct my research in Crete.

I would like to thank Prof. Andre Fiala, Prof. Reinhard Schuh, Prof. Andreas Stumpner and Prof. Andreas Wodarz, who agreed to be members of my commission board.

I would like to thank all members of the Averof Lab and the Delidakis Lab at the IMBB, Crete for their immediate hospitality, kindness, and friendliness. They created a pleasant and genial working environment during lab meetings, round tables, journal clubs and in the our joint lab. In particular I want to thank Alexandros Kioupakis for his invaluable help during the early stages of my PhD, he has become a big hero of mine.

I want to thank Giannis Livadaras for performing the injections in Drosophila embryos for the transgenesis experiments.

I would like to thank Prof. Anastassios Economou for providing the $\mathrm{pET16b-HisMBP}$ plasmid and Prof. Urs Schmidt-Ott for providing the cad 3'UTR of Haematopota pluvialis.

I would like to thank my friends, who have become family in Crete, making this island what it is to me, a place that you never want to leave.

I want to thank my family in Germany for their short and long distance support, understanding and love during my $\mathrm{PhD}$ and all stages of my life.

Especially I want to thank my (to be) husband George, who is the only one who really understands me, for his love and support. 